\title{
Computational Fluid Dynamic Studies of Chemical Looping Combustion Systems
}

Kartikeya Krishnaji Mahalatkar

West Virginia University

Follow this and additional works at: https://researchrepository.wvu.edu/etd

\section{Recommended Citation}

Mahalatkar, Kartikeya Krishnaji, "Computational Fluid Dynamic Studies of Chemical Looping Combustion Systems" (2010). Graduate Theses, Dissertations, and Problem Reports. 3008.

https://researchrepository.wvu.edu/etd/3008

This Dissertation is protected by copyright and/or related rights. It has been brought to you by the The Research Repository @ WVU with permission from the rights-holder(s). You are free to use this Dissertation in any way that is permitted by the copyright and related rights legislation that applies to your use. For other uses you must obtain permission from the rights-holder(s) directly, unless additional rights are indicated by a Creative Commons license in the record and/ or on the work itself. This Dissertation has been accepted for inclusion in WVU Graduate Theses, Dissertations, and Problem Reports collection by an authorized administrator of The Research Repository @ WVU.

For more information, please contact researchrepository@mail.wvu.edu. 


\title{
Computational Fluid Dynamic Studies of Chemical Looping Combustion Systems
}

\author{
Submitted by \\ Kartikeya Krishnaji Mahalatkar \\ Dissertation \\ submitted to the \\ College of Engineering and Mineral Resources \\ at West Virginia University \\ in partial fulfillment of the requirements \\ for the degree of

\section{Doctor \\ of \\ Philosophy}

\section{Committee Members:}

Prof. John Kuhlman, Chair

Prof. Ismail Celik

Prof. Eric Johnson

Dr. Thomas O'Brien, NETL/DOE

Prof. Richard Turton

\section{Department of Mechanical and Aerospace Engineering \\ Morgantown, West Virginia \\ 2010}

Keywords: CFD, Combustion, Chemical looping, Heterogeneous reactions, Multiphase flow. 


\title{
$\underline{\text { Abstract }}$ \\ Computational Fluid Dynamic Studies of Chemical Looping Combustion Systems \\ Kartikeya Krishnaji Mahalatkar
}

\begin{abstract}
Responsible carbon management will be required for the future utilization of fossil fuels for power generation. One technology that is showing tremendous potential for carbon capture is the chemical looping combustion (CLC). CLC involves combustion of fuels by heterogeneous chemical reactions with an oxygen carrier, usually a granular metal oxide, exchanged between two fluidized beds. In any regular combustion process the oxidizer for the fuel is air which essentially is a mixture of oxygen and nitrogen. This results in a flue gas stream which consists of primarily of relatively dilute $\mathrm{CO}_{2}(15-$ $20 \%$ ), mixed with steam and nitrogen. In $\mathrm{CLC}, \mathrm{CO}_{2}$ separation is easily achieved because the oxygen for the reaction is supplied by the carrier (e.g. a metal oxide), resulting in a flue gas stream consisting almost entirely of carbon dioxide and steam. The steam can be easily condensed to give pure $\mathrm{CO}_{2}$ which can then be easily sequestrated. Thus the CLC process for power generation provides a sequestration ready $\mathrm{CO}_{2}$ stream without the need for using costly gas separation techniques. The only operational penalty for CLC is then the slight pressure losses required to circulate the carrier between reactors and the carrier make-up costs. CLC requires many unit operations involving gas-solid or granular flow. Herein a computational fluid dynamic study is presented to analyze the performance of CLC systems utilizing both gaseous and solid fuels. There have been extensive experimental studies in CLC, however CFD simulations of this concept to date are quite limited. The present simulations were performed using the interpenetrating fluid representation of dense multiphase flow. The ANSYS-FLUENT ${ }^{\mathrm{TM}}$ CFD solver was used in the present study. The granular phases are represented as continua whose dynamics are governed by Navier-Stokes like equations, coupled to the N-S equations describing the fluid flow. Detailed sub-models to account for fluid-particle and particle-particle interaction forces have been included. Heat transfer is fully accounted for. Heterogeneous reactions are used to describe the coal conversion and the reactions of the gaseous fuel with the carrier. Global chemical reaction models of fuel and carrier were utilized. Capability of the model to simulate the segregation processes, depending on particle density and size differences between the carrier and the fuel, allows the design of a reactor with the desired behavior. The results obtained from CFD have been compared with available experimental information. The transient CFD simulations provided a reasonable agreement with the reported experimental data for batch reactors using gaseous as well as solid fuels, and for a full circulating fluid bed CLC using gaseous fuels.
\end{abstract}


The CFD models that were developed and validated using available experimental results have been applied for design evaluations of fuel reactor of CLC system utilizing char as fuel. It would be very desirable to utilize coal directly in the fuel reactor, which requires in situ gasification in either a moving or bubbling fluidized bed reactor. In such a design, $\mathrm{H}_{2} \mathrm{O}\left(\right.$ and $\mathrm{CO}_{2}$ ) must be recycled from the product stream as the fluidizing medium, which allows in-bed heat transfer and mediates chemical reactions between the two solid feeds (carrier and fuel), and gasifies the coal char. The solid coal fuel must be heated by the recycled metal oxide, driving off moisture and volatile material, and the remaining char must then be gasified to provide complete fuel utilization. The gaseous products of these reactions must then contact the hot, oxidized carrier before leaving the bed to obtain complete conversion of the fuel to $\mathrm{H}_{2} \mathrm{O}$ and $\mathrm{CO}_{2}$. Further, the reduced carrier particles must be removed from the bed and returned as a pure stream to the air reactor for regeneration. It is critical that no unburned fuel, i.e., char, be returned with the spent carrier as this material will rapidly burn in the air reactor and the resulting $\mathrm{CO}_{2}$ will escape capture. Three designs have been developed and analyzed with CFD. Special attention is paid to Fe-based carriers (due to their low cost relative to other carriers), which is somewhat complicated due to the multiple oxidation states displayed by Fe. The non-linear interaction of factors such as multiphase hydrodynamics, heat transfer and chemical reaction is fully coupled in the numerical simulations allowing evaluation of design options for a full circulating CLC system using solid fuels. 


\section{Table of Contents}

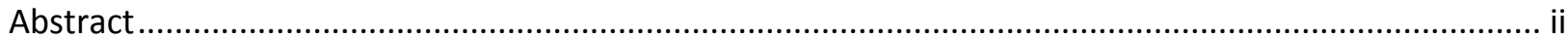

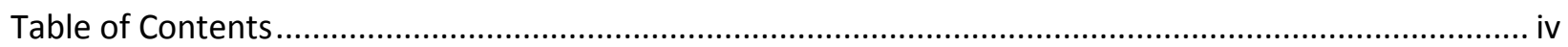

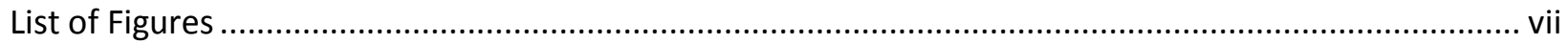

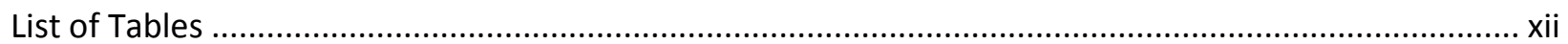

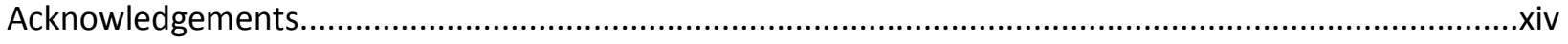

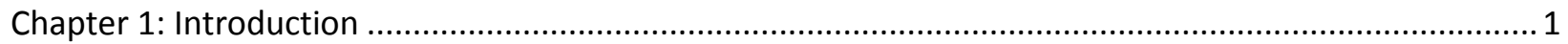

Chapter 2: Carbon Capture and Storage Technologies........................................................................ 4

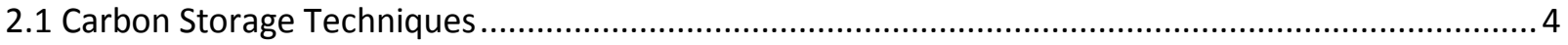

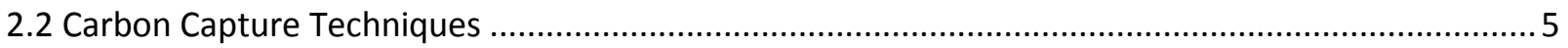

2.3: Cost comparisons for various carbon capture technologies .................................................. 6

Chapter 3: Thermodynamic Cycle Analysis for Design of CLC ............................................................. 8

Chapter 4: CFD Flow Model for Granular Multiphase Flows .............................................................. 22

Chapter 5: Basic Validation Studies for Granular Flows ....................................................................... 33

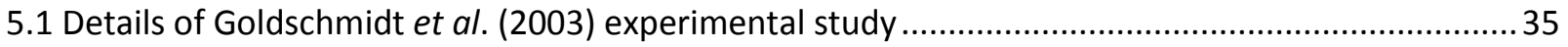

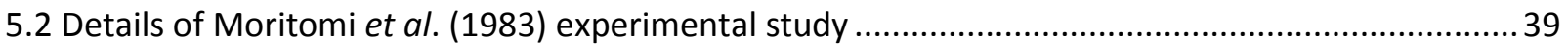

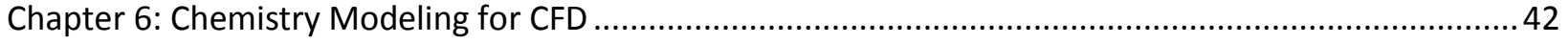

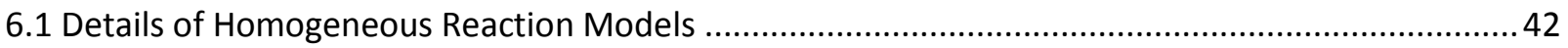

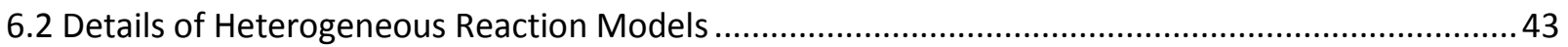

Chapter 7: Simulations of Batch Reactor for Gaseous Fuels ................................................................ 53

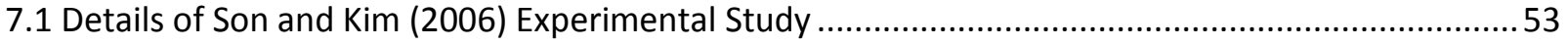

7.2 Details of Mattisson et al. (2001) experiments ............................................................................ 54

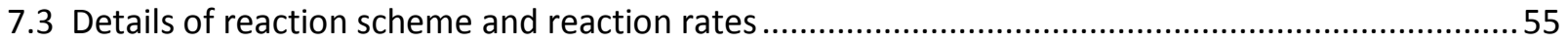

7.4 Details of Numerics for Son and Kim (2006) experiments and Grid Convergence Study:.................61

7.5 Details of Numerics for Mattisson et al. (2001) experiments ......................................................64

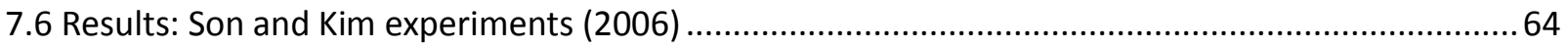

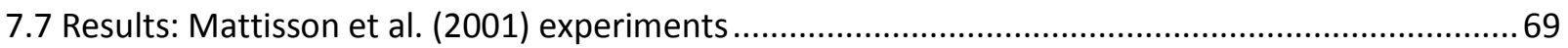
iv 
7.8 Conclusion

Chapter 8: Simulations of Batch Reactor for Coal Combustion ............................................................ 77

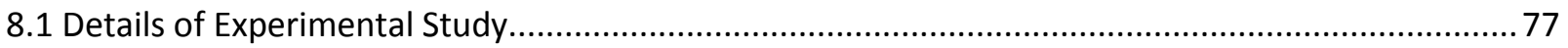

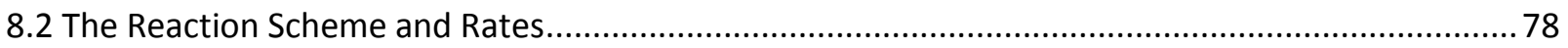

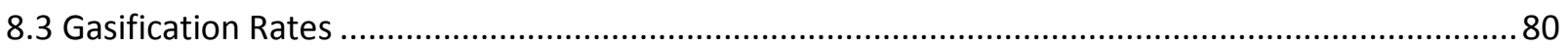

8.4 Water-Gas-Shift Reaction Rates (Bustamante et al., 2004) ............................................................ 82

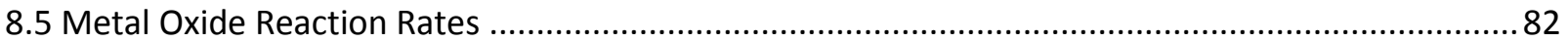

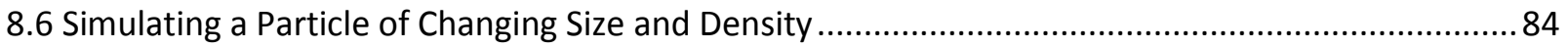

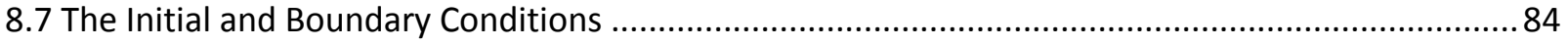

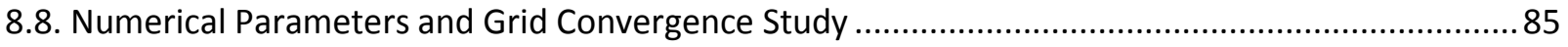

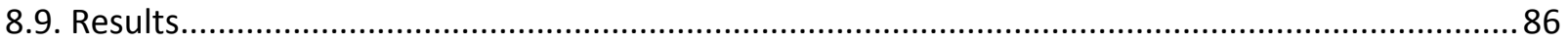

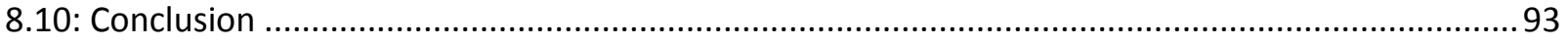

Chapter 9: Simulations of Full Circulating Fluidized Bed CLC Systems …............................................. 94

9.1 Details of Kronberger et al. (2004) experimental study ............................................................96

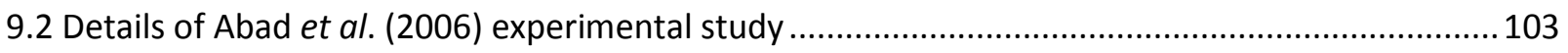

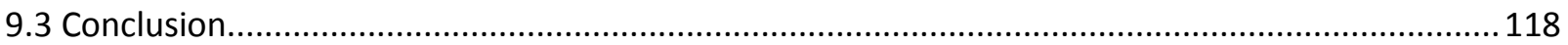

Chapter 10: Design of Fuel Reactor utilizing Solid Fuel (Char) ......................................................... 119

10.1: Performance of existing CLC systems utilizing solid fuels ...................................................... 120

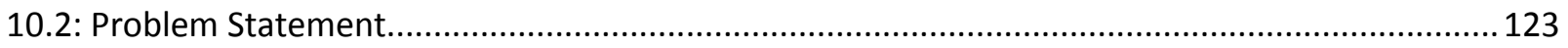

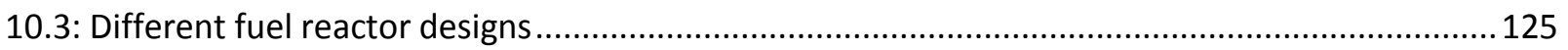

10.4: Comparison of Designs 1, 2 and 3 and other experimental studies:..................................... 146

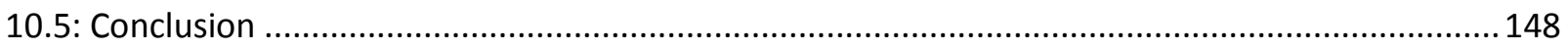

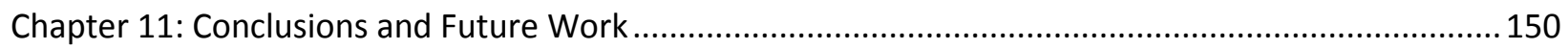

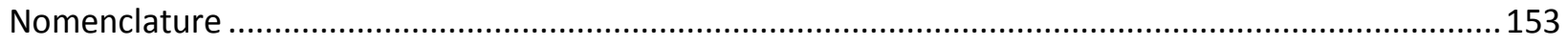

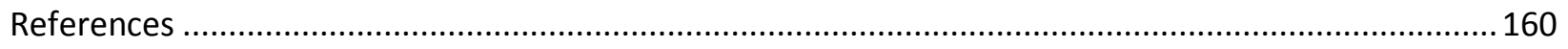


APPENDIX 1: Detailed calculations for volatile composition and gasification products for South African

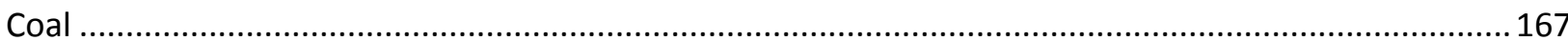

APPENDIX 2: User Defined Functions for Coal Combustion Studies..................................................... 172

APPENDIX 3: User Defined Functions for Gaseous Fuel Combustion ....................................................... 182

APPENDIX 4: User Defined Functions for Langroundi et al. Viscoplastic Model .................................... 185

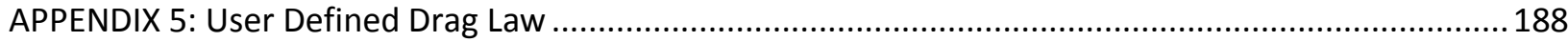




\section{List of Figures}

Fig. 1.1: Schematic of Chemical Looping Combustion (CLC) system (Lyngfelt et al., 2001) ....................... 2

Fig. 2.1: Efficiency penalties for various carbon capture technologies. .............................................. 6

Fig. 2.2: Cost penalties for various carbon capture technologies........................................................ 7

Fig. 3. 1: Control volumes used for energy balance of AR and FR reactors.......................................... 13

Fig. 3. 2: Flow chart for the iterative procedure for calculating CLC system mass and energy balance. ...19

Fig. 4.1: Location of Drucker-Prager (1952) yield surface on two-dimensional principle stress plane...... 29

Fig. 4.2: Location of Drucker-Prager (1952) yield surface on three-dimensional principle stress space. ...29

Fig. 4.3: Location of yield surface for Pitman-Schaeffer (1987) model on two-dimensional principle stress plane.

Fig. 4.4: Location of yield surface for Pitman-Schaeffer (1987) model on three-dimensional principle stress space.

Fig. 5.1: Distance from distributor vs. average bubble diameter (Chandrasekaran et al., 2005). .34

Fig. 5.2: Average bubble diameter vs. average vertical bubble velocity (Chandrasekaran et al., 2005).... 34

Fig. 5.3: Average bed heights for small and large particles with time for simulations of Goldschmidt et al. (2003) experiments.

Fig. 5.4: Particle volume fraction for large and small particles at time $=30$ seconds $(25 \%$ small particles, superficial velocity $=1.1 \mathrm{~m} / \mathrm{s}$, bed height $=15 \mathrm{~cm}$ ).

Fig. 5.5: Variation in experimental and predicted segregation extent with time (superficial velocity = $1.1 \mathrm{~m} / \mathrm{s}$ and bed height $=15 \mathrm{~cm}$ ) for various concentrations of small particles. 38

Fig. 5.6: Variation in experimental and predicted segregation extent with time (superficial velocity = $1.1 \mathrm{~m} / \mathrm{s}$ and $50 \%$ small particles) for two different bed heights.

Fig. 5.7: Variation in experimental and predicted segregation extent with time (Bed height $=15 \mathrm{~cm}$ and $50 \%$ small particles) for two different fluidization velocity.

Fig. 5. 8: Comparison of experimental and predicted Interface location of glass beads and hollow char. 41

Fig. 6. 1: Schematic representation of the Shrinking Core Model for non-porous particles (Szekely et al., 1976). 44

vii 
Fig. 6. 2: Schematic representation of a porous, spherical particle with spherical grains (Szekely et al., 1976). Dark grains indicate the unreacted core and lighter colored grains indicate reacted outer core. . 49

Fig. 7. 1: Full CLC system studied by Son and Kim (2006)...... 61

Fig. 7. 2: Fine vs. Coarse grid (superficial velocity of $50 \mathrm{~mm} / \mathrm{sec}$ and operating temperature of $1123 \mathrm{~K}$ )...63

Fig. 7. 3: Time variation of $\mathrm{CH}_{4}$ concentration (superficial velocity of $25 \mathrm{~mm} / \mathrm{sec}$ and operating temperature of $1123 \mathrm{~K})$. .66

Fig. 7. 4: Outlet composition of flue gases at various $\mathrm{Fe}_{2} \mathrm{O}_{3}$ concentration for Son and Kim experiments (2006), (100\% indicates only $\mathrm{Fe}_{2} \mathrm{O}_{3}$ is present and $0 \%$ indicates only $\mathrm{NiO}$ is present).

Fig. 7. 5: Contour Plots for Son and Kim (2006) experiments [NiO-100\%, velocity $=50 \mathrm{~mm} / \mathrm{s}$.

Fig. 7. 6: Gas phase volume fraction contour for Son and Kim (2006) experiments [NiO-75\%, Temperature $=1123 \mathrm{~K}]$.

Fig. 7. 7: Contour and Vector Plots for Son and Kim (2006) experiments [NiO-75\%, velocity $=25 \mathrm{~mm} / \mathrm{s}$, Temperature $=1123 \mathrm{~K}]$. Computational domain is truncated to show the bubble regions and gas velocity vectors.

Fig. 7. 8: Outlet composition of flue gases at various velocities for Son and Kim (2006) experiments (NiO:Fe $\left.\mathrm{O}_{3}:: 75: 25\right) \ldots$

Fig. 7. 9: Variation in Concentration with Temperature.

Fig. 7. 10: Outlet Flow concentration from simulations ignoring the formation of wustite or FeO (Mattisson et al. experiments, 2001).

Fig. 7. 11: Time varying mass of hematite $\left(\mathrm{Fe}_{2} \mathrm{O}_{3}\right)$, magnetite $\left(\mathrm{Fe}_{3} \mathrm{O}_{4}\right)$ and wustite $(\mathrm{FeO})$ in the bed (Mattisson et al. experiments, 2001).

Fig. 7. 12: Outlet flow concentration from simulations including the formation of wustite (Experiments of Mattisson et al., 2001).

Fig. 8. 1: The lower portion of the computational mesh. The mesh is mirrored about the axis. .85

Fig. 8. 2: Comparison of the calculated time development of the $\mathrm{CO}_{2}$ and $\mathrm{CO}$ concentrations using a coarse and a fine mesh (Temperature of $950{ }^{\circ} \mathrm{C}$ and $\mathrm{H}_{2} \mathrm{O}$ concentration of $50 \%$ ).

Fig. 8. 3: Velocity vectors $(\mathrm{m} / \mathrm{s})$ and volume fractions of iron oxide and coal particles at 175 seconds, temperature of $1273 \mathrm{~K}$ and $50 \%$ steam inlet concentration.

Fig. 8. 4: Instantaneous gas velocity vectors (m/s) [Fig. 5a] and gas species mass fractions at 175 seconds, temperature of $1273 \mathrm{~K}$ and $50 \%$ steam inlet concentration.

viii 
Fig. 8.5: Calculated concentrations of $\mathrm{CO}_{2}, \mathrm{CO}$ and $\mathrm{CH}_{4}$ at a height of $3 \mathrm{~m}$ compared to the experimental data of Leion et al., 2008. The temperature is $1223 \mathrm{~K}$ and $50 \%$ inlet steam concentration is used. 90

Fig. 8. 6: Local gasification rates of char particles $\left(\mathrm{kmol} / \mathrm{m}^{3} / \mathrm{s}\right)$ at $175 \mathrm{~seconds}$, temperature of $1273 \mathrm{~K}$ and $50 \%$ steam inlet concentration.

Fig. 8. 7: Time variation of hematite $\left(\mathrm{Fe}_{2} \mathrm{O}_{3}\right)$ and magnetite $\left(\mathrm{Fe}_{3} \mathrm{O}_{4}\right)$ mass. Temperature of $1223 \mathrm{~K}$ and $50 \%$ steam concentration.

Fig. 8. 8: The average coal reaction rate (see text) at different operating temperatures (50\% Steam concentration).

Fig. 8. 9: The average coal reaction rate (see text) at different steam concentration, temperature of $1223 \mathrm{~K}$.

Fig. 9. 1: Full CLC system studied by Kronberger et al. (2004).

Fig. 9. 2: Mesh used for simulation of Kronberger experiments (2004).

Fig. 9. 3: Comparison of solid circulation rates predicted by Schaeffer model (1987) and that of Langroudi et al. (2010). Slot height for the experiment was $1.5 \mathrm{~mm}, \mathrm{U}_{\mathrm{FR}}=18 \mathrm{U}_{\mathrm{mf}}$ and $\mathrm{U}_{\mathrm{AR}}=1.8 \mathrm{U}_{\mathrm{t}}$. 100

Fig. 9. 4: Instantaneous contour plots at 6 seconds $\left(U_{F R}=18 U_{m f}\right.$ and $\left.U_{A R}=1.8 U_{t}\right)$. 101

Fig. 9. 5: Comparison of solid circulation rates with experiments. Two values of transition volume fraction $\alpha_{s, \text { min }}^{*}$ have been used $(0.5$ and 0.53$)$ to check the sensitivity of this parameter $\left(\mathrm{U}_{\mathrm{FR}}=18 \mathrm{U}_{\mathrm{mf}}\right)$.

Fig. 9. 6: Gas leakage into Air Reactor. Two values of transition volume fraction $\alpha^{*}{ }_{s \text {,min }}$ have been used $(0.5$ and 0.53$)$ to check the sensitivity of this parameter $\left(U_{F R}=18 U_{m f}\right)$. 102

Fig. 9. 7: Gas leakage into Fuel Reactor. Two values of transition volume fraction $\alpha^{*}{ }_{s \text {,min }}$ have been used ( 0.5 and 0.53$)$ to check the sensitivity of this parameter $\left(U_{\mathrm{FR}}=18 \mathrm{U}_{\mathrm{mf}}\right)$. 103

Fig. 9. 8: Fine mesh used in the numerical study. 105

Fig. 9. 9: Gas volume fraction and species mole fraction contour of various gases (Operating temperature of $1123 \mathrm{~K})$. FR flow rate of $7.5 \times 10^{-6} \mathrm{~m}^{3} / \mathrm{s}$ and AR flow rate of $83 \times 10^{-6} \mathrm{~m}^{3} / \mathrm{s}$.

Fig. 9. 10: Variation in outlet $\mathrm{CH}_{4}$ fraction $\left(\mathrm{X}_{\mathrm{CH}_{4}} /\left(\mathrm{X}_{\mathrm{CH}_{4}}+\mathrm{X}_{\mathrm{CO}}+\mathrm{X}_{\mathrm{CO} 2}\right)\right)$, with change in $\mathrm{FR}$ flow rates (FR flow rates varies from $2.5 \times 10^{-6}$ to $7.5 \times 10^{-6} \mathrm{~m}^{3} / \mathrm{s}$ and $A R$ flow rates is constant at $83 \times 10^{-6} \mathrm{~m}^{3} / \mathrm{s}$ ).

Fig. 9. 11: Variation in outlet $\mathrm{CH}_{4}$ fraction $\left(\mathrm{X}_{\mathrm{CH} 4} /\left(\mathrm{X}_{\mathrm{CH} 4}+\mathrm{X}_{\mathrm{CO}}+\mathrm{X}_{\mathrm{CO} 2}\right)\right)$, with change in operating temperature of oven (Temperature changes from $1073 \mathrm{~K}$ to $1223 \mathrm{~K}$ ) and change in AR flow rates (AR flow rates varies from $66.7 \times 10^{-6}$ to $91.7 \times 10^{-6} \mathrm{~m}^{3} / \mathrm{s}$ ). 
Fig. 9. 12: Variation in solid circulation rate $\left(\mathrm{kg} / \mathrm{s} \times 10^{3}\right)$ with change in fuel reactor flow rates (FR flow rates varies from $2.5 \times 10^{-6}$ to $7.5 \times 10^{-6} \mathrm{~m}^{3} / \mathrm{s}$ ). AR $=83 \times 10^{-6} \mathrm{~m}^{3} / \mathrm{s}$

Fig. 9. 13: Variation in solid circulation rate $\left(\mathrm{kg} / \mathrm{s} \times 10^{3}\right)$ with change in air reactor flow rates (AR flow rates vary from $66.7 \times 10^{-6}$ to $\left.91.7 \times 10^{-6} \mathrm{~m}^{3} / \mathrm{s}\right) . \mathrm{FR}=5.8 \times 10^{-6} \mathrm{~m}^{3} / \mathrm{s}$. 115

Fig. 9. 14: Predicted gas flow rates $\left(\mathrm{kg} / \mathrm{s} \times 10^{6}\right)$ through the slot and downcomer. Air reactor flow rate is $83.0 \times 10^{-6} \mathrm{~m}^{3} / \mathrm{s}$. FR flow rate is $7.5 \times 10^{-6} \mathrm{~m} 3 / \mathrm{s}$. Positive mass flow rates indicate flow from FR to AR. 116

Fig. 9. 15: Variation in leakage and dilution (\% Vol) with change in fuel reactor flow rates (FR flow rates vary from $2.5 \times 10^{-6}$ to $7.5 \times 10^{-6} \mathrm{~m}^{3} / \mathrm{s}$ ). AR flow rate was $83 \times 10^{-6} \mathrm{~m}^{3} / \mathrm{s}$. 116

Fig. 9. 16: Variation in leakage and dilution (\%Vol) with change in air reactor flow rates (AR flow rates vary from $66.7 \times 10^{-6}$ to $91.7 \times 10^{-6} \mathrm{~m}^{3} / \mathrm{s}$ ). FR flow rate was $5.8 \times 10^{-6} \mathrm{~m}^{3} / \mathrm{s}$.

Fig. 9. 17: Variation in carbon capture efficiency (\%) with change in fuel reactor and air reactor flow rates (FR flow rate varies from $2.5 \times 10^{-6}$ to $7.5 \times 10^{-6} \mathrm{~m}^{3} / \mathrm{s}$ and AR flow rates varies from $66.7 \times 10^{-6}$ to $91.7 \mathrm{x}$

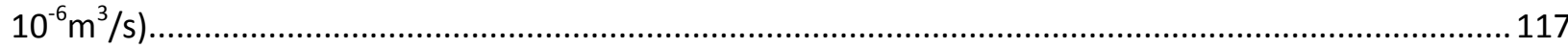

Fig. 10. 1: Design 1 for the FR utilizing char as fuel.

Fig. 10. 2: Instantaneous volume fraction, $20 \mathrm{~kg}$ metal oxide inventory ( 100 seconds of simulation time).

Fig. 10. 3: Mole fraction of flue gases for Design 1 ( 100 seconds of simulation time).....

Fig. 10. 4: Time varying metal oxide outlet mass flow rate for Design 1, $20 \mathrm{~kg}$ inventory ( 75 seconds of simulation time).

Fig. 10. 5: Time varying carbon outlet mass flow rate for Design 1, $20 \mathrm{~kg}$ inventory ( 75 seconds of simulation time).

Fig. 10. 6: Design 2 for a char-metal oxide test FR.

Fig. 10. 7: 2D and 3D mesh used in the analysis.

Fig. 10. 8: Two different reactor sizes tested. Diameters in Case 2 are double of Case 1. The mass in section 2 was constant at around $21 \mathrm{~kg}$. 133

Fig. 10. 9: Instantaneous metal oxide volume fraction. 135

Fig. 10. 10: Instantaneous char volume fraction. 135

Fig. 10. 11: Instantaneous volume fraction, Case 1, $10 \mathrm{~kg}$ inventory ( 175 seconds of simulation time).

Fig. 10. 12: Instantaneous volume fraction, Case 1, $5 \mathrm{~kg}$ inventory ( 175 seconds of simulation time). 137 $\mathrm{x}$ 
Fig. 10. 13: Instantaneous $\mathrm{MeO}$ volume fraction, $20 \mathrm{~kg} \mathrm{MeO}$ inventory ( 175 seconds of simulation time).

Fig. 10. 14: Instantaneous char volume fraction ( 175 seconds of simulation time)..... 138

Fig. 10. 15: Velocity Vectors for $0^{\circ}$ case ( 175 seconds of simulation time). 139

Fig. 10. 16: Velocity Vectors for $60^{\circ}$ case ( 175 seconds of simulation time).

Fig. 10. 17: Different compartments in FR for Design 2 (Figure not to scale). The red arrows indicate the flow of char and combustible gases. The blue arrows indicate flow of metal oxide. 141

Fig. 10. 18: Geometry used in the analysis (shown to scale). 141

Fig. 10. 19: Volume fraction and bed heights at $\sim 95$ seconds of simulation time. 145

Fig. 10. 20: Mole fraction of flue gases at $~ 95$ seconds. 146

Fig. 10. 21: The gas temperature and gas pressure at $\sim 95$ seconds. 146 


\section{List of Tables}

Table 3. 1: Quantities assumed in present analysis and that by Lyngfelt et al. (2001)............................ 9

Table 3. 2: Mass and energy balance for control volume 1 (i.e. Fuel reactor) .........................................17

Table 3. 3: Mass and energy balance for control volume 2 (i.e. Air reactor) .......................................... 18

Table 3. 4: Output quantities calculated from thermodynamic model. ..................................................2 21

Table 5. 1: Details of system properties for experiments of Goldschmidt et al. (2003)...........................36

Table 5. 2: Details of numerics for simulations of Goldschmidt et al. (2003) experiments. ......................36

Table 5.3: System properties for Moritomi et al. (1983) experiments.................................................. 40

Table 5.4: Numerics used for simulation of Moritomi et al. (1983) experiments. .................................. 40

Table 7. 1: System properties for experiments of Son and Kim (2006)...............................................5

Table 7. 2: System properties for experiments of Mattisson et al. (2001) .............................................54

Table 7. 3: Summary of reaction rates used for simulation...............................................................60

Table 7. 4: Details of coarse and fine grids for simulations of Son and Kim (2006) experiments. .............63

Table 7. 5: Numerics for simulation of Mattisson et al. experiments (2001) .........................................64

Table 8. 1: Simulated Fuel Reactor Properties (Leion et al., 2007)........................................................ 78

Table 8. 2: Mass fraction of different elements in char and tar ............................................................ 79

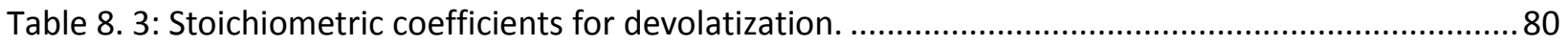

Table 8. 4: Numerical values for coefficients in gasification reactions (Everson et al., 2006). ..................81

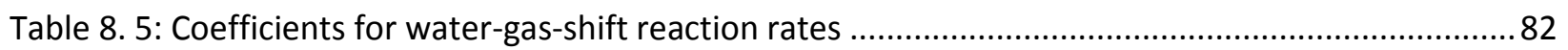

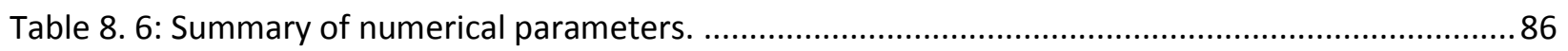

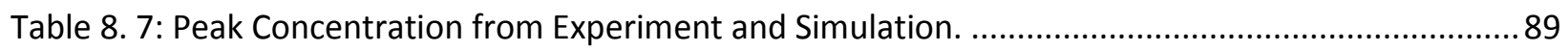

Table 8. 8: Computational mass balance of carbon in reactor. .......................................................... 91

Table 9. 1: System properties for experiments of Kronberger et al. (2004)...........................................96

Table 9. 2: Numerics for simulation of Kronbeger et al. (2004) experiments. ........................................97

Table 9. 3: Comparison of experimental and numerical simulation (time averaging for 4 seconds)....... 100 xii 
Table 9. 4: System properties for experiments of Abad et al. (2006).

Table 9. 5: Numerics for simulation of Abad experiments (2004)...... 105

Table 9. 6: Mass of metal oxides (grams).

Table 10. 1: Carbon capture efficiency and percentage combustion in some published experimental studies.

Table 10. 2: Comparison of char leakage and percentage combustion for Design 1. 130

Table 10. 3: Comparison of char leakage and percentage combustion for case 1 and case 2.

Table 10. 4: Comparison of char leakage and percentage combustion with increase in solid inventory.136

Table 10. 5: Comparison of char leakage and percentage combustion for different cone angles. 140

Table 10. 6: Char leakage and percentage combustion for Design 3. 145

Table 10. 7: Comparison of char leakage and percentage combustion for case 1 and case 2 . 148

xiii 


\section{Acknowledgements}

I am extremely grateful to my advisor Prof. John Kuhlman for providing me an opportunity to carry out this research work under his guidance and for providing continuous help throughout my Ph.D. studies.

Huge thanks to my supervisors at National Energy Technology Lab (NETL) Dr. Thomas O'Brien and E. David Huckaby for many technical inputs on the project. Their guidance has been critical to the success of this work.

Many thanks to my Ph.D. committee members Prof. Ismail Celik, Prof. Eric Johnson and Prof. Richard Turton for providing technical guidance on the research work.

A special thanks to my managers at ANSYS Inc. Dr. Karl Kuehlert, Stefano Orsino and Dave Looman for being very supportive of this research project as well as my Ph.D. studies at West Virginia University.

Thanks to Dr. Joe Mei, Dr. Lawrance Shadle and Douglas Straub of NETL for guidance on design of coal CLC systems.

Thanks to Dr. William Rogers (NETL) for supporting and funding this interesting research project.

Thanks to my colleagues at ANSYS Inc. Dr. Gilles Eggenspeiler, Dr. Jens Madsen, Dr. Shaoping Shi and Dr. Mehrdad Shahnam for their support and help at various times.

Thanks to my friends Keith Ruckman and Ashish Bambal, for their friendship, support and help.

My heartfelt thanks to my Parents Dr. Krishnaji Rao and Prema Rao, my Sister Dr. Kripa Rao Patil, my Brother-in-law Dr. Sachin Patil and my niece Saanvi for their constant motivation, affection and support.

The financial support of the U.S. Department of Energy, Carbon Sequestration and Gasification Programs administered at NETL is gratefully acknowledged.

I am also thankful for the scholarship and loans received from the J.N. Tata endowment for my graduate studies.

xiv 


\section{Chapter 1: Introduction}

There is an increase in the average temperature of the Earth's near-surface air and oceans since the mid-20th century and this increase is projected to continue for the next few decades. Increasingly the rise in temperatures is attributed primarily to release of greenhouse gases resulting from human activity such as burning of fossil fuels. The greenhouses gases such as $\mathrm{CO}_{2}$ and $\mathrm{CH}_{4}$ absorb thermal radiation from the sun and cause the Earth's overall temperature to be higher. The increase in global temperature is projected to cause an increase in sea levels and also change the amount and pattern of precipitation. It is also likely to cause the melting of glaciers as well as increases in the intensity of extreme weather events, species extinctions, and changes in agricultural yields.

Due to the threat of global warming, immense importance is being placed on developing technologies for producing power without the release of greenhouse gases such as $\mathrm{CO}_{2}$. Given the absence of credible alternative sources, the use of fossil fuels is still essential. Therefore new ways to reduce $\mathrm{CO}_{2}$ emissions from combustion of fossil fuels have to be developed. Presently several technologies, including oxy-fuel combustion, post-combustion capture from flue gases, carbon shift, etc., are being demonstrated (Simbeck, 1998) for $\mathrm{CO}_{2}$ capture. However these technologies will lead to significant increases in the cost of electricity, consuming a large portion of the energy they generate to separate gases (Simbeck, 1998; Chisea et al., 1998; Bolland, 1998; Audus et al., 1998).

A new combustion technology that has recently gained increased attention is chemical looping combustion (CLC) (Knocke and Richter, 1968; Richter and Knoche, 1983; Ishida et al., 1987, 1994; Lyngfelt et al., 2001; Ryu et al., 2001; Andres et al., 2005). The CLC system consists of two fluidized bed reactors, an air reactor (AR) and a fuel reactor (FR) (See Figure 1.1). The fuel is oxidized in the FR by using the reduction of granular metal oxides. Solid fuels can be used, although most of the reported work to date utilizes gaseous fuels, which can react directly with the carrier. In the case of solid fuels, it first devolatilizes and then is gasified by the recycled, fluidizing $\mathrm{H}_{2} \mathrm{O} / \mathrm{CO}_{2}$ in the $\mathrm{FR}$. The devolatilization and gasification products ( $\mathrm{CO}$ and $\mathrm{H}_{2}$ ) are then oxidized by the hot metal oxide. The reduced metal particles are returned to the AR where they are re-oxidized by air. The AR is typically a transport reactor. At its exit of the AR, the oxidized carrier is separated by a cyclone and returned to the FR. The net chemical reaction and energy release is identical to that of the conventional combustion of the fuel. The energy spent on solid circulation (the only energy cost of separation) is very small ( $0.3 \%$ ) in comparison with the total energy released (Lyngfelt, et al., 2001). The exhaust stream of the FR consists, mainly, of 
$\mathrm{CO}_{2}$ and $\mathrm{H}_{2} \mathrm{O}$. The $\mathrm{H}_{2} \mathrm{O}$ can be easily condensed resulting in a pure $\mathrm{CO}_{2}$ gas which can then be pressurized and sequestered. CLC holds significant promise as a next generation combustion technology as it has the potential to allow near-zero $\mathrm{CO}_{2}$ emissions with only minor effect on the overall efficiency of power plants and, hence, electricity cost (Ishida et al., 1996). Several energy and exergy analyses of CLC systems have been carried out, suggesting that power plant efficiencies greater than $50 \%$ can be achieved along with nearly complete $\mathrm{CO}_{2}$ capture (Ishida et al., 1987; Anheden, et al., 1995; Wolf, et al., 2001; Marion, 2006, Andrus, et al., 2006).
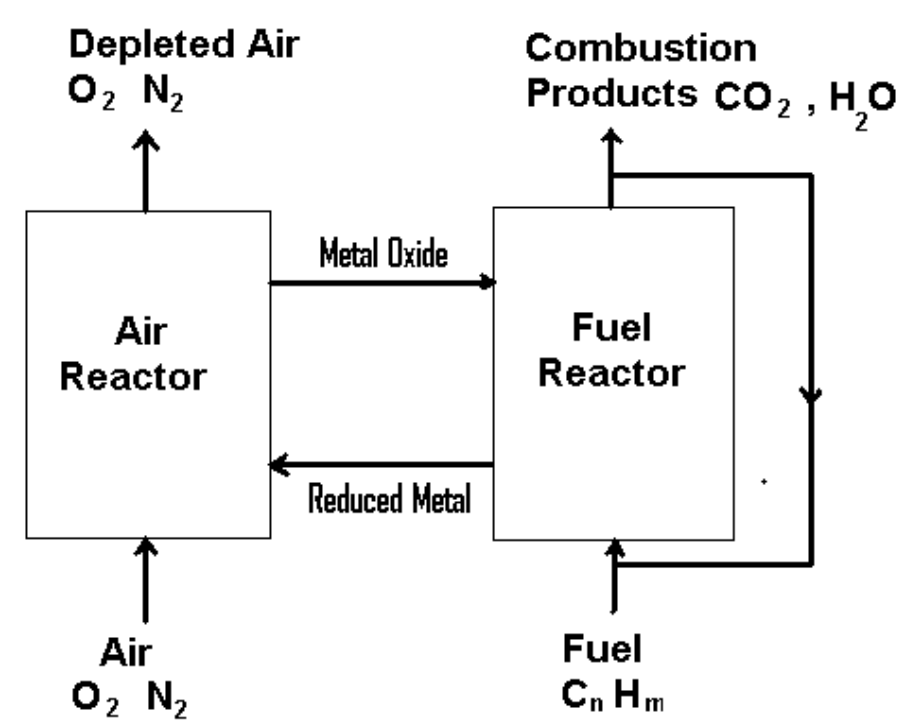

Fig. 1.1: Schematic of Chemical Looping Combustion (CLC) system (Lyngfelt et al., 2001)

Significant research is currently underway in the design of CLC reactors, and several small scale (300W to $120 \mathrm{~kW}$ ) test plants have been constructed (Lyngfelt and Thunman, 2005; De Deigo et al., 2007; Son and Kim, 2006; Kolbitsch et al., 2009). Also, there has been extensive study of the properties of carriers, an important aspect of CLC technology. It is critical that the rates of reaction of the carrier, both reduction by the fuel and oxidation by air, be high at the operating temperature of the reactors. Also, it is desirable to have a low attrition rate to minimize "carrier make-up" costs, a potentially significant operating cost of this $\mathrm{CO}_{2}$ separation process. It should also be economical and environmentally benign. Various studies have been carried out to characterize the reduction and oxidation behavior of metal oxides with various gases such as $\mathrm{CO}, \mathrm{H}_{2}, \mathrm{CH}_{4}$ (Johansson et al., 2004; Ryu et al., 2001; Ishida et al., 1996; Mattisson et al., 2001; Adánez et al., 2004, Siriwardane et al., 2007, 
Chandel et al., 2009). Models based on shrinking core and changing grain size have been used to accurately represent the chemical kinetics of the metal oxides.

CFD provides the means to analyze the interaction between fluid mechanics and chemical kinetics. Fluid mechanical effects, such as bubble formation in the FR and fuel leakage into the air reactor, could affect the performance of the reactor. Despite the large number of publications in the past few years on the experimental studies of CLC, very little attention has been paid to CFD studies of such systems. Jung and Gamwo (2008) and Deng et al. (2009), have performed CFD studies of FR using gaseous fuels. No CFD simulations involving solid fuels have been previously reported. In the present study we present primarily two-dimensional CFD simulations of CLC systems, along with more limited three-dimensional simulations, and demonstrate the ability of multi-phase CFD to accurately simulate recent experiments. We then use the validated CFD model to evaluate designs for CLC systems using solid fuels (coal, modeled as char). 


\section{Chapter 2: Carbon Capture and Storage Technologies}

In the previous chapter the global warming phenomenon and its potential harmful effects have been summarized. It is becoming increasingly necessary to reduce or all together eliminate the release of $\mathrm{CO}_{2}$ to the atmosphere. Presently the reliance on fossil fuels as an energy source is significant. In many countries such as the US; the fossil fuels provide close to $50 \%$ of the total energy. Given the absence of any credible alternative source of energy, the use of fossil fuels as a source of energy will continue for a long time to come. It is therefore necessary to develop technologies which will be able to capture the release of $\mathrm{CO}_{2}$ from large concentrated sources such as power plants and then dispose or store the $\mathrm{CO}_{2}$ in a safe manner. This chapter briefly describes various available technologies to capture and store the $\mathrm{CO}_{2}$ in a cost effective and reliable way.

\subsection{Carbon Storage Techniques}

Before technologies for carbon capture are developed, there is a need to make sure that the captured $\mathrm{CO}_{2}$ can be stored in a safe manner. Some of the technologies that have been proposed for this purpose are discussed below.

Geological storage: This is also known as geosequestration. In this case the $\mathrm{CO}_{2}$ is stored in used oil, gas fields and deep un-minable coal beds. Significantly more amount of $\mathrm{CO}_{2}$ can be stored in depleted gas fields in comparison with the $\mathrm{CO}_{2}$ obtained from burning the gas from these wells.

In the case of oil fields $\mathrm{CO}_{2}$ can be pumped into wells to enhance the oil recovery. This technology is useful as the geology of the oil wells is well understood and the cost of recovered oil can offset the cost of sequestration. The generated $\mathrm{CO}_{2}$ can also be stored in coal beds where they can be absorbed in the pore matrix and in turn releasing trapped methane which can be then collected and sold.

Ocean storage: At a depth of $500 \mathrm{~m}$ of water (or 50 bars) $\mathrm{CO}_{2}$ becomes liquid. However the density of this liquid is slightly less than the surrounding water and hence it slowly rises. If proper measures are taken to mix the liquid $\mathrm{CO}_{2}$ with water then the mixture will have a higher density and will sink.

At a depth of $3000 \mathrm{~m}$ below sea level, the density of $\mathrm{CO}_{2}$ is higher than sea water and at these depths it forms lakes of $\mathrm{CO}_{2}$ and is likely to remain there for a long time. There are likely to be some 
environmental effects due to high acidity in the region as well as the fact that the high $\mathrm{CO}_{2}$ concentration could kill ocean organisms.

Mineral storage: The $\mathrm{CO}_{2}$ can be made to react with minerals containing Calcium, Magnesium etc to form carbonates. The carbonates are stable compounds and are likely to retain $\mathrm{CO}_{2}$ permanently. However the major challenge is to find suitable minerals where the reaction rate with $\mathrm{CO}_{2}$ is high enough so that the process becomes feasible.

Another important aspect of carbon capture is the transportation of $\mathrm{CO}_{2}$ from power plants to the storage area. The $\mathrm{CO}_{2}$ can be transported to suitable storage sites through pipelines. In case of deep sea storage, ships can also be used. Presently $\mathrm{CO}_{2}$ is already transported in the US to oil fields for enhanced oil recovery.

\subsection{Carbon Capture Techniques}

The carbon capture technologies can be divided into four main areas: (i) post-combustion capture, (ii) oxy-fired combustion, (iii) CO-shift and (iv) direct techniques such as CLC.

Post Combustion capture: In post combustion capture the $\mathrm{CO}_{2}$ is removed from the flue gas. The combustion process itself is not affected. The energy required for $\mathrm{CO}_{2}$ separation is provided by the power generation process. There exist several well proven technologies for post combustion capture. Some examples include the use of a physical absorbent such as mono-ethanolamine which absorbs $\mathrm{CO}_{2}$ from the flue gases or the use of cryogenic separation techniques.

Oxy-Fired combustion: In this case a pure stream of oxygen is used to combust the fuel. This results in a pure $\mathrm{CO}_{2}$ stream at the exit which can be directly sequestrated without the need to separate out the $\mathrm{CO}_{2}$. However energy has to be spent on separating the pure oxygen stream from Air. Also the combustion temperatures for oxy-fired systems are significantly higher than the regular combustion process and hence the technology to handle gases at high temperature and to extract work from them has to be developed.

CO-shift combustion: In this case the fuel is first gasified and converted to steam gas which is essentially a mixture of $\mathrm{CO}$ and $\mathrm{H}_{2}$. The $\mathrm{CO}$ is then treated with high temperature steam to convert it to $\mathrm{CO}_{2}$ and $\mathrm{H}_{2}$. The $\mathrm{CO}_{2}$ is then separated from $\mathrm{H}_{2}$, and the $\mathrm{H}_{2}$ can be directly used as fuel. 


\section{3: Cost comparisons for various carbon capture technologies}

Figure 2.1 shows the efficiency penalties from various carbon capture technology, as estimated from various sources. The efficiency penalty is defined as:

Energy penalty $=1-\frac{\text { Efficiency with separation }}{\text { Efficiency without separation }}$

It is clear that various estimates suggest that there is likely on an average at least a $15 \%$ efficiency penalty due to carbon capture.

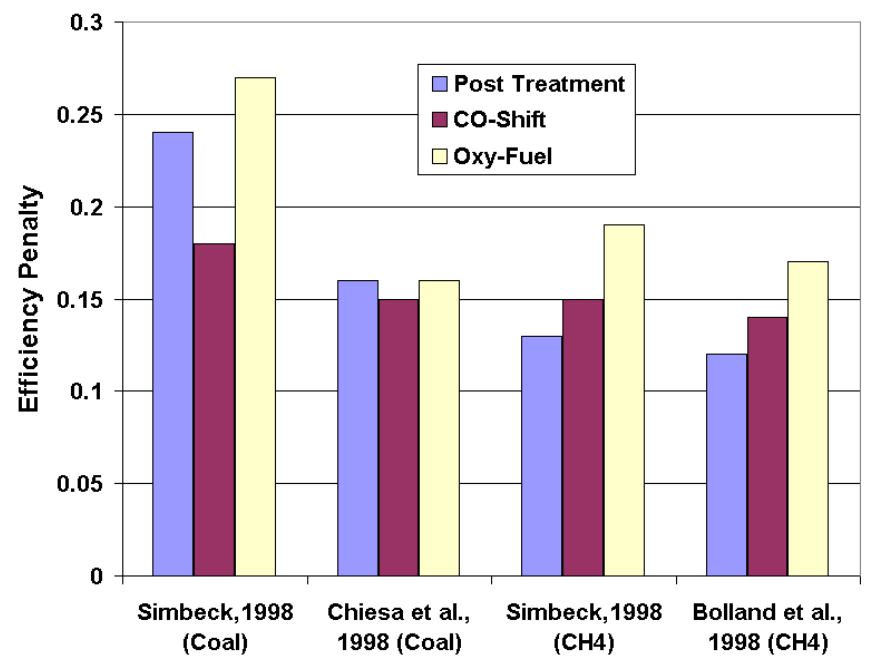

Fig. 2.1: Efficiency penalties for various carbon capture technologies.

Figure 2.2 shows the estimated cost penalties of carbon capture. The cost penalty is defined as:

Cost penalty $=\frac{\text { Cost With Separation }}{\text { Cost Without Separation }}-1$

It is clear that on an average the cost penalties are likely to be higher by almost 30 to $70 \%$. 


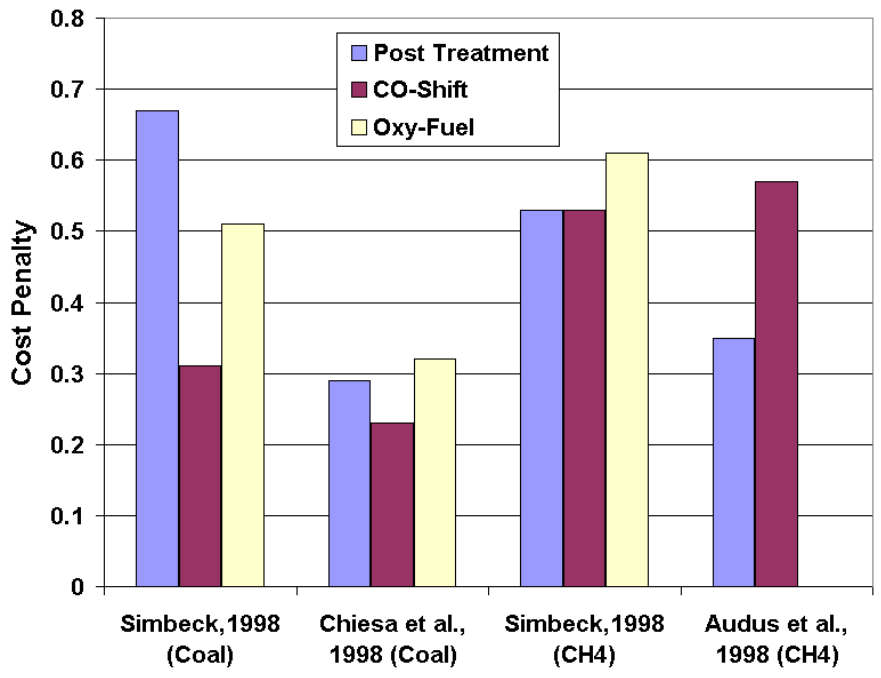

Fig. 2.2: Cost penalties for various carbon capture technologies.

As was discussed in chapter 1 the use of CLC provides a direct sequestration-ready $\mathrm{CO}_{2}$ stream in a natural fashion without the need to spend any energy in gas-separation processes. Therefore it is likely that the cost increases due to use of CLC will be significantly lower in comparison with other carbon capture technologies. This is likely to be a major advantage for CLC. 


\section{Chapter 3: Thermodynamic Cycle Analysis for Design of CLC}

One of the goals of this research work is to develop basic design relationships for a chemical looping combustion demonstration facility that will be built at National Energy Technology Lab, Morgantown, WV. CLC requires many unit operations involving gas-solid or granular flow. A CLC system generally consists of two reactors, a Fuel Reactor (FR) and an Air Reactor (AR). The FR is a critical component in the CLC system. It is desirable that it completely oxidizes the fuel as well as provides a concentrated $\mathrm{CO}_{2}$ stream which allows a high amount of carbon capture. The complexities of coal combustion in the FR have been discussed in Chapter 1. In comparison the AR which re-oxidizes the reduced carrier is typically a transport reactor, which is simpler to design. The question of how a CLC fuel reactor should be designed that will allow complete combustion of fuel and high carbon capture efficiencies will be looked into in Chapter 10. Before the detailed design of FR is carried out, the basic design relationships have to be formulated. In this chapter equations are developed that will allow the preliminary design of a CLC system. The first step is to clearly identify the input and the output quantities of interest. These are listed below:

Inputs for Thermodynamic analysis include the following:

1) Power required from the CLC system

2) Maximum temperature allowed in the Air Reactor (This is limited by the sintering temperature of the metal oxides.)

3) Type of fuel and its properties as well as properties of metal oxide used

The outputs from Thermodynamic analysis are:

1. Fuel flow rate

2. Air flow rate

3. Cross-sectional area of FR

4. Cross-sectional area of $A R$

5. Superficial velocity of FR 
6. Superficial velocity in $A R$

7. Metal oxide to metal reduction extent

8. Bed height in FR

9. Solid circulation rate for thermal balance

The procedure followed below is similar to that of Lyngfelt et al. (2001) but with important modifications. Lyngfelt et al. assume values for several quantities that may be difficult to ascertain a priori. The Table 3.1 compares the quantities that were assumed by Lyngfelt et al., with those in the present analysis. The analysis of Lyngfelt et al. does not involve the energy balance of the reactors, instead operating temperatures of the two reactors are directly assumed. Jerndal et al. (2006) and Kronberger et al. (2005) have carried out energy balance of fuel and air reactor separately. They do not account for the strong coupling of both energy and mass between the air and fuel reactor. In the present analysis both energy and mass balance is solved iteratively in a coupled fashion.

Table 3.1: Quantities assumed in present analysis and that by Lyngfelt et al. (2001).

\begin{tabular}{|l|l|l|}
\hline & Is it Assumed by Lyngfelt? & $\begin{array}{l}\text { Is it Assumed in Present } \\
\text { Analysis? }\end{array}$ \\
\hline Power Requirement (Fuel) & Yes & Yes \\
\hline Flue Gas Oxygen & Yes & Yes \\
\hline Conversion Difference & Yes & No \\
\hline Oxidizer Cross Section & Yes & No \\
\hline Reducer Cross Section & Yes & No \\
\hline Temperature Oxidizer & & $\begin{array}{l}\text { Yes (Assumed equal to } \\
\text { sintering temperature) }\end{array}$ \\
\hline Temperature Reducer & Yes & No \\
\hline Pressure Drop, Oxidizer & Yes & No \\
\hline Pressure Drop Reducer & Yes & No \\
\hline Air Reactor Cross-section & Yes & No \\
\hline Fuel Reactor Cross-section & Yes & No \\
\hline
\end{tabular}




\subsection{Theory:}

Given the type of fuel and the power requirements, the fuel flow rate required can be computed using the following equation:

$$
\dot{m}_{f}=\frac{\text { Power }}{H V_{i}}
$$

Here $\dot{m}_{f}$ is the mass flow rate of the fuel and $H V_{i}$ is the lower heating value of the fuel.

The following reaction for complete combustion of hydro-carbon fuel is assumed:

$v_{a} \mathrm{Fuel}+v_{b} \mathrm{O}_{2} \rightarrow v_{c} \mathrm{H}_{2} \mathrm{O}+v_{d} \mathrm{CO}_{2}$

where $v_{a}$ and $v_{b}$ are the stoichiometric coefficients.

The amount of oxygen required for combustion $\left(\dot{m}_{\mathrm{O}_{2}}\right)$ is then given as:

$\dot{m}_{\mathrm{O}_{2}}=M W_{\mathrm{O}_{2}} \frac{\dot{m}_{\text {Fuel }}}{M W_{\text {Fuel }}} \frac{v_{b}}{v_{a}}$

where $M W_{\text {Fuel }}$ is the molecular weight of the fuel and $M W_{O_{2}}$ is the molecular weight of oxygen.

Assuming some value of air ratio $\left(\lambda_{\text {air }}\right)$ one can calculate the amount of air flow rate that is required. Air ratio is essentially a measure of extra air above the stoichiometric value that has to be supplied (e.g. $20 \%$ or $\lambda_{\text {air }}=1.2$ ).

$\dot{m}_{\text {Air }}=\frac{\dot{m}_{O_{2}} \lambda_{\text {air }}}{0.233}$

The suggested operating condition for the FR has been the bubbling regime (Lyngfelt et al., 2001; Son and Kim, 2006). To operate in the bubbling regime the superficial fluidizing velocity has to be several times the minimum fluidization velocity. The minimum fluidization velocity can be calculated from a quadratic equation derived from the well known Ergun equation (Kunii and Levenspiel, 1991): 
$\frac{1.75}{\varepsilon_{m f}^{3} \phi_{s}}\left(\frac{d_{p} u_{m f} \rho_{g}}{\mu}\right)^{2}+\frac{150\left(1-\varepsilon_{m f}\right)}{\varepsilon_{m f}^{3} \phi_{s}^{2}}\left(\frac{d_{p} u_{m f} \rho_{g}}{\mu}\right)=\frac{d_{p}^{3} \rho_{g}\left(\rho_{s}-\rho_{g}\right) g}{\mu^{2}}$

The superficial velocity in the fuel reactor is taken to be:

$u_{F R}=n_{1} u_{m f}$

where $n_{1}$ is a suitable real number, such as 5 .

The air reactor has to transport the particles back to the fuel reactor and hence the velocity in the air reactor has to be several times the terminal velocity of the particle. The particle terminal velocity can be calculated by balancing the drag with buoyancy and gravity forces.

$\frac{C_{D} u_{t}^{2} A_{p} \rho_{g}}{2 m}=g \frac{\rho_{s}-\rho_{g}}{\rho_{s}}$

The terminal velocity can then be calculated as:

$u_{t}=\sqrt{\frac{2 g\left(\rho_{p}-\rho_{g}\right) m}{A_{p} \rho_{s} C_{D} \rho_{g}}}$

Here $m$ is the particle mass and $C_{D}$ is the single particle drag coefficient for which several different particle drag correlations are available in literature. In the present case the drag coefficient suggested by Haider and Levenspiel (1989) is used in equation 3.8 and which is also given by Kunii and Levenspiel, 1991:

$C_{D}=\frac{24}{\operatorname{Re}}+3.3643 \mathrm{Re}^{0.3471}+\frac{0.4607 \mathrm{Re}}{\operatorname{Re}+2682.5}$

The superficial velocity in the air reactor is now taken to be:

$u_{A R}=n_{2} u_{t}$

where $n_{2}$ is some assumed real number such as 3 .

A high solid circulation rate is required for two purposes:

11 
1) To supply enough oxygen to combust all the fuel.

2) To supply enough heat to maintain endothermic reactions in the fuel reactor.

The oxygen carrying capacity $\left(R_{o}\right)$ of metal oxide (the amount of oxygen available per unit metal oxide mass) can now be defined as:

$R_{o}=\frac{M W_{o x}-M W_{r e d}}{M W_{o x}}$

where $M W_{o x}$ is the molecular weight of the oxidized form of the metal oxide and $M W_{\text {red }}$ is the molecular weight of the reduced form of the metal oxide.

The difference between the oxidized and reduced form is the mass of oxygen that is available to be transferred. Now assume that the average conversion of metal oxide in the air reactor is given as $X_{A R}$ and average conversion of metal oxide in fuel reactor is $X_{F R}$, so that the required metal oxide mass flow rate to supply enough oxygen will be given as:

$\dot{m}_{\text {Solids }}=\frac{\dot{m}_{O_{2}}}{R_{o} \Delta X}$

where $\Delta X=X_{A R}-X_{F R}$

$X_{A R}$ is likely to be close to 1 in the air reactor; the exact value is not particularly important. The value of $X_{F R}$ is calculated based on an iterative technique which uses the energy balance.

Equations are now determined for the energy balances in the fuel reactor and the air reactor that can be used for determining the temperature and required solid flow rates in the two reactors. First consider Control Volume 1 (FR) shown in the Fig. 3.1. There are two inlet streams, fuel and oxidized metal oxide, and two outlet streams, reduced metal oxide \& exhaust gases. In case solid fuel is used then an extra inlet stream consisting of steam is required for gasifying the fuel. 


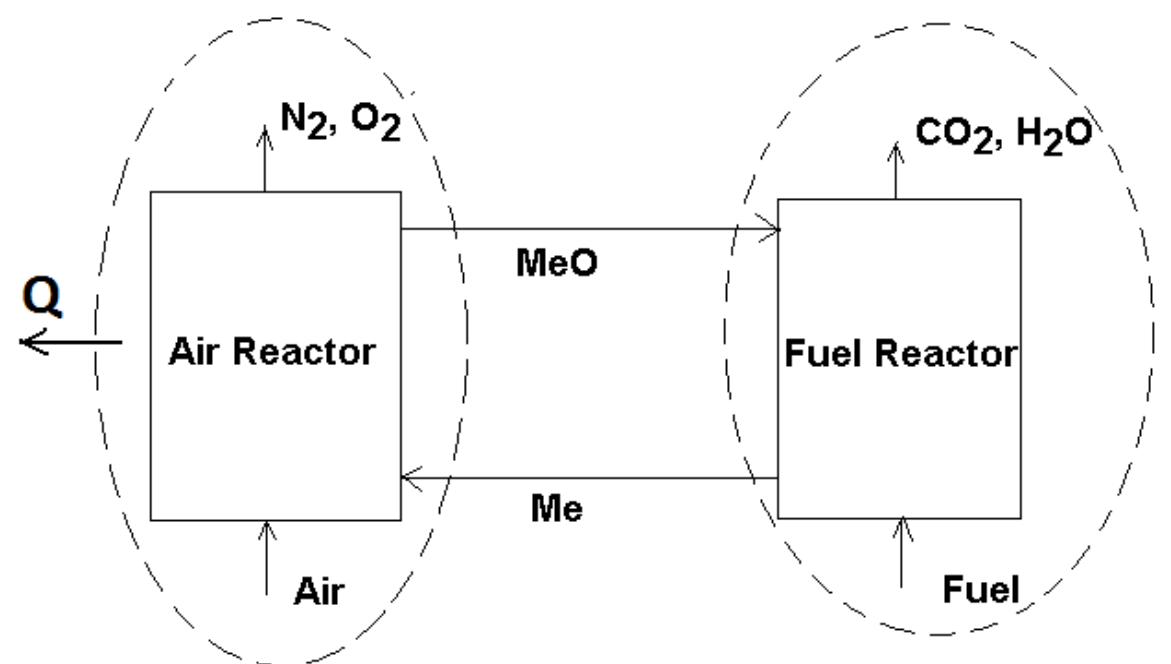

CONTROL VOLUME 2

CONTROL VOLUME 1

Fig. 3.1: Control volumes used for energy balance of AR and FR reactors.

The mass balance can be written as:

$\dot{m}_{\text {solid_in }}-\dot{m}_{\text {solid_out }}+\dot{m}_{\text {fuel }}-\dot{m}_{\mathrm{CO} 2}-\dot{m}_{\mathrm{H} 2 \mathrm{O}}=0$

The energy balance for control volume 1 is given as:

$$
\begin{aligned}
& \dot{m}_{\text {solid_in }}\left(\Delta H_{f 298}^{\circ}(\text { solid_in })+\int_{T_{298}}^{T_{A R}} C_{p}^{\circ}(\text { solid_in }) d T\right) \\
& -\dot{m}_{\text {solid_out }}\left(\Delta H_{f 298}^{\circ}\left(\text { solid } \_ \text {out }\right)+\int_{T_{298}}^{T_{F R}} C_{p}^{\circ}(\text { solid_out }) d T\right) \\
& +\dot{m}_{f u e l}\left(\Delta H_{f 298}^{\circ}(\text { fuel })+\int_{T_{298}}^{T_{F R}} C_{p}^{\circ}(\text { fuel }) d T\right) \\
& -\dot{m}_{C O_{2}}\left(\Delta H_{f 298}^{\circ}\left(C O_{2}\right)+\int_{T_{298}}^{T_{F R}} C_{p}^{\circ}\left(C O_{2}\right) d T\right) \\
& -\dot{m}_{H_{2} O}\left(\Delta H_{f 298}^{\circ}\left(H_{2} O\right)+\int_{T_{298}}^{T_{F R}} C_{p}^{\circ}\left(H_{2} O\right) d T\right)=0
\end{aligned}
$$

where $T_{r e f}$ is the reference temperature at which the enthalpy of formation is known $\left(T_{r e f}=298 \mathrm{~K}\right)$. Usually during the combustion of fossil fuels, large amount of energy is released due to conversion of reactant/fuel into products of combustion. The difference between the enthalpy of formation of reactants and products contributes to the bulk of the released energy. For example in the combustion of methane at $1200 \mathrm{~K}$, the difference in enthalpy of formation of the reactant (methane) and products $\left(\mathrm{CO}_{2}\right.$ and $\mathrm{H}_{2} \mathrm{O}$ ) is more than $90 \%$ of the total released energy. The energy associated with heating or cooling of the reacting species (given by $\int C_{p}^{o} d T$ ) is usually small. Therefore in the present analysis, the 
relatively small contribution of the heat energy associated with specific heat capacity is approximated as $\int C_{p}^{\circ} d T=\bar{C}_{p} d T$, where $\bar{C}_{p}$ is the specific heat capacity calculated at the average of $\mathrm{T}_{\text {ref }}$ and operating temperature of the reactor i.e. $\left(T_{\text {ref }}+T_{\text {rector }}\right) / 2$.

A full conversion from $\mathrm{MeO}$ to $\mathrm{Me}$ and back to $\mathrm{MeO}$, is not necessarily obtained in a real system. Therefore the mass flow rate of solid can be divided into the oxidized and reduced parts as:

$$
\dot{m}_{\text {solid_in }}=\dot{m}_{\text {ox_in }}+\dot{m}_{\text {red_in }}
$$

The oxidized and reduced forms of metal oxides have different properties such as the enthalpy of formation, specific heat, etc. Therefore they have to be treated separately.

Therefore the inlet energy of the solid can be calculated as:

$$
\begin{aligned}
& \dot{m}_{\text {solid_in }}\left(\Delta H_{f 298}^{\circ}(\text { solid_in })+\int_{T_{298}}^{T_{A R}} C_{p}^{\circ}(\text { solid_in }) d T\right)= \\
& \dot{m}_{\text {ox_in }}\left(\Delta H_{f 298}^{\circ}(\text { oxdized form })+\int_{T_{298}}^{T_{A R}} C_{p}^{\circ}(\text { oxdized form }) d T\right) \\
& +\dot{m}_{\text {red_in }}\left(\Delta H_{f 298}^{\circ}(\text { reduced form })+\int_{T_{298}}^{T_{A R}} C_{p}^{\circ}(\text { reduced form }) d T\right)
\end{aligned}
$$

The mass of oxidized fraction and reduced fraction of solid entering and leaving the fuel reactor now must be found. The relationship between inlet mass flow of solid and exit mass flow of solid can be found by the fact that the mass of the two streams in the fully oxidized condition should be the same.

$\dot{m}_{\text {solid_in }}($ When Fully Oxidized $)=\dot{m}_{\text {solid_out }}($ When Fully Oxidized $)$

$$
X_{A R}=\frac{\dot{m}_{\text {solid_in }}-\dot{m}_{r e d}}{\dot{m}_{o x}-\dot{m}_{r e d}}
$$

where $\dot{m}_{o x}$ and $\dot{m}_{\text {red }}$ is the mass flow of metal oxide if it were in fully oxidized and reduced state respectively.

Similarly

$$
X_{F R}=\frac{\dot{m}_{\text {solid_out }}-\dot{m}_{\text {red }}}{\dot{m}_{\text {ox }}-\dot{m}_{\text {red }}}
$$

Now $R_{o}=\frac{\dot{m}_{o x}-\dot{m}_{r e d}}{\dot{m}_{o x}}$ or $\dot{m}_{r e d}=\left(1-R_{o}\right) \dot{m}_{o x}$

Substitution of equation 3.19 in equation 3.18 yields 
$\dot{m}_{o x}=\frac{1}{\left(1-R_{o}+X_{A R} R_{o}\right)} \dot{m}_{\text {solid_in }}$

Also $\dot{m}_{\text {red }}=\frac{\left(1-R_{o}\right)}{\left(1-R_{o}+X_{A R} R_{o}\right)} \dot{m}_{\text {solid_in }}$

The following linear relationship also holds for the oxidized and reduced portion of inlet solid mass to FR

$$
\dot{m}_{o x_{-} i n}=X_{A R} \dot{m}_{o x} \text { and } \dot{m}_{\text {red_in }}=\left(1-X_{A R}\right) \dot{m}_{r e d}
$$

The following linear relationships also hold for the oxidized and reduced portion of outlet solid mass from FR

$$
\dot{m}_{\text {ox_out }}=X_{F R} \dot{m}_{\text {ox }} \text { and } \dot{m}_{\text {red_out }}=\left(1-X_{F R}\right) \dot{m}_{\text {red }}
$$

Using equations $3.20,3.21$ and substituting in $3.22 \mathrm{a}$ and $3.22 \mathrm{~b}$ the following equation $3.23 \mathrm{a}$ and $3.23 \mathrm{~b}$ are obtained:

$$
\begin{aligned}
& \dot{m}_{\text {solid_in }}=\dot{m}_{\text {ox_in } i n}+\dot{m}_{\text {red_in }}=\frac{X_{A R}}{\left(1-R_{o}+X_{A R} R_{o}\right)} \dot{m}_{\text {solid_in }}+\frac{\left(1-X_{A R}\right)\left(1-R_{o}\right)}{\left(1-R_{o}+X_{A R} R_{o}\right)} \dot{m}_{\text {solid_in }} \\
& \dot{m}_{\text {solid_out }}=\dot{m}_{\text {ox_out }}+\dot{m}_{\text {red_out }}=\frac{X_{F R}}{\left(1-R_{o}+X_{A R} R_{o}\right)} \dot{m}_{\text {solid_in }}+\frac{\left(1-X_{F R}\right)\left(1-R_{o}\right)}{\left(1-R_{o}+X_{A R} R_{o}\right)} \dot{m}_{\text {solid_in }}
\end{aligned}
$$

Using equation 3.23a the equation 3.16 can now be evaluated. This leads us to the following energy equation for solid outflow:

$$
\begin{aligned}
& \dot{m}_{\text {solid_out }}\left(\Delta H_{f 298}^{\circ}(\text { solid_out })+\int_{T_{298}}^{T_{F R}} C_{p}^{\circ}(\text { solid_out }) d T\right) \\
& =\dot{m}_{\text {ox_out }}\left(\Delta H_{f 298}^{\circ}(\text { oxdized form })+\int_{T_{298}}^{T_{F R}} C_{p}^{\circ}(\text { oxdized form }) d T\right) \\
& +\dot{m}_{\text {red_out }}\left(\Delta H_{f 298}^{\circ}(\text { reduced form })+\int_{T_{298}}^{T_{F R}} C_{p}^{\circ}(\text { reduced form }) d T\right)
\end{aligned}
$$

Also let the reaction be defined by following stoichiometric coefficients:

MetalOxide $+v_{a}$ Fuel $\rightarrow v_{b} \mathrm{CO}_{2}+v_{c} \mathrm{H}_{2} \mathrm{O}+$ Reduced MetalOxide

$\dot{m}_{\mathrm{CO}_{2}}=\frac{v_{b} \times M W_{\mathrm{CO}_{2}}}{v_{a} \times M W_{f u e l}} \dot{m}_{f u e l}$

$\dot{m}_{\mathrm{H}_{2} \mathrm{O}}=\frac{v_{c} \times M W_{\mathrm{H}_{2} \mathrm{O}}}{v_{a} \times M W_{\text {fuel }}} \dot{m}_{\text {fuel }}$

The above equations give the complete set of equations to solve for energy (Equation 3.14). From Equation 3.14 we will obtain the temperature of fuel reactor. 
Now consider the Control Volume 2 (Air reactor) shown in the Fig. 3.1. The energy equation is:

$$
\begin{aligned}
& \dot{m}_{\text {solid_in }}\left(\Delta H_{f 298}^{\circ}(\text { solid_in })+\int_{T_{298}}^{T_{A R}} C_{p}^{\circ}(\text { solid_in }) d T\right) \\
& -\dot{m}_{\text {solid_out }}\left(\Delta H_{f 298}^{\circ}(\text { solid_out })+\int_{T_{298}}^{T_{F R}} C_{p}^{\circ}(\text { solid_out }) d T\right) \\
& +\dot{m}_{\text {air }}\left(\Delta H_{f 298}^{\circ}(\text { air })+\int_{T_{298}}^{T_{A R}} C_{p}^{\circ}(\text { air }) d T\right) \\
& -\dot{m}_{N_{2}}\left(\Delta H_{f 298}^{\circ}\left(N_{2}\right)+\int_{T_{298}}^{T_{A R}} C_{p}^{\circ}\left(N_{2}\right) d T\right)-\dot{m}_{O_{2}}\left(\Delta H_{f 298}^{\circ}\left(O_{2}\right)+\int_{T_{298}}^{T_{A R}} C_{p}^{\circ}\left(O_{2}\right) d T\right)=Q
\end{aligned}
$$

The equation sets solved for the FR (control volume 1) and AR (control volume 2) are given in Tables 3.2 and 3.3 respectively.

The iterative solution method is as follows (Also see Fig. 3.2 for flow chart):

1) Choose a $\Delta X$ value.

2) Calculate the mass flow rate of solids from equation 3.11. The oxygen mass flow $\dot{m}_{\mathrm{O}_{2}}$ is a known based on the amount of fuel that has to be combusted.

3) Take the temperature of the $A R\left(T_{A R}\right)$ to be the maximum allowable based on the metal oxides used. The maximum allowable temperature is around the sintering temperature. Beyond the sintering temperature the grain structure of the particle changes leading to a reduction in the porosity and reaction rates of the metal oxide. A high temperature is required for high thermodynamic efficiency and high exergy.

4) Solve the energy balance for FR/control volume 1 (See Fig. 3.2 and equation 3.14.) as shown below to obtain the temperature of FR $\left(T_{F R}\right)$.

5) Using the temperature of the FR, solve the energy balance for the air reactor (control volume 2) (equation 3.28) to recalculate the temperature of air reactor $\left(T_{A R}\right)$.

6) If the calculated temperature in the air reactor is higher than the assumed value, then increase $\Delta X$, if less than, decrease $\Delta X$ if the calculated temperature is less than the maximum possible. Repeat steps $2,3,4$ and 5 until the calculated air reactor temperature matches with the assumed value.

7) When the calculated and assumed air reactor temperature matches the consistent value of $\Delta X$ has been found. 
Table 3.2: Mass and energy balance for control volume 1 (i.e. Fuel reactor)

\begin{tabular}{|c|c|}
\hline \multicolumn{2}{|r|}{ Mass Balance } \\
\hline $\begin{array}{l}\text { Total Mass } \\
\text { Balance }\end{array}$ & $\dot{m}_{\text {solid_in }}-\dot{m}_{\text {solid_out }}+\dot{m}_{f u e l}-\dot{m}_{\mathrm{CO}_{2}}-\dot{m}_{\mathrm{H}_{2} \mathrm{O}}=0$ \\
\hline $\begin{array}{l}\text { Equation for } \\
\text { solid mass } \\
\text { transported } \\
\text { into the FR }\end{array}$ & $\begin{array}{l}\dot{m}_{\text {solid_in }}=\frac{\dot{m}_{\mathrm{O}_{2}}}{R_{o} \Delta X} \quad \text { where, } \dot{m}_{\mathrm{O}_{2}}=M W_{\mathrm{O}_{2}} \frac{\dot{m}_{\text {Fuel }}}{M W_{\text {Fuel }}} \frac{v_{b}}{v_{a}} \\
\text { The oxidized and reduced percentage of the solid mass into the FR is given as } \\
\dot{m}_{\text {solid_in }}=\dot{m}_{\text {ox_in }}+\dot{m}_{\text {red_in }}=\frac{X_{A R}}{\left(1-R_{o}+X_{A R} R_{o}\right)} \dot{m}_{\text {solid_in }}+\frac{\left(1-X_{A R}\right)\left(1-R_{o}\right)}{\left(1-R_{o}+X_{A R} R_{o}\right)} \dot{m}_{\text {solid_in }}\end{array}$ \\
\hline $\begin{array}{l}\text { Equation for } \\
\text { solid mass } \\
\text { transported } \\
\text { out of FR }\end{array}$ & $\begin{array}{l}\text { The oxidized and reduced percentage of the solid mass out of the FR is given as } \\
\dot{m}_{\text {solid_out }}=\dot{m}_{\text {ox_out }}+\dot{m}_{\text {red_out }}=\frac{X_{F R}}{\left(1-R_{o}+X_{A R} R_{o}\right)} \dot{m}_{\text {solid_in }}+\frac{\left(1-X_{F R}\right)\left(1-R_{o}\right)}{\left(1-R_{o}+X_{A R} R_{o}\right)} \dot{m}_{\text {solid_in }}\end{array}$ \\
\hline $\begin{array}{l}\text { Equation for } \\
\text { fuel flow rate }\end{array}$ & $\dot{m}_{f}=\frac{\text { Power }}{H V_{i}}$ \\
\hline $\begin{array}{l}\text { Equation for } \\
\mathrm{CO}_{2} \text { and } \mathrm{H}_{2} \mathrm{O} \\
\text { flow Rates }\end{array}$ & $\dot{m}_{\mathrm{CO}_{2}}=\frac{v_{b} \times M W_{\mathrm{CO}_{2}}}{v_{a} \times M W_{f u e l}} \dot{m}_{f u e l}$ and $\dot{m}_{\mathrm{H}_{2} \mathrm{O}}=\frac{v_{c} \times M W_{\mathrm{H}_{2} \mathrm{O}}}{v_{a} \times M W_{f u e l}} \dot{m}_{\text {fuel }}$ \\
\hline \multicolumn{2}{|r|}{ Energy Balance } \\
\hline $\begin{array}{l}\text { Total energy } \\
\text { balance }\end{array}$ & $\begin{array}{l}\dot{m}_{\text {solid_in }}\left(\Delta H_{f 298}^{\circ}(\text { solid_in })+\int_{T_{298}}^{T_{A R}} C_{p}^{\circ}(\text { solid_in }) d T\right) \\
-\dot{m}_{\text {solid_out }}\left(\Delta H_{f 298}^{\circ}(\text { solid_out })+\int_{T_{298}}^{T_{F R}} C_{p}^{\circ}(\text { solid_out }) d T\right)+\dot{m}_{f u e l}\left(\Delta H_{f 298}^{\circ}(f u e l)+\int_{T_{298}}^{T_{F R}} C_{p}^{\circ}(f u e l) d T\right) \\
-\dot{m}_{\mathrm{CO}_{2}}\left(\Delta H_{f 298}^{\circ}\left(\mathrm{CO}_{2}\right)+\int_{T_{298}}^{T_{F R}} C_{p}^{\circ}\left(C O_{2}\right) d T\right)-\dot{m}_{H_{2} O}\left(\Delta H_{f 298}^{\circ}\left(H_{2} O\right)+\int_{T_{298}}^{T_{F R}} C_{p}^{\circ}\left(H_{2} O\right) d T\right)=0\end{array}$ \\
\hline $\begin{array}{l}\text { The energy } \\
\text { transported by } \\
\text { solid mass into } \\
\text { FR is divided } \\
\text { into energy } \\
\text { from oxidized } \\
\text { and reduced } \\
\text { fractions of } \\
\text { MeO }\end{array}$ & $\begin{array}{l}\dot{m}_{\text {solid_in }}\left(\Delta H_{f 298}^{\circ}(\text { solid_in })+\int_{T_{298}}^{T_{A R}} C_{p}^{\circ}(\text { solid_in }) d T\right)= \\
\dot{m}_{\text {ox_in }}\left(\Delta H_{f 298}^{\circ}(\text { oxdized form })+\int_{T_{298}}^{T_{A R}} C_{p}^{\circ}(\text { oxdized form }) d T\right) \\
+\dot{m}_{\text {red_in }}\left(\Delta H_{f 298}^{\circ}(\text { reduced form })+\int_{T_{298}}^{T_{A R}} C_{p}^{\circ}(\text { reduced form }) d T\right)\end{array}$ \\
\hline $\begin{array}{l}\text { The energy } \\
\text { transported by } \\
\text { solid mass out } \\
\text { of FR is divided } \\
\text { into energy } \\
\text { from oxidized } \\
\text { and reduced } \\
\text { fractions of } \\
\text { MeO }\end{array}$ & $\begin{array}{l}\dot{m}_{\text {solid_out }}\left(\Delta H_{f 298}^{\circ}(\text { solid_out })+\int_{T_{298}}^{T_{F R}} C_{p}^{\circ}(\text { solid_out }) d T\right) \\
=\dot{m}_{\text {ox_out }}\left(\Delta H_{f 298}^{\circ}(\text { oxdized form })+\int_{T_{298}}^{T_{F R}} C_{p}^{\circ}(\text { oxdized form }) d T\right) \\
+\dot{m}_{\text {red_out }}\left(\Delta H_{f 298}^{\circ}(\text { reduced form })+\int_{T_{298}}^{T_{F R}} C_{p}^{\circ}(\text { reduced form }) d T\right)\end{array}$ \\
\hline
\end{tabular}


Table 3.3: Mass and energy balance for control volume 2 (i.e. Air reactor)

\begin{tabular}{|c|c|}
\hline \multicolumn{2}{|r|}{ Mass Balance } \\
\hline $\begin{array}{l}\text { Total Mass } \\
\text { Balance }\end{array}$ & $\dot{m}_{\text {solid_in }}-\dot{m}_{\text {solid_out }}+\dot{m}_{\text {air }}-\dot{m}_{O_{2}-\text { out }}-\dot{m}_{N_{2}}=0$ \\
\hline $\begin{array}{l}\text { Equation for } \\
\text { solid mass } \\
\text { transported } \\
\text { into the FR }\end{array}$ & $\begin{array}{l}\text { The oxidized and reduced percentage of the solid mass into the FR is given as } \\
\dot{m}_{\text {solid_in }}=\dot{m}_{\text {ox_in }}+\dot{m}_{\text {red_in }}=\frac{X_{A R}}{\left(1-R_{o}+X_{A R} R_{o}\right)} \dot{m}_{\text {solid_in }}+\frac{\left(1-X_{A R}\right)\left(1-R_{o}\right)}{\left(1-R_{o}+X_{A R} R_{o}\right)} \dot{m}_{\text {solid_in }}\end{array}$ \\
\hline $\begin{array}{l}\text { Equation for } \\
\text { solid mass } \\
\text { transported } \\
\text { out of FR }\end{array}$ & $\begin{array}{l}\text { The oxidized and reduced percentage of the solid mass out of the FR is given as } \\
\dot{m}_{\text {solid_out }}=\dot{m}_{\text {ox_out }}+\dot{m}_{\text {red_out }}=\frac{X_{F R}}{\left(1-R_{o}+X_{A R} R_{o}\right)} \dot{m}_{\text {solid_in }}+\frac{\left(1-X_{F R}\right)\left(1-R_{o}\right)}{\left(1-R_{o}+X_{A R} R_{o}\right)} \dot{m}_{\text {solid_in }}\end{array}$ \\
\hline $\begin{array}{l}\text { Equation for } \\
\text { air flow rate }\end{array}$ & $\dot{m}_{\text {Air }}=\frac{\lambda_{\text {air }}}{0.233} M W_{O_{2}} \frac{\dot{m}_{\text {Fuel }}}{M W_{\text {Fuel }}} \frac{v_{b}}{v_{a}}$ \\
\hline $\begin{array}{l}\text { Equation for } \\
\mathrm{O}_{2} \text { and } \mathrm{N}_{2} \\
\text { flow Rates }\end{array}$ & $\dot{m}_{O_{2}-\text { out }}=\left(\lambda_{\text {air }}-1\right) M W_{O_{2}} \frac{\dot{m}_{\text {Fuel }}}{M W_{\text {Fuel }}} \frac{v_{b}}{v_{a}} \quad$ and $\quad \dot{m}_{N_{2}}=\frac{0.767 \lambda_{\text {air }}}{0.233} M W_{O_{2}} \frac{\dot{m}_{\text {Fuel }}}{M W_{\text {Fuel }}} \frac{v_{b}}{v_{a}}$ \\
\hline \multicolumn{2}{|r|}{ Energy Balance } \\
\hline $\begin{array}{l}\text { Total energy } \\
\text { balance }\end{array}$ & $\begin{array}{l}\dot{m}_{\text {solid_in }}\left(\Delta H_{f 298}^{\circ}(\text { solid_in })+\int_{T_{298}}^{T_{A R}} C_{p}^{\circ}(\text { solid_in }) d T\right) \\
-\dot{m}_{\text {solid_out }}\left(\Delta H_{f 298}^{\circ}(\text { solid_out })+\int_{T_{298}}^{T_{F R}} C_{p}^{\circ}(\text { solid_out }) d T\right)+\dot{m}_{\text {air }}\left(\Delta H_{f 298}^{\circ}(\text { air })+\int_{T_{298}}^{T_{F R}} C_{p}^{\circ}(\text { air }) d T\right) \\
-\dot{m}_{N_{2}}\left(\Delta H_{f 298}^{\circ}\left(N_{2}\right)+\int_{T_{298}}^{T_{A R}} C_{p}^{\circ}\left(N_{2}\right) d T\right)-\dot{m}_{O_{2}}\left(\Delta H_{f 298}^{\circ}\left(O_{2}\right)+\int_{T_{298}}^{T_{A R}} C_{p}^{\circ}\left(O_{2}\right) d T\right)=Q\end{array}$ \\
\hline $\begin{array}{l}\text { The energy } \\
\text { transported by } \\
\text { solid mass into } \\
\text { FR is broken } \\
\text { into energy } \\
\text { from oxidized } \\
\text { and reduced } \\
\text { fractions of } \\
\text { Metal oxide }\end{array}$ & $\begin{array}{l}\dot{m}_{\text {solid_in }}\left(\Delta H_{f 298}^{\circ}(\text { solid_in })+\int_{T_{298}}^{T_{A R}} C_{p}^{\circ}(\text { solid_in }) d T\right)= \\
\dot{m}_{\text {ox_in }}\left(\Delta H_{f 298}^{\circ}(\text { oxdized form })+\int_{T_{298}}^{T_{A R}} C_{p}^{\circ}(\text { oxdized form }) d T\right) \\
+\dot{m}_{\text {red_in }}\left(\Delta H_{f 298}^{\circ}(\text { reduced form })+\int_{T_{298}}^{T_{A R}} C_{p}^{\circ}(\text { reduced form }) d T\right)\end{array}$ \\
\hline $\begin{array}{l}\text { The energy } \\
\text { transported by } \\
\text { solid mass out } \\
\text { of FR is broken } \\
\text { into energy } \\
\text { from oxidized } \\
\text { and reduced } \\
\text { fractions of } \\
\text { Metal oxide }\end{array}$ & $\begin{array}{l}\dot{m}_{\text {solid_out }}\left(\Delta H_{f 298}^{\circ}(\text { solid_out })+\int_{T_{298}}^{T_{F R}} C_{p}^{\circ}(\text { solid_out }) d T\right) \\
=\dot{m}_{\text {ox_out }}\left(\Delta H_{f 298}^{\circ}(\text { oxdized form })+\int_{T_{298}}^{T_{F R}} C_{p}^{\circ}(\text { oxdized form }) d T\right) \\
+\dot{m}_{\text {red_out }}\left(\Delta H_{f 298}^{\circ}(\text { reduced form })+\int_{T_{298}}^{T_{F R}} C_{p}^{\circ}(\text { reduced form }) d T\right)\end{array}$ \\
\hline
\end{tabular}




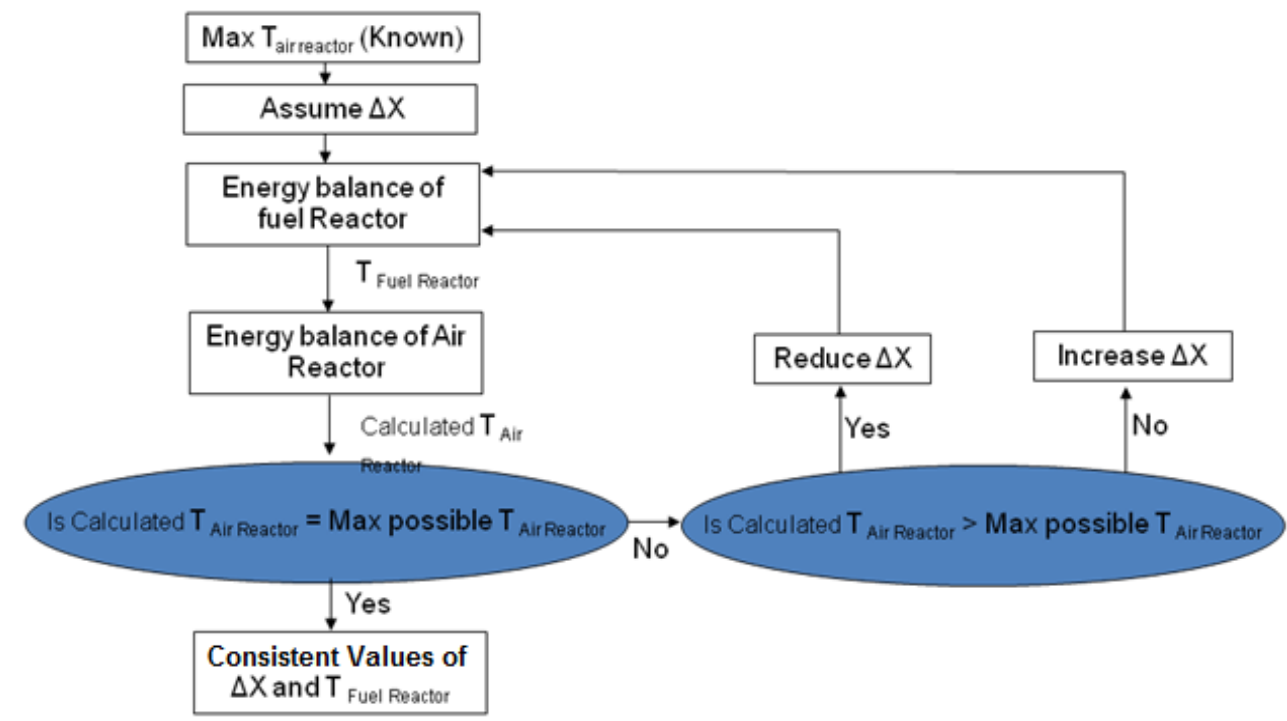

Fig. 3.2: Flow chart for the iterative procedure for calculating CLC system mass and energy balance.

\subsection{Design Calculations for $2 \mathrm{~kW}$ CLC reactor utilizing solid fuels:}

Coal forms a major portion of the fossil fuels used in the United States and is relatively abundantly available. Hence, it is desirable that a CLC system use coal as fuel.

The thermodynamic model developed above, is used for designing a CLC system utilizing coal as fuel and iron oxide as the oxygen carrier. The input values are assumed to be the following:

1) Thermal power required from the $\mathrm{CLC}$ system $=2 \mathrm{KW}$.

2) Maximum temperature allowed in the Air Reactor $=1250 \mathrm{~K}$

3) Fuel is char with calorific value of roughly $32.8 \mathrm{MJ} / \mathrm{kg}$ and the metal oxide particle is assumed to be of 200 micron size with density of $3500 \mathrm{~kg} / \mathrm{m}^{3}$.

The choices of power rating and temperature of operation is based on suitability for an experimental facility being setup at National Energy Technology Lab (NETL).

Coal in general consists of many different chemical elements with usually a significant part being carbon along with lesser quantities of oxygen, hydrogen, sulfur as well as complex minerals and ash. In the present analysis it is assumed that coal consists only of char, treated as pure carbon, thus simplifying the calculations. The following reactions for reduction and oxidation are assumed: 
Char Gasification by $\mathrm{CO}_{2}$

$$
\mathrm{C}+\mathrm{CO}_{2} \rightarrow 2 \mathrm{CO}
$$

Char Gasification by $\mathrm{H}_{2} \mathrm{O}$

$$
\mathrm{C}+\mathrm{H}_{2} \mathrm{O} \rightarrow \mathrm{CO}+\mathrm{H}_{2}
$$

Metal Oxide Reduction

$$
\begin{aligned}
& 3 \mathrm{Fe}_{2} \mathrm{O}_{3}+\mathrm{CO} \rightarrow 2 \mathrm{Fe}_{3} \mathrm{O}_{4}+\mathrm{CO}_{2} \\
& 3 \mathrm{Fe}_{2} \mathrm{O}_{3}+\mathrm{H}_{2} \rightarrow 2 \mathrm{Fe}_{3} \mathrm{O}_{4}+\mathrm{H}_{2} \mathrm{O}
\end{aligned}
$$

For the present thermodynamic calculations the chemical kinetics is ignored and it is assumed that complete fuel oxidation takes place. The net reaction for the FR can then be given as:

$$
6 \mathrm{Fe}_{2} \mathrm{O}_{3}+\mathrm{C} \rightarrow 4 \mathrm{Fe}_{3} \mathrm{O}_{4}+\mathrm{CO}_{2}
$$

The net reaction in the air reactor is given as:

$$
4 \mathrm{Fe}_{3} \mathrm{O}_{4}+\mathrm{O}_{2} \rightarrow 6 \mathrm{Fe}_{2} \mathrm{O}_{3}
$$

The chemical reactions given above are used for the calculations of energy balance in the two reactors.

The output quantities calculated are tabulated in Table 3.4. 
Table 3.4: Output quantities calculated from thermodynamic model.

\begin{tabular}{|l|c|}
\hline 1. Fuel flow rate & $6.7 \times 10^{-5} \mathrm{~kg} / \mathrm{s}$ \\
\hline 2. Air flow rate (Assuming 3 time excess oxygen/air supply) & $1.75 \times 10^{-3} \mathrm{~kg} / \mathrm{s}$ \\
\hline $\begin{array}{l}\text { 3. Steam flow rate (Assuming 3 times of stoichiometric amount needed } \\
\text { for gasification) }\end{array}$ & $3 \times 10^{-4} \mathrm{~kg} / \mathrm{s}$ \\
\hline \begin{tabular}{l} 
4. Cross-sectional area of fuel reactor \\
\hline 5. Cross-sectional area of Air reactor
\end{tabular} & $194.4 \mathrm{~cm}^{2}$ \\
\hline $\begin{array}{l}\text { 6. Superficial velocity of Fuel reactor (Assuming } 5 \text { times minimum } \\
\text { fluidization velocity of metal oxide particles) }\end{array}$ & $0.1 \mathrm{~m} / \mathrm{s}$ \\
\hline $\begin{array}{l}\text { 7. Superficial velocity in Air reactor (Assuming } 3 \text { times terminal velocity } \\
\text { of metal oxide particles) }\end{array}$ & $4.0 \mathrm{~m} / \mathrm{s}$ \\
\hline & \\
8. Metal oxide to metal reduction extent $(\Delta \mathrm{X})$ & 0.1 \\
\hline $\begin{array}{l}\text { 9. Metal oxide inventory required in fuel reactor }(500 \text { times steady state } \\
\text { coal mass) }\end{array}$ & $10 \mathrm{~kg}$ \\
\hline 10. Solid circulation rate & $0.08 \mathrm{~kg} / \mathrm{s}$ \\
\hline 11. Temperature of fuel reactor & $1216.52 \mathrm{~K}$ \\
\hline 12.Temperature of air reactor & $1250 \mathrm{~K}$ \\
\hline
\end{tabular}

Note: It has been assumed that the inlet gases to the FR and AR are at the temperature of those reactors and hence preheating of gas is assumed. Also to maintain the temperature of $A R$ at $1250 \mathrm{~K}$ it has been assumed that $2 \mathrm{~kW}$ of power is being extracted from the AR. The thermodynamic model developed in this section has been used for developing innovative design concepts for the fuel reactor and evaluating them using CFD. The details of this study are provided in chapter 10. 


\section{Chapter 4: CFD Flow Model for Granular Multiphase Flows}

The granular flows can be modeled via computational fluid dynamics (CFD) using either an Eulerian-Lagrangian approach or the Eulerian-Eulerian approach. In the Eulerian-Lagrangian approach the motion of individual particles are tracked. The Eulerian-Eulerian approach models granular flow of the solid particles as a interpenetrating continuum. The ANSYS-FLUENT ${ }^{\mathrm{TM}}$ Eulerian-Eulerian CFD solver was used in the present study.

In the present research work the continuum (Eulerian-Eulerian) approach has been used because of its ability to model infinitely large numbers of particles. Given the large scale of the industrial system that eventually needs to be built, this feature is essential as many billions of particles (on the order of $10^{20}$ to $10^{30}$ ) would have to be modeled. The continuum models have been used for modeling granular flows for more than three decades and have been well documented (Gidaspow, 1992). In the continuum approach each particle variable (such as velocity, temperature, mass, etc.), is averaged over a region that is large in comparison with the particle size. Thus only the bulk behavior of the solids is accounted for. Due to this averaging process, constitutive relationships for viscous stress, heat transfer, etc. are required to complete the model of the particle phase and its interactions with the gas (or liquid) phase.

Granular multiphase flows display complex nonlinear rheologies. Part of the reason for this is because the physics of the granular flow changes considerably depending on the extent of volumetric packing. The granular flows are usually classified into two regimes as follows (Gidaspow, 1992):

1) Kinetic-Collision regime.

2) Frictional regime.

The kinetic regime exists at very low volume fraction $\left(\alpha_{s}<0.05\right)$, where the granular material behaves like a dilute gas. In this regime the mean free path of the grains is large in comparison with the diameter of the grain. At medium concentrations $\left(0.05<\alpha_{s}<0.5\right)$ the kinetic-collisional regime is prevalent. Here there is a continuous interaction between different grain particles due to collisions but the mean free path is still larger than the diameter of the particle. At very large concentrations $\left(\alpha_{s}>0.5\right)$ the frictional regime is prevalent. The particles in this case are closely packed and in constant contact with each other. There are large dissipative frictional forces acting between the particles. 
A good rheological model has to account for all the above regimes accurately. This is difficult as the physics involved in these regimes are considerably different from one other. Also there are considerable overlaps because the dissipation mechanisms prevalent in one regime also are present in the other. In the present chapter, rheological models for the kinetic-collision regime and frictional regimes from the exisiting literature that was used in the present work have been presented, where it has been assumed that the intermediate overlap region is described by the sum of the two models. This assumption appears reasonable so long as the quantitative effects of the kinetic-collision regime tend to zero in the frictional regime and vice-versa.

\subsection{Details of CFD Model:}

The continuity equation for phase $q$ is given as (Ansys Inc. 2006):

$$
\frac{\partial}{\partial t}\left(\varepsilon_{q} \rho_{q}\right)+\nabla \cdot\left(\varepsilon_{q} \rho_{q} \vec{v}_{q}\right)=\sum_{p=1}^{n}\left(\dot{m}_{p q}-\dot{m}_{q p}\right)
$$

where $\varepsilon_{q}$ is the volume fraction of the $q^{\text {th }}$ phase; $\dot{m}_{p q}$ is the mass transfer rate from the $p^{\text {th }}$ to the $q^{\text {th }}$ phase. Each phase consists of a number of species. For example, the gas phase may consist of $\mathrm{CH}_{4}, \mathrm{CO}_{2}$, $\mathrm{H}_{2} \mathrm{O}, \mathrm{N}_{2}$, etc., and the solid phase may consist of $\mathrm{NiO}, \mathrm{Ni}, \mathrm{Fe}_{2} \mathrm{O}_{3}$, etc. A transport equation is solved for each species,

$$
\frac{\partial}{\partial t}\left(\varepsilon_{q} \rho_{q} Y_{i q}\right)+\nabla \cdot\left(\varepsilon_{q} \rho_{q} \vec{v}_{q} Y_{i q}\right)=\sum_{p=1}^{n} \sum_{j=1}^{m}\left(\dot{m}_{i j}{ }^{q p}-\dot{m}_{j i}{ }^{p q}\right)
$$

where $Y_{i q}$ is the mass fraction of species $i$ in the $q^{\text {th }}$ phase and $\dot{m}_{i j}^{q p}$ is the mass transfer rate from the $j^{\text {th }}$ species of the $p^{\text {th }}$ phase to $i^{\text {th }}$ species contained in the $q^{\text {th }}$ phase.

The momentum equation for the gas phase is given as (Ansys Inc., 2006):

$$
\frac{\partial}{\partial t}\left(\varepsilon_{g} \rho_{g} \vec{v}_{g}\right)+\nabla \cdot\left(\varepsilon_{g} \rho_{g} \vec{v}_{g} \vec{v}_{g}\right)=-\varepsilon_{g} \nabla p+\nabla \cdot \bar{\tau}_{g}+\varepsilon_{g} \rho_{g} \vec{g}+\sum_{s=1}^{n}\left(\vec{R}_{s g}+\dot{m}_{s g} \vec{v}_{s g}-\dot{m}_{g s} \vec{v}_{g s}\right)
$$

where $\vec{R}_{s g}=\beta_{s g}\left(\vec{v}_{s}-\vec{v}_{g}\right)$ represents the momentum transfer between the $s^{\text {th }}$ solid phase and the gas phase, the drag term. 
The fluid stress tensor is given as:

$$
\overline{\bar{\tau}}_{g}=\mu_{g} \varepsilon_{g}\left(\nabla \vec{v}_{g}+\nabla \vec{v}_{g}^{T}\right)+\varepsilon_{g} \lambda_{g}\left(\nabla \cdot \vec{v}_{g}\right) \bar{I}
$$

The momentum equation for the $s^{\text {th }}$ solid phase is given as (Ansys Inc., 2006)

$$
\frac{\partial}{\partial t}\left(\varepsilon_{s} \rho_{s} \vec{v}_{s}\right)+\nabla \cdot\left(\varepsilon_{s} \rho_{s} \vec{v}_{s} \vec{v}_{s}\right)=-\varepsilon_{s} \nabla p+\nabla \cdot \bar{\tau}_{s}+\varepsilon_{s} \rho_{s} \vec{g}+\sum_{r=1}^{n}\left(\vec{R}_{r s}+\dot{m}_{r s} \vec{v}_{r s}-\dot{m}_{s r} \vec{v}_{s r}\right)
$$

Here, the subscript, "r" includes all other solid phases, as well as the gas phase.

The fluid-solid and solid-fluid momentum exchange terms are opposite but equal, $\beta_{s g}=-\beta_{g s}$. The model proposed by Gidaspow (1992), a combination of the pressure drop loss suggested by Wen and Yu (1966) (Equation 4.6) and the Ergun equation (Equation 4.7) is used herein. When $\varepsilon_{g}>0.8$ (dilute regions), the fluid-solid exchange coefficient $\beta_{s g}$ is:

$\beta_{s g}=\frac{3}{4} C_{D} \frac{\varepsilon_{s} \varepsilon_{g} \rho_{g}\left|\vec{v}_{s}-\vec{v}_{g}\right|}{d_{s}} \varepsilon_{g}{ }^{-2.65}$, where $C_{D}=\frac{24}{\varepsilon_{g} \operatorname{Re}_{s}}\left[1+0.15\left(\varepsilon_{g} \operatorname{Re}\right)^{0.687}\right]$

When $\varepsilon_{g}<0.8$,

$\beta_{s g}=150 \frac{\varepsilon_{s}\left(1-\varepsilon_{g}\right) \mu_{g}}{\varepsilon_{g} d_{s}^{2}}+1.75 \frac{\rho_{g} \varepsilon_{s}\left|\vec{v}_{s}-\vec{v}_{g}\right|}{d_{s}}$

As discussed earlier the stress tensor $\bar{\tau}_{s}$ has to adequately describe the flow for any of the granular regimes. As suggested by Savage (1984), separate expressions for this stress tensor exist for the different regimes and it has been assumed that the net behavior in general is the sum of values of this stress tensor in all regimes.

\section{Rheological model in the kinetic and collisional regimes:}

The granular solid phase stress tensor, arising from particle-particle collisions, is given as (Ansys Inc., 2006): 


$$
\bar{\tau}_{s}=-p_{s} \overline{\bar{I}}+\mu_{s} \varepsilon_{s}\left(\nabla \vec{v}_{s}+\nabla \vec{v}_{s}^{T}\right)+\varepsilon_{s} \lambda_{s}\left(\nabla \cdot \vec{v}_{s}\right) \overline{\bar{I}}
$$

Here $p_{s}$ is the solid-pressure and $\mu_{s}$ is the granular viscosity.

According to Ogawa et al. (1980), in this regime the particles are in continuous fluctuating and random motion within the fluid. They suggest that the mechanical energy of the particles is first transformed to this random kinetic energy and then subsequently dissipated as thermal heat due to inelastic collisions of granular particles because of the random/chaotic oscillations. This random kinetic energy is produced from mechanical energy due to shear. The internal energy is the energy dissipated as heat.

\section{Mechanical Energy $\rightarrow$ Energy of Random Motion $\rightarrow$ Internal Energy}

The kinetic energy of the particle due to random motion is quite often defined as the granular temperature and is given as:

$\Theta_{s}=\frac{1}{3}\left(\overline{c^{\prime}} \bar{c}^{\prime}\right)$

where,

$c^{\prime}=$ Random fluctuating part of the particle velocity.

The kinetic energy of the random motion per unit mass is given by:

$E_{s}=\frac{1}{2}\left(c^{\prime} c^{\prime}\right)=\frac{3}{2} \Theta_{s}$

The granular temperature or random motion of the particles is the primary cause of momentum transfer between particles and hence determines the solid pressure and solid viscosity of the granular medium. Therefore in order to model the rheological stress tensor in the kinetic and collisional regime it is important to understand the evolution of the granular temperature. As discussed earlier the production of random kinetic energy is due to work done by shear forces acting on the granular medium (Ansys Inc., 2006).

$\tau_{s}:\left(\nabla \vec{u}_{s}\right)=$ Generation of random kinetic energy by solid stress tensor 
The random kinetic energy is dissipated through inelastic granular collisions and due to energy transfer to the fluid.

Generation of random kinetic energy = Dissipation through collision + energy transfer from fluid.

Dissipation through collision $=\gamma_{\Theta m}=\frac{12\left(1-e_{s s}^{2}\right) g_{0, s s}}{d_{s} \sqrt{\pi}} \rho_{s} \varepsilon_{s}^{2} \Theta_{s}^{3 / 2}$

Dissipation in the fluid $=\phi_{l s}=3 \beta_{s} \Theta_{s}$

To calculate the granular temperature equilibrium between production and dissipation of the random kinetic energy is assumed (Ansys Inc., 2006); therefore:

$\tau_{s}:\left(\nabla \vec{u}_{s}\right)=\gamma_{\Theta m}+\phi_{l s}$

The constitutive equations for the kinetic-collisional regime are generally developed from the Boltzmann's theory for dense gases (Lun et al, 1984). The Boltzmann equation deals with the time evolution of the distribution function $f(x, p, t)$ in single-particle phase space, where $x$ and $p$ are position and momentum, respectively. The Boltzmann equation is given as

$\frac{\partial f}{\partial t}+c \frac{\partial f}{\partial r}+F \frac{\partial f}{\partial c}=\left(\frac{\partial f}{\partial t}\right)_{\text {Coll }}$

Using the Boltzmann equation it is possible to deduce a granular stress tensor for the kinetic and collisional regimes (Lun et al, 1984).

The solids stress is based on Lun et al. (1984) and accounts for collision between particles.

$p_{s}=\varepsilon_{s} \rho_{s} \Theta_{s}+2 \rho_{s}\left(1+e_{s s}\right) \varepsilon_{s}^{2} g_{0, s s} \Theta_{s}$

Here $e_{s s}$ is the coefficient of restitution $\left(e_{s s}=0.9\right)$ and $g_{0, s s}$ is the radial distribution function (Ogawa et al., 1980)

$g_{0, s s}=\left[1-\left(\varepsilon_{s} / \varepsilon_{s, \max }\right)^{1 / 3}\right]^{-1}$ 
The solid viscosity is given as (Lun et al., 1984):

$\mu_{s}=\frac{4}{5} \varepsilon_{s} \rho_{s} d_{s} g_{0, s s}\left(1+e_{s s}\right)\left(\frac{\Theta}{\pi}\right)^{1 / 2}+\frac{10 \rho_{s} d_{s} \sqrt{\Theta_{s} \pi}}{96 \varepsilon_{s}\left(1+e_{s s}\right) g_{0, s s}}\left[1+\frac{4}{5} g_{0, s s} \varepsilon_{s}\left(1+e_{s s}\right)\right]^{2}$

and the solids bulk viscosity is (Lun et al., 1984):

$\lambda_{s}=\frac{4}{3} \varepsilon_{s} \rho_{s} d_{s} g_{0, s s}\left(1+e_{s s}\right)\left(\frac{\Theta}{\pi}\right)^{1 / 2}$

\section{Rheological model in the frictional regime:}

When the solid fraction becomes close to the dense packing limit, enduring frictional interactions between particles becomes important. Therefore, for $\alpha_{s}>0.5$, an additional frictional term is added to the solid stress (Schaeffer, 1987):

$\bar{\tau}_{\text {friction }}=-p_{\text {friction }} \overline{\bar{I}}+\mu_{\text {friction }}\left(\nabla \vec{u}_{s}+\nabla \vec{u}_{s}^{T}\right)$,

The frictional pressure is described herein by a functional form as provided by Johnson and Jackson (1987):

$p_{\text {friction }}=\operatorname{Fr} \frac{\left(\varepsilon_{s}-\varepsilon_{s, \min }^{*}\right)^{n}}{\left(\varepsilon_{s, \max }^{*}-\varepsilon_{s}\right)^{p}}$

Suitable constants for the above model have been suggested by numerous researchers: Srivastava and Sunderesan (2003), Ocone et al. (1993). In the present work $F r=5.0, n=2, p=3, \varepsilon_{s, \text { min }}^{*}=0.5$ and $\varepsilon_{s, \max }^{*}=0.63$

To define the viscosity of the frictional granular flow theories developed by Schaeffer, 1987 and Langroudi et al. (2010) have been used. Schaeffer (1987) uses the plasticity theory to describe the dense frictional regime. Langroudi et al. (2010) develop their model heuristically based on the functional form of Schaeffer's viscosity model. To derive the relationship for the viscosity from plasticity theory, two parameters must be defined: 
1) the yield criterion.

2) the flow rule.

The yield criterion establishes a stress space within which the granular medium will behave in a rigid elastic manner. Once the distortional forces are large enough to cause the state of stress to be on or outside the yield surface then the granular material will flow in a viscoplastic manner. In the present case, only the relations for the viscoplastic behavior of the granular material are used, ignoring the elastic region. It is assumed that in fluidized bed applications the distortional forces (such as drag) are always large enough so that the stress state is never within the yield surface.

The flow rule determines the direction of plastic straining and is given as (Schaeffer, 1987):

$$
d \dot{\varepsilon}_{\text {Plastic }}=\lambda \frac{\partial Q}{\partial \sigma}
$$

where $\lambda$ is the plastic multiplier, and

$Q$ is the plastic potential or a representative of the rate of work by the plastic stresses.

A commonly used yield function for the viscoplastic granular flows was suggested by Drucker and Prager (1952). Basically, it suggests that the yield stress is directly proportional to the magnitude of the dilatational or hydrostatic compressional stress. In mathematical terms

$$
\left[\frac{1}{2}\{s\}^{T}\{s\}\right]^{1 / 2}=-\bar{\sigma} \sin (\phi)
$$

where $\{s\}$ is the deviatoric stress and $\bar{\sigma}$ is the compressional hydrostatic stress.

The location of the yield surface in the principal stress space in two-dimensions and three dimensions is shown in Fig. 5.1 and Fig. 5.2 below: 


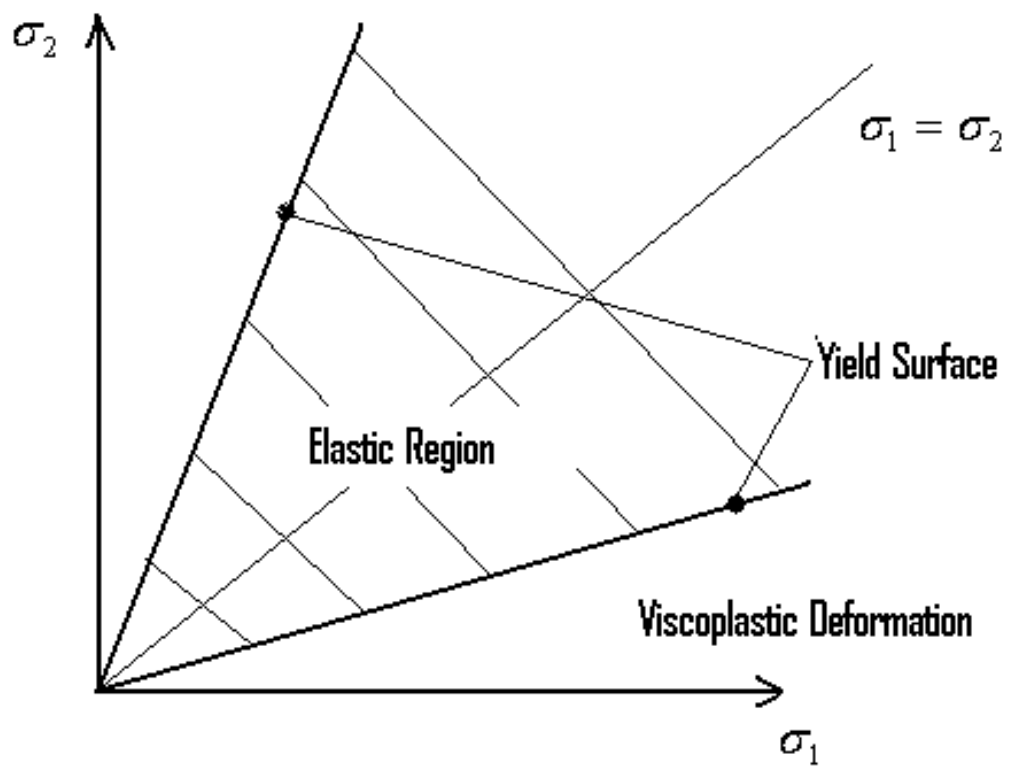

Fig. 4.1: Location of Drucker-Prager (1952) yield surface on two-dimensional principle stress plane.

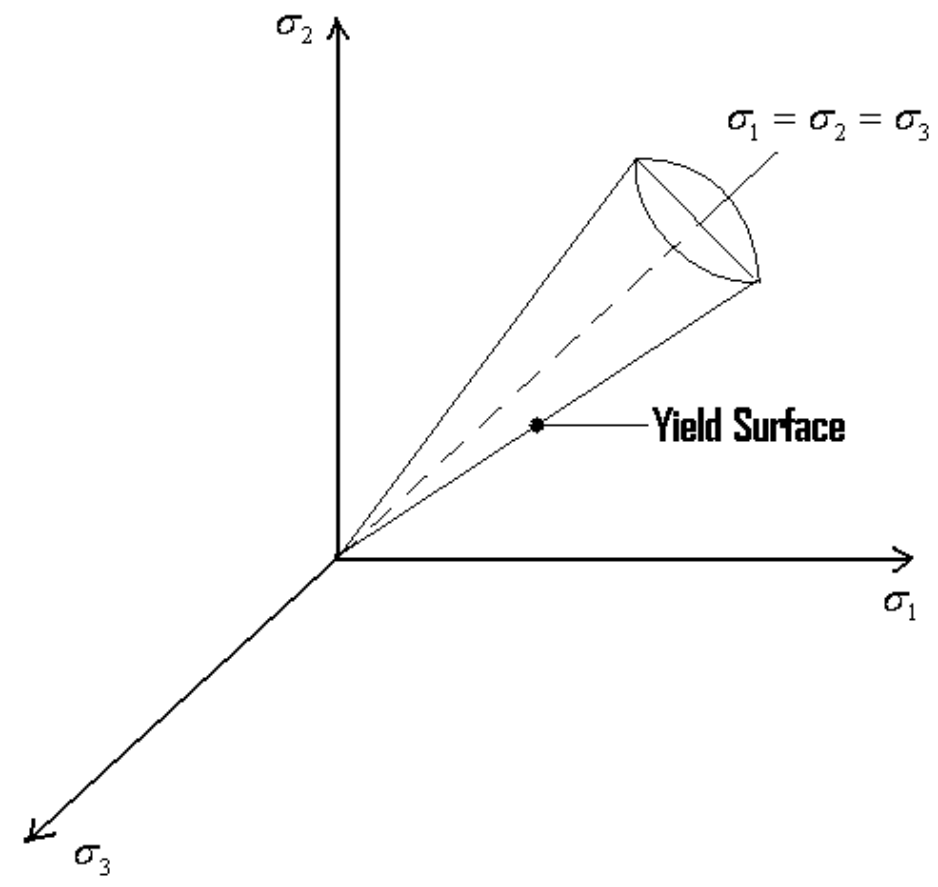

Fig. 4.2: Location of Drucker-Prager (1952) yield surface on three-dimensional principle stress space. 
Darteville (2003) suggested that the Drucker-Prager or modified Von-Mises yield criteria cannot define certain features of granular flows such as dilatation and consolidation. He suggests a good form for the yield function is the Pitman and Schaeffer (1987) yield function given by the following equation:

$\left[\frac{1}{2}\{s\}^{T}\{s\}\right]=\sin ^{2}(\phi)\left\lfloor\left(\bar{\sigma}-P_{\text {friction }}\right)^{2}-P_{\text {friction }}^{2}\right\rfloor$

where $P_{\text {friction }}$ is the frictional pressure as defined by equation 4.22 above.

Figs. 5.3 and 5.4 show the location of the yield surface as defined by the above yield function in two and three dimensional principle stress plane respectively.

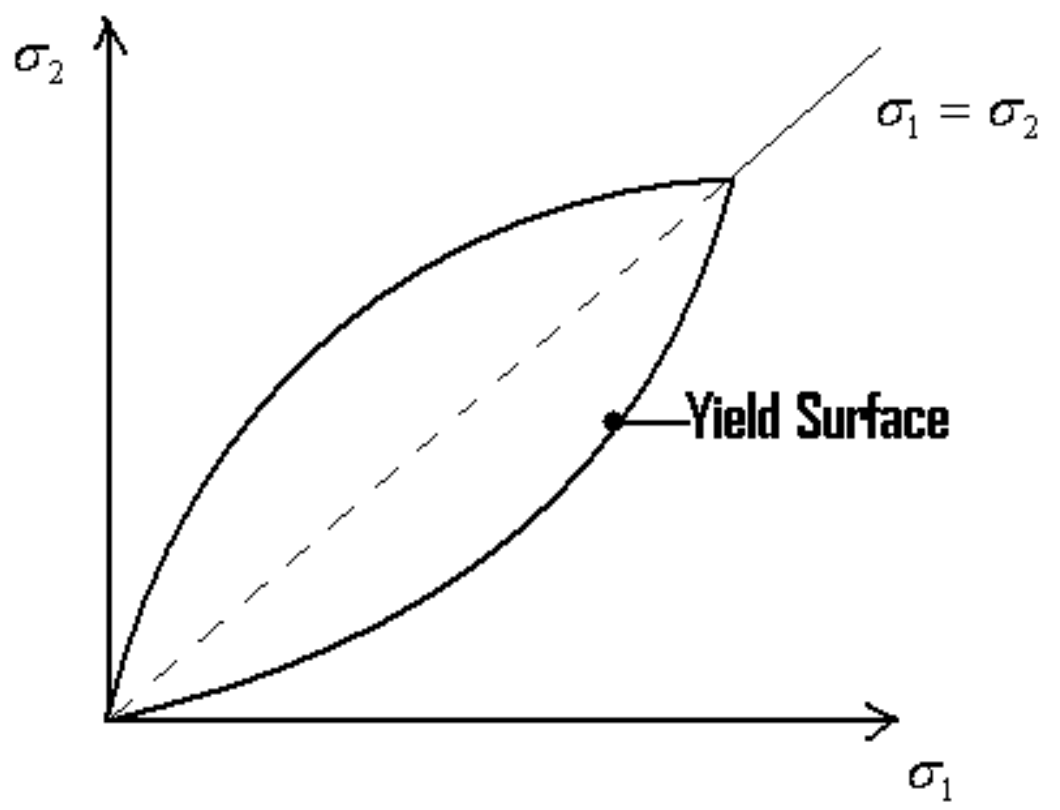

Fig. 4.3: Location of yield surface for Pitman-Schaeffer (1987) model on two-dimensional principle stress plane. 


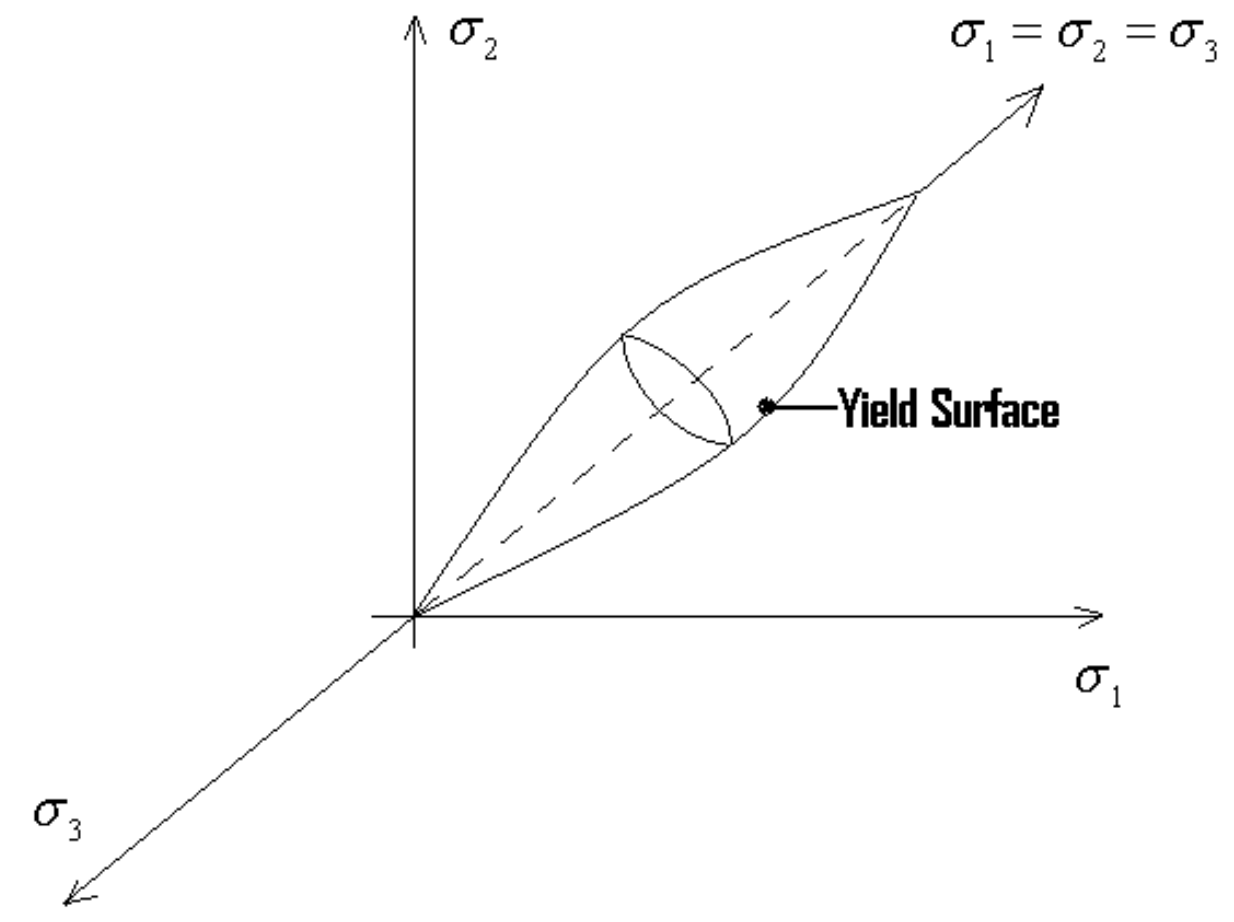

Fig. 4.4: Location of yield surface for Pitman-Schaeffer (1987) model on three-dimensional principle stress space.

By using the Pitman-Schaeffer yield function and by assuming the plastic potential to be the same as the yield function (associated flow rule), an expression for the frictional viscosity can be derived as (Schaeffer, 1987; Darteville, 2003):

$\mu_{\text {friction }}=\frac{P_{\text {friction }} \sin (\phi)}{\sqrt{4 I_{2 D}}}$

where $I_{2 D}$ is the second invariant of the strain tensor.

It is also assumed that the frictional part of the stress becomes significant only when the volume fraction is greater than a critical value.

Based on experimental results, Langroudi et al. (2010) suggest that the frictional viscosity should also be dependent on the rate of strain, according to:

$\mu_{\text {friction }}=a+b|\dot{\gamma}|^{n}$ 
where $\dot{\gamma}$ is the rate of strain. Based on heuristic methods they show that the frictional viscosity can be given as:

$\mu_{\text {friction }}=\sqrt{2} p\left(\frac{\sin \phi+b \cos \phi \times\left(I_{2 D}\right)^{n / 2}}{\sqrt{I_{2 D}}}\right)$

where $b=0.13$ and $n=0.72$. 


\section{Chapter 5: Basic Validation Studies for Granular Flows}

It is important that the CFD models accurately predict both the gas and solids flows in a chemical looping combustion system (CLC). In CLC systems utilizing coal as fuel, there are likely to be particles of various size and density. For example all individual coal particles will vary in size and density as they are consumed. Also, metal oxide particles likely will have a different size than the coal particles. In addition calcium carbonate particles could be used for sulfur capture as is done in several coal combustion units. In general lighter weight particles have a tendency to float on top. This tendency could affect the performance of the reactor. For example if coal particles float on the top of the bed then the combustible gases released by them will not have sufficient contact time to react completely with metal oxide resulting in low combustion efficiencies. Some basic validation studies have been carried out as an initial part of current work to evaluate the accuracy of the CFD model to predict segregation in a reasonable manner.

Apart from segregation it also important that the model be able to predict other important fluid mechanical aspects such as the bubble size, bed height, bubble velocity, etc. Detailed studies on this area have been carried out by several researchers using mathematical models that are either the same or very similar to those used in the present studies (Hulme et al., 2005; Chandrasekaran et al., 2005). Chandrasekaran et al. carried out X-ray fluoroscopy measurements and CFD simulations to characterize the hydrodynamics in a pseudo 2-D gas-solids bubbling fluidized bed using polyethylene resin and glass beads. Bubble properties such as bubble frequency, bubble size, bubble number distribution and bubble diameter distribution, were estimated from the experiments using X-ray images. Numerical and experimental data were then compared and a reasonable match between experiments and numerical simulations was obtained. For example, Fig. 5.1 compares bubble size predicted by numerical simulation with the experimental data (Chandrasekeran et al., 2005). A clear increase in bubble size can be seen as a bubble moves upwards. Fig. 5.2 shows the average vertical bubble velocities as a function of bubble diameter (Chandrasekeran et al., 2005). The simulated bubble velocities are higher than those from experiment but the general trends agreed reasonably well.

To verify the capability of the current CFD model to predict segregation, two different experiments from the literature have been simulated: 
1) Goldschmidt et al. (2003) which uses superficial velocities that are close to the minimum fluidization velocity of the particles and hence is considered to be a 'low-velocity' experiment, and

2) Moritomi et al. (1982) which uses velocities that are considerably larger than the minimum fluidization velocity and hence is considered to be a 'high-velocity' experiment.

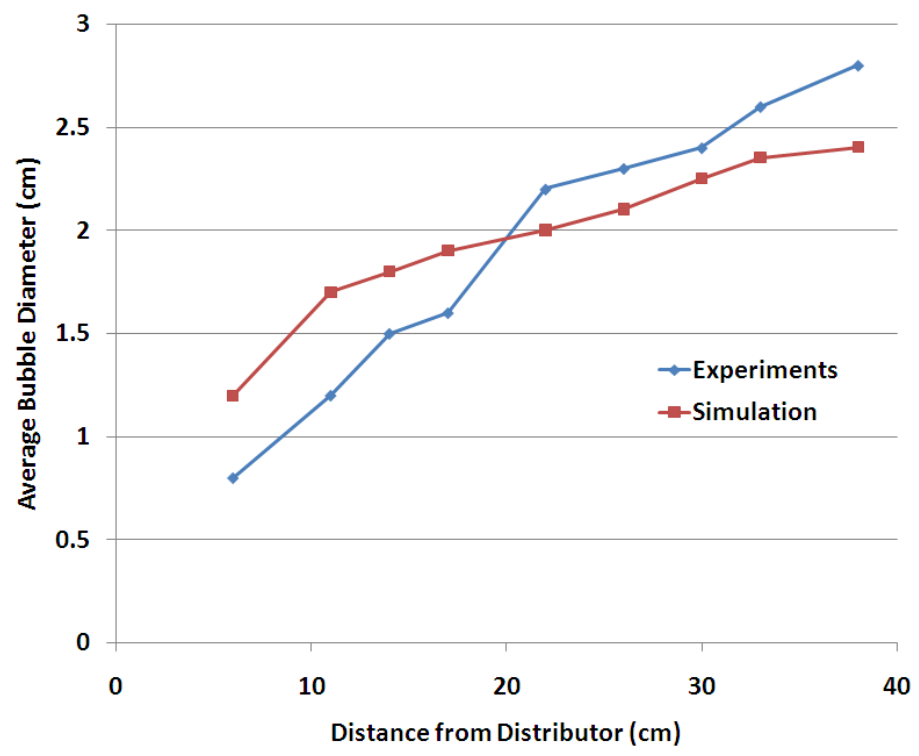

Fig. 5.1: Distance from distributor vs. average bubble diameter (Chandrasekaran et al., 2005).

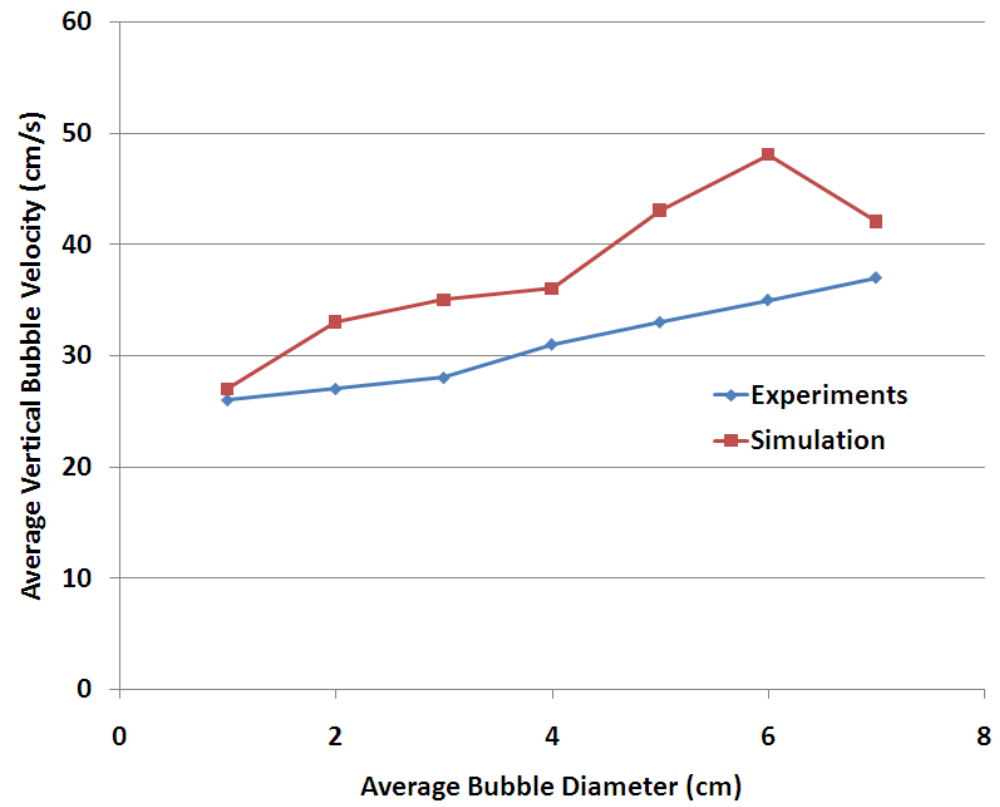

Fig. 5.2: Average bubble diameter vs. average vertical bubble velocity (Chandrasekaran et al., 2005). 


\subsection{Details of Goldschmidt et al. (2003) experimental study}

These simulations (Goldschmidt et al., 2003) have been carried out for a fluidized bed consisting of two particle classes of equal density and different diameter (1.5mm and $2.5 \mathrm{~mm})$. The minimum fluidization velocity of the small particles was $0.78 \mathrm{~m} / \mathrm{s}$ and that of the large particles was $1.25 \mathrm{~m} / \mathrm{s}$. Different superficial velocities were used in the experiments, ranging from $1 \mathrm{~m} / \mathrm{s}$ to $1.6 \mathrm{~m} / \mathrm{s}$. When superficial gas velocities are between the minimum fluidization velocity values of the two particles (i.e. between 0.78 to $1.25 \mathrm{~m} / \mathrm{s}$ ) then segregation occurs. When the velocity is higher than minimum fluidization velocity of the larger particle then mixing occurs. Details of the Goldschmidt experiment are provided in Table 5.1. Table 5.2 provides details about the grid size and numerics used in the simulations.

The comparison of the predicted segregation dynamics with the experiments is shown in Fig. 5.3 for a gas velocity of $1.1 \mathrm{~m} / \mathrm{s}$. The rate of segregation is initially fast but slowly reaches a dynamic equilibrium between mixing and segregation. From Fig. 5.3 it is clear that the intensity of the bed height fluctuations for the small particles is larger in comparison with the large particles and this was clearly captured by the CFD model used. Also, the predicted magnitude of segregation is less in comparison with the experiments. Fig. 5.4 shows the predicted volume fraction of small and large particles in the bed at around 30 seconds of simulation time. The top layer is primarily occupied by smaller particles while the larger particles settle at the bottom. A clear segregation can be observed between the small and large particles. Fig. 5.5 shows the predicted and experimental segregation extent for three different percentages of small particles. In general the extent of segregation is high when the smaller particles are less in concentration. This trend is captured by the simulations, however quantitatively the predicted extent of segregation is less in comparison with experiments. This is not surprising given the numerous modeling assumptions that have been described in chapter 4 to predict the inherent complexities of multiphase flows involving binary mixtures of solid particles. Fig. 5.6 shows the predicted and experimental segregation extent with changes in the bed height. The simulations correctly predict a lower extent of segregation at smaller bed heights. Finally, Fig. 5.7 shows that the CFD model correctly predicts the change in extent of segregation with change in superficial velocity. Thus it can be concluded that present CFD model is able to capture the trends observed in segregation of particles, but quantitatively the predicted segregation might be less than those observed in the experiments. No attempt has been made to improve upon the capabilities of the CFD model to obtain better comparisons 
with experiments as this was considered to be outside the scope of the current research goals. Also, segregation of the coal and metal oxide particles in a CLC FR should be somewhat easier to simulate properly, because of the significant density differences between the coal and metal oxide particles.

Table 5.1: Details of system properties for experiments of Goldschmidt et al. (2003).

\begin{tabular}{|l|r|}
\hline Width of bed (cm) & 15 \\
\hline Height of bed $(\mathrm{cm})$ & 70 \\
\hline Range of inlet velocity (m/s) & 1.0 to 1.6 \\
\hline Total mass of smaller particles (grams) & 247 \\
\hline Total mass of larger particles (grams) & 247 \\
\hline Diameter of smaller particles $(\mathrm{mm})$ & 1.5 \\
\hline Diameter of larger particles $(\mathrm{mm})$ & 2.5 \\
\hline Density of smaller particles $\left(\mathrm{kg} / \mathrm{m}^{3}\right)$ & 2523 \\
\hline Density of larger particles $\left(\mathrm{kg} / \mathrm{m}^{3}\right)$ & 2523 \\
\hline
\end{tabular}

Table 5.2: Details of numerics for simulations of Goldschmidt et al. (2003) experiments.

\begin{tabular}{|l|l|}
\hline Spatial mesh size spacing (figure of mesh) & $50 \times 100(\mathrm{H} \times \mathrm{V})$ Coarse Grid \\
\hline Horizontal grid sizing expansion factor & 1 (Uniform) \\
\hline Vertical grid sizing expansion factor & 1.02 \\
\hline Spatial operators/scheme for each Phase & QUICK \\
\hline $\begin{array}{l}\text { Spatial operators/scheme of other } \\
\text { equations }\end{array}$ & Second Order Upwind \\
\hline Time integration scheme & First Order Implicit \\
\hline Time step size & 0.0001 seconds \\
\hline Iterations per time step & 20 iterations \\
\hline
\end{tabular}




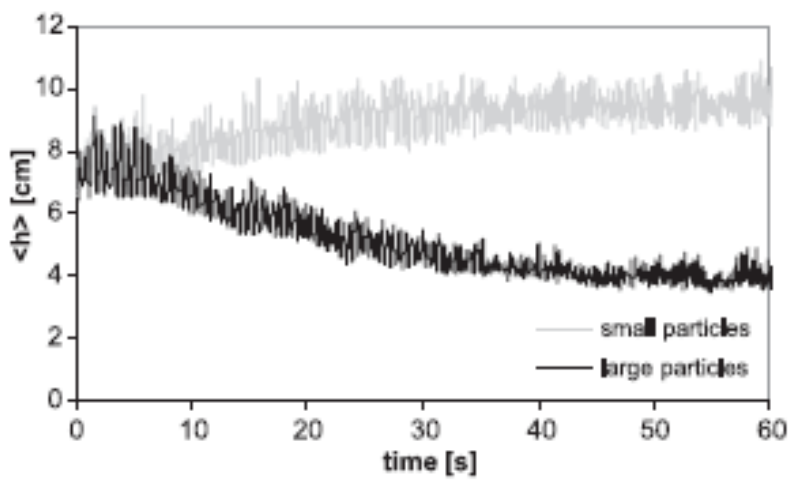

a) Experiments

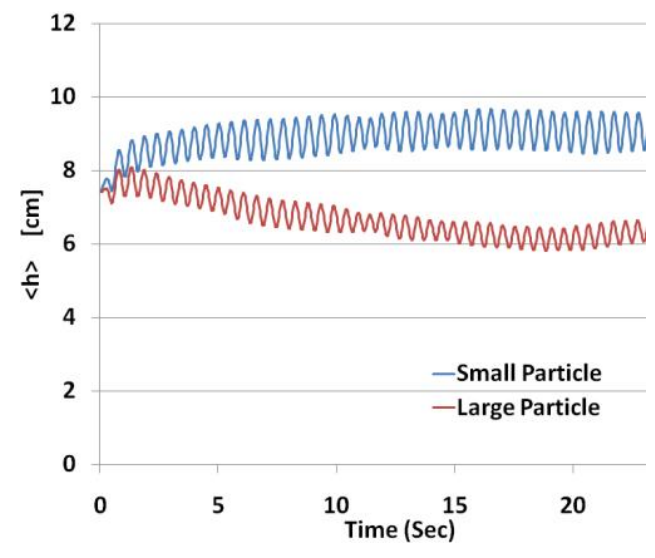

b) Simulations

Fig. 5.3: Average bed heights for small and large particles with time for simulations of Goldschmidt et al. (2003) experiments.

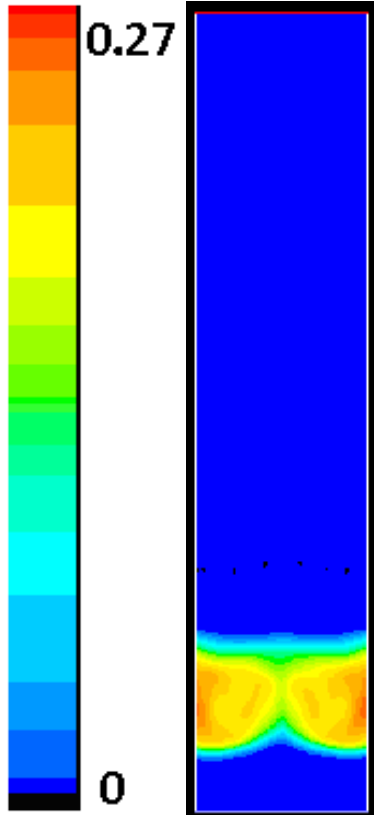

a)Small particles

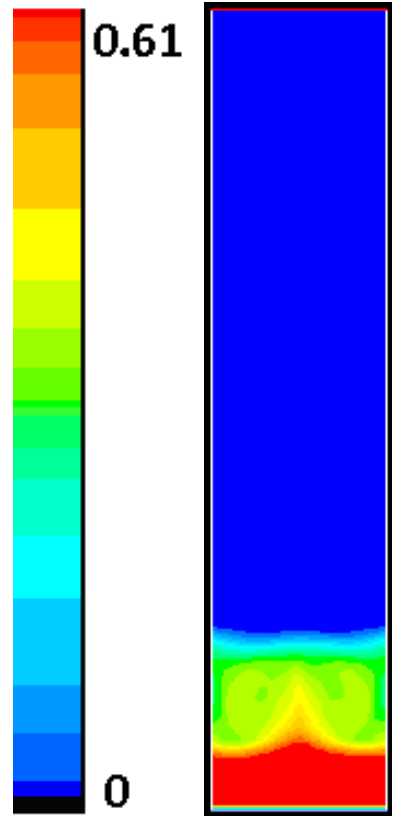

b) Large particles

Fig. 5.4: Particle volume fraction for large and small particles at time=30seconds ( $25 \%$ small particles, superficial velocity $=1.1 \mathrm{~m} / \mathrm{s}$, bed height $=15 \mathrm{~cm}$ ). 


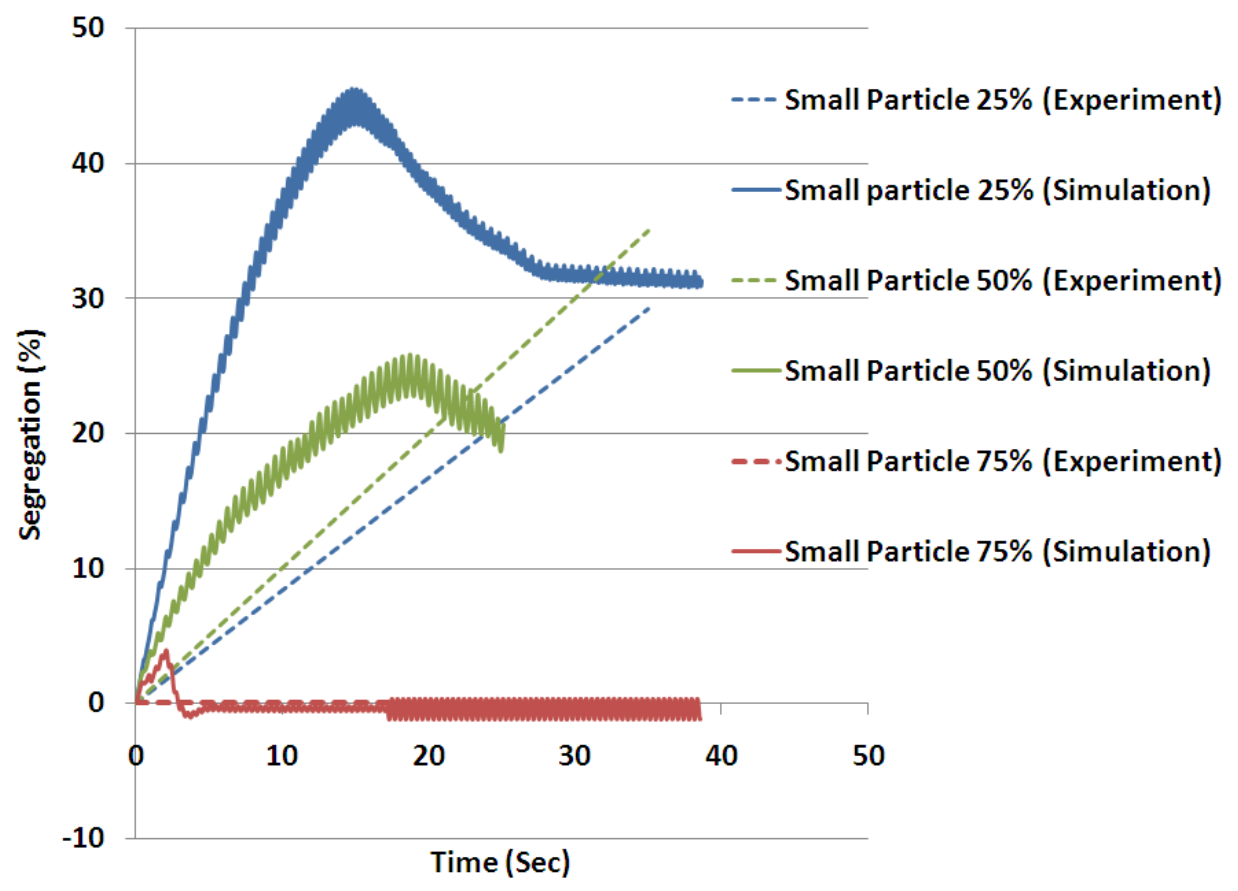

Fig. 5.5: Variation in experimental and predicted segregation extent with time (superficial velocity = $1.1 \mathrm{~m} / \mathrm{s}$ and bed height $=15 \mathrm{~cm}$ ) for various concentrations of small particles.

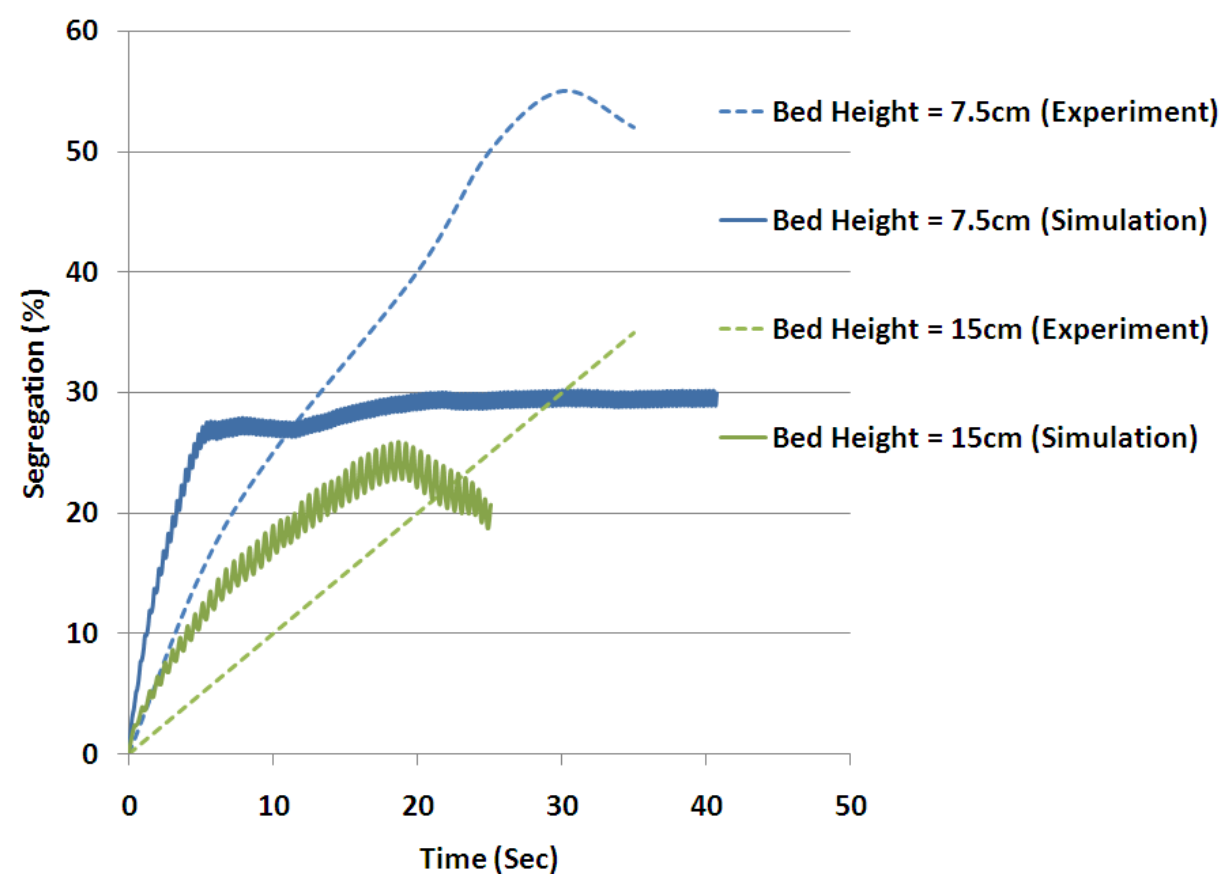

Fig. 5.6: Variation in experimental and predicted segregation extent with time (superficial velocity = $1.1 \mathrm{~m} / \mathrm{s}$ and $50 \%$ small particles) for two different bed heights. 


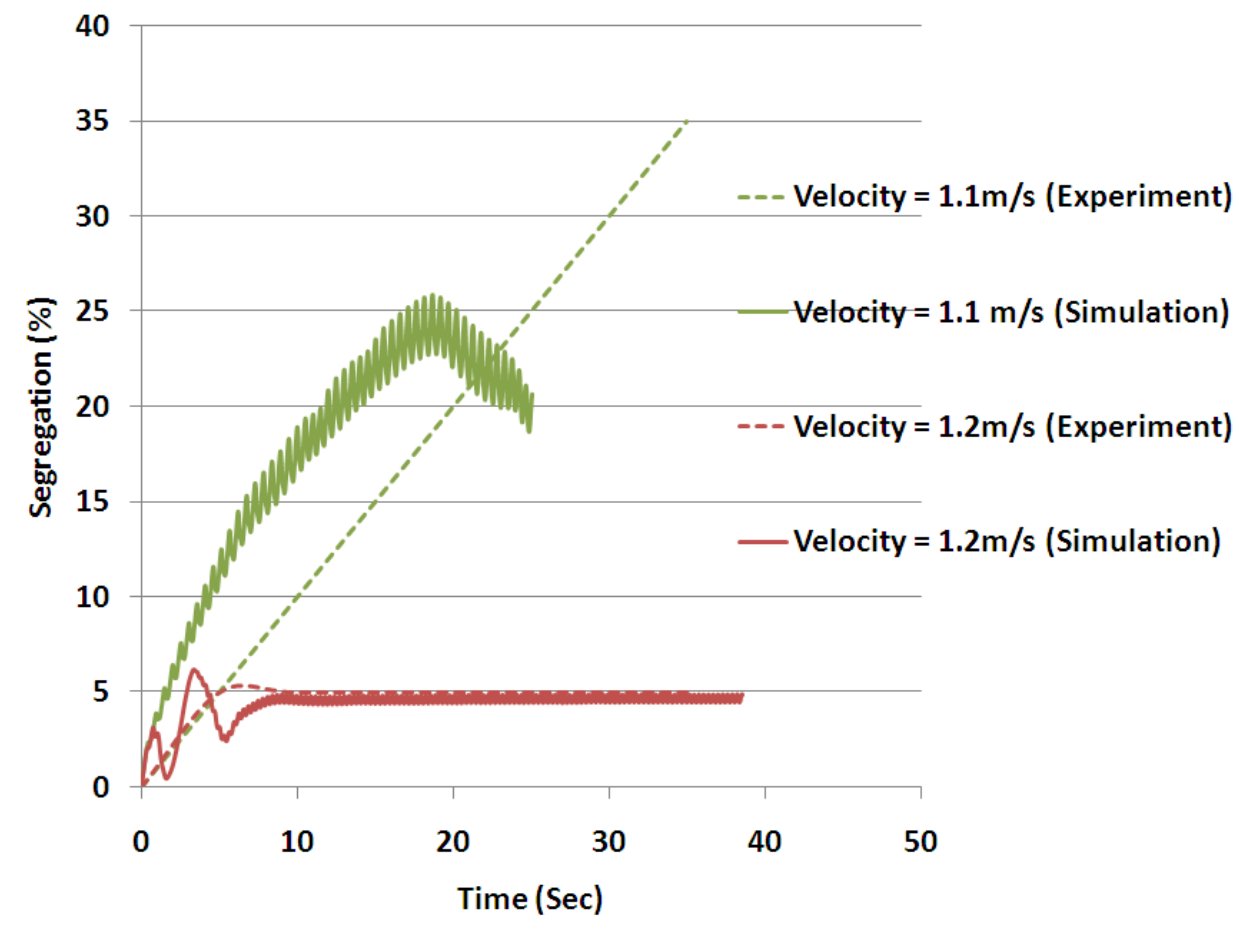

Fig. 5.7: Variation in experimental and predicted segregation extent with time (Bed height $=15 \mathrm{~cm}$ and $\mathbf{5 0 \%}$ small particles) for two different fluidization velocities.

\subsection{Details of Moritomi et al. (1983) experimental study}

The experiments of Moritomi et al. (1983) were carried out for a fluidized bed consisting of two particles, glass beads and hollow char. The glass beads were smaller in size and denser than the char. The bed was fluidized at various velocities ranging from at $2.5 \mathrm{~mm} / \mathrm{s}$ to $12 \mathrm{~mm} / \mathrm{s}$. Table 5.3 provides the details of various parameters used in the Moritomi experiments. Table 5.4 provides the details of the numerics used in the CFD simulations. It was observed that at velocities below $8 \mathrm{~mm} / \mathrm{s}$, the hollow char floated on top of the bed. At a velocity around $8 \mathrm{~mm} / \mathrm{s}$ the bed was fully mixed and no distinct separation between the solid phases was observed. When the velocity was increased beyond $8 \mathrm{~mm} / \mathrm{s}$ the hollow char settled at the bottom and the glass beads started to float on top (Fig. 5.8). This behavior is typical of the inversion process. Very similar quantitative predictions for the same experiments have been shown by Syamlal and O'Brien (1988). The primary reason for the significantly larger bed height predicted by the numerical model (Fig. 5.8) is attributed to the drag law used in the present simulations. The drag experienced by small particles will get affected by the presence of larger particles in the vicinity 
and vice-versa. No attempt has been made to evaluate other drag models since this was outside the scope of the current research project.

Table 5.3: System properties for Moritomi et al. (1983) experiments.

\begin{tabular}{|l|r|}
\hline Width of bed $(\mathrm{cm})$ & 5 \\
\hline Height of bed $(\mathrm{cm})$ & 30 \\
\hline Range of inlet velocity (cm/s) & 100 \\
\hline Total mass of glass beads (grams) & 50 \\
\hline Total mass of hollow char (grams) & 0.163 \\
\hline Diameter of glass beads $(\mathrm{mm})$ & 0.775 \\
\hline Diameter of hollow char $(\mathrm{mm})$ & 2450 \\
\hline Density of glass beads $\left(\mathrm{kg} / \mathrm{m}^{3}\right)$ & 1500 \\
\hline Density of hollow char $\left(\mathrm{kg} / \mathrm{m}^{3}\right)$ & \\
\hline
\end{tabular}

Table 5.4: Numerics used for simulations of Moritomi et al. (1983) experiments.

\begin{tabular}{|l|l|}
\hline Spatial mesh size spacing (figure of mesh) & $50 \times 100(\mathrm{H} \times \mathrm{V})$ \\
\hline Horizontal grid sizing expansion factor & 1 (Uniform) \\
\hline Vertical grid sizing expansion factor & 1.02 \\
\hline Spatial operators/scheme for each phase & QUICK \\
\hline $\begin{array}{l}\text { Spatial operators/scheme of other } \\
\text { equations }\end{array}$ & $\begin{array}{l}\text { Second Order } \\
\text { Upwind }\end{array}$ \\
\hline Time integration scheme & First Order Implicit \\
\hline Time step size & 0.01 seconds \\
\hline Iterations per time step & 30 iterations \\
\hline
\end{tabular}

Simulations were carried out at five superficial velocities $2.5 \mathrm{~mm} / \mathrm{s}, 5.5 \mathrm{~mm} / \mathrm{s}, 7.5 \mathrm{~mm} / \mathrm{s}, 9.5 \mathrm{~mm} / \mathrm{s}$ and $11.5 \mathrm{~mm} / \mathrm{s}$. Fig. 5.8 shows the plot for interface locations of the two solid phases as observed in experiments and as predicted by the simulations. The segregation of solids observed in the Moritomi et al. (1983) experiments can be qualitatively captured by the present CFD model.

40 


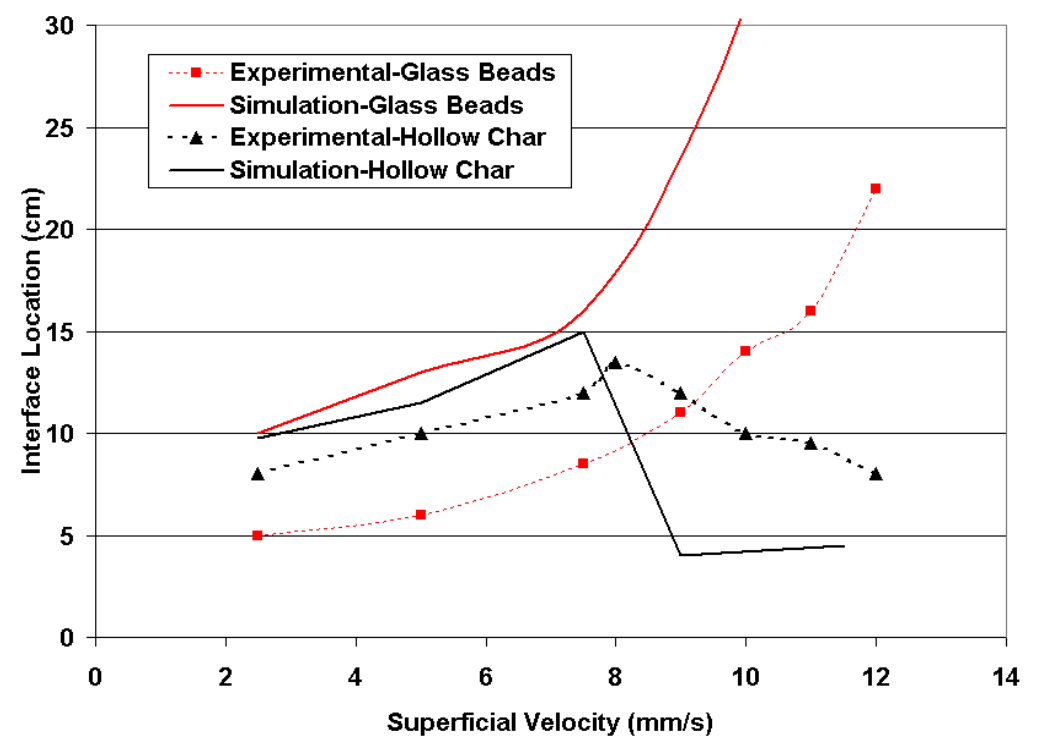

Fig. 5.8: Comparison of experimental and predicted Interface location of glass beads and hollow char.

\subsection{Conclusion:}

It is observed that a reasonable prediction of bubble size and velocity can be obtained via the CFD model. It is also observed that the trends in segregation of particles at low velocity (close to minimum fluidization as in Goldschmidt et al., 2003) are captured by the CFD model. Also, the segregation at high velocity (several multiples of minimum fluidization as in Moritomi et al., 1983) can also be predicted qualitatively by the CFD model. 


\section{Chapter 6: Chemistry Modeling for CFD}

\section{Introduction:}

Any CLC system using a solid fuel and metal oxide carrier involves both homogeneous and heterogeneous reactions. The homogeneous reaction rates are assumed to be dependent on the concentrations of reacting species. Also, the rate constant of homogeneous reactions is assumed to be dependent on Arrhenius type equations. To model the heterogeneous reactions either the Shrinking Core (SC) or the Grain Model (GM) is used. Both SC and GM are described in detail in Szekely et al. (1976) and Levenspiel (1962). In this chapter the general formulation used for homogeneous and heterogeneous chemistry is presented (for the metal oxide gas reaction as well as char gasification reactions).

\subsection{Details of Homogeneous Reaction Models}

To model the homogeneous reactions Arrhenius expressions are used, ignoring the effects of turbulent fluctuations. Since the reaction rates are generally quite slow, and also the reactor temperatures are relatively low, ignoring the turbulence fluctuations may not be very critical.

Consider the following homogeneous reaction between two gases

$v_{A} A(g)+v_{B} B(g) \rightarrow v_{C} C(g)+v_{D} D(g)$

For a reversible reaction, the molar rate of creation/destruction $\left(\mathrm{kmol} / \mathrm{m}^{3} / \mathrm{s}\right)$ is modeled by

Rate $=k_{f}[A]^{n_{A}}[B]^{n_{B}}-k_{b}[C]^{n_{C}}[D]^{n_{D}}$

$[A]$ is the molar concentration of species $\mathrm{A}$ and similarly for other species.

$\mathrm{n}_{\mathrm{A}}=$ rate exponent for reactant species $A$ and similarly for other species.

The forward rate is given as:

$k_{f}=A T^{\beta} e^{-E / R T}$

The backward rate is calculated from the forward rate by using the equilibrium constant, $K_{E}$

$k_{b}=\frac{k_{f}}{K_{E}}$ 
The rate of consumption of species $A\left(\mathrm{kmol} / \mathrm{m}^{3} / \mathrm{s}\right)$ is given as

$$
\frac{d[A]}{d t}=v_{A}\left(k_{f}[A]^{n_{A}}[B]^{n_{B}}-k_{b}[C]^{n_{C}}[D]^{n_{D}}\right)
$$

The mass rate of consumption of species $A\left(\mathrm{~kg} / \mathrm{m}^{3} / \mathrm{s}\right)$ is given as

$$
\frac{d m_{A}}{d t}=M W_{A} v_{A}\left(k_{f}[A]^{n_{A}}[B]^{n_{B}}-k_{b}[C]^{n_{C}}[D]^{n_{D}}\right)
$$

Similarly, the mass rate of production of species $\mathrm{C}\left(\mathrm{kg} / \mathrm{m}^{3} / \mathrm{s}\right)$ is given as

$$
\frac{d m_{C}}{d t}=M W_{C} v_{C}\left(k_{f}[A]^{n_{A}}[B]^{n_{B}}-k_{b}[C]^{n_{C}}[D]^{n_{D}}\right)
$$

The forward and backward rates as well as the rate exponents are determined from available published literature.

\subsection{Details of Heterogeneous Reaction Models}

The shrinking core (SC) model and the grain model (GM) are two of the most extensively used models by researchers due to their inherent simplicity and capability to represent the reactions in an adequate manner. The SC and GM are conceptually quite different from one another but turn out to be the same in terms of mathematical formulation. A vast number of particle reactions are represented by either shrinking core (SC) model or the changing grain size model (GM). The SC model is for non-porous particles whereas the GM is used for porous particles.

The mathematical form of each model may be derived by looking at the general form of a heterogeneous reaction

$$
v_{A} A(g)+v_{B} B(s) \rightarrow v_{C} C(g)+v_{D} D(s)
$$

Here, $\mathrm{A}$ is a gaseous reactant species and $\mathrm{B}$ is a reactant in the solid particle (e.g. carbon or char). $\mathrm{C}$ is a gaseous product species and $\mathrm{D}$ is the product (e.g., ash) that will remain on the solid particle. The specific details of SC and GM models are discussed below.

\subsubsection{Shrinking Core (SC) Model for Non-Porous Particles}

In the SC model it is assumed that the particle (and the unreacted core) is initially non-porous and the reaction takes place at the receding surface. After the surface reaction has taken place, a porous 
product layer is formed, surrounding the unreacted core, which allows the reactant gas to pass through it and react with unreacted core of the solid particle (See Fig. 6.1).

Different processes, involved in the reaction are:

- Diffusion of gaseous reactant $A$ inwards through the particle-gas boundary layer.

- Diffusion of reactant $A$ through the porous (ash) layer of the particle.

- Reaction of A with 'unreacted' solid surface. This can be further broken down into the following steps:

a. Surface adsorption of $A$ on the solid surface.

b. Reactions involving surface complexes.

c. Desorption of product $C$ from the surface of solid.

- Outward diffusion of product $\mathrm{C}$ through the porous (ash) layer to reach the outer surface of the solid.

- Diffusion of product C outwards through the particle boundary layer.

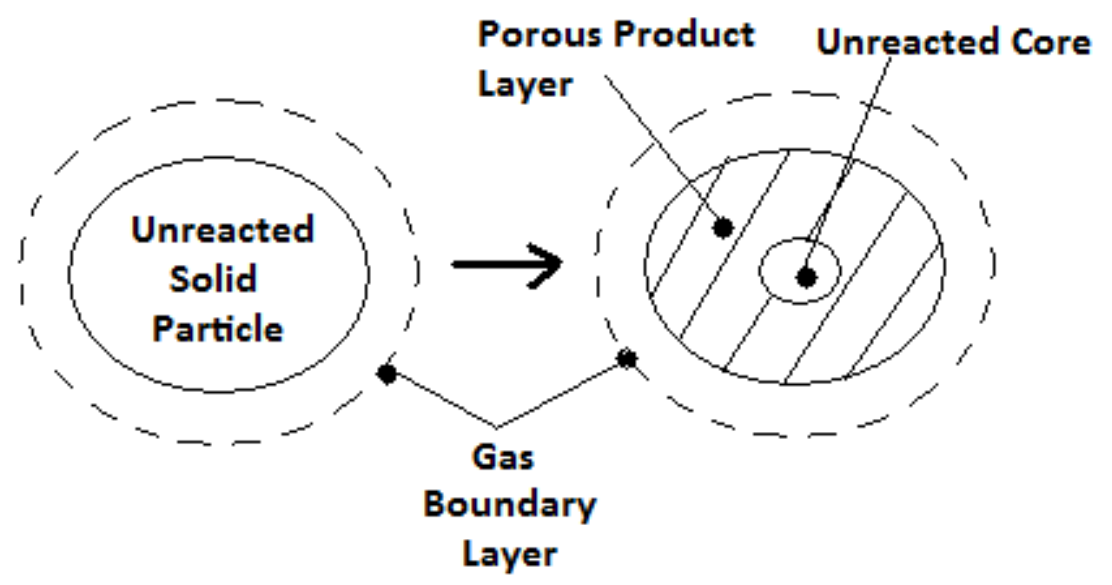

Fig. 6.1: Schematic representation of the Shrinking Core Model for non-porous particles (Szekely et al., 1976).

In many practical applications only one or two of the "reaction-resistances" mentioned above are actually significant, or assumed to be significant. Some examples are:

1) In coal combustion at high temperatures $(T>1700 \mathrm{~K})$ the boundary layer gas diffusion is the major resistance. The resistance due to pore diffusion and chemical reactions is negligible. 
2) For moderate temperature coal combustion ( $1400 \mathrm{~K}<\mathrm{T}<1700 \mathrm{~K})$, the pore diffusion is important and the resistance due to gas boundary layer diffusion can be neglected.

3) In fluidized bed coal gasification applications $(T<1300 \mathrm{~K})$, the reactions are slow enough that only resistance due to chemical reaction is important. The diffusion through boundary layer and through pores is relatively fast.

Now the equations for each of the resistances acting alone are derived, followed by all the resistances acting all at once.

\subsubsection{Boundary layer gas diffusion controlled rate for shrinking core model (i.e. diffusion rate of $A$ through gas film):}

The boundary layer gas diffusion can be expressed by the following reaction (Levenspiel, 1962):

$\frac{1}{4 \pi R^{2}} \frac{d n_{B}}{d t}=b k_{g}\left(C_{A}-\frac{C_{C}}{K_{E}}\right)$

where

$b$ is the stoichiometric coefficient of solid species ' $B$ ' in Equation (6.5).

$n_{B}$ is the number of moles of $\mathrm{B}$ consumed per unit outer surface area of the particle per unit time.

$C_{A}$ is the concentration of reactant $\mathrm{A}$ in free stream and $C_{C}=$ Concentration of product $\mathrm{C}$

$K_{E}$ is the Equilibrium constant to account for the backward rate.

$\mathrm{R}$ is the outer surface radius of the particle for shrinking core model and outer radius of the grain for the grain model.

$k_{g}$ is the diffusion coefficient $(\mathrm{m} / \mathrm{s})$

To obtaining a rate of consumption of solids $\left(\mathrm{kmol} / \mathrm{m}^{3} / \mathrm{s}\right)$ the following steps are carried out.

Now, consider that there are ' $n$ ' particles in per unit volume.

Rate of consumption of solid ' $\mathrm{B}$ ' $\left(\mathrm{kmol} /\left(\mathrm{m}^{3} \mathrm{~s}\right)\right)$ is given as: 
$\dot{N}_{B}=b k_{g}\left(C_{A}-\frac{C_{C}}{K_{E}}\right) n 4 \pi R^{2}$

where $N_{B}=n \times n_{b}$ is the number of moles of ' $\mathrm{B}$ ' $\left(\mathrm{kmol} /\left(\mathrm{m}^{3} \mathrm{~s}\right)\right)$ consumed per $\mathrm{m}^{3}$ volume.

$\dot{N}_{B}=3 b k_{g}\left(C_{A}-\frac{C_{C}}{K_{E}}\right) n \frac{4}{3} \pi R^{2}=\frac{3 b k_{g}}{R}\left(C_{A}-\frac{C_{C}}{K_{E}}\right) \varepsilon_{s}$

The mass rate of consumption of solid ' $\mathrm{B}$ ' $\left(\mathrm{kg} / \mathrm{m}^{3} / \mathrm{s}\right)$ is given as:

$\dot{m}_{B}=M W_{B} \frac{3 b k_{g}}{R}\left(C_{A}-\frac{C_{C}}{K_{E}}\right) \varepsilon_{s}$

Similarly reaction rates for other reacting species $\left(\dot{m}_{A}, \dot{m}_{C}\right.$ and $\left.\dot{m}_{D}\right)$ can be found by accounting for the corresponding stoichiometric coefficients (see Equation 6.5). These reaction rates are applied as source terms in species transport equation (Equation 4.2).

\subsubsection{Diffusion through ash layer (Shrinking core model):}

The diffusion can be expressed by the equation below (Levenspiel, 1962):

$\frac{1}{4 \pi r^{2}} \frac{d n_{B}}{d t}=b D_{e} \frac{d C_{A}}{d r}=$ constant

Here $D_{e}$ is the diffusion coefficient with units of $\mathrm{m}^{2} / \mathrm{s}$.

Integrating with respect to radius ' $r$ ' of the particle yields (Levenspiel, 1962):

$\frac{1}{4 \pi R^{2}} \frac{d n_{B}}{d t}=\frac{b D_{e}}{R^{2}\left(\frac{1}{r_{c}}-\frac{1}{R}\right)}\left(C_{A}-\frac{C_{C}}{K_{E}}\right)$

where $r_{c}$ is the radius of the shrinking core. 
Now consider that there are ' $\mathrm{n}$ ' particles in a computational cell. As above, $N_{B}$ is the number of moles of 'B' consumed per unit volume. Where $N_{B}=n \times n_{b}$.

Rate of consumption of solid ' $\mathrm{B}$ ' $\left(\mathrm{kmol} /\left(\mathrm{m}^{3} \mathrm{~s}\right)\right)$ is given as

$$
\dot{N}_{B}=\frac{3 b D_{e}}{R^{3}\left(\frac{1}{r_{c}}-\frac{1}{R}\right)}\left(C_{A}-\frac{C_{C}}{K_{E}}\right) \varepsilon_{s}
$$

Rearranging yields

Reaction Rate $\left(\mathrm{kmol} / \mathrm{m}^{3} / \mathrm{s}\right)=\dot{N}_{B}=\frac{3 b D_{e}}{R^{2}\left(\frac{R}{r_{c}}-1\right)}\left(C_{A}-\frac{C_{C}}{K_{E}}\right) \varepsilon_{s}$

Now, $X=\frac{m-m_{r e d}}{m_{o x}-m_{r e d}}=\frac{m_{B}}{m_{o x}}=1-\left(\frac{r_{c}}{R}\right)^{3}$

So that

Reaction Rate $\left(\mathrm{kmol} / \mathrm{m}^{3} / \mathrm{s}\right)=\dot{N}_{B}=\frac{3 b D_{e}}{R^{2}\left(\left(\frac{1}{1-X}\right)^{1 / 3}-1\right)}\left(C_{A}-\frac{C_{C}}{K_{E}}\right) \varepsilon_{s}$

$\mathrm{X}$ can be expressed as

$$
X=\frac{m_{B}}{m_{o x}}=\frac{\rho_{s} \varepsilon_{s} Y_{B}}{\rho_{s} \varepsilon_{s}\left(Y_{B}+Y_{D} \frac{M W_{B}}{M W_{D}}\right)}=\frac{Y_{B}}{\left(Y_{B}+Y_{D} \frac{M W_{B}}{M W_{D}}\right)}
$$

The mass rate of consumption of solid ' $\mathrm{B}$ ' $\left(\mathrm{kg} / \mathrm{m}^{3} / \mathrm{s}\right)$ is given as

$$
\dot{m}_{B}=M W_{B} \frac{3 b D_{e}}{R^{2}\left(\left(\frac{1}{1-X}\right)^{1 / 3}-1\right)}\left(C_{A}-\frac{C_{C}}{K_{E}}\right) \varepsilon_{s}
$$


Similarly reaction rates for other reacting species $\left(\dot{m}_{A}, \dot{m}_{C}\right.$ and $\left.\dot{m}_{D}\right)$ can be found by accounting for the corresponding stoichiometric coefficients (see equation 6.5). These reaction rates are applied as source terms in the species transport equation (equation 4.2).

\subsubsection{Chemical Reaction control (Shrinking Core Model):}

The equation is given as (Levenspiel, 1962):

$\frac{1}{4 \pi r_{c}^{2}} \frac{d n_{B}}{d t}=b k_{s}\left(C_{A}-\frac{C_{C}}{K_{E}}\right)$

which can be rewritten in the form given below:

$\frac{1}{4 \pi R^{2}} \frac{d n_{B}}{d t}=b k_{s} \frac{r_{c}^{2}}{R^{2}}\left(C_{A}-\frac{C_{C}}{K_{E}}\right)$

Rate of consumption of solid ' $\mathrm{B}$ ' $\left(\mathrm{kmol} /\left(\mathrm{m}^{3} \mathrm{~s}\right)\right)$ is given as:

$\dot{N}_{B}=\frac{3 b k_{s}}{R} \frac{r_{c}^{2}}{R^{2}}\left(C_{A}-\frac{C_{C}}{K_{E}}\right) \varepsilon_{s}=\frac{3 b k_{s}}{R} \varepsilon_{s}\left(C_{A}-\frac{C_{C}}{K_{E}}\right)(1-X)^{2 / 3}$

\subsubsection{Accounting for all resistances simultaneously:}

To account for resistances due to all sources, the following equation is applicable (Levenspiel, 1962):

$\frac{1}{4 \pi R^{2}} \frac{d n_{B}}{d t}=\frac{b\left(C_{A}-\frac{C_{C}}{K_{E}}\right)}{\frac{1}{k_{g}}+\frac{R^{2}}{D_{e}}\left(\frac{1}{r_{c}}-\frac{1}{R}\right)+\frac{R^{2}}{k_{s} r_{c}^{2}}}$

Let there be ' $\mathrm{n}$ ' particles, so that the rate $\left(\mathrm{kmol} /\left(\mathrm{m}^{3} \mathrm{~s}\right)\right.$ will then be:

$\dot{N}_{B}=\frac{3 b\left(C_{A}-\frac{C_{C}}{K_{E}}\right) \varepsilon_{s}}{\frac{R}{k_{g}}+\frac{R^{2}}{D_{e}}\left(\frac{R}{r_{c}}-1\right)+\frac{R^{3}}{k_{s} r_{c}{ }^{2}}}=\frac{3 b\left(C_{A}-\frac{C_{C}}{K_{E}}\right) \varepsilon_{s}}{\frac{R}{k_{g}}+\frac{R^{2}}{D_{e}}\left(\left(\frac{1}{1-X}\right)^{1 / 3}-1\right)+\frac{R}{k_{s}(1-X)^{2 / 3}}}$ 


\subsubsection{Grain Model (GM) for porous particles}

The GM assumes that the particles are porous and consist of fine grains of solid. These grains can have different shapes (spherical, cylindrical, disc etc). Fig. 6.2 shows a spherical particle with many thousands of smaller size spherical "grains" inside this particle. There are void spaces between the grains as shown in the figure which makes the particle porous. If the particle is sufficiently porous then the diffusion of gas through the pores of the particle will be very fast and the chemical reaction taking place at the surface of the grain will be the limiting process. Therefore the concentration of reacting gas inside the particle in this limit will be equal to the free stream concentration and each of the grains can now be treated as a single solid particle with a radius equal to the grain radius. Therefore each of the small solid grains within the porous particle can now be treated with the shrinking core model.

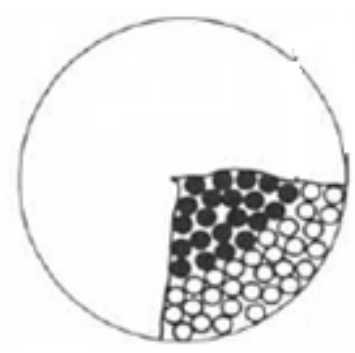

Fig. 6.2: Schematic representation of a porous, spherical particle with spherical grains (Szekely et al., 1976). Dark grains indicate the unreacted core and lighter colored grains indicate reacted outer core.

Different processes involved in the reaction are:

1) Diffusion of gaseous reactant $A$ inwards through the particle boundary layer.

2) Diffusion of reactant $A$ through the pores of the particle.

3) Reaction of $A$ with grain surfaces. This can be further broken down into the following steps:

a. Surface adsorption of $A$ on the grain surface of the solid.

b. Complex reactions involving surface complexes.

c. Desorption of product C from the surface of solid.

4) Outward diffusion of product $C$ through the pores to reach the outer surface of the solid.

5) Diffusion of product C outwards through the particle boundary layer.

Thus it can be seen from above discussion that both SC and GM involve a succession of similar steps. The only difference is that the ash layer diffusion in SC is replaced by the pore diffusion in the GM. It is observed that the form of the final mathematical equation for the two models turn out to be similar. 


\subsubsection{Boundary layer gas diffusion controlled rate for GM (i.e. diffusion rate of A through gas film)}

The equation for the grain model for boundary layer gas-diffusion is the same as for the shrinking core model.

The boundary layer gas diffusion can be expressed by the following reaction (Levenspiel, 1962):

$\frac{1}{4 \pi R^{2}} \frac{d n_{B}}{d t}=b k_{g}\left(C_{A}-\frac{C_{C}}{K_{E}}\right)$

Rate of consumption of solid ' $\mathrm{B}$ ' $\left(\mathrm{kmol} /\left(\mathrm{m}^{3} \mathrm{~s}\right)\right)$ is given as:

$\dot{N}_{B}=3 b k_{g}\left(C_{A}-\frac{C_{C}}{K_{E}}\right) n \frac{4}{3} \pi R^{2}$

Assuming the volume porosity of the particle to be $\gamma_{p}$ yields

$\dot{N}_{B}=\frac{3 b k_{g}}{\left(1-\gamma_{p}\right) R}\left(C_{A}-\frac{C_{C}}{K_{E}}\right) n\left(1-\gamma_{p}\right) \frac{4}{3} \pi R^{3}=\frac{3 b k_{g}}{\left(1-\gamma_{p}\right) R}\left(C_{A}-\frac{C_{C}}{K_{E}}\right) \varepsilon_{s}$

where $\gamma_{p}=\frac{\text { Volume of pores }}{\text { Total volume of particle }}$

\subsubsection{Diffusion controlled rate due to the pores for grain model.}

The equation for diffusion control through pores for the grain model is similar to diffusion control through the ash layer for the shrinking core model. The equation can be expressed as (Levenspiel, 1962):

$\frac{1}{4 \pi r^{2}} \frac{d n_{B}}{d t}=b D_{e} \frac{d C_{A}}{d r}=$ constant

Here $D_{e}$ is the diffusion coefficient with units of $\mathrm{m}^{2} / \mathrm{s}$.

Integrating with respect to radius ' $r$ ' of the particle again yields: 
$\frac{1}{4 \pi R^{2}} \frac{d n_{B}}{d t}=\frac{b D_{e}}{R^{2}\left(\frac{1}{r_{c}}-\frac{1}{R}\right)}\left(C_{A}-\frac{C_{C}}{K_{E}}\right)$

The rate of consumption of solid ' $\mathrm{B}$ ' $\left(\mathrm{kmol} /\left(\mathrm{m}^{3} \mathrm{~s}\right)\right)$ can be expressed as:

$\dot{N}_{B}=\frac{3 b D_{e}}{\left(1-\gamma_{p}\right) R^{2}\left(\left(\frac{1}{1-X}\right)^{1 / 3}-1\right)}\left(C_{A}-\frac{C_{C}}{K_{E}}\right) \varepsilon_{s}$

\subsubsection{Chemical kinetic controlled rate (for both SC and GM)}

Consider the reaction given by:

$v_{A} A(g)+v_{B} B(s) \rightarrow v_{C} C(g)+v_{D} D(s)$

Chemical reaction rate at the interface is given by:

$$
\frac{t}{\tau}=1-(1-X)^{1 / 3}
$$

Differentiating yields:

$d X / d t=k(1-X)^{2 / 3}$

where $X=\frac{m-m_{D}}{m_{B}-m_{D}}$.

Here $m_{B}$ is the mass when the particle is non reacted (and is present in form of solid $B$ ) and $m_{D}$ is the mass when particle is fully reduced. Also $R_{o}=\left(m_{B}-m_{D}\right) / m_{B}$.

Differentiating Equation 6.35

$\frac{d X}{d t}=\frac{1}{m_{B}-m_{D}} \frac{d m}{d t}=\frac{1}{R_{o} m_{B}} \frac{d m}{d t}=\frac{M W_{B}}{R_{o} m_{B}} \frac{v_{A}}{v_{B}} \frac{d N_{A}}{d t}$

Therefore the rate of consumption of gas ' $A$ ' $\left(\mathrm{kmol} /\left(\mathrm{m}^{3} \mathrm{~s}\right)\right)$ is given as: 
$\dot{n}_{A}=k \frac{v_{B}}{v_{A}} \frac{R_{o} m_{B}}{M W_{B}}(1-X)^{2 / 3}$

The above equation applies to one particle. Considering ' $n$ ' particles in a computational cell yields:

$$
\dot{N}_{A}=n k \frac{v_{B}}{v_{A}} \frac{R_{o} m_{B}}{M W_{B}}(1-X)^{2 / 3}
$$

Also,

$$
n m_{B}=\rho_{B} \varepsilon_{S}\left(Y_{B}+Y_{D} \frac{M W_{B}}{M W_{D}}\right)
$$

Thus, the rate of consumption of gas ' $A$ ' $\left(\mathrm{kmol} /\left(\mathrm{m}^{3} \mathrm{~s}\right)\right)$ is given as:

$$
\dot{N}_{A}=k \frac{v_{B}}{v_{A}} \frac{R_{o}}{M W_{B}} \rho_{B} \varepsilon_{s}\left(Y_{B}+Y_{D} \frac{M W_{B}}{M W_{D}}\right)(1-X)^{2 / 3}
$$




\section{Chapter 7: Simulations of Batch Reactor for Gaseous Fuels}

\section{Introduction}

In this chapter a CFD analysis of gaseous combustion in a fuel reactor of a CL combustor has been presented. The experimental data have been provided by two sources for this study (Son and Kim, 2006, and Mattisson et al., 2001). The Son and Kim (2006) experiments provide the steady state reactor performance at various operating conditions like fluidizing velocity, operating temperature, metal oxide concentrations etc. On the other hand the Mattison et al. (2001) experiments examine the time-varying outlet concentration of flue gases at one fixed operating condition. Both the studies used methane as the fuel with similar reactor dimensions (diameter is between $30 \mathrm{~mm}$ to $50 \mathrm{~mm}$ ) as well as similar fluidizing velocity. Before a full scale CLC system is analyzed, it is important to make sure that a simpler batch reactor can be simulated accurately. In a batch reactor the oxygen carrier particles are exposed to the alternating fuel and oxygen streams. A continuum model was used to describe both the gas and solid phase. Detailed sub-models to account for fluid-particle and particle-particle interaction forces were included. Global models of fuel and carrier chemistry were utilized. The results obtained from CFD were then compared with detailed experimental results. Both the steady and transient CFD simulations provided a reasonable match with the reported experimental data.

In the present simulations the reactors were assumed to operate isothermally and the energy equation for the two phases was not solved. The reasons for this are as follows:

1) The metal oxide reduction reaction inside the fuel reactor is only slightly endothermic which results in only a small drop in temperature (Lyngfelt et al., 2001; Balaji et al., 2010).

2) In the experiments, the reactors were placed in an oven for preheating the inlet gas and to maintain the reactor at the desired experimental temperature (Mattisson et al., 2001; Son and Kim, 2006). The thermal heat flux or temperature boundary conditions that would be needed for the more complete CFD simulations are not clearly available.

\subsection{Details of Son and Kim (2006) Experimental Study}

Son and Kim (2006) used methane as the fuel and fluidizing gas; the metal oxide was a mixture of $\mathrm{NiO}$ and $\mathrm{Fe}_{2} \mathrm{O}_{3}$ supported on bentonite. The mass ratio of metal oxide to bentonite was $60: 40$, and the $\mathrm{NiO}: \mathrm{Fe}_{2} \mathrm{O}_{3}$ mass ratio of the metal oxide mixture varied from $100 \% \mathrm{NiO}$ to $100 \% \mathrm{Fe}_{2} \mathrm{O}_{3}$. The reaction 
rates were determined using thermo gravimetric analysis (TGA). The experiments utilized a closed CLC loop with air and fuel reactors (See Figure 7.1).

The superficial fluidizing velocity in the fuel reactor was varied from $25 \mathrm{~mm} / \mathrm{s}$ to $100 \mathrm{~mm} / \mathrm{s}$ to analyze the effect of $\mathrm{U} / \mathrm{U}_{\mathrm{mf}}$ on the outlet $\mathrm{CH}_{4}$ concentration. The fluidizing gas was pure $\mathrm{CH}_{4}$. Table 7.1 provides the dimensions of the bed, fluidizing velocity and other details of the system used in the simulation. Note that the geometry was axisymmetric, where the bed width is the difference between the outer and inner radii of the FR; see Figure 7.1.

Table 7.1: System properties for experiments of Son and Kim (2006).

\begin{tabular}{|l|l|}
\hline Width of bed (mm) & 16 \\
\hline Height of computational domain $(\mathrm{mm})$ & 900 \\
\hline Range of inlet velocity $(\mathrm{mm} / \mathrm{s})$ & 25 to 100 \\
\hline Mass of metal oxide particle bed $(\mathrm{kg})$ & 0.6 \\
\hline Average diameter of particles (microns) & 128 \\
\hline Average density of particles $\left(\mathrm{kg} / \mathrm{m}^{3}\right)$ & 2000 \\
\hline Initial bed height $(\mathrm{mm})$ & 260 \\
\hline Initial particle volume fraction & 0.6 \\
\hline
\end{tabular}

\subsection{Details of Mattisson et al. (2001) experiments}

The experiments of Mattisson et al. (2001) were carried out in a fixed bed consisting of pure $\mathrm{Fe}_{2} \mathrm{O}_{3}$ granules. The reducing gas was $100 \% \mathrm{CH}_{4}$. The experiment provides the transient variation of outlet concentration of flue gas and hence can be used to check if the dynamic variation of outlet concentration can be predicted by CFD. Table 7.2 provides the dimensions of the bed, fluidizing velocity

Table 7.2: System properties for experiments of Mattisson et al. (2001).

\begin{tabular}{|l|l|}
\hline Width of bed $(\mathrm{mm})$ & 30 \\
\hline Height of computational domain $(\mathrm{m})$ & 0.5 \\
\hline Flow rate $(\mathrm{ml} / \mathrm{min})$ & 300 \\
\hline Mass of metal oxide particles $(\mathrm{g})$ & 90 \\
\hline Average diameter of particles $(\mu \mathrm{m})$ & 215 \\
\hline Average density of particles $\left(\mathrm{kg} / \mathrm{m}^{3}\right)$ & 5700 \\
\hline
\end{tabular}




\subsection{Details of reaction scheme and reaction rates}

Experimental studies by Ryu et al. (2001), Mattisson et al. (2005) and Son and Kim (2006) suggest that during heterogeneous metal oxide reactions the diffusion through the outer boundary layer of the particle and diffusion through the pores is comparatively faster than the complex chemical reactions on the surfaces of the grains.

Mattisson et al. (2001) subject the metal oxide to long cycles of oxidation and reduction. Due to the long reduction extent of the metal oxide, Mattisson et al. report the formation of $\mathrm{Fe}_{3} \mathrm{O}_{4}$ (magnetite) and small quantites of $\mathrm{FeO}$ (wustite) during the reduction cycle. Shi et al. (2008) suggest that the conversion of hematite to wustite takes place in a sequential manner from $\mathrm{Fe}_{2} \mathrm{O}_{3}$ (hematite) to $\mathrm{Fe}_{3} \mathrm{O}_{4}$ (magnetite) and then to $\mathrm{FeO}$ (wustite). The reduction of iron oxide is assumed to occur in two stages as shown below:

$12 \mathrm{Fe}_{2} \mathrm{O}_{3}+\mathrm{CH}_{4} \rightarrow 8 \mathrm{Fe}_{3} \mathrm{O}_{4}+2 \mathrm{H}_{2} \mathrm{O}+\mathrm{CO}_{2}$

$4 \mathrm{Fe}_{3} \mathrm{O}_{4}+\mathrm{CH}_{4} \rightarrow 12 \mathrm{FeO}+2 \mathrm{H}_{2} \mathrm{O}+\mathrm{CO}_{2}$

To model the reduction of nickel oxide, simple one-stage chemical reaction is assumed as follows:

$$
4 \mathrm{NiO}+\mathrm{CH}_{4} \rightarrow 4 \mathrm{Ni}+2 \mathrm{H}_{2} \mathrm{O}+\mathrm{CO}_{2}
$$

Details about the choice of models for simulation of both Son and Kim (2008) and Mattisson et al. (2001) experiments are provided below.

\subsubsection{Reduction rates for first stage (i.e. reduction from $\mathrm{Fe}_{2} \underline{\mathrm{O}}_{3} \underline{\mathrm{to}}_{3} \underline{\mathrm{Fe}}_{4}$ and $\mathrm{NiO}$ to $\mathrm{Ni}$ )}

The reduction rate of $\mathrm{Fe}_{2} \mathrm{O}_{3}$ (hematite) to $\mathrm{Fe}_{3} \mathrm{O}_{4}$ (magnetite) and $\mathrm{NiO}$ to $\mathrm{Ni}$ are determined from thermogravimetric analyzer (TGA) experiments of Son and Kim (2006). Son and Kim suggest that the uniform reaction model can best represent the heterogeneous chemical reactions. The uniform reaction model is given as

$d X / d t=k X^{\prime}$

where $\mathrm{X}$ is the conversion and is given as 


$$
X=\frac{m-m_{r e d}}{m_{o x}-m_{r e d}}
$$

where ' $m_{o x}$ ' is the oxidized (ox) form of an individual particle (i.e. the mass of particle when it is in fully oxidized form given by $\mathrm{Fe}_{2} \mathrm{O}_{3}$ and $\mathrm{NiO}$ ). The reduced form ' $\mathrm{m}_{\text {red }}$ ' (red) of an individual particle is the mass of particle when it is in fully reduced state of Fe and $\mathrm{Ni}$ (Son and Kim, 2006).

Also, $X^{\prime}=\frac{m-m_{r e d, 2}}{m_{o x}-m_{r e d, 2}}$,

where $m_{r e d, 2}$ is the mass of metal oxide particle when it is fully at an intermediate state of reduction (i.e. $\mathrm{Fe}_{3} \mathrm{O}_{4}$ or $\left.\mathrm{Ni}\right)$.

The rate constant in Equation 7.4 is given as $k=a_{r e d} e^{\left(-E_{\text {red }} / R T\right)}$

Son and Kim provide the following values for the pre-exponential factor and the activation energy:

$$
\begin{aligned}
& a_{\text {red }}=0.205-3.93 \times 10^{-3} R_{\mathrm{Fe}_{2} \mathrm{O}_{3}}+1.96 \times 10^{-5} R_{\mathrm{Fe}_{2} \mathrm{O}_{3}}^{2} 1 / \mathrm{s} \\
& E_{\text {red }}=57.1-5.62 \times 10^{-1} R_{\mathrm{Fe}_{2} \mathrm{O}_{3}}+2.79 \times 10^{-3} R_{\mathrm{Fe}_{2} \mathrm{O}_{3}}^{2} \quad \mathrm{KJ} / \mathrm{mol}
\end{aligned}
$$

where $R_{\mathrm{Fe}_{2} \mathrm{O}_{3}}$ is the percentage of iron oxide in the particle (say 25\% iron oxide and $75 \%$ nickel oxide).

Differentiating Equation 7.5 we have:

$$
\frac{d X}{d t}=\frac{1}{m_{o x}-m_{r e d}} \frac{d m}{d t}=\frac{1}{R_{o} m_{o x}} \frac{d m}{d t}
$$

where $R_{o}=\frac{m_{o x}-m_{r e d}}{m_{o x}}$

The equation for $R_{o}$ is modeled as follows

$$
R_{o}=\frac{\left(M W_{\mathrm{Fe}_{2} \mathrm{O}_{3}} \times R_{\mathrm{Fe}_{2} \mathrm{O}_{3}}+M W_{\mathrm{NiO}_{\mathrm{O}}} \times\left(1-R_{\mathrm{Fe}_{2} \mathrm{O}_{3}}\right)\right)-\left(2 M W_{\mathrm{Fe}} \times R_{\mathrm{Fe}_{2} \mathrm{O}_{3}}+M W_{\mathrm{Ni}} \times\left(1-R_{\mathrm{Fe}_{2} \mathrm{O}_{3}}\right)\right)}{\left(M W_{\mathrm{Fe}_{2} \mathrm{O}_{3}} \times R_{\mathrm{Fe}_{2} \mathrm{O}_{3}}+M W_{\mathrm{NiO}} \times\left(1-R_{\mathrm{Fe}_{2} \mathrm{O}_{3}}\right)\right)}
$$


All the loss in mass of metal oxide particle is because of loss of oxygen. Therefore

$d m=d m_{O_{2}}=M W_{O_{2}} \times d n_{O_{2}}$

Also, for every mole of $\mathrm{CH}_{4}$ consumed 2 moles of oxygen is consumed.

$d n_{\mathrm{O}_{2}}=2 d n_{\mathrm{CH}_{4}}$

Using Equations 7.10, 7.11, 7.13 and 7.14 and assuming that there are ' $n$ ' particles per unit volume (' $n$ ' is also called the number density), so that

$\frac{d N_{C H_{4}}}{d t}=n \times k X^{\prime} \frac{R_{o} m_{o x}}{2 M W_{O_{2}}}$

$\frac{d N_{C H_{4}}}{d t}=k X^{\prime} \frac{R_{o} M_{o x}}{2 M W_{O_{2}}}$

where $M_{o x}=n \times m_{o x}$ and $N_{C H 4}=n \times n_{C_{4}}$. Also $M_{o x}$ is the total fully oxidized mass per unit volume and $\frac{d N_{C H_{4}}}{d t}$ is also the amount of methane consumed per unit volume per unit time. Also, the oxidized mass per unit volume can be written as

$M_{o x}=n \times m_{o x}=\rho_{s} \alpha_{s}\left(Y_{\mathrm{Fe}_{2} \mathrm{O}_{3}}+Y_{\mathrm{Fe}_{3} \mathrm{O}_{4}} \frac{v_{\mathrm{Fe}_{2} \mathrm{O}_{3}} M W_{\mathrm{Fe}_{2} \mathrm{O}_{3}}}{v_{\mathrm{Fe}_{3} \mathrm{O}_{4}} M W_{\mathrm{Fe}_{3} \mathrm{O}_{4}}}+Y_{\mathrm{FeO}} \frac{v_{\mathrm{Fe}_{2} \mathrm{O}_{3}} \mathrm{M} W_{\mathrm{Fe}_{2} \mathrm{O}_{3}}}{v_{\mathrm{FeO}} M W_{\mathrm{FeO}}}+Y_{\mathrm{NiO}}+Y_{\mathrm{Ni}} \frac{v_{\mathrm{NiO}} M W_{\mathrm{NiO}}}{v_{\mathrm{Ni}} M W_{\mathrm{Ni}}}\right)$

$M_{r e d, 2}=n \times m_{r e d, 2}=\rho_{s} \alpha_{s}\left(Y_{\mathrm{Fe}_{2} \mathrm{O}_{3}} \frac{v_{\mathrm{Fe}_{3} \mathrm{O}_{4}} M W_{\mathrm{Fe}_{3} \mathrm{O}_{4}}}{v_{\mathrm{Fe}_{2} \mathrm{O}_{3}} M W_{\mathrm{Fe}_{2} \mathrm{O}_{3}}}+Y_{\mathrm{Fe}_{3} \mathrm{O}_{4}}+Y_{\mathrm{FeO}} \frac{v_{\mathrm{Fe}_{3} \mathrm{O}_{4}} M W_{\mathrm{Fe}_{3} \mathrm{O}_{4}}}{v_{\mathrm{FeO}} M W_{\mathrm{FeO}}}+Y_{\mathrm{NiO}} \frac{v_{\mathrm{Ni}} M W_{\mathrm{Ni}}}{v_{\mathrm{NiO}} M W_{\mathrm{NiO}}}+Y_{\mathrm{Ni}}\right)$

$M_{r e d}=n \times m_{r e d}=\rho_{s} \alpha_{s}\left(Y_{\mathrm{Fe}_{2} \mathrm{O}_{3}} \frac{v_{\mathrm{FeO} \mathrm{M}} M W_{\mathrm{FeO}}}{v_{\mathrm{Fe}_{2} \mathrm{O}_{3}} M W_{\mathrm{Fe}_{2} \mathrm{O}_{3}}}+Y_{\mathrm{Fe}_{3} \mathrm{O}_{4}} \frac{v_{\mathrm{FeO}} M W_{\mathrm{FeO}}}{v_{\mathrm{Fe}_{3} \mathrm{O}_{4}} M W_{\mathrm{Fe}_{3} \mathrm{O}_{4}}}+Y_{\mathrm{FeO}}+Y_{\mathrm{NiO}} \frac{v_{\mathrm{Ni}} M W_{\mathrm{Ni}}}{v_{\mathrm{NiO}} M W_{\mathrm{NiO}}}+Y_{\mathrm{Ni}}\right)$ 
$M^{*}=n \times m=\rho_{s} \alpha_{s}\left(Y_{\mathrm{Fe}_{2} \mathrm{O}_{3}}+Y_{\mathrm{Fe}_{3} \mathrm{O}_{4}}+Y_{\mathrm{FeO}}+Y_{\mathrm{NiO}}+Y_{\mathrm{Ni}_{i}}\right)$

where $v_{\mathrm{Fe}_{2} \mathrm{O}_{3}}$ is the stoichiometric coefficient for $\mathrm{Fe}_{2} \mathrm{O}_{3}$ in Equation 19 (i.e $v_{\mathrm{Fe}_{2} \mathrm{O}_{3}}=12, v_{\mathrm{Fe}_{3} \mathrm{O}_{4}}=8$ and $\left.v_{\mathrm{FeO}}=24\right)$. Similarly, from Equation 7.3, $v_{\mathrm{NiO}}$ and $v_{\mathrm{Ni}}$ are both 4.

The rate of consumption of methane $\left(\mathrm{kg} /\left(\mathrm{m}^{3} \mathrm{~s}\right)\right)$ is finally

$\dot{m}_{\mathrm{CH}_{4}}=\frac{k R_{o}}{2 M W_{\mathrm{O}_{2}}} X^{\prime} M_{o x} \frac{X_{\mathrm{CH}_{4}}}{X_{\mathrm{CH}_{4}-T G A}} M W_{\mathrm{CH}_{4}}$

$X_{C_{4} H_{4} T G A}$ is the methane mole fraction in thermo gravimetric analyzer (TGA) experiments used for determining the reaction rates. These rates are scaled linearly for other concentration values. The concentration of $\mathrm{CH}_{4}$ in TGA was $10 \%\left(10 \% \mathrm{CH}_{4}, 10 \% \mathrm{H}_{2} \mathrm{O}, 5 \% \mathrm{CO}_{2}\right.$ and $\left.75 \% \mathrm{~N}_{2}\right)$ or $X_{\mathrm{CH}_{4} \_T G A}=0.1$.

The rate of consumption by $\mathrm{Fe}_{2} \mathrm{O}_{3}$ and $\mathrm{NiO}$ are given as $\left(\mathrm{kg} /\left(\mathrm{m}^{3} \mathrm{~s}\right)\right)$

$\dot{m}_{\mathrm{Fe}_{2} \mathrm{O}_{3}}=R_{\mathrm{Fe}_{2} \mathrm{O}_{3}} \frac{k R_{o}}{2 M W_{\mathrm{O}_{2}}} X^{\prime} M_{o x} \frac{X_{\mathrm{CH}_{4}}}{X_{\mathrm{CH}_{4-} \mathrm{TGA}}} v_{\mathrm{Fe}_{2} \mathrm{O}_{3}} M W_{\mathrm{Fe}_{2} \mathrm{O}_{3}}$
$\dot{m}_{\mathrm{NiO} \mathrm{O}}=\left(1-R_{\mathrm{Fe}_{2} \mathrm{O}_{3}}\right) \frac{k R_{o}}{2 M W_{\mathrm{O}_{2}}} X^{\prime} M_{o x} \frac{X_{\mathrm{CH}_{4}}}{X_{\mathrm{CH}_{4-} \mathrm{TGA}}} v_{\mathrm{NiO}} M W_{\mathrm{NiO}}$

Also $v_{\mathrm{Fe}_{2} \mathrm{O}_{3}}$ is the stoichiometric coefficient for $\mathrm{Fe}_{2} \mathrm{O}_{3}$ in equation 19 (i.e $v_{\mathrm{Fe}_{2} \mathrm{O}_{3}}=12$ ). Similarly, from equation $21, v_{N i O}$ is 4 .

\subsubsection{Reduction rates for second stage (i.e. reduction from $\mathrm{Fe}_{3} \underline{\mathrm{O}}_{4} \underline{\text { to } \mathrm{FeO} \text { ) }}$}

According to Mattisson et al. (2001) small quantities of FeO formation is possible in CLC reactors. The conversion from $\mathrm{Fe}_{3} \mathrm{O}_{4}$ to $\mathrm{FeO}$ can be modeled based on the reaction rates suggested by Shi et al. (2008):

$\frac{1}{M} \frac{d M}{d t}=k_{M} e^{\frac{-E_{M}}{R T}}$ 
where $M$ is the mass of $\mathrm{Fe}_{3} \mathrm{O}_{4}$ per unit volume, $k_{M}$ is the Arrhenius rate coefficient and $E_{M}$ is the activation energy. Also, Shi et al. (2008) suggest that coefficient of the reaction rate for hematite to magnetite is the same as the coefficient of the reduction rate for magnetite to wustite. Based on this assumption it can be shown that:

$k_{M} e^{\frac{-E_{M}}{R T}}=R_{o} k$

where $\mathrm{k}$ and $R_{o}$ are defined by Equations 7.7 and 7.11 respectively. Also, the loss in mass of magnetite and consumption of $\mathrm{CH}_{4}$ is related by

$d m_{\mathrm{Fe}_{3} \mathrm{O}_{4}}=d M=v_{\mathrm{Fe}_{3} \mathrm{O}_{4}} M W_{\mathrm{Fe}_{3} \mathrm{O}_{4}} \times d n_{\mathrm{CH}_{4}}$

After some algebra it can be shown that the rate of consumption of $\mathrm{CH}_{4}\left(\mathrm{~kg} /\left(\mathrm{m}^{3} . \mathrm{s}\right)\right)$ is

$\dot{m}_{\mathrm{CH}_{4}}=\frac{k R_{o}}{v_{\mathrm{Fe}_{3} \mathrm{O}_{4}}} \frac{\rho_{s} \varepsilon_{s} Y_{\mathrm{Fe}_{3} \mathrm{O}_{4}}}{M W_{\mathrm{Fe}_{3} \mathrm{O}_{4}}} \frac{X_{\mathrm{CH}_{4}}}{X_{\mathrm{CH}_{4}-\mathrm{TGA}}} M W_{\mathrm{CH}_{4}}$

The rate of consumption by $\mathrm{Fe}_{3} \mathrm{O}_{4}$ is given as $\left(\mathrm{kg} /\left(\mathrm{m}^{3} . \mathrm{s}\right)\right)$

$\dot{m}_{\mathrm{Fe}_{3} \mathrm{O}_{4}}=\frac{k R_{o}}{v_{\mathrm{Fe}_{3} \mathrm{O}_{4}}} \frac{\rho_{s} \varepsilon_{s} Y_{\mathrm{Fe}_{3} \mathrm{O}_{4}}}{M W_{\mathrm{Fe}_{3} \mathrm{O}_{4}}} \frac{X_{\mathrm{CH}_{4}}}{X_{\mathrm{CH}_{4-} \mathrm{TGA}}} v_{\mathrm{Fe}_{3} \mathrm{O}_{4}} M W_{\mathrm{Fe}_{3} \mathrm{O}_{4}}$

Also $v_{\mathrm{Fe}_{3} \mathrm{O}_{4}}$ is the stoichiometric coefficient for $\mathrm{Fe}_{3} \mathrm{O}_{4}$ in equation 20 (i.e $v_{\mathrm{Fe}_{3} \mathrm{O}_{4}}=4$ ). Table 7.3 provides the final set of equations used in the simulations. 
Table 7.3: Summary of reaction rates used for simulation.

\begin{tabular}{|c|c|}
\hline $\begin{array}{l}\text { Reduction rates } \\
\text { for first stage (i.e. } \\
\text { reduction from } \\
\mathrm{Fe}_{2} \mathrm{O}_{3} \text { to } \mathrm{Fe}_{3} \mathrm{O}_{4} \\
\text { and } \mathrm{NiO} \text { to } \mathrm{Ni} \text { ) }\end{array}$ & 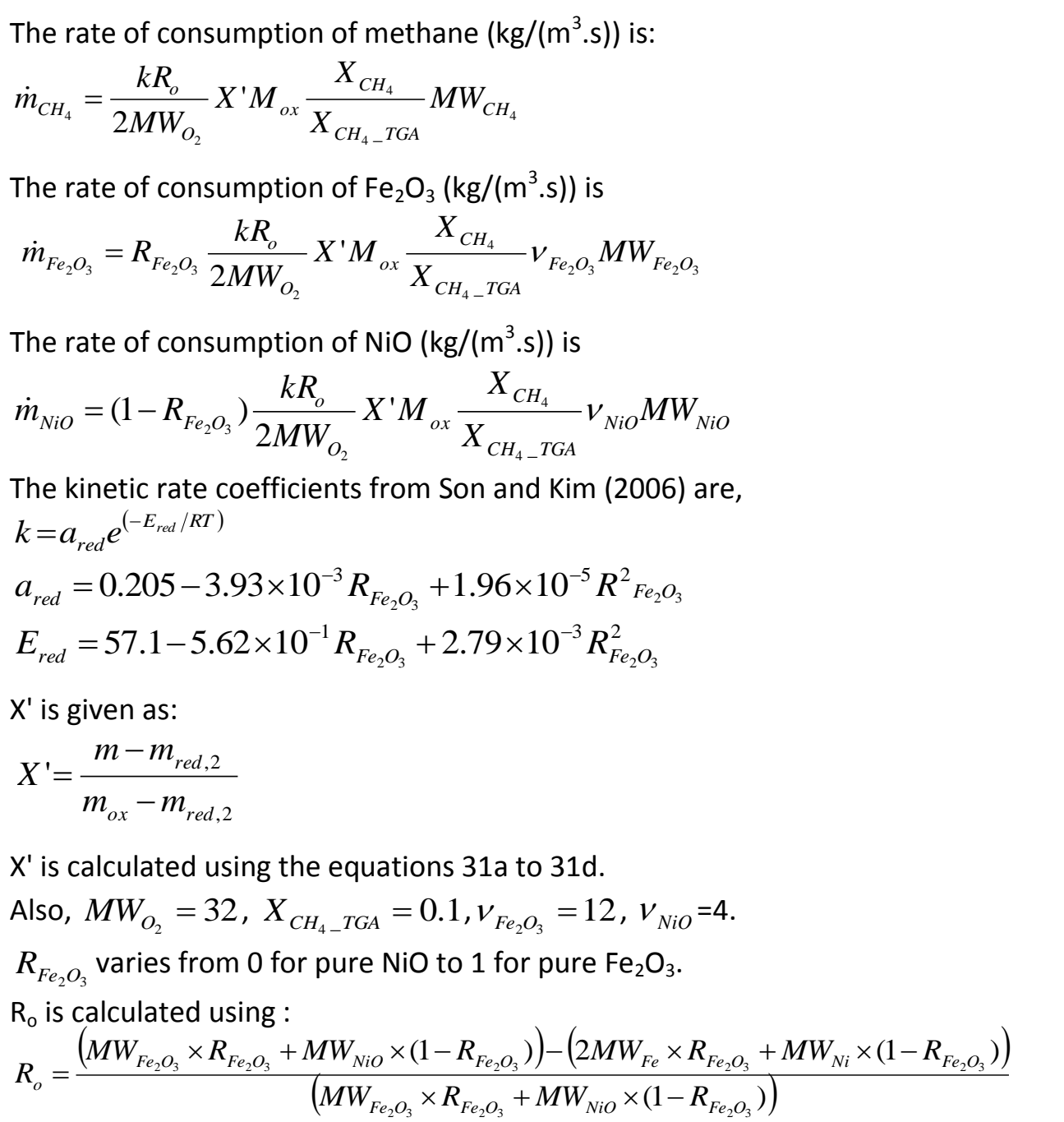 \\
\hline $\begin{array}{l}\text { Reduction rates } \\
\text { for second stage } \\
\text { (i.e. reduction } \\
\text { from } \mathrm{Fe}_{3} \mathrm{O}_{4} \text { to } \\
\mathrm{FeO} \text { ) }\end{array}$ & $\begin{array}{l}\text { The rate of consumption of methane }\left(\mathrm{kg} /\left(\mathrm{m}^{3} . \mathrm{s}\right)\right) \text { is: } \\
\dot{m}_{\mathrm{CH}_{4}}=\frac{k R_{o}}{v_{\mathrm{Fe}_{3} \mathrm{O}_{4}}} \frac{\rho_{s} \varepsilon_{s} Y_{\mathrm{Fe}_{3} \mathrm{O}_{4}}}{M W_{\mathrm{Fe}_{3} \mathrm{O}_{4}}} \frac{X_{\mathrm{CH}_{4}}}{X_{\mathrm{CH}_{4} \mathrm{TGA}}} M W_{\mathrm{CH}_{4}} \\
\text { The rate of consumption by } \mathrm{Fe}_{3} \mathrm{O}_{4}\left(\mathrm{~kg} /\left(\mathrm{m}^{3} . \mathrm{s}\right)\right) \text { is: } \\
\dot{m}_{\mathrm{Fe}_{3} \mathrm{O}_{4}}=\frac{k R_{o}}{v_{\mathrm{Fe}_{3} \mathrm{O}_{4}}} \frac{\rho_{s} \varepsilon_{s} Y_{\mathrm{Fe}_{3} \mathrm{O}_{4}}}{M W_{\mathrm{Fe}_{3} \mathrm{O}_{4}}} \frac{X_{\mathrm{CH}_{4}}}{X_{\mathrm{CH}_{4} \_T G A}} v_{\mathrm{Fe}_{3} \mathrm{O}_{4}} M W_{\mathrm{Fe}_{3} \mathrm{O}_{4}} \\
\text { Also, } v_{\mathrm{Fe}_{3} \mathrm{O}_{4}}=4 \text { and } X_{\mathrm{CH}_{4}-T G A}=0.1 . \\
\text { Based on studies of Shi } \text { et al. (2008), the same reaction rate coefficients for } \\
\text { reduction of hematite to magnetite and for reduction from magnetite to wustite } \\
\text { are assumed. The values for rate coefficient ' } \mathrm{k} \text { ' have been given in the first stage } \\
\text { summary above. }\end{array}$ \\
\hline
\end{tabular}




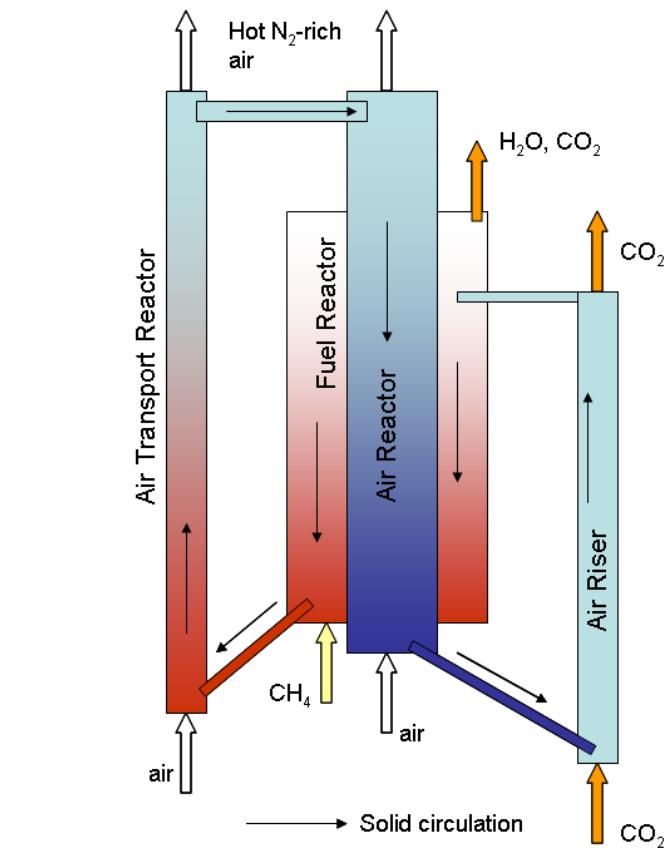

a) Complete CLC system

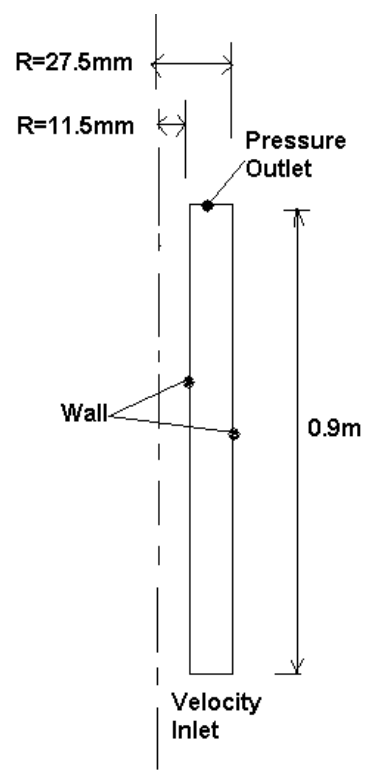

b) Computational domain for CFD of FR.

Fig. 7.1: Full CLC system studied by Son and Kim (2006).

\subsection{Details of Numerics for Son and Kim (2006) experiments and Grid Convergence Studv:}

The grid used in the simulation was a structured rectangular grid. Table 7.4 provides details about the grid size and numerics used in the simulations. The CFD domain for the annular geometry of the FR has been presented in Fig. 7.1. Only the FR has been simulated in the present study. The fuel reactor can be simulated in a decoupled manner from the air reactor because of the following reasons:

1) The rate of consumption of metal oxide ( $1 \mathrm{gram} / \mathrm{s}$ ) due to reaction with methane is much less than the total inventory of metal oxide inside the reactor $(0.5 \mathrm{~kg})$. This results in a long time period (several minutes) for metal oxide to be completely consumed by the metal oxide in the FR.

2) The reactor achieves stable outlet concentration of flue gases $\left(\mathrm{CO}_{2}, \mathrm{CH}_{4}\right.$ etc.) in just a few seconds ( $<5$ seconds) of operation (see Fig. 7.3).

3) The rate of solid circulation is relatively small ( $2.9 \mathrm{gram} / \mathrm{s})$ in comparison with the metal oxide inventory in the reactor $(0.5 \mathrm{~kg})$. Therefore in the present simulations, solid circulation has not been simulated. 
One uncertain parameter in the simulation is the average steady state conversion of the metal oxide in the experimental reactor as it has not been reported by Son and Kim (2006). However it is expected that the conversion extent would be relatively high $\left(X^{\prime}>0.9\right)$, the reasons for which are discussed below:

The mass flow rate of methane in the FR is $1.75 \times 10^{-5} \mathrm{~kg} / \mathrm{s}$ at an inlet flow velocity of $50 \mathrm{~mm} / \mathrm{s}$. Assuming complete oxidation of the fuel, the rate of consumption of $\mathrm{NiO}$ can be found from equation 21 to be 0.32 grams/s. Similarly the maximum rate of consumption of $\mathrm{Fe}_{2} \mathrm{O}_{3}$ can be estimated from equation 19 to be 2.1 grams/s. Thus the rate of consumption of metal oxide is in general much smaller than the steady state mass of the metal oxide inside the bed $(0.5 \mathrm{~kg})$. Also, the reported experimental solid circulation rate of metal oxide in the experiments is in range of 2.9 grams $/ \mathrm{s}$ (or $13 \mathrm{~kg} / \mathrm{m}^{2} / \mathrm{s}$ of mass flux in riser). This indicates that any metal oxide reduced by methane will quickly be replaced by fresh metal oxide from the air reactor leading to a high average steady state metal oxide conversion in the fuel reactor (i.e. $X^{\prime}>0.9$ ). The steady state conversion level maintained in the FR has not been reported by Son and Kim. However other experimental, theoretical and numerical studies of CLC reactors indicate high steady state conversion $\left(X^{\prime}>0.9\right)$ in the fuel reactor (Lyngfelt et al., 2001; Abad et al., 2006; Mahalatkar et al., 2010). In the present simulations it has been assumed that the conversion in the reactor is initially 1 and the simulations have been further run for 15 seconds with time averaging of concentrations between 5 and 15 seconds. Fig. 7.2 shows the time varying concentration of $\mathrm{CH}_{4}$ at the outlet of the fuel reactor for a pure $\mathrm{NiO}$ and pure $\mathrm{Fe}_{2} \mathrm{O}_{3}$ particle; a relatively stable outlet concentration of methane is achieved in just a few seconds of simulation time during which time only a relatively small amount of $\mathrm{NiO}$ or $\mathrm{Fe}_{2} \mathrm{O}_{3}$ will be consumed $\left(\Delta \mathrm{X}^{\prime}<0.1\right)$.

The widths of the reactor as well as the fluidizing velocity are very similar for the experiments of Son and Kim (2006) and Mattisson et al. (2001). The Son and Kim (2006) experiment was first considered for the grid independence as it had the highest fluidizing velocity $(100 \mathrm{~mm} / \mathrm{s})$. The fluidizing velocity for the experiments of Mattisson et al. (2001) was less than $10 \mathrm{~mm} / \mathrm{s}$ and the experiments were fixed bed where there was no need to resolve the dynamics of the solid phase. Therefore a mesh providing grid independence for the Son and Kim (2006) experiments is also likely to provide grid independence for other experiments. Table 7.4 provides a comparison of coarse and fine mesh Son and Kim simulations. 
Fig. 7.3 compares the results from a coarse grid simulation using lower order discretization with a fine grid simulation using higher order discretization. The maximum difference in outlet concentration of $\mathrm{CO}_{2}$ as predicted by coarse and fine grid is within $5 \%$ at a superficial velocity of $50 \mathrm{~mm} / \mathrm{s}$ (Fig. 7.3).

From the above discussion it has been concluded that the grid point density and discretization scheme used for the fine grid will also be sufficient for experiments of Mattisson et al. (2001) where the superficial velocities are much lower.

Table 7.4: Details of coarse and fine grids for simulations of Son and Kim (2006) experiments.

\begin{tabular}{|l|c|c|}
\hline & Coarse Grid & Fine Grid \\
\hline No of Cells & $10000(50 \times 200)$ & $40000(50 \times 800)$ \\
\hline $\begin{array}{l}\text { Spatial discretization scheme for each } \\
\text { Phase and Species }\end{array}$ & QUICK & QUICK \\
\hline $\begin{array}{l}\text { Spatial discretization scheme for } \\
\text { Momentum Equation }\end{array}$ & Second Order Upwind & Second Order Upwind \\
\hline Time integration scheme & Second Order Implicit & Second Order Implicit \\
\hline Time step size & 0.00002 sec & 0.00002 sec \\
\hline Iterations per time step & 20 iterations & 20 iterations \\
\hline
\end{tabular}

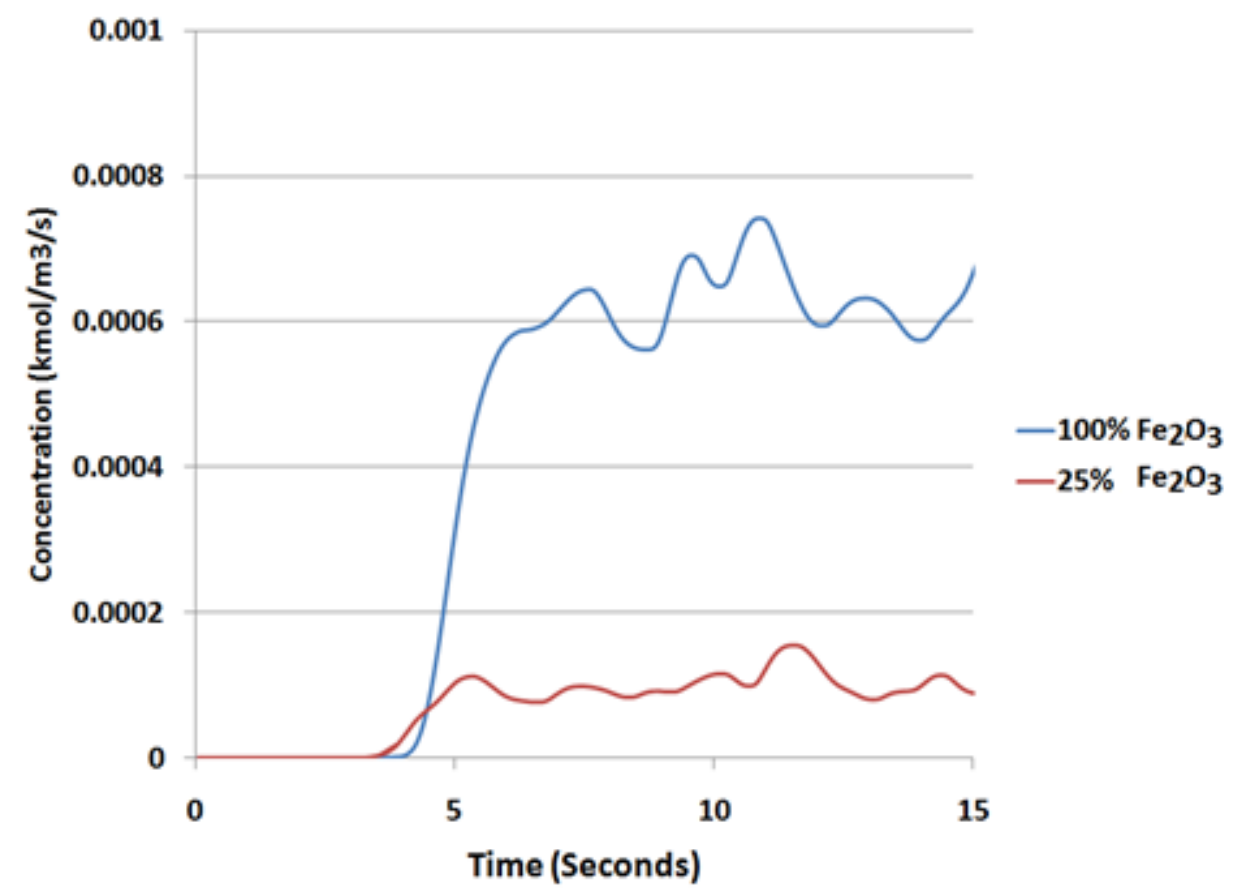

Fig. 7.2: Time variation of $\mathrm{CH} 4$ concentration (superficial velocity of $25 \mathrm{~mm} / \mathrm{sec}$ and operating temperature of 1123K). 


\subsection{Details of Numerics for Mattisson et al. (2001) experiments}

The fuel reactor for the experiments of Mattisson et al. (2001) has a cylindrical geometry. The grid used in the simulation was a rectangular grid. Table 7.5 provides details about the grid size and numerics used in the simulations. To resolve the solid-solid frictional stress in the momentum equation of the solid phase, a low time step would be required. In the experiments of Mattisson et al. (2001) a fixed bed was used so the velocity for the solid phase was fixed to zero. The solid phase momentum equation was not solved. Therefore a larger time step could be used for this experiment in comparison with simulations of the experiment of Son and Kim (2006).

Table 7.5: Numerics for simulation of Mattisson et al. experiments (2001)

\begin{tabular}{|l|l|}
\hline Spatial mesh size spacing & $50 \times 100(\mathrm{H} \times \mathrm{V})$ \\
\hline Horizontal grid expansion factor & 1 (Uniform) \\
\hline Vertical grid expansion factor & 1.02 \\
\hline $\begin{array}{l}\text { Spatial discretization scheme for each } \\
\text { Phase conservation equation }\end{array}$ & QUICK (Fluent) \\
\hline Spatial discretization of other equations & Second Order Upwind \\
\hline Time integration scheme & Second Order Implicit \\
\hline Time step & 0.02 seconds \\
\hline Iterations per time step & 20 \\
\hline
\end{tabular}

\subsection{Results: Son and Kim experiments (2006)}

Fig. 7.4 shows the comparison of the computed outlet flue gas concentration with the experiment for varying values of the $\mathrm{NiO}: \mathrm{Fe}_{2} \mathrm{O}_{3}$ composition in the metal oxides. The outlet concentration of $\mathrm{CO}_{2}$ shows less than $2 \%$ error for high concentrations of $\mathrm{NiO}$ in metal oxide (>50\%). However, at high $\mathrm{Fe}_{2} \mathrm{O}_{3}$ concentration the error in $\mathrm{CO}_{2}$ concentration increases to $10 \%$. Complex chemical reactors such as that developed by Son and Kim (2006) usually report some variation in the outlet concentration between different runs. For example Fryer and Potter (1976) have used a simple bubbling bed reactor to analyze the decomposition of ozone. They report variation in outlet concentration of more than $15 \%$ between different runs at the same operating conditions. Similarly Mattisson et al., $(2001,2003)$ report changes in reaction rate of metal oxide particles with time. The changes in reaction rate are due to physical and chemical changes of the particles such as, attrition, annealing, change in grain size etc. Leion et al. 
(2008) also report considerable change in the outlet concentration of flue gases such as $\mathrm{CO}_{2}, \mathrm{CH}_{4}$ etc over different runs in their CLC reactor utilizing solid fuels. Son and Kim do not provide error estimates for the outlet concentration of gases in their experiments, which to some extent inhibits the determination of cause for the differences between experiments and the present simulation.

The differences in the outlet methane concentrations in Fig. 7.4 are attributed to reasons listed below:

1) The chemistry model used is a curve fit to available experimental data from a thermogravimetric analyzer (TGA). The TGA experiments were carried out at a single concentration of methane by flooding the reaction chamber with excess amount of gases (Son and Kim, 2006). In the actual reactor system, the methane concentration will vary from close to $0 \%$ to $100 \%$. A linear scaling in reaction rates has been assumed with respect to concentration of methane, which may not necessarily be accurate.

2) Error in the simulation, such as error in the bubble size or frequency, can cause the outlet concentration to vary. The bubbles allow the leakage of gases through the bed. However, detailed simulations (Hulme et al. 2005 ; Chandrasekaran et al. 2005) have shown that the fluid mechanics can be reasonably predicted by using mathematical models that are similar to that used in the present study; see Chapter 5.

3) The changes in reaction rate of the metal oxide particle that are caused due to physical and chemical changes of the particle over time such as, attrition, annealing, change in grain size etc.

4) In Fig. 7.4 the error in outlet flue gas concentration is higher in the case of $100 \% \mathrm{Fe}_{2} \mathrm{O}_{3}$ particles and lower for particles with $\mathrm{NiO}$. This is attributed primarily to the uncertainty in the average steady state conversion extent of the metal oxide (i.e. $\mathrm{X}^{\prime}$ ). A greater amount of $\mathrm{Fe}_{2} \mathrm{O}_{3}$ is required to consume the same amount of methane in comparison with $\mathrm{NiO}$. This need for a greater amount of $\mathrm{Fe}_{2} \mathrm{O}_{3}$ is due to the larger stoichiometric coefficient of $\mathrm{Fe}_{2} \mathrm{O}_{3}$ in equation 7.1 as well as its larger molecular weight. Because of the faster rate of consumption of $\mathrm{Fe}_{2} \mathrm{O}_{3}$, the average steady state conversion of the metal oxide particle in the experimental study could be less than the assumed value of $X^{\prime}>0.9$ (See section 3.1 for more details). As discussed in section 7.4, the simulations of the FR were started by assuming the conversion to be 1.0 (i.e. $X^{\prime}=1$ ) and allowing the conversion to drop slightly as the reaction proceeded $\left(\Delta X^{\prime}<0.1\right)$. 
Fig. 7.5a shows a sample contour plot of the gas phase void fraction; regions of high gas concentration correspond to the location of bubbles. Fig. 7.5b shows a corresponding sample contour plot of methane mole fraction. As expected the bubbles have a high concentration of methane and provide a mechanism for leakage of fuel (incomplete combustion). Fig. 7.5c shows the corresponding mole fraction plot of $\mathrm{CO}_{2}$. The $\mathrm{CO}_{2}$ concentration is low at the inlet region but increases with bed height as the methane reacts with the metal oxide. Comparing Fig. $7.5 \mathrm{a}$ and $7.5 \mathrm{c}$ it can be observed that $\mathrm{CO}_{2}$ concentration is higher in the emulsion phase and low in the regions where bubbles are present. This can be expected as the bubble region contains large quantities of methane and low concentrations of metal oxide which is likely to reduce the reaction rates.

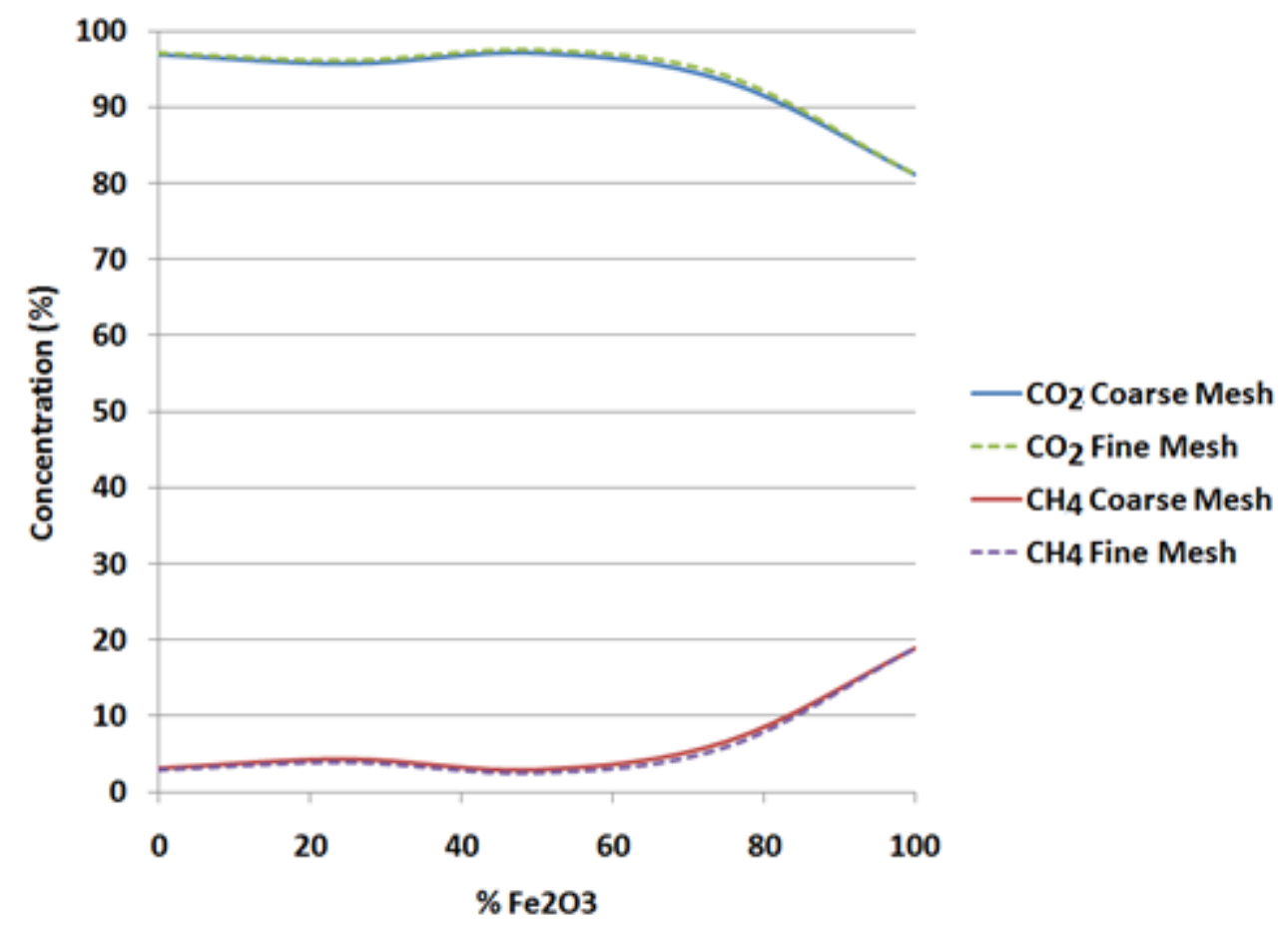

Fig. 7.3: Fine vs. Coarse grid (superficial velocity of $50 \mathrm{~mm} / \mathrm{sec}$ and operating temperature of 1123K). 


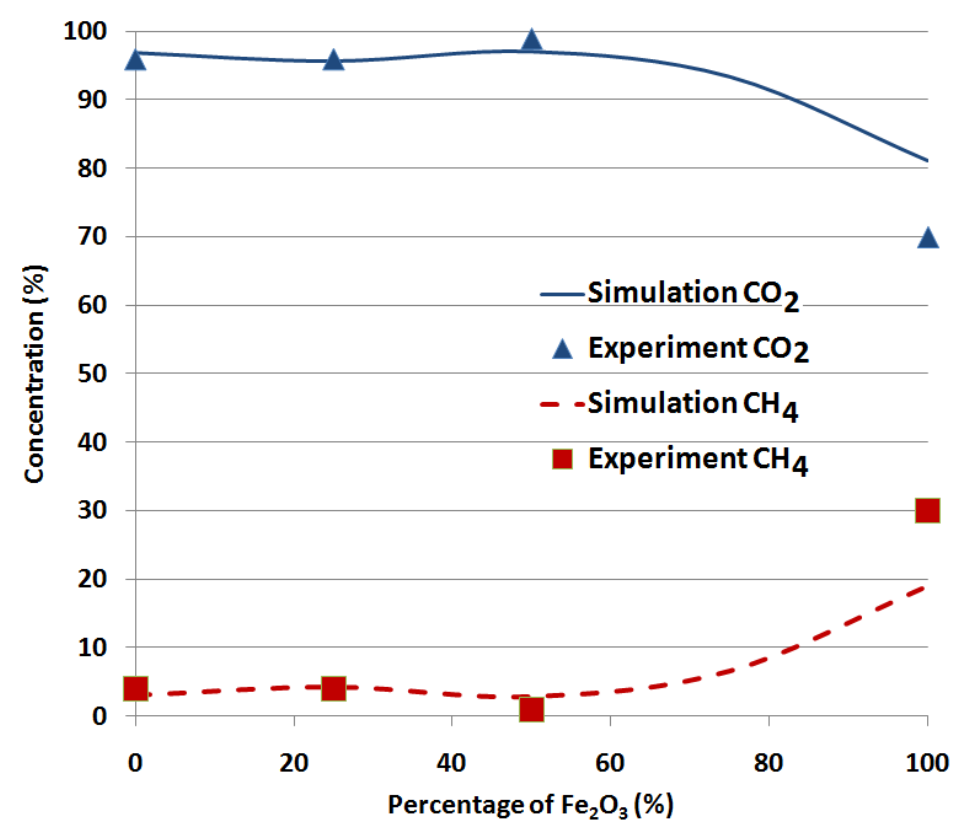

Fig. 7.4: Outlet composition of flue gases at various $\mathrm{Fe}_{2} \mathrm{O}_{3}$ concentration for Son and $\mathrm{Kim}$ experiments (2006), (100\% indicates only $\mathrm{Fe}_{2} \mathrm{O}_{3}$ is present and $0 \%$ indicates only $\mathrm{NiO}$ is present).

Fig. 7.6 shows the phase contour plots at three different velocities. Due to the tall height and relatively small distance between walls of the reactor, the bubbles formed near the distributor plate of the reactor grow into slugs. The formation of the slugs is due to coalescence of smaller bubbles. The behavior of the bubble coalescence is similar to that reported by Kunii and Levenspiel (1991). Near the distributer plate small bubbles are formed due to instabilities in the flow and they tend to coalesce with other adjacent smaller bubbles and grow larger. Coalescence is also observed in the upper portion of the bed. Here the coalescence is mainly between two vertically offset bubbles one of which is trailing the other. If a smaller bubble is trailing this larger bubble then the wake of the bubble allows the trailing smaller bubble to accelerate and merge with the larger bubble. Also in general larger bubbles tend to have a higher velocity than smaller bubbles. Therefore they tend to merge with any smaller bubbles that are in their path as they rise up. Due to the narrow flow passage area the size of the bubbles grows quickly to be roughly the distance between the inner and outer walls of the annular reactor resulting in the formation of slugs. Also, considerably larger bed expansion can be seen in the system at higher velocities (Fig. 7.6). At low superficial velocity of $25 \mathrm{~mm} / \mathrm{s}$ it is observed that the slugs mainly rise between the walls of the reactor and there is no direct contact between the slugs and the walls of the reactor (Fig. 7.6a). At higher velocity of $50 \mathrm{~mm} / \mathrm{s}$ the slugs come in contact with the walls and tend to slide up the wall 
(Fig. 7.6b). At even higher velocity of $100 \mathrm{~mm} / \mathrm{s}$ it is observed that the slugs have grown to span the entire distance between the walls (Fig. 7.6c). This causes the upper portion of the bed to be alternating layers of gas and emulsion regions. These observations are similar to that experimentally reported by Clift and Grace (1985) for slug flow reactors. Also, rise of the slugs causes the solid particles to be pushed upwards. These particles then subsequently move back down into the bed along the walls of the reactor. This pattern seems to cause a core-annulus region similar to that observed in a transport bed reactor. The velocity vectors of solid particles along the walls are primarily downwards, and in the central region between the walls the velocity vectors are primarily upwards.

Fig. 7.7a shows the computed solid volume fraction at one time instant, for the lowest gas velocity. Low solid volume fractions with volume fraction of close to zero are the bubbles and slugs. Fig. 7.7b shows the corresponding gas velocity vectors. It is observed that the gas velocity vectors are relatively large inside the bubbles and smaller in the emulsion. The large gas velocities in the bubbles indicate that they are what is referred to as "fast bubbles" (Kunni and Levenspiel, 1991); in this case the bubbles move significantly faster than the gas in the emulsion phase. Kunni and Levenspiel (1991) also point out that fast moving bubbles form a "cloud" and the gas inside the bubble gets re-circulated inside this cloud region surrounding the bubble. Thus formation of fast bubbles can act as conduit for methane to bypass the bed by circulating in a limited region inside or around the fast moving bubble. Fig. 7.7c shows the rate of consumption of $\mathrm{CH}_{4}$ due to reaction with $\mathrm{NiO}$ in $\mathrm{kmol} / \mathrm{m}^{3} / \mathrm{s}$. It is observed that the reaction rate is higher in the emulsion region where most of the solid particles are present and low in the bubble regions where the solid particles are almost non-existent.

Fig. 7.8 shows the comparison between simulation and experiment for an outlet flue gas concentration at different velocities for a $75 / 25 \%$ ratio of $\mathrm{NiO}$ to $\mathrm{Fe}_{2} \mathrm{O}_{3}$. The maximum error in the $\mathrm{CO}_{2}$ concentration is less than $5 \%$. Also, the observed difference in concentration increases with the increase in velocity. This is not surprising as the bubble frequency is larger at higher velocity and the bubbles will allow by-passing of some methane without combustion. The bubble size predicted by the numerical model depends on many different parameters such as the fluid-particle drag law, rheological models in the kinetic and frictional flow regimes, etc. A larger bubble size will have a higher effect on the outlet flue gas concentration, and the error in bubble size prediction that can be expected of any numerical model will result in a corresponding error in the concentration of methane. 
Fig. 7.9 shows the comparison between simulation and experiment for the outlet flue gas concentrations at different temperature for a $75 / 25 \%$ ratio of $\mathrm{NiO}$ to $\mathrm{Fe}_{2} \mathrm{O}_{3}$. The error in methane concentration is highest at low temperature. This is again attributed to the uncertainty in the average conversion extent of the metal oxides in the experimental reactor as well as unavailability of error estimates for the experimental study. In general it is observed that the experimental concentrations of $\mathrm{CH}_{4}$ at outlet are lower than the predicted values. This may be likely due to higher average steady state conversion extent of the metal oxides $\left(X^{\prime}\right)$ inside the experimental reactor (See section 7.4 for more details regarding $\mathrm{X}^{\prime}$ values assumed in the simulations). The $\mathrm{CO}_{2}$ concentrations predicted by simulations match the experimental results within an error of $10 \%$ at most simulation points. The error is highest at a temperature of $1223 \mathrm{~K}$. This is because in the experiments there was large concentration of cO most likely because of dissociation of $\mathrm{CO}_{2}$. The formation of $\mathrm{CO}$ has been ignored in the present numerical simulations.

\subsection{Results: Mattisson et al. (2001) experiments}

Fig. 7.10 compares the simulated and experimental outlet concentration variation of $\mathrm{CO}_{2}$ and $\mathrm{CH}_{4}$ when the formation of wustite (FeO) is ignored in the simulation. Balaji et. al., (2010) have recently simulated the Mattisson et al. (2001) experiments using a simpler model based on thermodynamic equilibrium that is solved in MATLAB ${ }^{\mathrm{TM}}$. The results from Balaji et al., are also provided in Fig. 7.10 for comparison with the present simulations. At the start of simulation the entire metal oxide is in the form of hematite $\left(\mathrm{Fe}_{2} \mathrm{O}_{3}\right)$ because of which the methane is consumed almost entirely and the outlet $\mathrm{CO}_{2}$ concentrations are high. After about 360 seconds of simulation time almost all of the hematite $\left(\mathrm{Fe}_{2} \mathrm{O}_{3}\right)$ is reduced to magnetite $\left(\mathrm{Fe}_{3} \mathrm{O}_{4}\right)$ (Fig. 7.11). Since further reduction to wustite $(\mathrm{FeO})$ is ignored (in this set of simulations, Fig. 7.10), there is little consumption of methane at the end 360 seconds due to unavailability of $\mathrm{Fe}_{2} \mathrm{O}_{3}$. The experimental and simulated outlet concentration of $\mathrm{CO}_{2}$ and $\mathrm{CH}_{4}$ show the similar trends until about 200 seconds (Fig. 7.10). At this point the concentration of magnetite $\left(\mathrm{Fe}_{3} \mathrm{O}_{4}\right)$ becomes considerable and the reduction of magnetite to wustite becomes important (Fig. 7.11). So these initial simulations do not follow the experimental trends beyond this time, due to the lack of including the reaction for reduction of magnetite $\left(\mathrm{Fe}_{3} \mathrm{O}_{4}\right)$ to wustite $(\mathrm{FeO})$.

Fig.7.12 compares the simulated and experimental outlet concentration variation of $\mathrm{CO}_{2}$ and $\mathrm{CH}_{4}$ when the formation of wustite $(\mathrm{FeO})$ is included in the simulation, again using the rate data from Son and Kim (2006) for the hematite to magnetite reduction, but also assuming that the magnetite to 
wustite reaction has the same rate coefficient (Shi et al., 2008). Some variability of these rate coefficients are expected based on the work of Mattisson et al. $(2001,2003)$ and Leion et al. (2008). For the first 150 seconds of simulation time the outlet concentrations are similar to those observed in Fig. 7.10. After 300 seconds, the reduction of magnetite to wustite starts to become important. The large $\mathrm{CO}_{2}$ concentration at the end of 360 seconds of simulation time is primarily because of reduction of magnetite $\left(\mathrm{Fe}_{3} \mathrm{O}_{4}\right)$ to wustite ( $\mathrm{FeO}$ ) by methane. In general, the results obtained from the present CFD simulations provide a better match with the trends observed in the experimental study in comparison with that of Balaji et al. (Fig. 7.10 and Fig. 7.12). It appears that significantly better agreement with the experimental data could be achieved by tuning the kinetic rate constants; e.g.; by reducing both sets of rate constants.

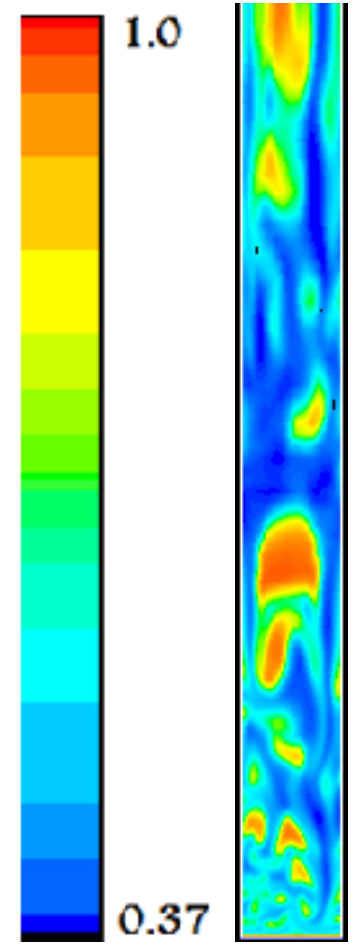

a) Volume Fraction Gas Phase

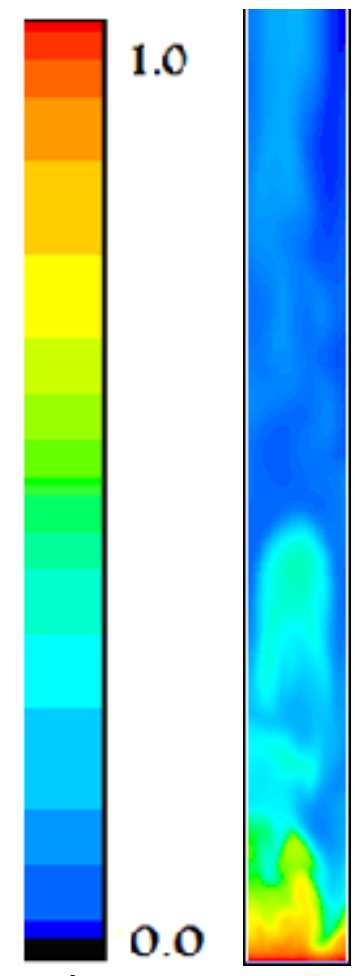

b) Mole Fraction $\mathrm{CH}_{4}$

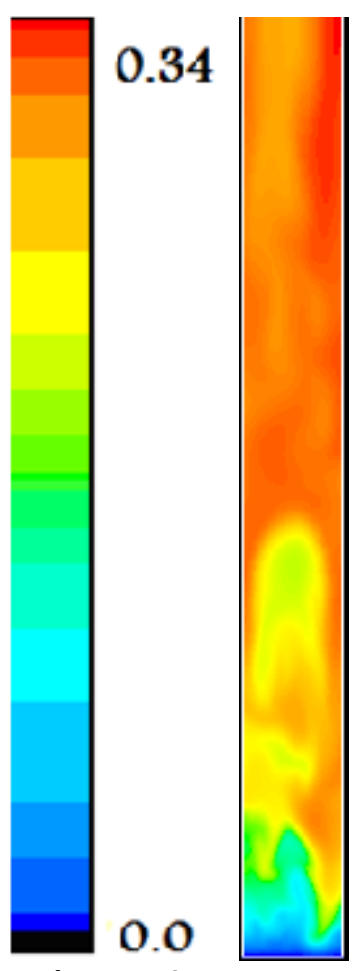

c) Mole fraction $\mathrm{CO}_{2}$

Fig. 7.5: Contour Plots for Son and $\operatorname{Kim}(2006)$ experiments $[\mathrm{NiO}-100 \%$, velocity $=50 \mathrm{~mm} / \mathrm{s}$ ]. 


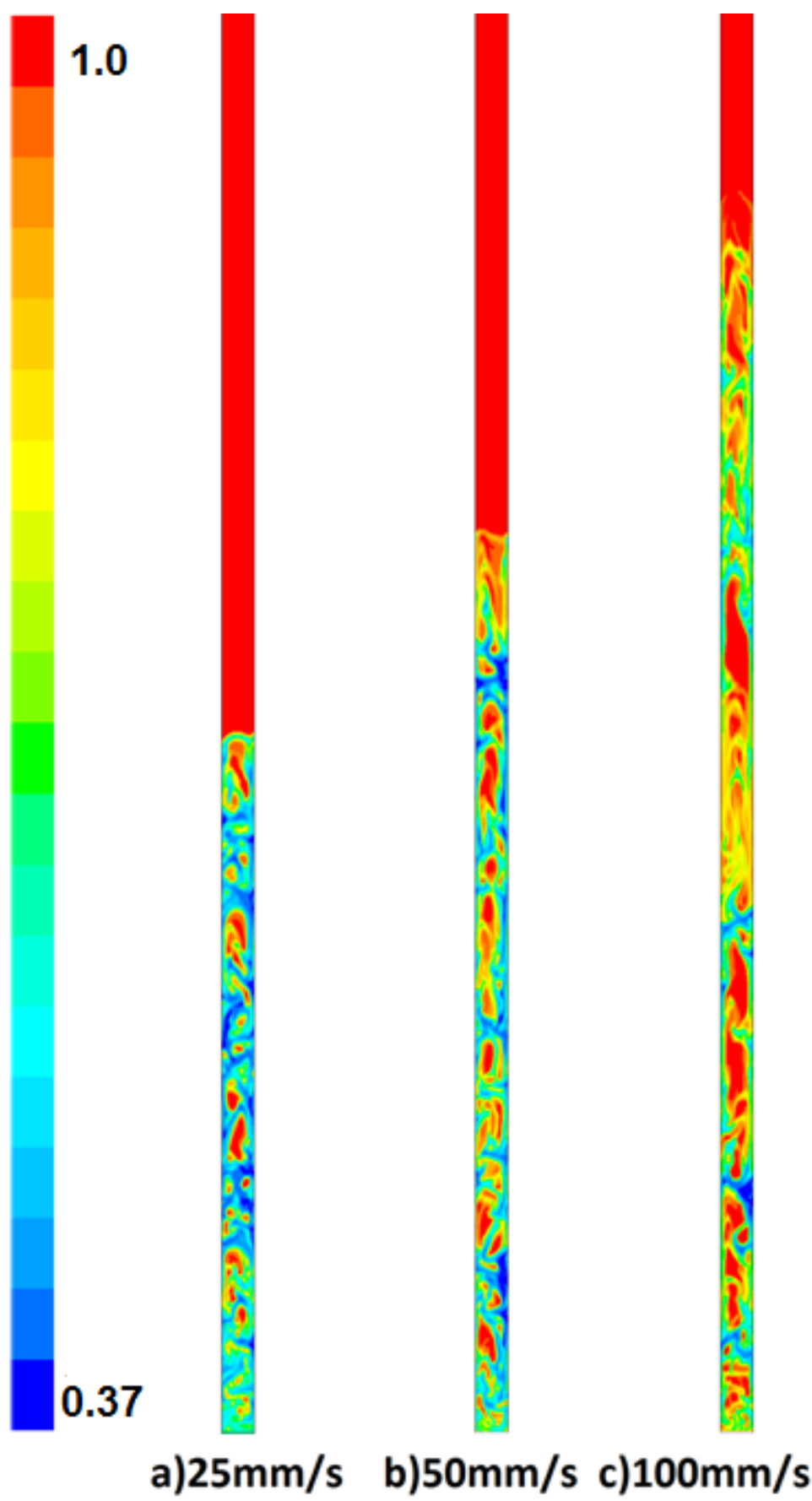

Fig. 7.6: Gas phase volume fraction contour for Son and Kim (2006) experiments for three different gas velocities [NiO-75\%, Temperature $=1123 \mathrm{~K}$ ]. 

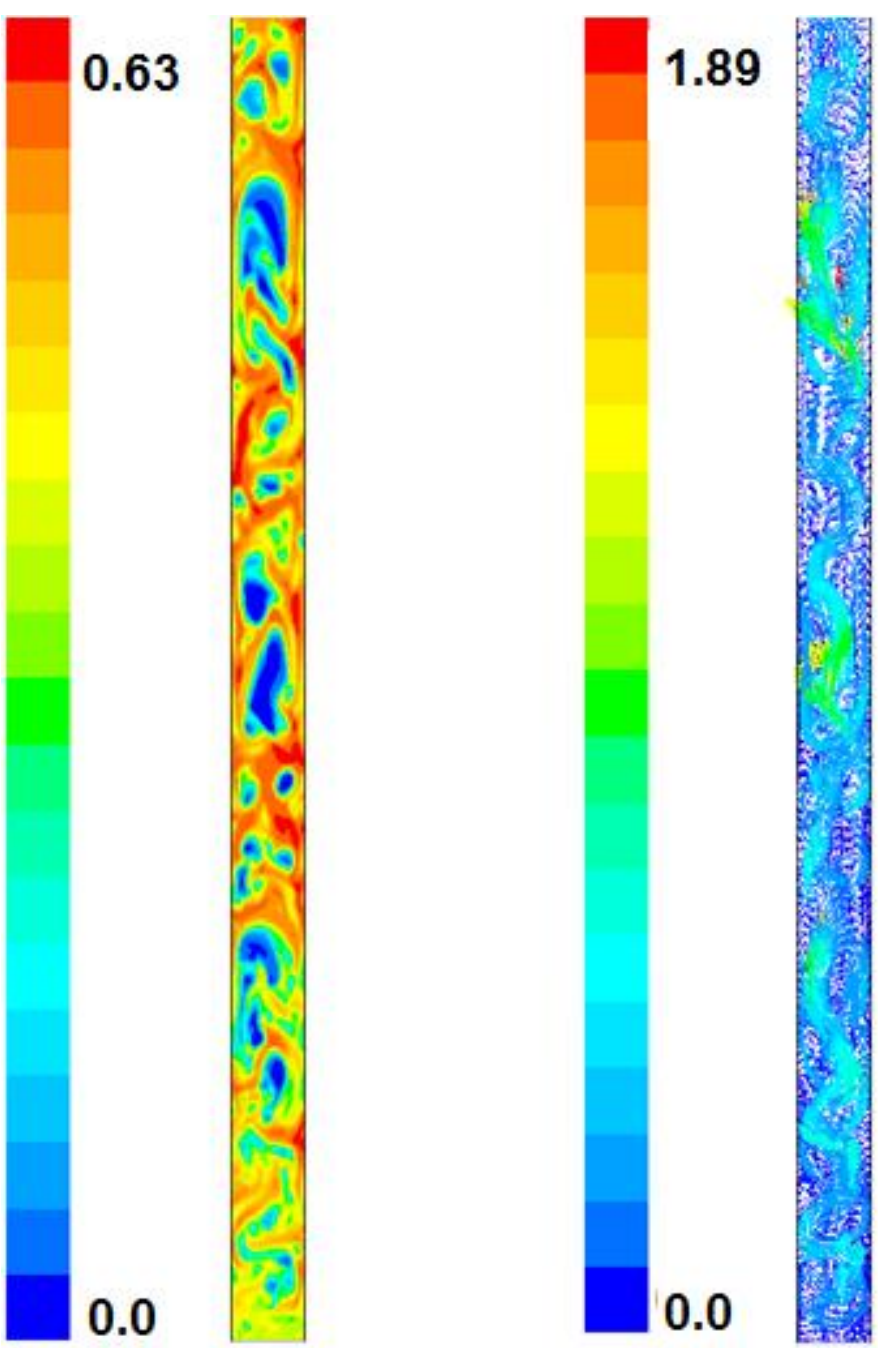

b) Gas velocity vectors

a) Volume Fraction Solid Phase

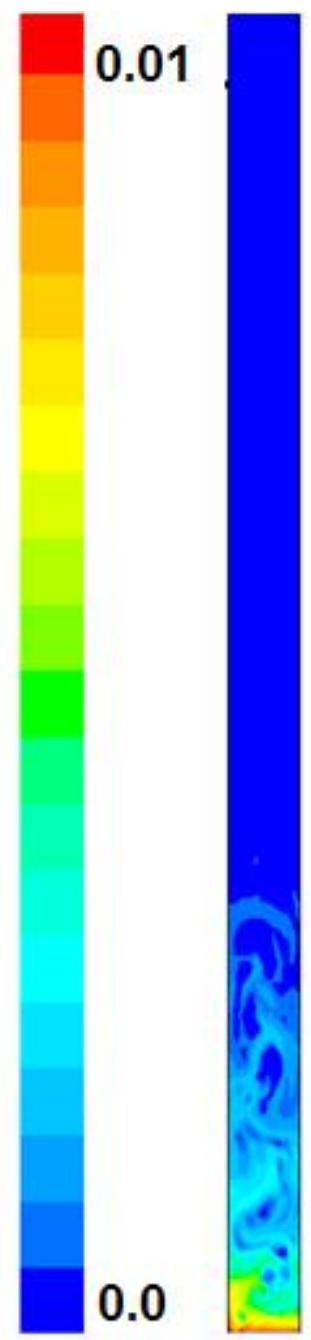

c) $\mathrm{NiO}-\mathrm{CH}_{4}$ Reaction Rate

Fig. 7.7: Contour and Vector Plots for Son and Kim (2006) experiments [NiO-75\%, velocity $=25 \mathrm{~mm} / \mathrm{s}$, Temperature $=1123 \mathrm{~K}]$. Computational domain is truncated to show the bubble regions and gas velocity vectors. 


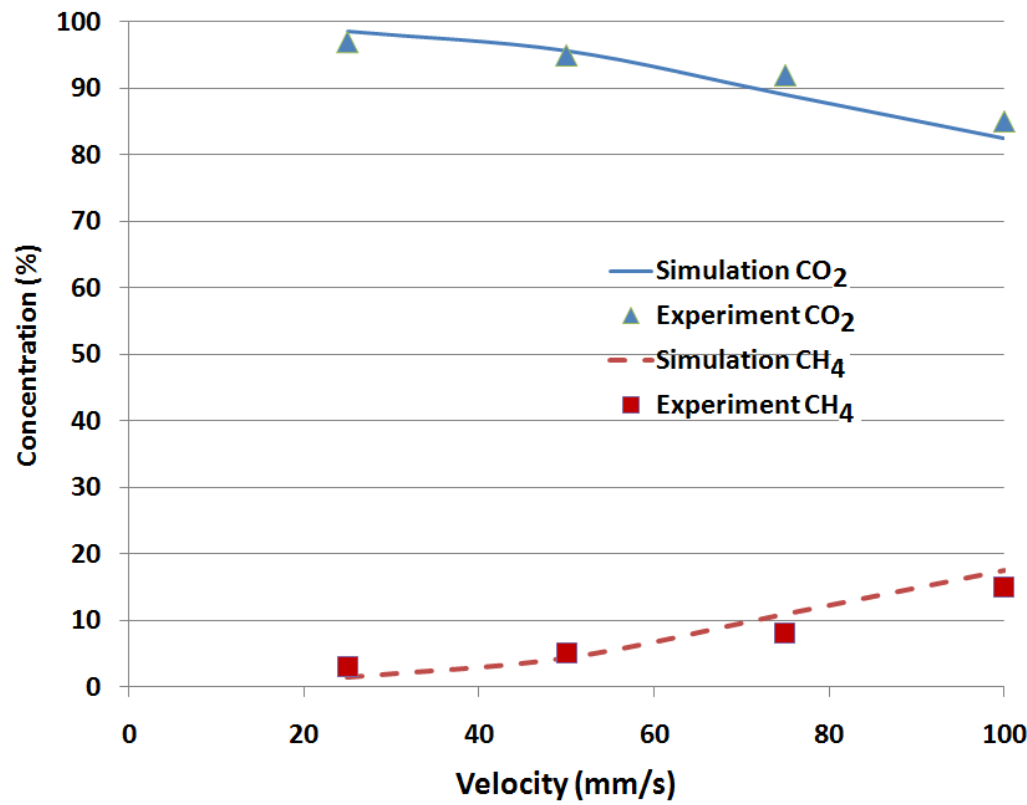

Fig. 7. 8: Outlet composition of flue gases at various velocities for Son and Kim (2006) experiments (NiO: $\mathrm{Fe}_{2} \mathrm{O}_{3}:: 75: 25$ ).

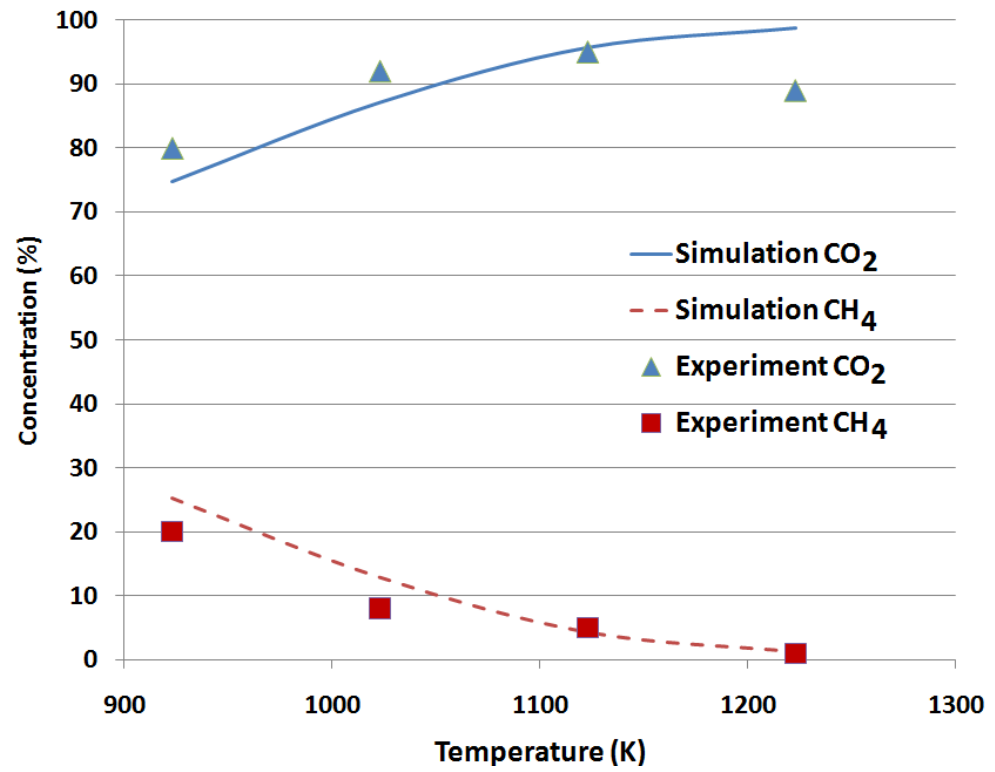

Fig. 7. 9: Variation in Concentration with Temperature. 


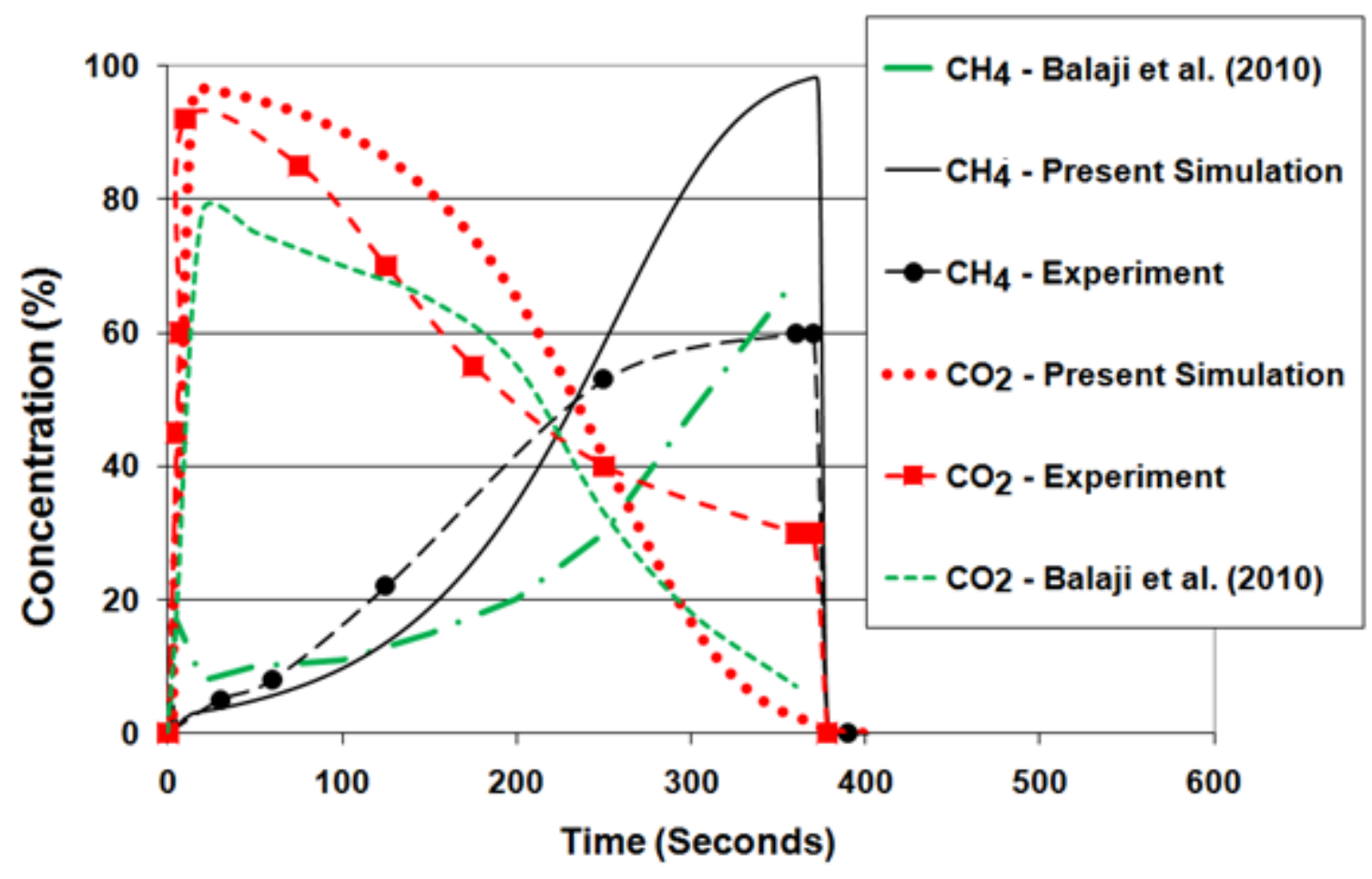

Fig. 7. 10: Outlet Flow concentration from simulations ignoring the formation of wustite or FeO (Mattisson et al. experiments, 2001).

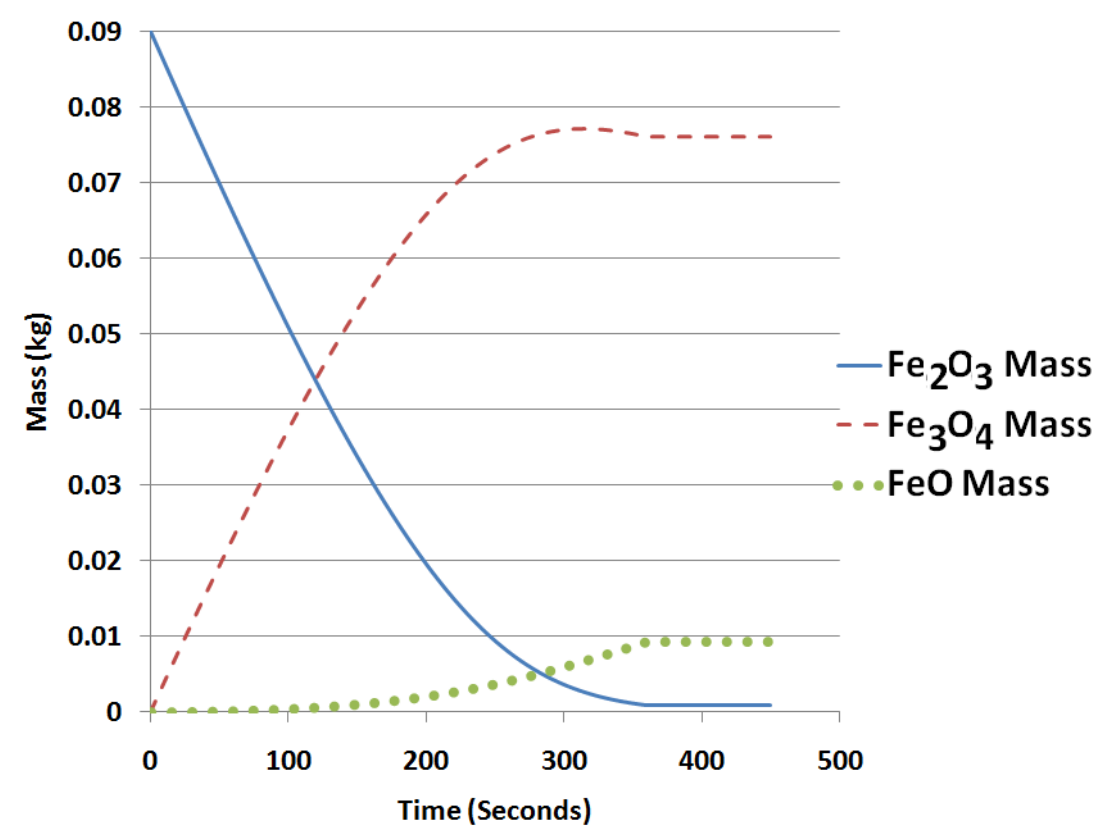

Fig. 7. 11: Time varying mass of hematite $\left(\mathrm{Fe}_{2} \mathrm{O}_{3}\right)$, magnetite $\left(\mathrm{Fe}_{3} \mathrm{O}_{4}\right)$ and wustite $(\mathrm{FeO})$ in the bed (Mattisson et al. experiments, 2001). 


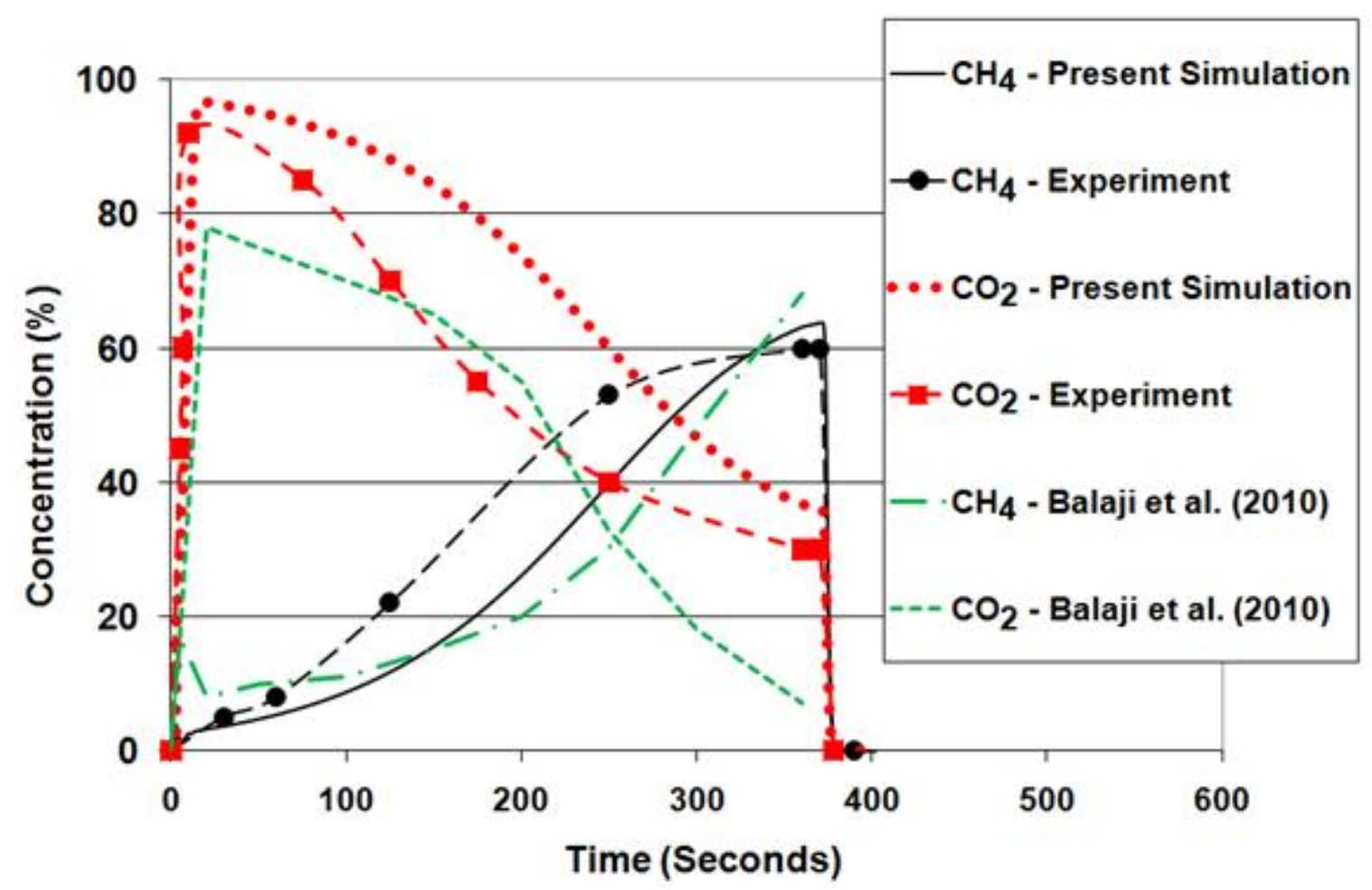

Fig. 7. 12: Outlet flow concentration from simulations including the formation of wustite (Experiments of Mattisson et al., 2001).

\subsection{Conclusion}

A CFD simulation model for methane combustion in the fuel reactor (FR) of chemical looping combustion (CLC) systems has been developed. The solid particles are modeled as a continuum fluid and appropriate equations have been used to model interactions between the solid particles. Detailed attention was paid to prepare a chemical kinetic model which accurately represented the experimental rates for heterogeneous reactions between the metal oxide particles and methane.

The CFD model was used to simulate two different sets of experiments. For the methane experiments of Son and Kim (2006) a good comparison with the experimental outlet concentration of flue gases was obtained. The CFD model was also found to be capable of capturing the changes in outlet concentration with change in superficial velocity, as well as changes in the reactor temperature and in the composition of the metal oxide itself. Also, bubble formation was observed in the reactor, and these bubbles were found to be regions of high fuel concentration. This could possibly be responsible for some part of the un-burnt fuel at the exit. The CFD model also gave a good comparison for the 75 
experiments of Mattisson et al. (2001). The numerically predicted time-varying concentration of flue gases matched well with experimental values. When the transformation of $\mathrm{Fe}_{3} \mathrm{O}_{4}$ to $\mathrm{FeO}$ was considered then the differences between predicted and experimental values were significantly reduced. 


\section{Chapter 8: Simulations of Batch Reactor for Coal Combustion}

Introduction

In the present chapter a CFD model has been developed for coal combustion in a fuel reactor of a $\mathrm{CL}$ combustor described in the literature which utilized an Fe-based carrier and a solid fuel, coal. Before a full scale coal CLC system is analyzed, it is important to make sure that the coal chemistry is mathematically modeled with reasonable accuracy. For this purpose a batch reactor where the complex effect of solid circulations is suppressed will be helpful. In a batch reactor the oxygen carrier particles are exposed to the alternating fuel and oxygen streams. A continuum model was used to describe both the gas and solid phases. Detailed sub-models to account for fluid-particle and particle-particle interaction forces were included. Global models of fuel and carrier chemistry were utilized. The results obtained from CFD were compared with detailed experimental time-varying outlet species concentrations, provided by Leion et al., 2008. The transient CFD simulations provided a reasonable match with the reported experimental data. For the present simulations the primary focus has been on the coal reactions during the reducing period of metal oxides.

\subsection{Details of Experimental Study}

The data to be simulated were provided by an experimental study of Leion et al. (2008) in which coal was consumed in a fluidized bed reactor. The operating gas velocity was above the minimum fluidizing velocity of the bed material. The fluidizing gas was $50 \%$ steam and $50 \% \mathrm{~N}_{2}$. Steam was introduced from the distributor, as part of the fluidization flow, to gasify the char remaining after the devolatilization process. The reactor was initially heated to the desired operating temperature ( $1223 \mathrm{~K}$ ) and then coal particles were released into the top section of the reactor. During the injection of coal, it was observed that some of the coal particles got stuck in the injector and some particles were elutriated due to disintegration. The estimated loss of coal was as high as $50 \%$ (Leion and Lyngfelt, 2009), however the exact amount lost is uncertain. In the present simulations the injected coal mass was taken to be $50 \%$ of that reported by Leion et al. (2008) which was the adjustment required so that the integrated total gas production agreed with the reported production. Steam was then introduced from the distributor, as part of the fluidization flow, to gasify the char remaining after the devolatilization process. The metal oxide used was illmenite $\left(\mathrm{Fe}_{2} \mathrm{O}_{3}\right.$ along with $\left.\mathrm{TiO}_{2}\right)$. Table 8.1 provides details of the Leion et al. (2008) experimental study. 
Table 8.1: Simulated Fuel Reactor Properties (Leion et al., 2007).

\begin{tabular}{|l|c|}
\hline Diameter of bed & $10-30 \mathrm{~mm}$ \\
\hline Flow rate at 0 oC and 1 atm & $600 \mathrm{ml} / \mathrm{min}$ \\
\hline Mass of metal oxide particles & $40 \mathrm{~g}$ \\
\hline Mass of coal particles & $0.1 \mathrm{~g}$ \\
\hline Mean diameter of metal particles & $105 \mu \mathrm{m}$ \\
\hline Mean diameter of coal particles & $155 \mu \mathrm{m}$ \\
\hline $\begin{array}{l}\text { Mean density of metal oxide particles } \\
\text { (including support) }\end{array}$ & $4500 \mathrm{~kg} / \mathrm{m}^{3}$ \\
\hline Initial density of coal particles & $2000 \mathrm{~kg} / \mathrm{m}^{3}$ \\
\hline Initial bed height & $35 \mathrm{~mm}$ \\
\hline Initial particle volume fraction & 0.6 \\
\hline Height of computational domain & $10 \mathrm{~m}$ \\
\hline
\end{tabular}

\subsection{The Reaction Scheme and Rates}

The following reaction mechanisms have been used:

Coal Devolatilization:

$$
\begin{aligned}
\text { Coal } \rightarrow & a C H A R+b \underset{\text { Soot }}{C}+c \mathrm{CH}_{4}+d C_{2} H_{6}+e C O+f \mathrm{CO}_{2}+g \mathrm{H}_{2} \\
& +h \mathrm{NH}_{3}+i \mathrm{H}_{2} \mathrm{O}+j \mathrm{H}_{2} \mathrm{~S}+k \mathrm{HCN}+l \mathrm{ASH}
\end{aligned}
$$

The coefficients (a through i) in Equation (8.1) have been determined from the ultimate and proximate analysis of the coal (Section 8.2.1 provides details).

Water-Gas-Shift Reaction:

$\mathrm{CO}+\mathrm{H}_{2} \mathrm{O} \rightarrow \mathrm{CO}_{2}+\mathrm{H}_{2}$ 


\section{Char Gasification by $\mathrm{CO}_{2}:$}

$$
\operatorname{Char}\left(\mathrm{CH}_{0.0245} \mathrm{O}_{0.00153} \mathrm{~N}_{0.0087} \mathrm{~S}_{0.0023}\right)+\mathrm{CO}_{2} \rightarrow 2 \mathrm{CO}+0.00842 \mathrm{H}_{2}+0.004375 \mathrm{~N}_{2}+0.001531 \mathrm{H}_{2} \mathrm{O}+0.0023 \mathrm{H}_{2} \mathrm{~S}
$$

Char Gasification by $\mathrm{H}_{2}$ O:

$\operatorname{Char}\left(\mathrm{CH}_{0.0245} \mathrm{O}_{0.001531} \mathrm{~N}_{0.00875} \mathrm{~S}_{0.0023}\right)+\mathrm{H}_{2} \mathrm{O} \rightarrow \mathrm{CO}+1.00842 \mathrm{H}_{2}+0.004375 \mathrm{~N}_{2}+0.001531 \mathrm{H}_{2} \mathrm{O}+0.0023 \mathrm{H}_{2} \mathrm{~S}$

Metal Oxide Reduction:

$$
\begin{aligned}
& 3 \mathrm{Fe}_{2} \mathrm{O}_{3}+\mathrm{CO} \rightarrow 2 \mathrm{Fe}_{3} \mathrm{O}_{4}+\mathrm{CO}_{2} \\
& 3 \mathrm{Fe}_{2} \mathrm{O}_{3}+\mathrm{H}_{2} \rightarrow 2 \mathrm{Fe}_{3} \mathrm{O}_{4}+\mathrm{H}_{2} \mathrm{O} \\
& 12 \mathrm{Fe}_{2} \mathrm{O}_{3}+\mathrm{CH}_{4} \rightarrow 8 \mathrm{Fe}_{3} \mathrm{O}_{4}+2 \mathrm{H}_{2} \mathrm{O}+\mathrm{CO}_{2} \\
& 21 \mathrm{Fe}_{2} \mathrm{O}_{3}+\mathrm{C}_{2} \mathrm{H}_{6} \rightarrow 14 \mathrm{Fe}_{3} \mathrm{O}_{4}+3 \mathrm{H}_{2} \mathrm{O}+2 \mathrm{CO}_{2}
\end{aligned}
$$

\subsubsection{Details of the Devolatilization Scheme:}

The model by Bradley et al., 2006 and Merrick, 1983, has been used to determine the components of primary devolatilization. Merrick, 1983, proposes the following breakup of coal:

\section{Coal $\rightarrow$ Char + Tar + Volatile_Species}

The volatile species are assumed to consist of $\mathrm{CH}_{4}, \mathrm{C}_{2} \mathrm{H}_{6}, \mathrm{CO}, \mathrm{CO}_{2}, \mathrm{H} 2, \mathrm{NH}_{3}, \mathrm{H}_{2} \mathrm{~S}$ and $\mathrm{H}_{2} \mathrm{O}$. Based on experimental studies of five different coals, Merrick proposes the mass fraction composition of char and tar as given in Table 8.2. Bradley et al. (2006) suggest that the decomposition of tar results in soot, $\mathrm{CH}_{4}$, $\mathrm{CO}, \mathrm{H}_{2}, \mathrm{H}_{2} \mathrm{~S}$ and $\mathrm{HCN}$. In the Leion et al. (2008) experiments, the rate controlling step is the gasification of char. The timescale for gasification of coal is usually several minutes while the devolatilization occurs in a matter of seconds. Because of the fast nature of devolatilization the primary and secondary devolatilization reactions have been combined to obtain Equation (8.1).

Table 8.2: Mass fraction of different elements in char and tar.

\begin{tabular}{|l|r|r|r|r|r|}
\hline & C & H & \multicolumn{1}{l|}{ O } & N & \multicolumn{1}{l|}{ S } \\
\hline Char & 0.98 & 0.002 & 0.002 & 0.01 & 0.006 \\
\hline Tar & 0.85 & 0.082 & 0.049 & 0.009 & 0.01 \\
\hline
\end{tabular}


The following has also been assumed (Bradley et al., 2006 and Merrick, 1983)

1) Methane consists of $32.7 \%$ of hydrogen in coal.

2) $\mathrm{C}_{2} \mathrm{H}_{6}$ consists of $4.4 \%$ of hydrogen in coal.

3) CO consists of $18.5 \%$ of oxygen in coal.

4) $\mathrm{CO}_{2}$ consists of $11 \%$ of oxygen in coal.

Based on the above assumptions coefficients for reaction 8.1 can be calculated. Appendix 1 provides the details. According to Bradley et al., 2006 the devolatilization can be expressed in the following form:

$\frac{d W_{k}}{d t}=k_{v, k}\left(W_{o k}-W_{k}\right)$

where $W_{o k}$ is the ultimate yield of the volatiles and $W_{k}$ is the mass already evolved. The rate used is $k_{V}=1 \times 10^{5} \exp \left(-12000 / T_{p}\right)$ from Bradley, 2006. This results in a devolatilization time scale of around a second at $1000 \mathrm{~K}\left(\mathrm{k}_{\mathrm{v}}=0.6141 / \mathrm{sec}\right)$. The calculated stoichiometric coefficients using the devolatilization model for South African coal used in the Leion, 2009 experiments are given in Table 8.3.

Table 8.3: Stoichiometric coefficients for devolatization.

\begin{tabular}{|c|c|r|}
\hline species & Label (Eq. 8.1) & \multicolumn{1}{c|}{ value } \\
\hline $\mathrm{C}$ & $\mathrm{a}$ & 0.4639 \\
\hline Soot & $\mathrm{b}$ & 0.012 \\
\hline $\mathrm{CH}_{4}$ & $\mathrm{c}$ & 0.0309 \\
\hline $\mathrm{C}_{2} \mathrm{H}_{6}$ & d & 0.0026 \\
\hline $\mathrm{CO}$ & e & 0.0096 \\
\hline $\mathrm{CO}_{2}$ & $\mathrm{f}$ & 0.00266 \\
\hline $\mathrm{H}_{2}$ & g & 0.0582 \\
\hline $\mathrm{NH}_{3}$ & $\mathrm{~h}$ & 0.0059 \\
\hline $\mathrm{H}_{2} \mathrm{O}$ & $\mathrm{i}$ & 0.0805 \\
\hline $\mathrm{H}_{2} \mathrm{~S}$ & j & 0.0011 \\
\hline $\mathrm{HCN}$ & k & 0.00013 \\
\hline $\mathrm{Ash}$ & $\mathrm{I}$ & 0.0165 \\
\hline
\end{tabular}

\subsection{Gasification Rates}

The expressions and constants for the gasification rates are obtained from Everson et al. (2006). For a single char particle, according to the shrinking core model (Szekely et al., 1976), 


$$
d X / d t=k(1-X)^{2 / 3} ; \quad \text { where, } k=\frac{S_{o}}{1-\varepsilon_{0}} r_{1}
$$

This implies the reaction rate $\left(\mathrm{kg} /\left(\mathrm{m}^{3} \cdot \mathrm{s}\right)\right)$ is:

$$
\dot{m}_{\text {char }}=\rho_{\text {char }} \varepsilon_{\text {char }} \frac{S_{o}}{1-\varepsilon_{0}} r_{1}(1-X)^{2 / 3}
$$

Here $X$ is the conversion, $S_{o}$ is the initial calculated particle surface area and $\varepsilon_{0}$ is the initial porosity.

The ratio was specified as

$$
\frac{S_{o}}{1-\varepsilon_{0}}=8.83 \times 10^{4} \mathrm{~m}^{2} / \mathrm{m}^{3}
$$

For char gasification by $\mathrm{H}_{2} \mathrm{O}, \mathrm{r}_{1}$ is given as

$$
r_{1}=r_{\mathrm{H}_{2} \mathrm{O}}=\frac{k_{\mathrm{H}_{2} \mathrm{O}} K_{\mathrm{H}_{2} \mathrm{O}} P_{\mathrm{H}_{2} \mathrm{O}}}{1+K_{\mathrm{H}_{2} \mathrm{O}} P_{\mathrm{H}_{2} \mathrm{O}}+K_{\mathrm{H}_{2}} P_{\mathrm{H}_{2}}}
$$

A similar expression has been used to calculate the $\mathrm{CO}_{2}$-char gasification rate, mutatis mutandis. The coefficients for the two gasification rates are listed in Table 8.4. Liu and Niksa (2004) provide a similar gasification model based on Langmuir-Hinshelwood rate expressions and suggest that the gasification rates for different types of coal can vary significantly and therefore there is a need for tuning of the gasification rates at one temperature. In the present simulations the rates for the South African coal were tuned at the specific operating temperature of $1223 \mathrm{~K}$, i.e., coefficients $\mathrm{k}_{\mathrm{CO} 2}$ and $\mathrm{k}_{\mathrm{H} 2 \mathrm{O}}$ were increased by a factor of 2 in comparison with that provided by Everson et al. (2006) in order to match

\begin{tabular}{|c|c|c|}
\hline & $\begin{array}{l}\text { Pre-exponential } \\
\text { Factor }\end{array}$ & Exponential Factor \\
\hline $\mathrm{k}_{\mathrm{CO} 2}$ & $* 3.96 \times 10^{-4} 1 / \mathrm{s}$ & $109 \mathrm{~kJ} / \mathrm{mol}$ \\
\hline $\mathrm{K}_{\mathrm{CO} 2}$ & $8.37 \times 10^{-5} 1 / \mathrm{Pa}$ & $16 \mathrm{~kJ} / \mathrm{mol}$ \\
\hline $\mathrm{K}_{\mathrm{CO}}$ & $1.9 \times 10^{-5} 1 / \mathrm{Pa}$ & - \\
\hline $\mathrm{k}_{\mathrm{H} 2 \mathrm{O}}$ & $* 22.1 / \mathrm{s}$ & $212 \mathrm{~kJ} / \mathrm{mol}$ \\
\hline $\mathrm{K}_{\mathrm{H} 2 \mathrm{O}}$ & $9.54 \times 10^{-2} 1 / \mathrm{Pa}$ & $69 \mathrm{~kJ} / \mathrm{mol}$ \\
\hline $\mathrm{K}_{\mathrm{H} 2}$ & $9.36 \times 10^{-5} 1 / \mathrm{Pa}$ & \\
\hline
\end{tabular}
experiments at this temperature. These rate coefficients (see Table 8.4) were used to simulate the reactor at all other operating conditions.

Table 8.4: Numerical values for coefficients in gasification reactions (Everson et al., 2006).

* increased by a factor of 2 from values provided above. 


\subsection{Water-Gas-Shift Reaction Rates (Bustamante et al., 2004)}

The reaction is given as:

$\mathrm{CO}+\mathrm{H}_{2} \mathrm{O} \rightarrow \mathrm{CO}_{2}+\mathrm{H}_{2}$

Bustamante et al. (2004) studied the reverse water gas-shift reaction and reported the reverse reaction rate and equilibrium constant. This reverse reaction rate was given in the form:

$$
\begin{aligned}
& r\left(k m o l /\left(m^{3} \cdot s\right)\right)=d[C O] / d t=k_{o} e^{-E / R T}\left[\mathrm{H}_{2}\right]^{\alpha}\left[\mathrm{CO}_{2}\right]^{\beta} \\
& K_{e q}=\exp (-4.33+4577.8 / T)
\end{aligned}
$$

The forward rate is calculated from the backward rate using the equilibrium constant. The net reaction rate is modified to be of the form:

$$
r=d[C O] / d t=-k_{o} \times\left(e^{-E / R T}\left[\mathrm{H}_{2}\right]^{\alpha}\left[\mathrm{CO}_{2}\right]^{\beta}-\frac{1}{K_{e q}} e^{-E / R T}\left[\mathrm{H}_{2} \mathrm{O}\right][\mathrm{CO}]\right)\left(\frac{k m o l}{m^{3} \mathrm{sec}}\right)
$$

The coefficients given by Bustamante et al. (2004) are provided in Table 8.5

\section{Table 8.5: Coefficients for water-gas-shift reaction rates}

\begin{tabular}{|l|r|}
\hline $\mathrm{k}_{\mathrm{o}}\left(\mathrm{m}^{3} / \mathrm{kmol}\right)^{\alpha+\beta-1} / \mathrm{s}$ & $2.17 \times 10^{+7}$ \\
\hline $\mathrm{E}(\mathrm{kJ} / \mathrm{mol})$ & 192.9 \\
\hline$\alpha$ & $1 / 2$ \\
\hline$\beta$ & 1 \\
\hline
\end{tabular}

\subsection{Metal Oxide Reaction Rates}

The iron oxide (hematite) reduction rates for $\mathrm{CO}$ and $\mathrm{H}_{2}$ were obtained from Mattisson et al. (2005). The reduction rate for $\mathrm{CH}_{4}$ was obtained from Mattisson et al. (2001). These reaction rates were determined by TGA. Since the experiments utilized a large excess of hematite, it was assumed that only reduction to magnetite need be included in the chemistry scheme so a simple one-step chemical reaction was assumed, as given by Equation (8.5). Experimental studies by Ryu et al. (2001) and Mattisson et al. (2005) suggest that the porous grain model (Szekely et al., 1976) can be used to explain the progress of these heterogeneous reactions. They also suggest that the outer boundary layer 
diffusion and pore diffusion of gases are fast and can therefore be neglected. The controlling step in these heterogeneous reactions is the slow chemical rates on the surface of the grains. The progress of reactions can be described by (Mattisson et al., 2005)

$\frac{t}{\tau}=1-(1-X)^{1 / 3}$ or $d X / d t=k(1-X)^{2 / 3}$

where $X=\frac{m-m_{r e d}}{m_{o x}-m_{r e d}}$.

Here $m_{o x}$ is the mass when the particle is fully oxidized and $m_{\text {red }}$ is the mass when particle is fully reduced. Also $R_{o}=\left(m_{o x}-m_{\text {red }}\right) / m_{o x}$.

Differentiating equation 8.16 yields:

$\frac{d X}{d t}=\frac{1}{m_{o x}-m_{r e d}} \frac{d m}{d t}=\frac{1}{R_{o} m_{o x}} \frac{d m}{d t}$

All the loss in mass of metal oxide is because of loss of oxygen. Therefore,

$d m=d m_{o 2}=M W_{o 2} \times d n_{O 2}$

Using above information the final rates are given as $\left(\mathrm{kg} / \mathrm{m}^{3} / \mathrm{s}\right)$

Reaction Rate for CO:

$\stackrel{*}{m_{\mathrm{CO}}}=\frac{k_{\mathrm{CO}} R_{o}}{2 M W_{\mathrm{O}_{2}}} \rho_{\mathrm{avg}} \varepsilon_{s}\left(Y_{\mathrm{Fe}_{2} \mathrm{O}_{3}}+Y_{\mathrm{Fe}_{3} \mathrm{O}_{4}} \times \frac{v_{\mathrm{Fe}_{2} \mathrm{O}_{3}} M W_{\mathrm{Fe}_{2} \mathrm{O}_{3}}}{v_{\mathrm{Fe}_{3} \mathrm{O}_{4}} M W_{\mathrm{Fe}_{3} \mathrm{O}_{4}}}\right)(1-X)^{2 / 3} M W_{C O}$

where $k_{C O}=\frac{3 b k\left(C_{C O}-C_{C O, e q}\right)}{\rho_{m} r_{o}} ; k=k_{o} e^{-E / R T}, k_{o}=6.2 \times 10^{-4} 1 / \mathrm{s}$ and $E=20 \mathrm{~kJ} / \mathrm{mol}$

\section{Reaction Rate for $\mathrm{H}_{2}$ :}

$\dot{m}_{\mathrm{H}_{2}}=\frac{k_{\mathrm{H}_{2}} R_{o}}{2 M W_{\mathrm{O}_{2}}} \rho_{\mathrm{avg}} \varepsilon_{s}\left(Y_{\mathrm{Fe}_{2} \mathrm{O}_{3}}+Y_{\mathrm{Fe}_{3} \mathrm{O}_{4}} \times \frac{v_{\mathrm{Fe}_{2} \mathrm{O}_{3}} M W_{\mathrm{Fe}_{2} \mathrm{O}_{3}}}{v_{\mathrm{Fe}_{3} \mathrm{O}_{4}} M W_{\mathrm{Fe}_{3} \mathrm{O}_{4}}}\right)(1-X)^{2 / 3} M W_{\mathrm{H}_{2}}$

where $k_{\mathrm{H}_{2}}=\frac{3 b k\left(C_{\mathrm{H}_{2}}-C_{\mathrm{H}_{2}, e q}\right)}{\rho_{m} r_{o}} ; k=k_{o} e^{-E / R T}, k_{o}=2.3 \times 10^{-3} 1 / \mathrm{s}$ and $E=24 \mathrm{~kJ} / \mathrm{mol}$. 
Reaction Rate for $\mathrm{CH}_{4}$ :

$\dot{m}_{C H_{4}}=\frac{k_{\mathrm{CH}_{4}} R_{o}}{2 M W_{\mathrm{O}_{2}}} \rho_{a v g} \varepsilon_{s}\left(Y_{\mathrm{Fe}_{2} \mathrm{O}_{3}}+Y_{\mathrm{Fe}_{3} \mathrm{O}_{4}} \times \frac{v_{\mathrm{Fe}_{2} \mathrm{O}_{3}} M W_{\mathrm{Fe}_{2} \mathrm{O}_{3}}}{V_{\mathrm{Fe}_{3} \mathrm{O}_{4}} M W_{\mathrm{Fe}_{3} \mathrm{O}_{4}}}\right)(1-X) \frac{Y_{\mathrm{CH}_{4}}}{Y_{\mathrm{CH}_{4}, T G A}} M W_{\mathrm{CH}_{4}}$

where, $k_{C_{4}}=5.33 \times 10^{-4} 1 / \mathrm{sec}$ and $Y_{C_{4}, T G A}=0.1$

The reaction rate is obtained from Mattisson et al., $2001 k_{C H_{4}}=0.032(1 / \mathrm{min})$. The rate for $\mathrm{CH}_{4}$ is formulated slightly differently as the coefficient ' $\mathrm{k}$ ' is given at one particular concentration of $\mathrm{CH}_{4}$.

The reaction rate for $\mathrm{C}_{2} \mathrm{H}_{6}$ was assumed to be the same as that for $\mathrm{CH}_{4}$ because of the unavailability of experimental data. The amount of $\mathrm{C}_{2} \mathrm{H}_{6}$ generated was small so that this is not a serious limitation.

\subsection{Simulating a Particle of Changing Size and Density}

In coal combustion the particle diameter and density vary with extent of conversion. At high temperatures $(T>1800 \mathrm{~K}$ ) the combustion reactions are so fast that reaction with surrounding gas takes place mainly at the outer surface and the density remains constant and the diameter changes with time (Smoot and Smith, 1985). At the low temperatures characteristic of fluidized beds ( $<1300 \mathrm{~K}$ ), because the gasification reactions are slow it is thought that the reacting gas has enough time to penetrate through the pores and the reactions take place throughout the volume of the particle (Syamlal and Bisset, 1992). Based on this reasoning, it has been assumed that the particle diameter is initially constant, but the density is a linear function of conversion, $X$ :

$$
\rho_{P}=\left\{\begin{array}{cl}
\rho_{P, \text { max }}(1-X) & X<X_{c} \\
\rho_{P, \text { min }} & X>X_{c}
\end{array}\right.
$$

The density of the unburned coal, $\rho_{P, \text { max }}$, is assumed to be $2000 \mathrm{~kg} / \mathrm{m}^{3}$. The density has been limited in the present study to a minimum value, $\rho_{P, \min }=100 \mathrm{~kg} / \mathrm{m}^{3}$.

\subsection{The Initial and Boundary Conditions}

The initial bed height of $35 \mathrm{~mm}$ (Table 8.1) was determined from the geometry of the reactor, the density of iron oxide and the total mass of iron-oxide initially in the bed. Further, the coal was assumed to be initially fully mixed with the iron oxide. In the experiments the entire reactor was placed 84 
in an oven at $950{ }^{\circ} \mathrm{C}$ (Leion et al., 2007). To simulate these conditions the walls of the reactor were assumed to be at $950{ }^{\circ} \mathrm{C}$ and the inlet gas was also assumed to be at this temperature.

\subsection{Numerical Parameters and Grid Convergence Study}

The CFD domain for the geometry of the FR is shown in Fig. 8.1. The grid used in the simulation was a 2-D, axisymmetric quadrilateral grid. In Fig. 8.1, for display purposes, the mesh has been mirrored about its axis. Two different grid sizes were used. The cell count in the fine mesh exceeds that in coarse mesh by a factor of 2.25 . The typical cell dimension at different regions of the coarse mesh is provided in Fig. 8.1. The time step size for the fine mesh was reduced by a factor of two for numerical stability. The discretization scheme and other numerical parameters are provided in Table 8.6. Fig. 8.2 compares the time varying outlet gas concentrations obtained from the coarse and fine mesh. A temperature of $950{ }^{\circ} \mathrm{C}$ and $50 \%$ inlet steam concentration was used for these simulations. The maximum difference in the gas concentration obtained from the coarse and fine mesh occurs at the peak concentration during devolatilization (Fig. 8.2). The maximum difference in the computed gas concentration is around $5 \%$, but at most times the error is significantly less. The coarse mesh was used in all subsequent simulations as the computational time required for the fine mesh is significantly larger.

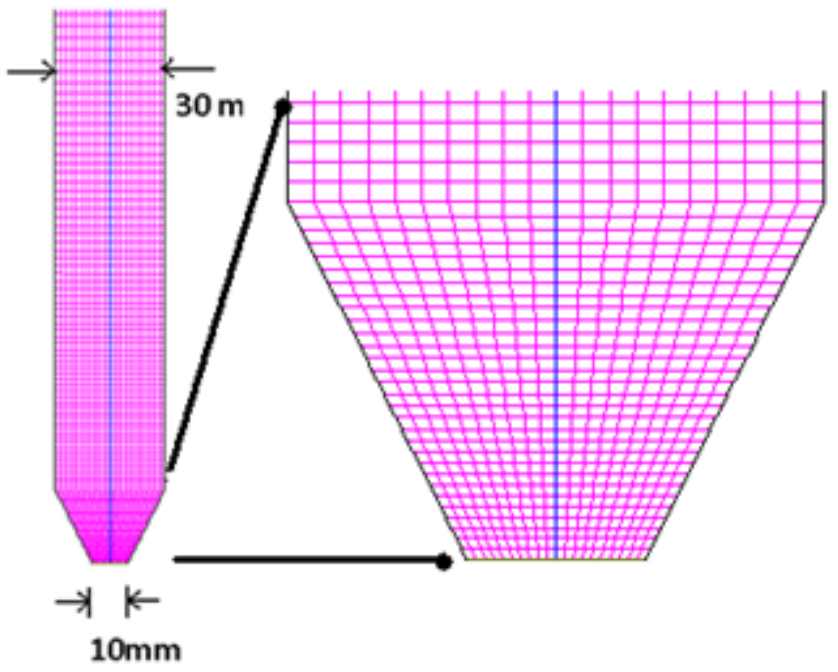

\begin{tabular}{|l|c|}
\hline \multicolumn{1}{|c|}{ Location } & $\begin{array}{c}\text { Cell } \\
\text { Dimension } \\
\text { (Coarse Mesh) }\end{array}$ \\
\hline Throat & $0.05 \mathrm{~cm}$ \\
\hline Top of expansion & $0.15 \mathrm{~cm}$ \\
\hline Above bed at 3m height & $4 \mathrm{~cm}$ \\
\hline
\end{tabular}

Fig. 8.1: The lower portion of the computational mesh. The mesh is mirrored about the axis. 
Table 8.6: Summary of numerical parameters.

\begin{tabular}{|c|c|c|}
\hline No. of cells & Coarse Mesh & Fine Mesh \\
\hline $\begin{array}{c}\text { Spatial discretization scheme for the } \\
\text { momentum equations }\end{array}$ & Second Order Upwind & Second Order Upwind \\
\hline $\begin{array}{c}\text { Spatial discretization scheme for each } \\
\text { phase and species equation }\end{array}$ & First Order Upwind & First Order Upwind \\
\hline Time integration scheme & First Order Implicit & First Order Implicit \\
\hline Time step size & 0.001 seconds & 0.0005 seconds \\
\hline Iterations per time step & 20 & 20 \\
\hline
\end{tabular}

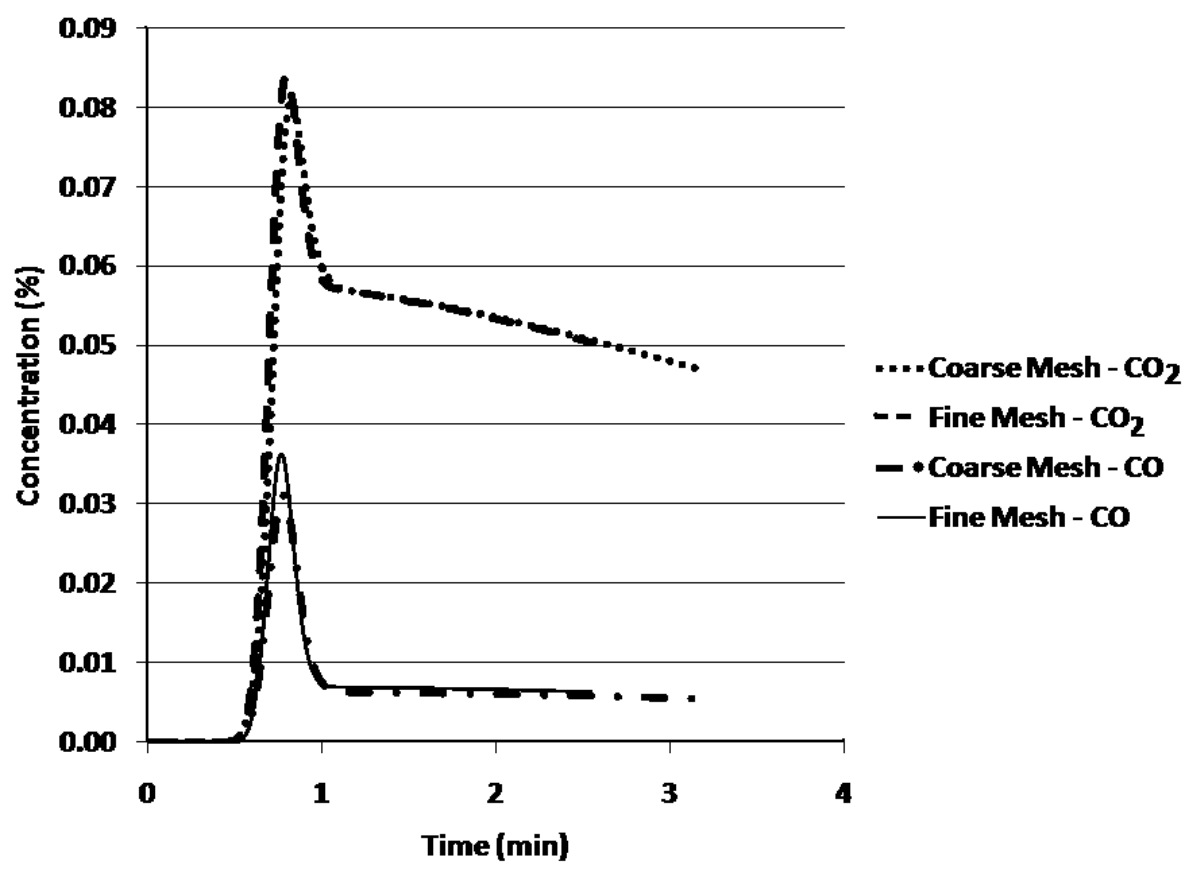

Fig. 8.2: Comparison of the calculated time development of the $\mathrm{CO}_{2}$ and $\mathrm{CO}$ concentrations using a coarse and a fine mesh (Temperature of $950{ }^{\circ} \mathrm{C}$ and $\mathrm{H}_{2} \mathrm{O}$ concentration of $50 \%$ ).

\subsection{Results}

Figs. 8.3 show instantaneous velocity vectors and volume fraction contour plots for both iron oxide and unreacted char, at approximately 175 seconds into the simulation. The time instant of 175 seconds has been chosen because the devolatilization products have left the system by this time and the 
rate constraining gasification reactions are dominant. (Since the experiments were "batch," timeaverage results, as are usually shown, have no real meaning.) The regions of low solids volume fraction (high gas volume fraction) display a "bubble". Bubbles allow gases to bypass the emulsion phase and, thus, can allow combustible gases to escape without reacting with the metal oxides. The velocity vectors plotted in Fig. 8.3a and Fig. 8.3b clearly show a high velocity central jet which causes the solids to spout. Comparing phase contour plots of metal oxide and coal (Fig. 8.3a and 8.3b) it is clearly observed that the region of large volume fraction of metal oxide is also the region of large volume fraction for coal indicating that both the coal and iron oxide particles remain well mixed during the simulation. Also the solids velocities are the same order of magnitude for both coal and metal oxide phases which further establishes the well mixed nature of the particles. The mixing of metal oxide and coal is essential for high efficiency of the reactor. If coal particles segregate in the upper portion of the bed due to their progressively lower density the $\mathrm{CO}$ and $\mathrm{H}_{2}$ released by gasification will not contact the carrier particles, resulting in partial combustion and, hence, a reduction in the combustion efficiency.

The bubbles are formed at the inlet and rise mainly along the central axis of the reactor and the particles move upward, dragged in their wake. This causes a higher concentration of solids, mainly iron oxides, returning in a down flow along the side walls of the reactor (Fig. 8.3a). Fig. 8.4a shows the mass fraction of steam, the primary gasifying agent, constituting $50 \%$ of the inlet gas as well as the gas phase velocity vectors. As expected the peak mass fraction of $\mathrm{H}_{2} \mathrm{O}$ occurs in the bubble regions. The drop in its gas mass fraction in the interstitial flow in the emulsion phase is due, in part, to gasification of coal. The gas velocity vectors show a high velocity in the bubble region indicating the rapid rise of the bubbles. Fig. 8.4b shows the mass fraction of $\mathrm{CO}_{2}$. It is high in the emulsion phase, where it is produced, and low in the bubble regions. Only a small amount of $\mathrm{CO}_{2}$ moves through the bubble. Fig. $8.4 \mathrm{c}$ shows the $\mathrm{H}_{2}$ mass fraction, a product of coal gasification. It is high in the emulsion region, where it is produced, and can further react with the carrier. Only a small amount of $\mathrm{H}_{2}$ is present in the bubble regions and hence it is likely that only a small percentage of $\mathrm{H}_{2}$ by-passes the bed through the bubble .

In the experiments the concentration of outlet flue gases was measured after 40 seconds of time delay (Leion et al., 2007). This was primarily the time required for gases to flow through a pipe connecting the reactor and gas analyzer. Experiments by Taylor (1954) demonstrate that there can be significant "apparent diffusion" of gases when they travel through pipes. This can lead to changes in peak gas concentrations. To account for this diffusion in the flow between reactor and gas analyzer, the 
length of the simulated reactor was extended to $10 \mathrm{~m}$ using an expanding mesh. In the simulations the outlet gas concentration has been measured at a height of $3 \mathrm{~m}$ (Fig. 8.5) to approximate the 40 seconds of time delay in the experimental measurements. Also, the diffusive effects damp out oscillations in concentration of flue gases that are calculated close to the top of the bed because of bubble eruptions.

The peak $\mathrm{CO}_{2}$ concentration predicted by the simulations agrees well with the experiments (Fig. 8.5 and Table 8.7). The computed char burn out time is close to the experimentally observed 15 minutes. The peak $\mathrm{CH}_{4}$ and $\mathrm{CO}$ concentration predicted by the simulations differs by $30 \%$ in comparison with experimental values. These differences may be attributed to the assumptions made in the modeling of the reactor as well as the uncertainty in the injection of coal mass as mentioned in section 8.1.

In the operation of the current CLC reactor, the gasification reaction is the slowest and it requires several minutes to completely gasify the coal. The gasification can occur through two reaction pathways as indicated by Equations (8.3) and (8.4). Fig. 8.6 shows the local char consumption rates $\left(\mathrm{kmol} / \mathrm{m}^{3} / \mathrm{s}\right)$ at 175 seconds due to gasification by both steam and $\mathrm{CO}_{2}$. Obviously, these rates are highest in regions of high solids concentration (the "emulsion phase"). The char consumption rate due to steam is at least 30 times higher than $\mathrm{CO}_{2}$ (Fig. 8.6). This is because (i) the concentration of steam is higher and (ii) the reaction rate for steam gasification is higher.

Fig. 8.7 shows the variation in the total hematite, magnetite and char mass during coal combustion. There is a rapid change in the mass at times close to zero. This is due to the rapid devolatilization of coal and the reaction of the volatiles with the metal oxide. Both hematite and magnetite reach a stable asymptotic value with further consumption of coal. Also hematite is present in excess amounts in the system and therefore the drop in mass of hematite is only around $20 \%$. Fig. 8.7 also shows the mass of char scaled by a factor of 100 . The initial rise in mass of char at times close to zero is because of its formation as a product of devolatilization. The further gradual decrease in its mass is due to gasification. Table 8.8 compares the mass of carbon input into the system and that eventually leaving it. The carbon mass balance is within $0.5 \%$. Part of this error is attributed to the time integration procedure (trapezoidal rule) used during post-processing to evaluate the total carbon mass flowing through the exit. 


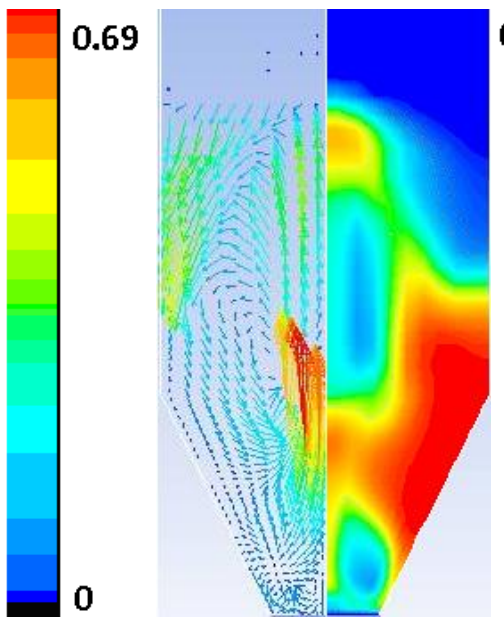

a) Iron oxide

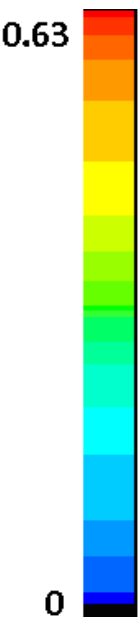

0
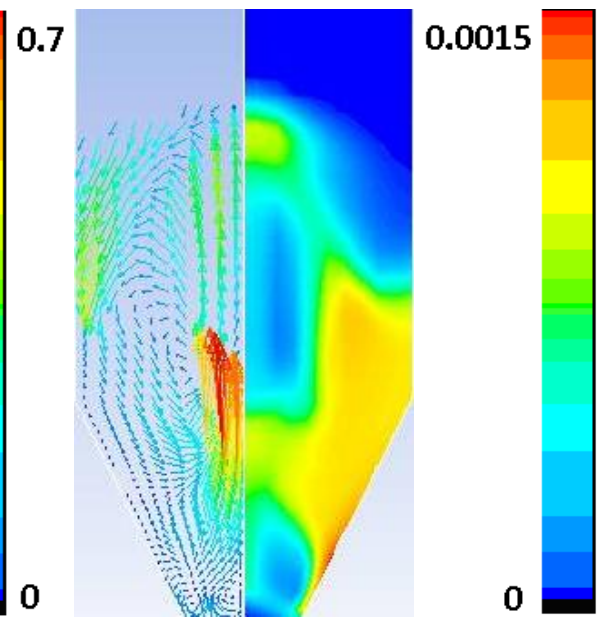

b) Coal

Fig. 8.3: Velocity vectors $(\mathrm{m} / \mathrm{s})$ and volume fractions of iron oxide and coal particles at 175 seconds, temperature of $1273 \mathrm{~K}$ and $50 \%$ steam inlet concentration.
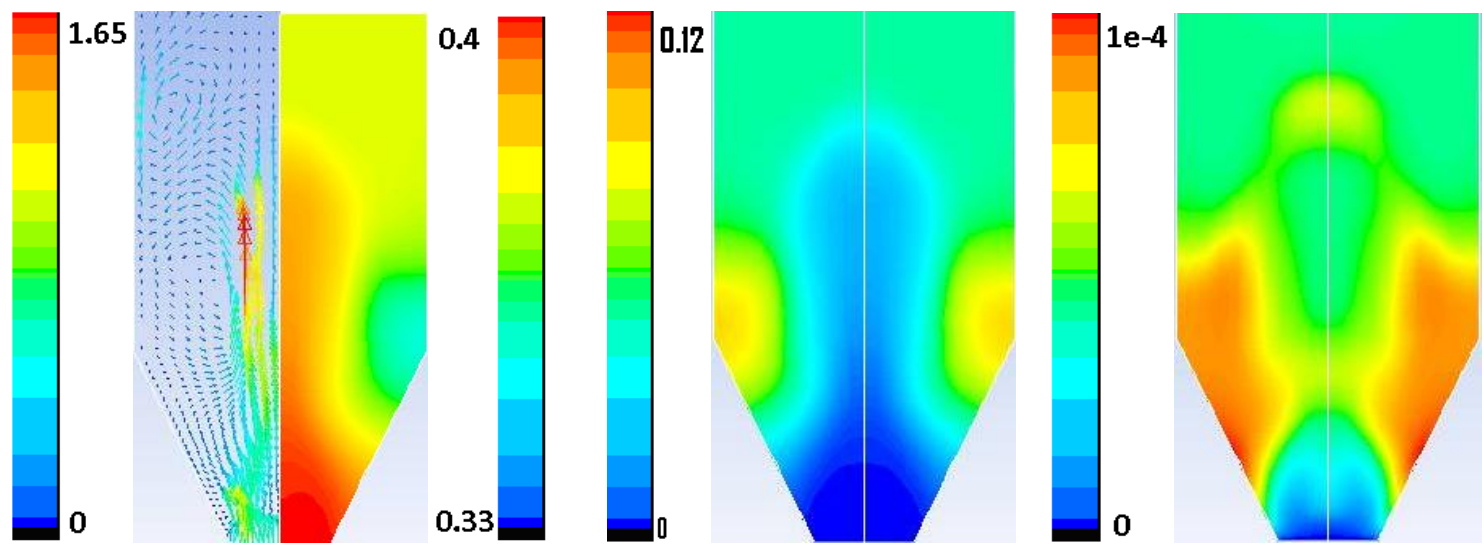

a) $\mathrm{H}_{2} \mathrm{O}$

b) $\mathrm{CO}_{2}$

c) $\mathrm{H}_{2}$

Fig. 8.4: Instantaneous gas velocity vectors (m/s) [Fig. 5a] and gas species mass fractions at 175 seconds, temperature of $1273 \mathrm{~K}$ and $50 \%$ steam inlet concentration.

Table 8.7: Peak Concentration from Experiment and Simulation.

\begin{tabular}{|l|r|r|}
\hline & Peak Concentration Experimental & Peak Concentration Simulation \\
\hline $\mathrm{CH}_{4}$ & 0.036 & 0.049 \\
\hline $\mathrm{CO}_{2}$ & 0.078 & 0.076 \\
\hline $\mathrm{CO}$ & 0.047 & 0.022 \\
\hline
\end{tabular}




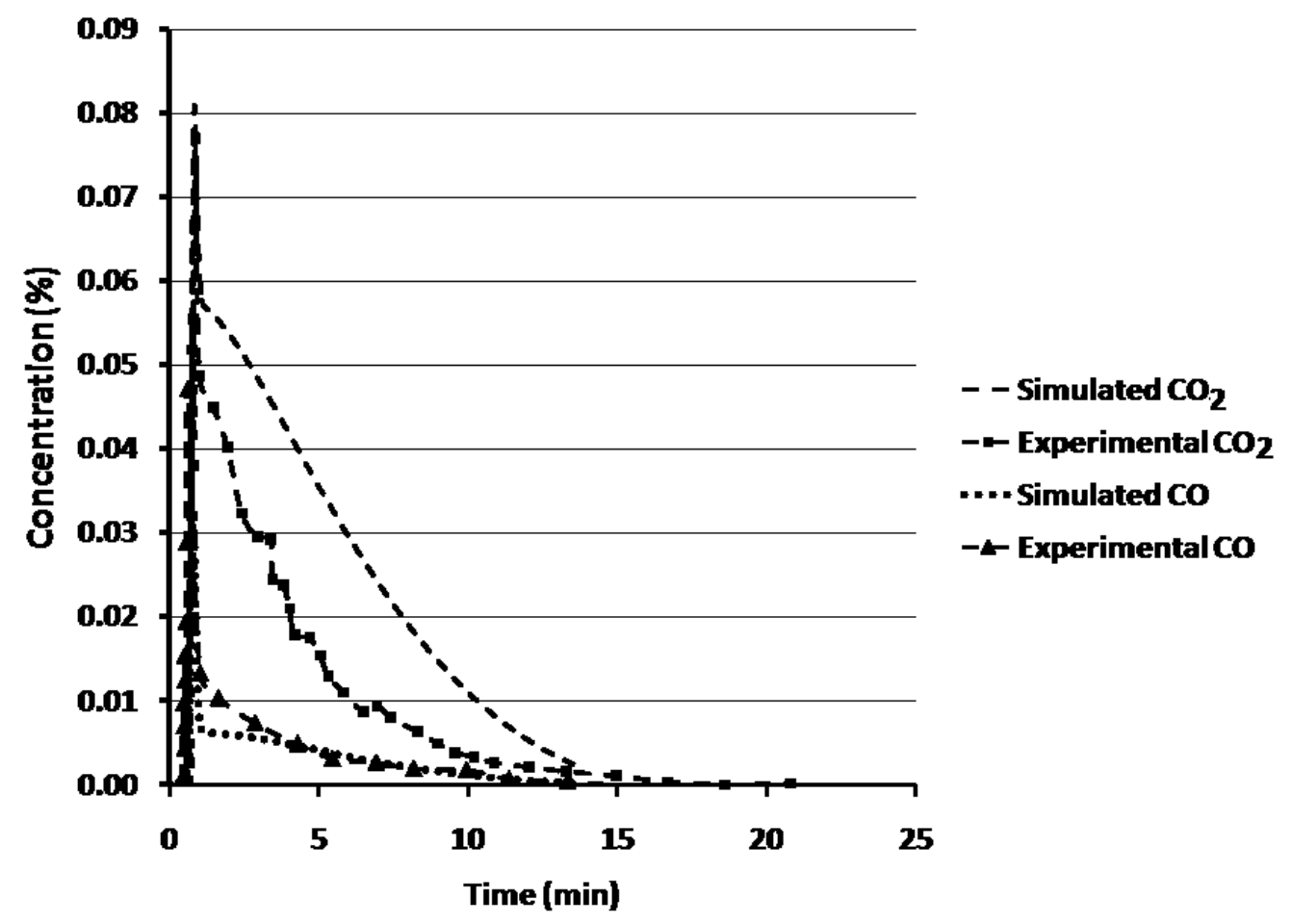

Fig. 8.5: Calculated concentrations of $\mathrm{CO}_{2}, \mathrm{CO}$ and $\mathrm{CH}_{4}$ at a height of $3 \mathrm{~m}$ compared to the experimental data of Leion et al., 2008. The temperature is $1223 \mathrm{~K}$ and $50 \%$ inlet steam concentration is used.

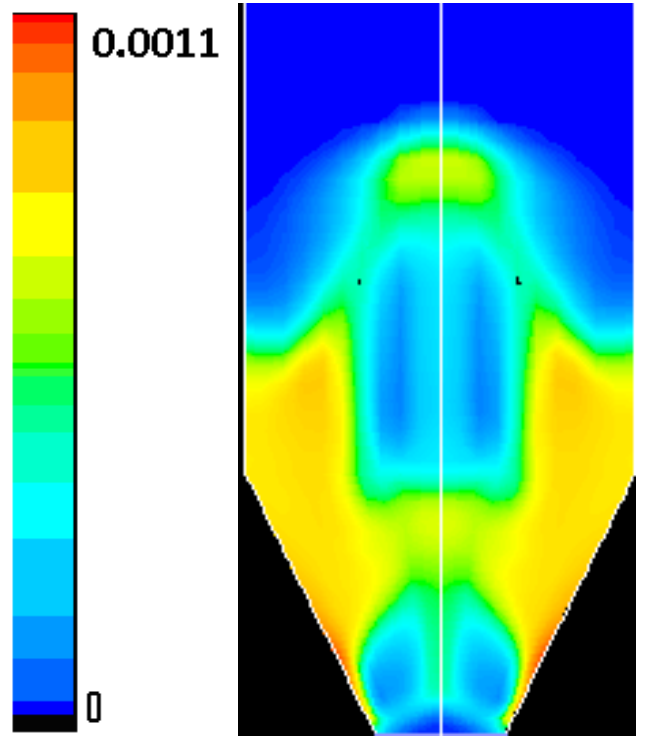

a) Steam gasification Rate

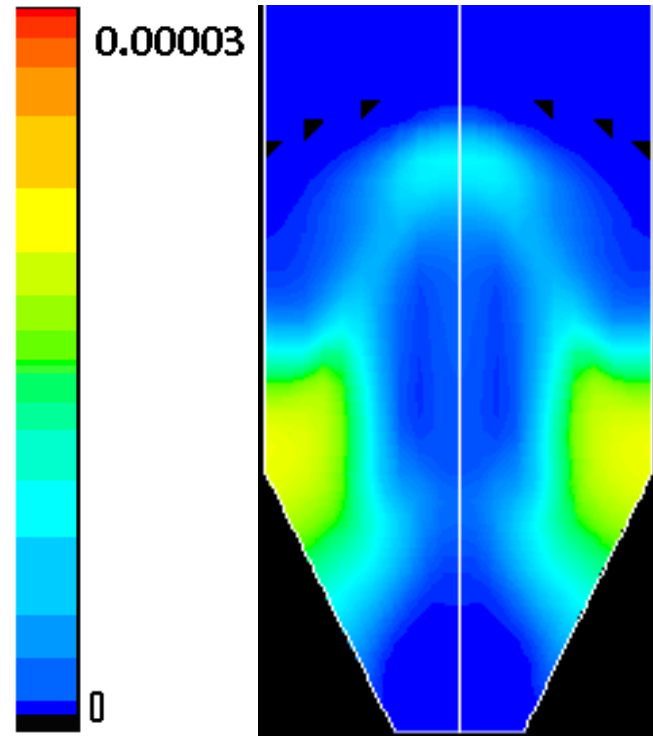

b) $\mathrm{CO}_{2}$ gasification rate

Fig. 8.6: Local gasification rates of char particles $\left(\mathrm{kmol} / \mathrm{m}^{3} / \mathrm{s}\right)$ at 175 seconds, temperature of $1273 \mathrm{~K}$ and $50 \%$ steam inlet concentration. 
Table 8.8: Computational mass balance of carbon in reactor.

\begin{tabular}{|l|l|l|l|}
\hline & $\begin{array}{l}\text { Total Mass Injected through } \\
\text { Coal }\end{array}$ & $\begin{array}{l}\text { Total mass of carbon exiting at } \\
3 \mathrm{~m} \text { as gases }\end{array}$ & \% Error \\
\hline Carbon & 0.125 gram (or 62.5\% of Coal) & $\begin{array}{l}0.12435 \text { gram } \\
\left(\mathrm{CO}_{2}+\mathrm{CO}+\mathrm{Soot}+\mathrm{CH}_{4}+\mathrm{C}_{2} \mathrm{H}_{6}\right)\end{array}$ & 0.52 \\
\hline
\end{tabular}

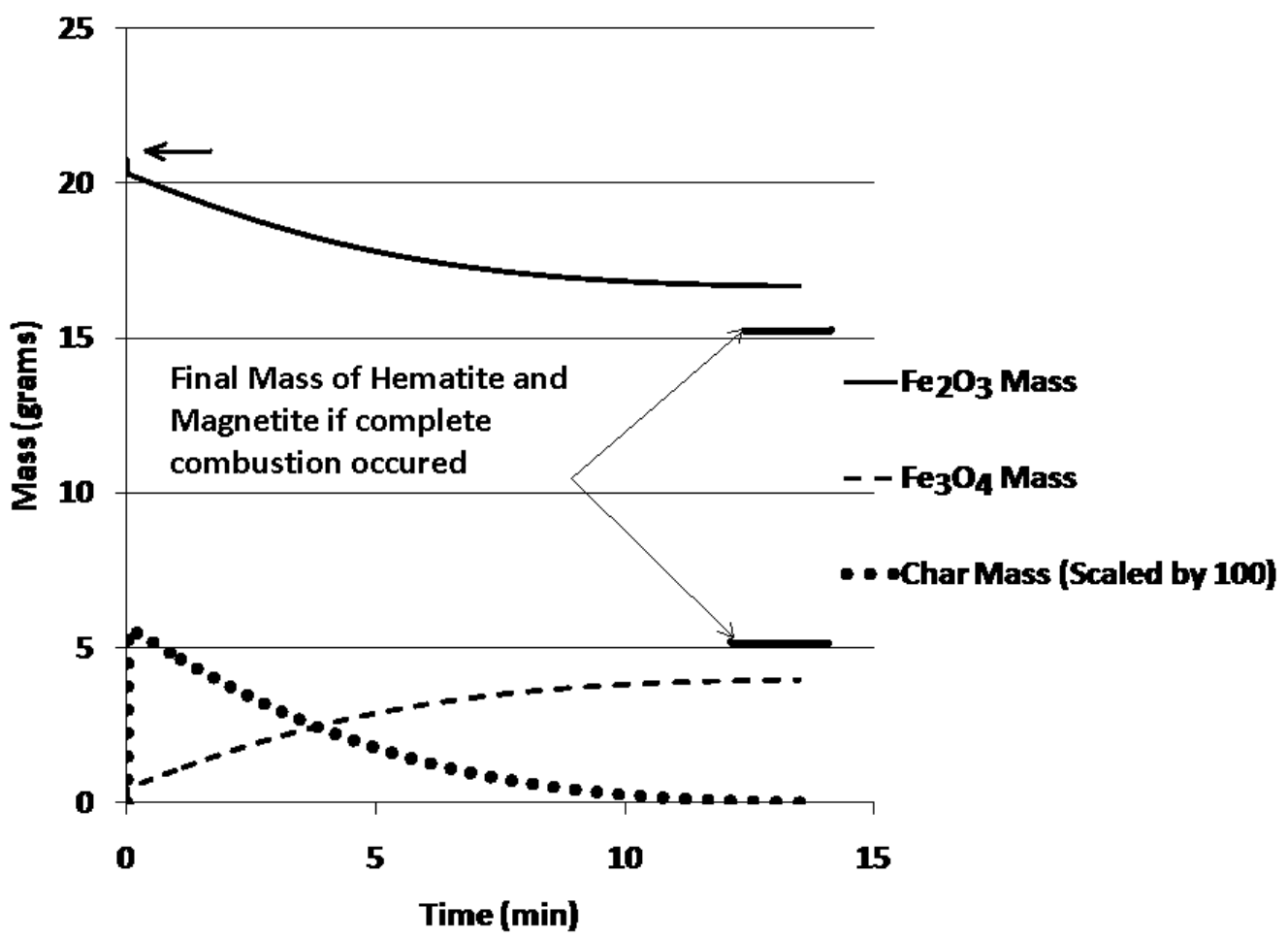

Fig. 8.7: Time variation of hematite $\left(\mathrm{Fe}_{2} \mathrm{O}_{3}\right)$ and magnetite $\left(\mathrm{Fe}_{3} \mathrm{O}_{4}\right)$ mass. Temperature of $1223 \mathrm{~K}$ and $50 \%$ steam concentration.

Effect of Higher Operating Temperature:

Fig. 8.8 shows the effect of operating temperature on the average coal reaction rates, defined as (Leion et al., 2008):

$R_{\text {avg }}=\frac{1}{m_{\text {tot }}} \frac{m_{t}}{t}$

where $m_{\text {tot }}$ is the total mass of carbon converted and $m_{t}$ is the total mass of carbon converted up until time $t$, the time from start of the experiment/simulation. The simulations are in agreement within 91 
experimental variations observed at most operating conditions showing rapid increase with increasing temperature. Recall that the Everson gasification rates had to be increased by a factor of two to obtain agreement at $950^{\circ} \mathrm{C}$. This was not further adjusted for the simulations at higher or lower temperatures; the rates increased due to their Arrhenius temperature dependence.

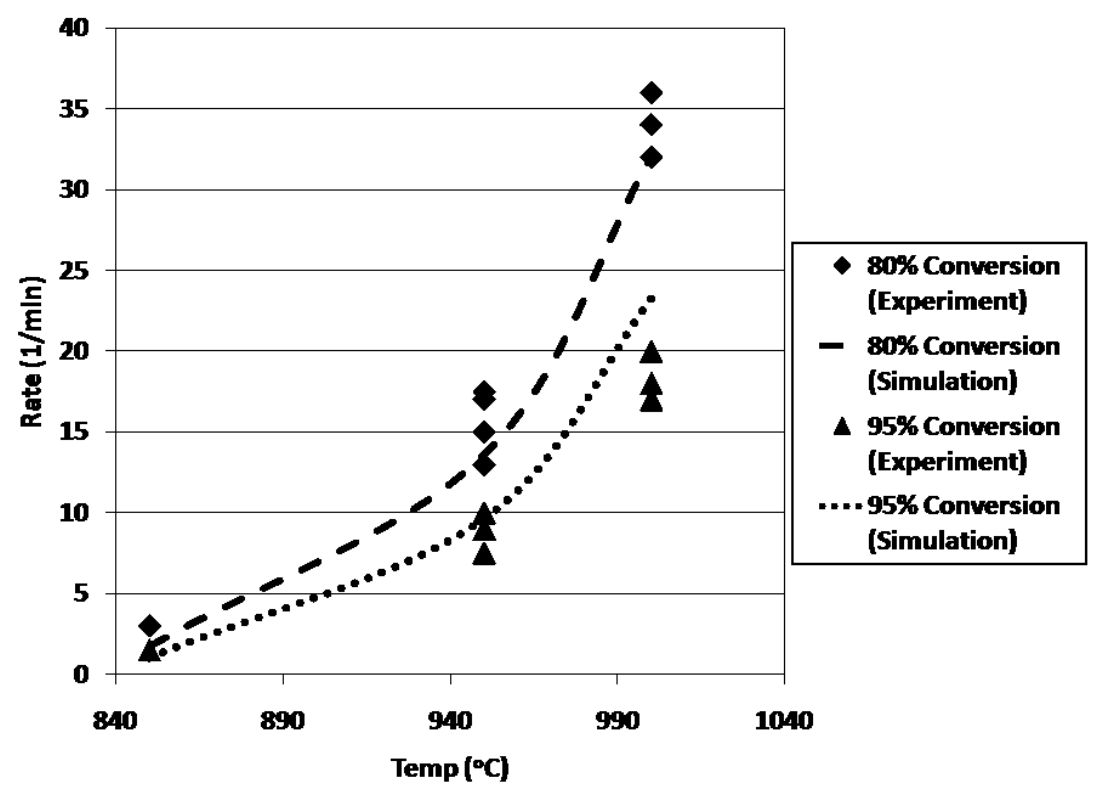

Fig. 8.8: The average coal reaction rate (see text) at different operating temperatures $(50 \%$ Steam concentration).

\section{Effect of steam concentration:}

The effect of the variation of steam concentration in the fluidization gas on the average reaction rates is shown in Fig. 8.9. Once again the simulation results are generally within the observed experimental variations and predict the slight increase in reaction rate with increasing $\mathrm{H}_{2} \mathrm{O}$ concentration due to increased rate of char gasification. 


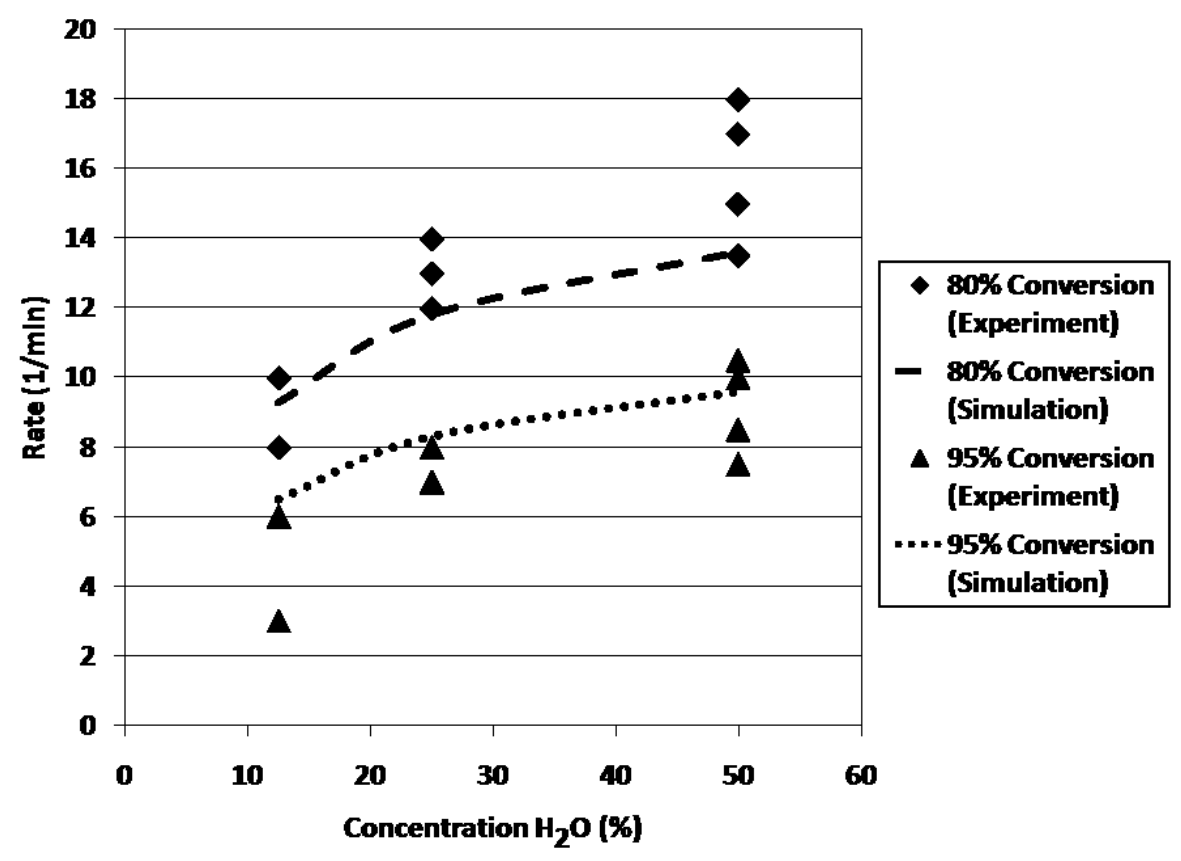

Fig. 8.9: The average coal reaction rate (see text) at different steam concentration, temperature of 1223K.

\subsection{0: Conclusion}

A CFD model for simulation of coal combustion in the fuel reactor of chemical looping combustion systems has been developed. The solid particles are modeled as an interpenetrating continuum fluid. Chemical kinetic models have been assembled from the literature for the reactions between the iron oxide and fuel gases as well as the devolatilization and char gasification of coal. The CFD model has been used to simulate the experiments of Leion et al. (2008). The model was able to predict the transient outlet concentrations of $\mathrm{CO}_{2}, \mathrm{CO}$ and $\mathrm{CH}_{4}$ reasonably well. The reactor performance at different operating temperatures and inlet steam concentrations was also captured in a reasonable manner. This demonstrates that CFD modeling can be an effective approach in the design of such a reactor. 


\section{Chapter 9: Simulations of Full Circulating Fluidized Bed CLC Systems}

\section{Introduction}

In the present chapter CFD simulations of a full circulating fluidized bed have been carried out and validated with experimental data. As discussed in previous chapters, CLC requires many unit operations involving gas-solid or granular flow. A CLC system generally consists of two reactors, a Fuel Reactor (FR) and an Air Reactor (AR). The fuel is oxidized in the FR by using granular metal oxides. The FR is typically a bubbling or moving bed. The AR re-oxidizes the reduced carrier and is typically a transport reactor. At its exit of $A R$ the oxidized carrier is separated by a cyclone or an expansion region and returned to the FR. Thus the CLC reactor basically is a circulating fluidized bed (CFB) where solid particles are circulated between the AR and FR.

To date very limited CFD simulations have been performed on full circulating fluidized beds particularly due to the complexities in geometry, the flow physics and the large computational capacity required to simulate them. Also many CFD simulations limit themselves to the riser section of the CFB (O'Brien and Syamlal, 1993, Guenther et al., 2002, Mao et al., 2004). Simulating a limited section of the CFB will require inputs to the numerical model that can be difficult to ascertain in an accurate manner (such as the solids circulation rates). Simulating individual units of a CFB system can therefore to some extent limit the use of CFD in a design study. Also, the advanced CFB systems that are being developed today (such as CLC) will require the use of complex control systems to run them in an efficient manner. To design these control systems there is a need to develop mathematical models that can predict the behavior of the entire CFB in an accurate manner. Once validated for a particular application, CFD can be used as a tool to develop these complex mathematical models or it can be directly coupled with the control system design software. Simulations of a full circulating fluidized bed have been performed by Samuelsberg and Hjertager (1996); and Mathiesen et al., (2000). However in their two dimensional CFD simulations they simplified the complex three dimensional experimental geometry into two dimensions and primarily focused on comparing results within the riser section. In most CFB systems the flow of solids is mostly limited by narrow flow sections such as pipes, loop seals etc and hence there is a strong need to accurately represent these regions in a CFD simulation. In a CLC system predicting the right solid circulation rates for an oxygen carrier is essential as it will directly affect the amount of fuel that can be burnt and also effect the heat transfer between reactors. In the present study detailed mathematical 94 
models have been incorporated to describe the flow of solid particles both in the dense and dilute regimes so that an entire CFB CLC system can be analyzed.

The design of a chemical looping combustion system requires that a number of important parameters be considered, including:

- Combustion efficiency of the reactor,

- Carbon capture efficiency of the reactor,

- Mass distribution of metal oxides in the air and fuel reactors,

- Solid circulation rate to provide enough oxygen for combustion of fuel and heat balance between reactors, and

- Gas leakage between reactors which can result in lower carbon capture efficiency and dilution of the $\mathrm{CO}_{2}$ concentration in the fuel reactor exhaust gases.

The use of CFD can help in estimating the performance of the reactor based on the above parameters without making detailed assumptions in the modeling procedure. The focus in this study is on accurately predicting the performance of a complete experimental CLC system designed by Kronberger et al., (2004) and Abad et al. (2006). The Kronberger et al. (2004) and Abad et al. (2006) experiments were selected for a coupled air and fuel reactor simulations because of following reasons:

1) The geometry can easily be approximated as $2 D$ since the depth is constant.

2) Kronberger et al. evaluates only the fluid mechanics and ignores the chemistry. Predicting the solid circulation rates and flow patterns are the essential first step in simulations of a complete CLC system.

3) Hot circulating CLC data with gaseous fuel from Abad et al. (2006) is available on a very similar geometry setup as that of Kronberger et al.

4) It is one of the few complete CLC experiments. Most CLC studies are either based on fluidized or fixed bed which is alternated as fuel and air reactor and hence are not good approximations of the air reactor.

The present transient CFD simulations provided a reasonable match with the reported experimental data. 


\subsection{Details of Kronberger et al. (2004) experimental study}

The experiments are for a cold flow reactor which consists of FCC particles that are fluidized by a combination of helium and nitrogen. The air reactor fluidizing velocity is higher than the terminal velocity of the particles and therefore caries the particles upwards. At the top section there is a sudden expansion of flow due to the presence of a particle separator, and this causes the particles to fall back down. In the experiments the particle separator consists of an expansion perpendicular to the plane of the paper. Since the simulations are assuming a two-dimensional domain, this expansion has been modified such that it occurs in the plane of the paper (Figs. 9.1a and 9.1b). Table 9.1 provides details of the experiment. Since this expansion occurs downstream of the circulating bed it is not likely to affect the solution significantly.

Table 9. 1: System properties for experiments of Kronberger et al. (2004).

\begin{tabular}{|l|l|}
\hline Width of Fuel Reactor (mm) & 19 \\
\hline Width of Air Reactor (mm) & 27 \\
\hline Width of Downcommer (mm) & 9 \\
\hline $\begin{array}{l}\text { Fluidizing gas composition in air and fuel } \\
\text { reactor (N2/He\%) }\end{array}$ & $13 / 87$ \\
\hline Average diameter of particles (microns) & 70 \\
\hline Average density of particles (kg/m ${ }^{3}$ ) & 1500 \\
\hline $\begin{array}{l}\text { Minimum Fluidization velocity of particle } \\
\text { (Wu and Yen, 1966) (m/s) }\end{array}$ & 0.0027 \\
\hline Terminal velocity of particle (m/s) & 0.2 \\
\hline Range of Fuel Reactor velocity & 18 to 55 times minimum fluidization velocity \\
\hline & 1.2 to 2 times terminal velocity \\
\hline Range of Air Reactor velocity & 43 to $58 \mathrm{~g}$ \\
\hline Mass of metal oxide particle bed (g)
\end{tabular}

\subsubsection{Details of Numerics for Kronberger experiments}

The CFD mesh used in the simulations is shown in Fig. 9.2. The grid used in the simulation was a quadrilateral cell grid. Table 9.2 provides details about the grid size and numerics used in the simulations. 


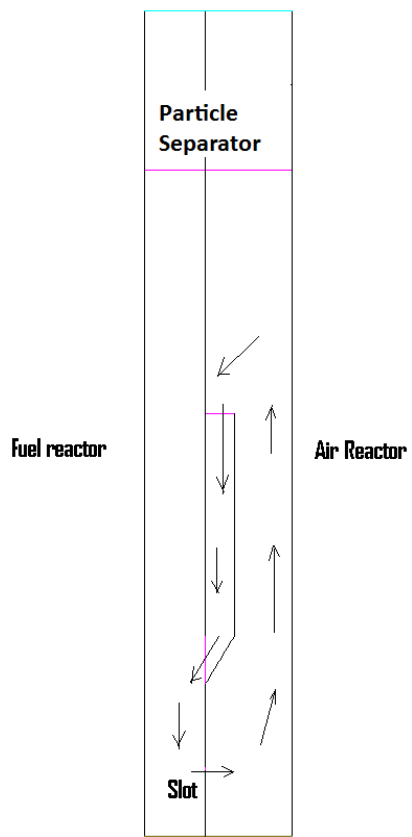

a) Actual Circulating Bed system

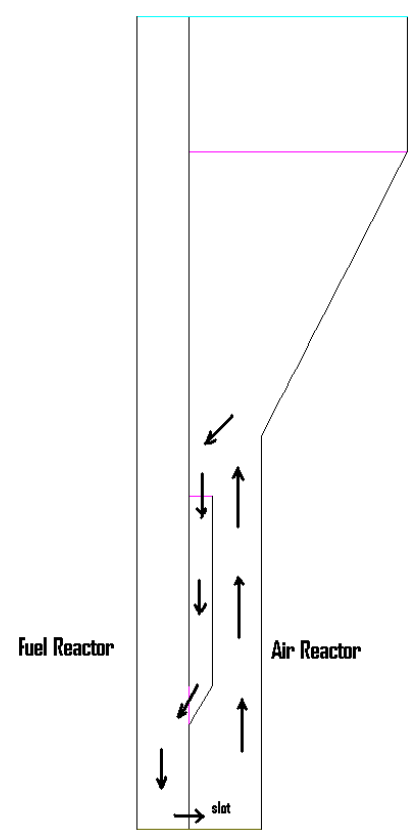

b) Computational domain for CFD

Fig. 9.1: Full CLC system studied by Kronberger et al. (2004).

Table 9.2: Numerics for simulation of Kronbeger et al. (2004) experiments.

\begin{tabular}{|l|l|}
\hline No of Cells & 25000 \\
\hline $\begin{array}{l}\text { Spatial discretization scheme for each } \\
\text { Phase conservation equation }\end{array}$ & QUICK \\
\hline $\begin{array}{l}\text { Spatial discretization scheme of other } \\
\text { equations }\end{array}$ & Second Order Upwind \\
\hline Time integration scheme & First Order Implicit \\
\hline Time step size & 0.000025 seconds \\
\hline Iterations per time step & 20 iterations \\
\hline
\end{tabular}




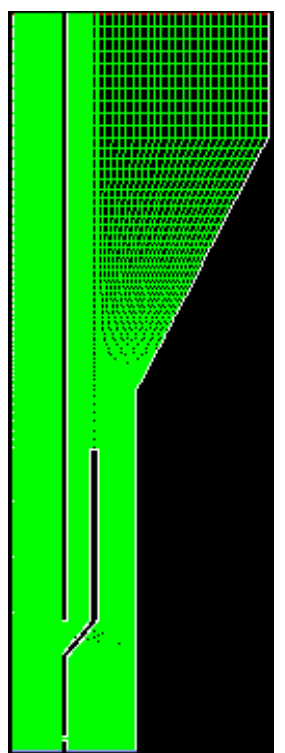

a) Mesh in whole domain

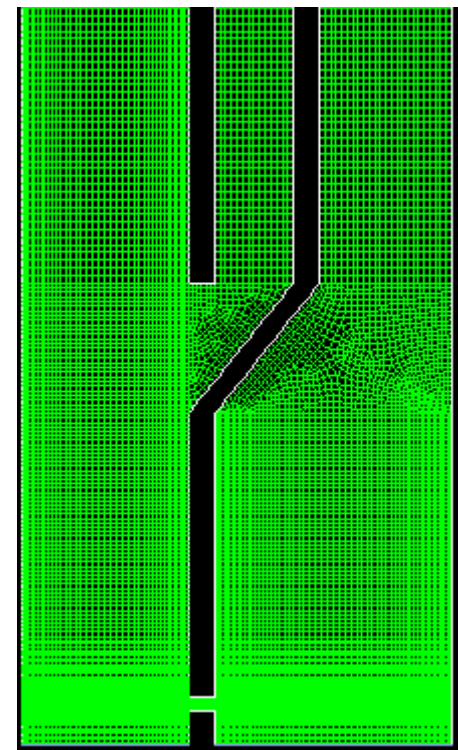

b) Mesh in downcomer

Fig. 9.2: Mesh used for simulation of Kronberger experiments (2004).

\subsubsection{Viscoplastic model for predicting frictional flow regime of granular flow:}

When the solids fraction becomes close to the dense packing limit, enduring frictional interactions between particles becomes important. Therefore, for $\alpha_{s}>0.5$, an additional frictional term is added to solid stress (see section 4.1):

$$
\bar{\tau}_{\text {friction }}=-p_{\text {friction }} \overline{\bar{I}}+\mu_{\text {friction }}\left(\nabla \vec{u}_{s}+\nabla \vec{u}_{s}^{T}\right),
$$

It is essential that the viscoplastic models used for describing the friction pressure and friction viscosity are able to predict the correct viscoplastic stresses generated and in turn predict the correct amount of energy dissipation. In the Kronberger experiments, it is reported that the frictional flow in the downcomer causes it to pack with solids and thus limit the solid circulation rates. Therefore to match experimental results the phenomenon must be accurately predicted.

Two different viscoplastic models have been tested in the present study. The first one has been developed by Schaeffer (1987) where the frictional viscosity is given by the following equation:

$\mu_{\text {friction }}=\frac{P_{\text {friction }} \sin (\phi)}{\sqrt{4 I_{2 D}}}$ 
The second model tested was a new model developed by Langroudi et al. (2010), where the frictional viscosity is given by:

$\mu_{\text {friction }}=\sqrt{2} p_{\text {friction }}\left(\frac{\sin \phi+b \cos \phi \times\left(I_{2 D}\right)^{n 1 / 2}}{\sqrt{I_{2 D}}}\right)$

Here $n 1$ is assumed to be 0.72 and $b=0.13$ (Langroudi et al., 2010).

It has been assumed that in all cases the frictional pressure is given by the Johnson and Jackson model (1987)

$$
p_{\text {friction }}=\operatorname{Fr} \frac{\left(\alpha_{s}-\alpha_{s, \min }^{*}\right)^{n}}{\left(\alpha_{s, \max }^{*}-\alpha_{s}\right)^{p}}
$$

where the values used are, $F r=5.0, n=2, p=3, \mathrm{~b}=0.13, \alpha_{s, \max }^{*}=0.63, \alpha_{s, \min }^{*}=0.5$.

Fig. 9.3 compares the computed solid circulation rates predicted by the two viscoplastic models described above. Clearly the mean solid circulation rates are considerably different in the two cases. Table 9.3 compares the computed average circulation and leakage rates to the Kronberger experiments. From these studies it is clear that for the present geometry and granular material the friction viscosity model proposed by Langroudi et al. (2010) is significantly better. The significantly lower solid circulation rates for the Langroudi et al., model is primarily because of the larger frictional viscosity predicted by it. The Langroudi et al. model assumed that the frictional viscosity has two components; one component is a direct function of the applied stress and second component is dependent on the applied strain rate. Both the FR and AR are fully fluidized and hence the volume fractions on an average are well above the frictional transition regime $\left(\alpha_{s, \min }^{*}=0.5\right)$. However in the downcomer the solid volume fraction are above the transition volume fraction and high frictional viscosities are predicted by the Langroudi model. This high frictional viscosity causes the kinetic energy of the solids to be dissipated which slows down the solid circulation rate significantly. 


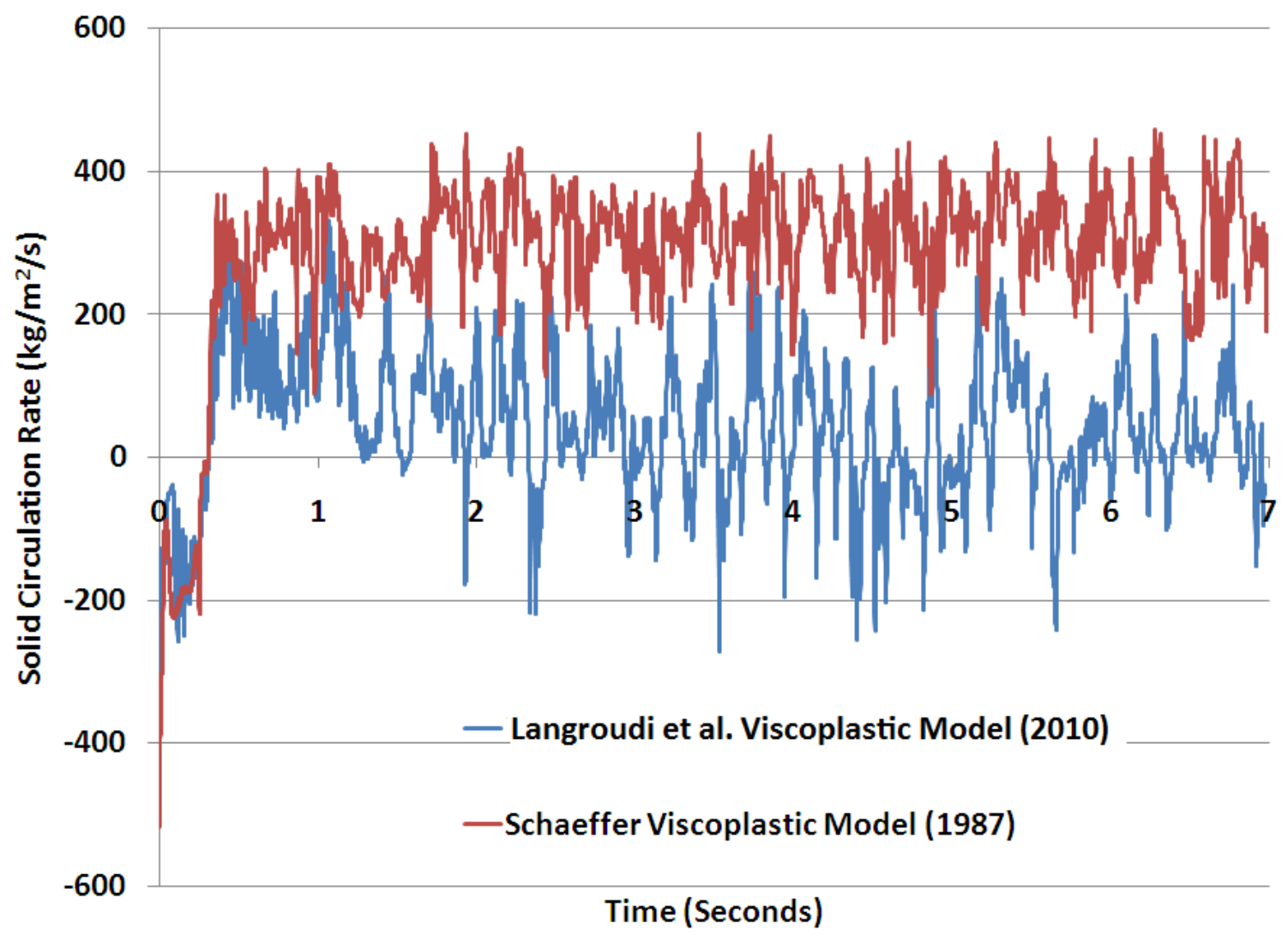

Fig. 9.3: Comparison of solid circulation rates predicted by Schaeffer model (1987) and that of Langroudi et al. (2010). Slot height for the experiment was $1.5 \mathrm{~mm}, \mathrm{U}_{\mathrm{FR}}=18 \mathrm{U}_{\mathrm{mf}}$ and $\mathrm{U}_{\mathrm{AR}}=1.8 \mathrm{U}_{\mathrm{t}}$.

Table 9. 3: Comparison of experimental and numerical simulation (time averaging for 4 seconds).

\begin{tabular}{|l|c|c|r|}
\hline & \multirow{2}{*}{ Experiment } & \multicolumn{2}{|c|}{ Simulation } \\
\cline { 4 - 4 } & & Schaeffer (1987) & Langroudi et al. (2010) \\
\hline Solid Mass Flux (kg/m $\left.{ }^{2} / \mathrm{s}\right)$ & 7.017 & 313.15 & 20.12 \\
\hline Fuel to Air Reactor Leakage (\%Flow) & $12 \%$ & $60.24 \%$ & $15.20 \%$ \\
\hline Air to Fuel Reactor Leakage (\%Flow) & $3.50 \%$ & $0 \%$ & $1.8 \%$ \\
\hline
\end{tabular}

\subsubsection{Simulations of Kronberger et al. experiments (2004):}

Fig. 9.4 shows examples of the instantaneous contour plots for solid volume fraction and solids velocity. From the volume fraction plot (Fig 9.4a) it is observed that complete filling occurs in the downcomer so that the frictional resistance of the packed column is reducing the flow of solids. The solids velocity also goes to a very low value due to this packing (Fig 9.4b). Fig. 9.5 shows a comparison 100 
between the solid circulation rates. The solid circulation rates are measured at the inlet of the downcomer where there are the least oscillations in the solid flow rates. The predicted solid circulation rate at low velocity is more than 2 times the experimental value. To some extent this can be due to 3D effects which are likely to be more pronounced at low velocity. Fig. 9.6 shows the gas leakage into the air reactor. The comparison with experiment is reasonable. Fig.9.7 shows the gas leakage into the fuel reactor; due to the low percentage of leakage these results off by a factor of about 2 . From these results it is clear that the experimental trends are captured very well with the present friction viscosity model. Given the extremely complex frictional dissipative phenomena that occur in the downcomer it is not surprising that some of the quantitative values are differ by $50 \%$ when compared with experiments. Also, Kronberger et al. indicate that the solid circulation rates differ by an order of magnitude when slightly different solid particles are used (e.g. similar size glass beads). This clearly highlights the complex nature of granular frictional flow and difficulties in modeling it accurately.

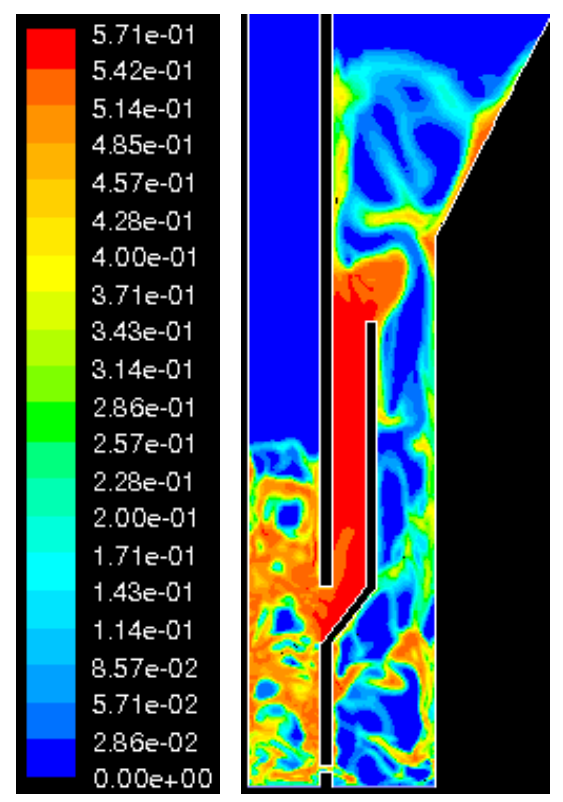

a) Volume fraction plots
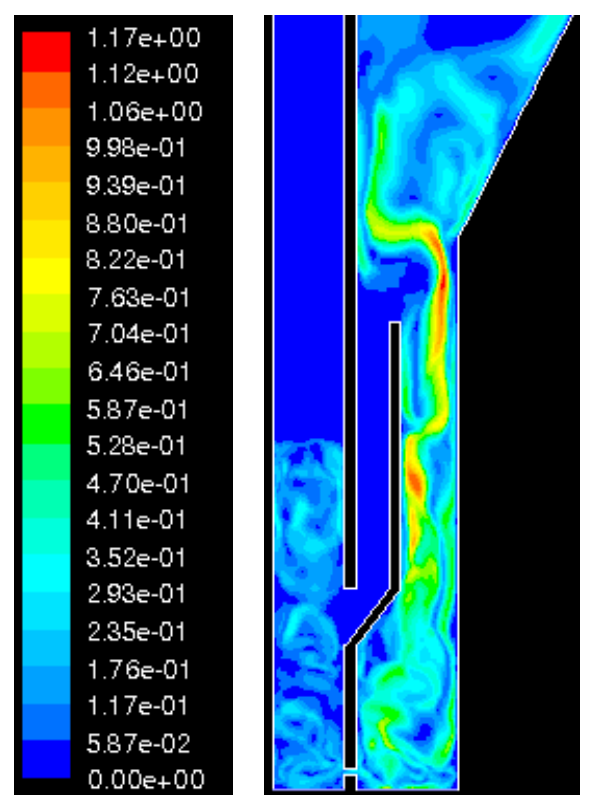

b) Solid particle velocity $(\mathrm{m} / \mathrm{s})$

Fig. 9.4: Instantaneous contour plots at 6 seconds $\left(U_{F R}=18 U_{m f}\right.$ and $\left.U_{A R}=1.8 U_{t}\right)$. 


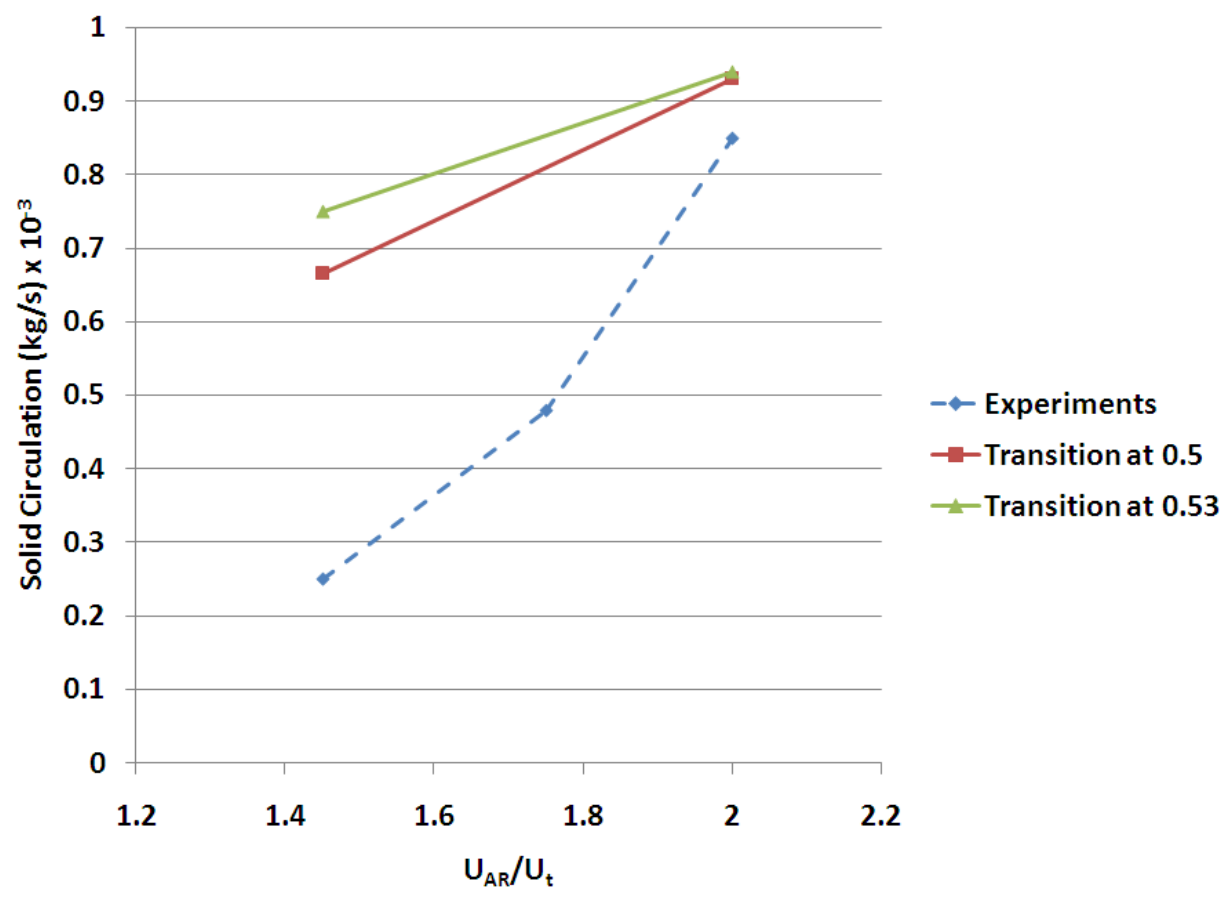

Fig. 9.5: Comparison of solid circulation rates with experiments. Two values of transition volume fraction $\alpha_{s, \min }^{*}$ have been used $(0.5$ and 0.53$)$ to check the sensitivity of this parameter $\left(U_{\mathrm{FR}}=18 \mathrm{U}_{\mathrm{mf}}\right)$.

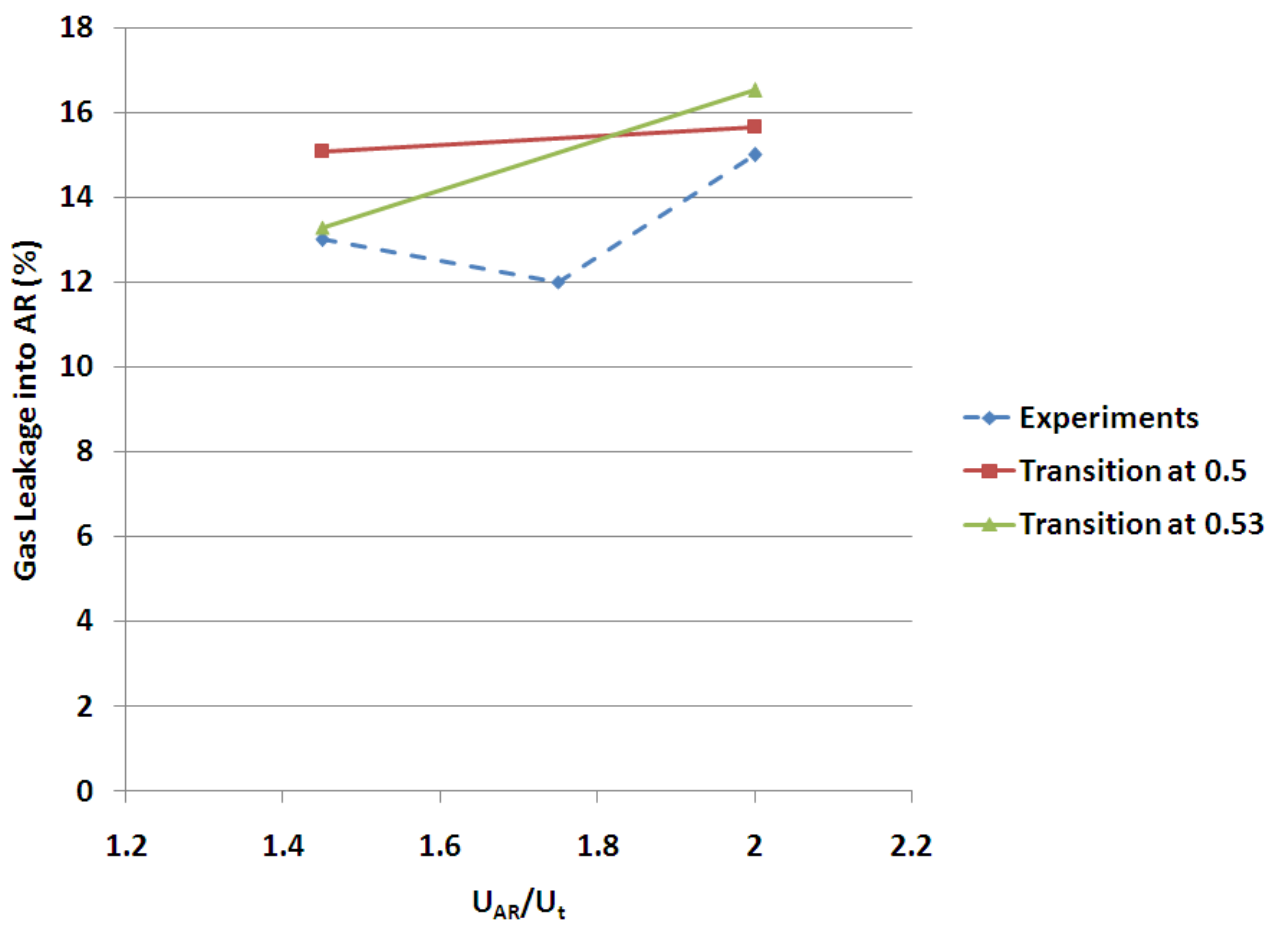

Fig. 9.6: Gas leakage into Air Reactor. Two values of transition volume fraction $\alpha^{*}{ }_{s, \min }$ have been used ( 0.5 and 0.53$)$ to check the sensitivity of this parameter $\left(U_{\mathrm{FR}}=18 U_{\mathrm{mf}}\right)$. 


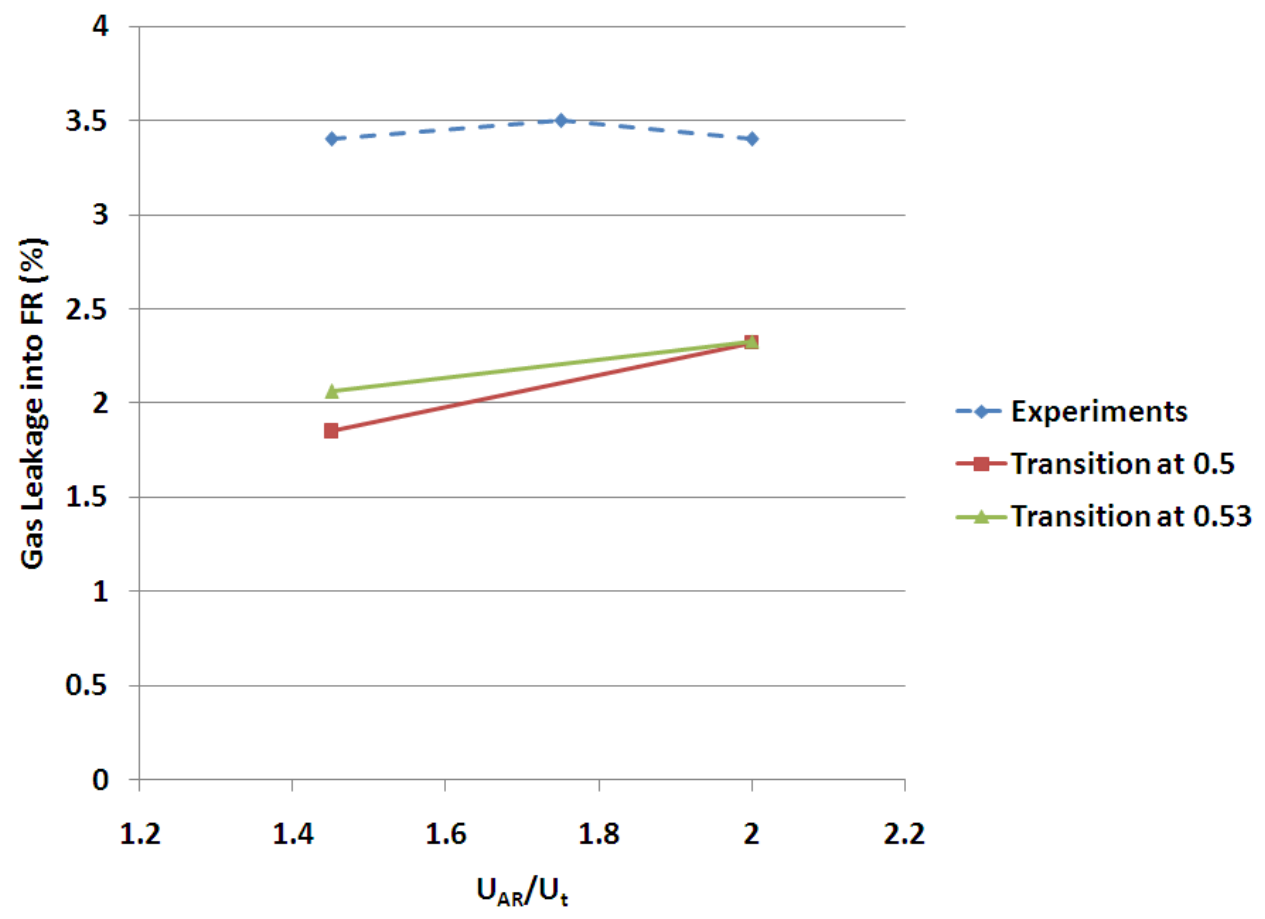

Fig. 9.7: Gas leakage into Fuel Reactor. Two values of transition volume fraction $\alpha^{*}$ s,min have been used $(0.5$ and 0.53$)$ to check the sensitivity of this parameter $\left(U_{F R}=18 U_{m f}\right)$.

\subsection{Details of Abad et al. (2006) experimental study}

The experiments of Abad et al. (2006) are for a hot flow reactor which utilized a metal oxide carrier consisting of manganese oxide particles supported on zirconium oxide. The gaseous fuel used is methane, and is used for fluidizing the fuel reactor. The air reactor fluidizing velocity is higher than the terminal velocity of the particles and therefore carries the particles upwards. As in the Kronberger cold flow geometry, at the top section there is a sudden expansion of flow due to the presence of a particle separator, and this causes the particles to fall back down. In the experiments the particle separator consists of an expansion perpendicular to plane of paper. Similar to the Kronberger experiments the geometry has been modified such that the area expansion is in the 2D plane being simulated (Fig 9.1). Since this expansion occurs in the downstream of the circulating bed it is unlikely to affect the solution significantly. Table 9.4 provides the details of geometry and fluidization conditions. 
Table 9.4: System properties for experiments of Abad et al. (2006).

\begin{tabular}{|l|l|}
\hline Width of fuel reactor $(\mathrm{mm})$ & 25 \\
\hline Width of air reactor $(\mathrm{mm})$ & 40 \\
\hline Width of downcommer $(\mathrm{mm})$ & 12 \\
\hline Fluidizing gas composition in fuel reactor & $100 \% \mathrm{CH}_{4}$ \\
\hline Fluidizing gas composition in air reactor & $100 \%$ Air \\
\hline Average diameter of particles $(\mathrm{microns})$ & 150 \\
\hline Average density of particles $\left(\mathrm{kg} / \mathrm{m}^{3}\right)$ & 2260 \\
\hline Range of fuel reactor flow rates $\left(\mathrm{m}^{3} / \mathrm{s}\right)$ & $2.5 \times 10^{-6}$ to $7.5 \times 10^{-6}$ \\
\hline Range of air reactor flow rates $\left(\mathrm{m}^{3} / \mathrm{s}\right)$ & $66.7 \times 10^{-6}$ to $91.7 \times 10^{-6}$ \\
\hline Mass of metal oxide particle bed $(\mathrm{g})$ & $300 \mathrm{~g}$ \\
\hline
\end{tabular}

\subsubsection{Details of Numerics for Abad et al. (2006) experiments:}

Three different grid sizes (coarse, medium and fine mesh) were used in the present study to investigate grid independence. The spatial discretization scheme for volume fraction equations was the quadratic upwind interpolation scheme (QUICK). A second order upwind discretization was used for all other equations. The CFD domain for the geometry of the FR is shown in Fig. 9.8. The grid used in the simulation was a quadrilateral cell grid (Fig. 9.8). The temporal discretization scheme in all cases was first order implicit. The cell count and time step used are presented in Table 9.5. A large computational time was required for the fine mesh due to the small time steps used. The small time steps are essential for numerical stability of the solution. Due to this reason the results presented are primarily using the medium size mesh unless stated otherwise. The Table 9.5 also shows the number of cells used to resolve the width of the downcomer and slot, which are responsible for limiting the transfer of solid mass between AR and FR. It is likely that the flow in the downcomer and slot have to be well resolved to obtain accurate solid circulation rates. 

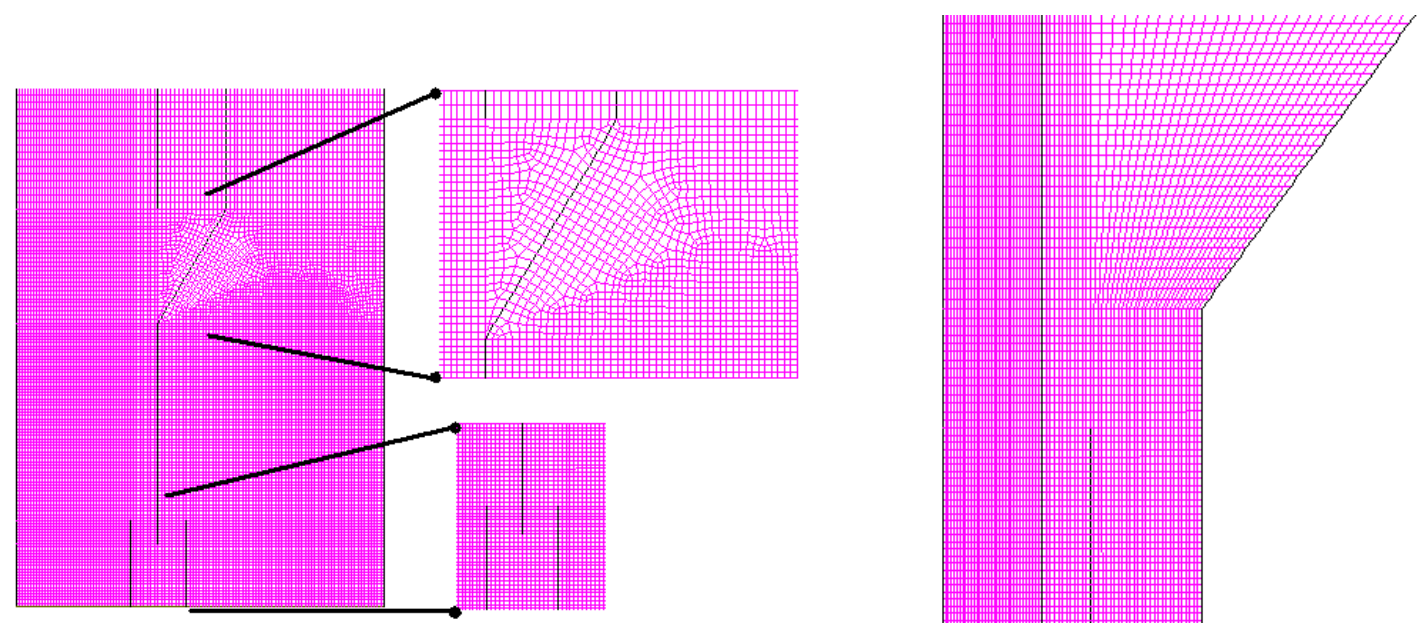

a) Mesh at the bottom of CFD domain

b) Mesh used at top of the CFD domain

Fig. 9.8: Fine mesh used in the numerical study.

Table 9.5: Numerics for simulation of Abad experiments (2004).

\begin{tabular}{|l|c|c|c|}
\hline & $\begin{array}{l}\text { Coarse } \\
\text { Mesh }\end{array}$ & $\begin{array}{l}\text { Medium } \\
\text { Mesh }\end{array}$ & $\begin{array}{l}\text { Fine } \\
\text { Mesh }\end{array}$ \\
\hline Cell count & 8822 & 17562 & 33745 \\
\hline Number of cells to resolve slot width & 8 & 12 & 20 \\
\hline Number of cells to resolve downcomer width & 6 & 10 & 15 \\
\hline Time step (sec) & $2 \times 10^{-4}$ & $1 \times 10^{-4}$ & $2.5 \times 10^{-5}$ \\
\hline
\end{tabular}

\subsubsection{Chemical Reactions and Kinetics used:}

The carrier used in the experiments of Abad et al. (2006) was manganese oxide supported on zirconium oxide. Therefore the following reaction mechanisms were used:

Reduction Reaction:

$4 \mathrm{Mn}_{3} \mathrm{O}_{4}+\mathrm{CH}_{4} \rightarrow 12 \mathrm{MnO}+\mathrm{CO}_{2}+2 \mathrm{H}_{2} \mathrm{O}$

Oxidation Reaction:

$12 \mathrm{MnO}+2 \mathrm{O}_{2} \rightarrow 4 \mathrm{Mn}_{3} \mathrm{O}_{4}$

$\mathrm{CH}_{4}+2 \mathrm{O}_{2} \rightarrow \mathrm{CO}_{2}+2 \mathrm{H}_{2} \mathrm{O}$

Son and Kim (2006) suggest that the uniform reaction model can best represent the heterogeneous chemical reactions of metal oxides in a CLC system. The uniform reaction model is given as: 


$$
\frac{d X}{d t}=k(1-X)
$$

where $\mathrm{X}$ is the conversion and is given as:

$$
X=\frac{m-m_{r e d}}{m_{o x}-m_{r e d}},
$$

where ' $m_{o x}$ ' is the oxidized (ox) form i.e. the sum of mass of $\mathrm{Mn}_{3} \mathrm{O}_{4}$ and ' $\mathrm{m}_{\text {red }}$ ' is the reduced (red) form MnO. Similarly Mattisson et al. (2005) suggest that a changing grain model based on rates controlled by slow chemical reactions is suitable. The reaction rate suggested by them is given as

$$
\frac{d X}{d t}=k(1-X)^{2 / 3}
$$

In the present case the uniform reaction model as suggested by Son and Kim, 2006 is used. The coefficient ' $\mathrm{k}$ ' itself is given by the Arrhenius equation given below:

$$
k=k_{o} e^{-E / R T}
$$

Adanez et al. (2004) have carried out studies on manganese oxide particles with the same type and percentage of support material as that in present case. They used thermo-gravimetric analyzer to determine the heterogeneous reaction rates. Based on their studies the following constants for the reduction reaction have been estimated (see Fig. 4 Adanez et al., 2004):

$k_{o}=2943.5151 / \mathrm{sec}$

$E=104628.4 \mathrm{~J} / \mathrm{K} / \mathrm{mol}$

For the oxidation reaction, Adanez et al. (2004) have shown that the reaction rate is almost constant within the temperature range of the present experiments. The coefficient ' $k$ ' for the oxidation reaction is estimated to be 0.1 .

Differentiating Equation 9.8 yields: 
$\frac{d X}{d t}=\frac{1}{m_{o x}-m_{r e d}} \frac{d m}{d t}=\frac{1}{R_{o} m_{o x}} \frac{d m}{d t}$

where $R_{o}=\frac{m_{o x}-m_{r e d}}{m_{o x}}$

All the loss in mass of metal oxide is due to of loss of oxygen. Therefore

$d m=d m_{O 2}=M W_{O 2} \times d n_{O 2}$.

Further it can be shown that the rate of consumption of methane $\left(\mathrm{kg} /\left(\mathrm{m}^{3} \mathrm{~s}\right)\right)$ is given by:

$\dot{m}_{\mathrm{CH}_{4}}=\frac{k R_{o}}{2 M W_{\mathrm{O}_{2}}}(1-X) \rho_{\text {avg }} \varepsilon_{s}\left(Y_{\mathrm{Mn}_{3} \mathrm{O}_{4}}+Y_{\mathrm{MnO}} \times \frac{v_{\mathrm{Mn}_{3} \mathrm{O}_{4}} M W_{\mathrm{Mn}_{3} \mathrm{O}_{4}}}{v_{M n O} M W_{M n O}}\right) \frac{Y_{\mathrm{CH}_{4}}}{Y_{\mathrm{CH}_{4}-T G A}} M W_{\mathrm{CH}_{4}}$

$Y_{C H 4_{-} T G A}$ is the methane mass fraction in TGA experiments during the reducing phase of the experiment. These rates have been scaled linearly for other concentration values. The concentration of $\mathrm{CH}_{4}$ in TGA was $70 \%\left(70 \% \mathrm{CH}_{4}\right.$ and $\left.30 \% \mathrm{H}_{2} \mathrm{O}\right)$ or $Y_{\mathrm{CH}_{4-} T G A}=0.675$.

Similarly the reaction rate for the oxidation reaction $\left(\mathrm{kg} /\left(\mathrm{m}^{3} \mathrm{~s}\right)\right)$ can be shown to be

$\dot{m}_{O_{2}}=\frac{k R_{o}}{M W_{O_{2}}}(X) \rho_{\text {avg }} \varepsilon_{s}\left(Y_{M n_{3} O_{4}}+Y_{M n O} \times \frac{v_{M n_{3} O_{4}} M W_{M n_{3} O_{4}}}{v_{M n O} M W_{M n O}}\right) \frac{Y_{O_{2}}}{Y_{O_{2}-T G A}} M W_{O_{2}}$

$Y_{O_{2}-T G A}$ is the oxygen mass fraction in TGA experiments during the oxidizing phase of the experiment. Since the experiments of Adanez et al. were carried out using air, $Y_{O_{2}{ }_{-} T G A}=0.23$.

DeSouza-Santos (2004), provide global reaction rates for oxidation of methane in systems utilizing coal and biomass as fuel. They provide the reaction rate as

$\dot{m}_{C_{4}}=k_{C H_{4}} e^{(-E / R T} T^{-1}\left(C_{C_{4}}\right)^{m}\left(C_{O_{2}}\right)^{n}$

Where $k_{\mathrm{CH}_{4}}=3.552 \times 10^{14}\left(\mathrm{kmol} / \mathrm{m}^{3}\right)^{1-m-n} / \mathrm{s} ; E / R=15700 \mathrm{~K} ; \mathrm{m}=1.0$ and $\mathrm{n}=1.0$. 


\subsubsection{Results from simulation of Abad et al. (2006) experiments:}

Fig. 9.9a shows the volume fraction of the gas phase at one time instant. The formation and rise of bubbles can be clearly seen in the fuel reactor. The downcomer region of the reactor was observed to be completely packed. This packing is attributed to the energy dissipated due to dense frictional flow in the downcomer that was also observed in the cold flow experimental studies of the same system by Kronberger et al. (2004). The packing in the downcomer also limits the solid circulation rates. Fig. 9.9 also shows examples of the computed mole fraction of various gases. Fig. 9.9b shows the rapid consumption of methane as it enters the fuel reactor. Also, a higher concentration of methane was observed in the bubble regions indicating that some amount of fuel bypasses the bed through the bubbles. Fig. 9.9c shows the mole concentration of $\mathrm{CO}_{2}$ at operating temperature of $1123 \mathrm{~K}$. The $\mathrm{CO}_{2}$ concentration is low at the inlet region but increases with bed height as the methane reacts with the metal oxide. Some leakage of $\mathrm{CO}_{2}$ into the air reactor can be observed.

It is desirable that the CLC reactor consumes all the supplied fuel and reaches a high combustion efficiency. This can be achieved by having more solids inventory in the system, but this will also result in higher power consumption to fluidize and circulate the solid particles. Therefore there is a need to achieve an optimum level of solid inventory. The CFD model used should be able to accurately capture the methane consumption at a given solid inventory. Fig. 9.10 shows the comparison of the variation of outlet $\mathrm{CH}_{4}$ mole fraction with change in the fuel reactor gas flow rate. At lower flow rates the time required for the fuel to flow across the FR bed height is larger and hence it has more time to react with the metal oxides. The outlet concentration of fuel is therefore lower at lower flow rates. Also the bubble size and frequency are lower at lower flow rates and hence the amount of fuel escaping through the bubbles is also lower. The trends in outlet $\mathrm{CH}_{4}$ concentration are captured reasonably well for the entire range of flow rates tested. The differences in the outlet methane concentrations are attributed to three main reasons:

1) The method of preparation for the metal oxide used by Abad et al. (2006) was different than that of Adanez et al. (2004). This could result in some differences in porosity and available particle surface area of the metal oxide which can cause differences in the reaction rates.

2) The chemistry model used is a curve fit to available experimental data from a thermogravimetric analyzer (TGA). Also the TGA experiments were carried out at a single concentration of methane by flooding the reaction compartment with an excess amount of 
gases. In an actual experimental system the methane concentration will vary from $100 \%$ to close to $0 \%$. A linear scaling in reaction rates has been assumed with respect to concentration of methane. This may not necessarily be accurate.

3) Errors in fluid mechanics such as error in the bubble size or frequency can cause the outlet concentration to vary. As discussed above, the bubbles allow the leakage of gases through them. Detailed simulations of Hulme et al. (2005) and Chandrasekaran et al. (2005) have shown that the fluid mechanics can be reasonably predicted by using mathematical models that are similar to that used in the present study.

Fig. 9.11 shows the variation of outlet $\mathrm{CH}_{4}$ concentration with change in operating temperature as well as changes in AR air flow rates. When the operating temperature was varied the FR flow rate and AR flow rates were held constant at $2.5 \times 10^{-6}$ and $7.5 \times 10^{-6} \mathrm{~m}^{3} / \mathrm{s}$ respectively. When the AR flow rate was varied then the reactor temperature was held constant at $1123 \mathrm{~K}$ and the FR flow rate was $5.8 \times 10^{-6}$ $\mathrm{m}^{3} / \mathrm{s}$. Once again the CFD model predicts trends in a reasonable manner. At low operating temperature the reaction rates are lower resulting in higher fuel concentrations at the exit. At a higher temperature of $1223 \mathrm{~K}$ the outlet fuel fraction is less than $1 \%$. The outlet fuel fraction does not vary significantly with change in air flow rates. This is primarily because the solids circulation rates as well the solid mass in the fuel reactor do not change significantly with changes in air flow rates. The reasons for this are discussed below.

Fig. 9.12 shows the variation in solid circulation rates with changes in the fuel flow rates. Predicting solid circulation rates accurately is important as it determines the amount of oxygen supplied for burning the fuel as well as the energy transfer between the exothermic air reactor and the endothermic fuel reactor. The solids circulation is the primary means of energy transfer between AR and FR. The solids are observed to pack in the downcomer (Fig. 9.9a). The frictional energy dissipation in the downcomer of the CLC system is the primary controlling mechanism of the solids circulation rate. This has been verified by running the simulations without any frictional stresses (i.e. excluding Equation 9.1). It was found that without frictional stresses, the predicted circulation rates increased by a factor of 100 in comparison with the experimental results. The circulation rate is computed at the downcomer inlet which has the least oscillations in mass flux. From Fig. 9.12 it is observed that the coarse mesh predicts a significantly larger flow rate in comparison with the fine mesh. The differences in coarse and 
fine mesh results can be attributed to numerical diffusion and other discretization errors. A coarse mesh has higher numerical diffusion of solid phase resulting in lower solids volume fraction in comparison with the finer mesh. A lower solid volume fraction will result in lower frictional stresses (as well as frictional energy dissipation). The lower frictional energy dissipation causes the solid circulation rates to be higher. From Fig. 9.12 it can be observed that grid refinement results in more accurate prediction of solid circulation rates. Also both medium and fine mesh predict a slight increase in the solid circulation rates with increase in fuel flow. This is similar to the trend observed in the experiments.

Part of the reason for differences in simulated and experimental solid flow rates can also be attributed to the following:

1) In the present simulations, models based on plasticity theory have been used to model the frictional stresses of solids. Since the frictional flow is extremely complex, the plasticity theory is only an approximate representation of the flow physics.

2) The experimental solid circulation rates were calculated indirectly from transient increase in oxygen concentration after stopping the combustion test. Due to the indirect method used for measuring solid circulation rates, it is likely to have some error.

The simulations also capture the slight increase in the solid circulation rates with increase in air flow (Fig. 9.13). The differences in simulated and experimental values are attributed to reasons discussed above.

Another important parameter is the distribution of solid mass in the fuel and air reactor. The amount of solid mass in the fuel reactor will directly affect the amount of methane burned by the reactor and hence it has to be predicted accurately. Table 9.6 shows the experimental and predicted mass in the air and fuel reactors. The mass in the AR and FR is computed using two different methods:

i. $\quad$ Pressure drop across the reactor (Mass $=\Delta P \times$ Area $/ g)$

ii. Direct integration/summation of solid mass in all cells.

The predicted variation in mass of solid inventory in the fuel and air reactor with respect to changes in the fluidizing velocity is less than $2 \%$. This is similar to that observed in experiments of Abad et al. (2006) where the mass of solid inventory remained more or less constant in different cases that were tested. The difference in computed mass in the fuel reactor is within $11 \%$ of the experimentally 110 
observed values (105 grams versus 94 grams, Table 9.6). The experimentally observed mass in the air reactor was 110 grams and the computed mass using pressure difference was around 117 grams resulting in an error of around 7\% (Table 9.6). The computed air reactor mass found using pressure difference is significantly different from that obtained by direct integration of solid mass in the reactors (117 grams versus 156 grams). This difference is attributed to the fact that the mass of solid particles found by measuring the pressure difference is unlikely to account for the mass of solid particles deposited on the inclined walls of the particle separator, as they do not contribute to the pressure difference. The main reason for the differences in the simulations and experimental results is attributed to modeling assumptions such as drag law, stress-strain relationships, packing limit etc. In the simulations the packing limit was assumed to be 0.63 , however the exact value is unknown. A higher/lower packing limit in the downcomer will change the solid mass distribution in the reactors.

Table 9.6: Mass of metal oxides (grams).

\begin{tabular}{|l|r|r|r|}
\hline & $\begin{array}{c}\text { Experimental } \\
\text { (From } \\
\text { Pressure } \\
\text { drop) }\end{array}$ & $\begin{array}{c}\text { CFD Simulations } \\
\text { (From Pressure } \\
\text { drop) }\end{array}$ & $\begin{array}{c}\text { CFD Simulations } \\
\text { (From Direct Mass } \\
\text { Integration) }\end{array}$ \\
\hline Solid Mass in Fuel reactor (grams) & 105 & 94 & 97 \\
\hline Solid Mass in Air Reactor (grams) & 110 & 117 & 156 \\
\hline
\end{tabular}

The magnitude of gas leakage between the $A R$ and $F R$ is another important parameter to be considered in the design of a CLC system. Leakage and dilution are undesirable in a chemical looping reactor as they cause $\mathrm{CO}_{2}$ to be released to the atmosphere and also dilute the concentrated $\mathrm{CO}_{2}$ stream at the exit of the FR. Therefore accurate predictions of these quantities by the computational model are desirable.

Abad et al. (2006) define leakage as the percentage of gas flow from FR to AR and dilution as the percentage of gas flow from AR to FR. Leakage and dilution are related to the incoming fuel flow and are defined as:

$$
L=\frac{F_{L}}{F_{i n, F R}} \quad \text { and } \quad D=\frac{F_{D}}{F_{i n, F R}}
$$

Here $F_{L}$ is the flow of gas leaking from the fuel to the air reactor, $F_{i n, F R}$ is the flow of gas entering the fuel reactor and $F_{D}$ is the flow of gas leaking from AR to FR. In the simulations the leakage 111 
is measured by the $\mathrm{CO}_{2}$ concentrations at the exit of $\mathrm{AR}$ and the dilution was measured by the $\mathrm{N}_{2}$ concentration at the exit of FR which is similar to that in the experimental study (Johansson et al., 2006).

The leakage and dilution of gas occurs through both the slot and the downcomer. Fig. 9.14 shows the computed gas mass flow rates for a time interval of 1.5 seconds at the downcomer inlet (entrance/inlet is on the AR side) and the slot connecting FR and AR. It is observed that the gas flow through both downcomer and slot is of similar order of magnitude. In the downcomer the flow of gases is primarily from AR to FR. This is despite the presence of a large adverse pressure gradient trying to push the gases from FR to AR. The packing of the downcomer provides high resistance to the gas flow and hence tends to prevent the flow of gases from the fuel reactor to the air reactor. Also the flow of solid particles causes a significant amount of gases to be dragged with the solids from the AR into FR via the downcomer. In the slot, the gas flow is primarily from FR to AR. The gas pressure is on average higher in the fuel reactor side and lower on the air reactor. The gas flow also drags the solid particles along with it thus helping with the transport of particles between reactors. The gas pressure oscillations occur due to formation and rise of bubbles; this can cause a temporary reverse flow of gases from the AR to FR leading to dilution.

Fig. 9.15 shows the computed leakage and dilution versus the change in the fuel flow rates. The predicted leakage and dilution are between 3 to 10\%. Abad et al. (2006) report the experimental leakage and dilution in the range of 3 to $30 \%$. In general the amount of gas exchange between the reactors increases with increase in fuel flow (i.e. $F_{L}$ and $F_{D}$ increase). However the leakage (L) decreases primarily because of the increase in $F_{i n, F R}$. Also the increase in fuel flow rates increases gas pressure on the FR side causing a decrease in the flow of gases from AR to FR. This results in decrease in dilution with change in fuel flow rates. Fig. 9.16 shows the computed variation in leakage and dilution versus the increase in air flow. In general both leakage and dilution increase with increase in air flow. The increase in leakage is primarily because of higher gas pressures in the FR side causing higher gas flow as well as solids flow through the slot. The increase in dilution is primarily because of the increased amount of gas being dragged from the AR to FR through the downcomer due to higher solids flow rate. The trends observed in the simulations are similar to that observed by Johansson et al. (2006) in experiments on the same system but with different type of solid particles and different amount of solid inventory. 
Fig. 9.17 shows the variation in carbon capture efficiency with change in the fuel flow rate and air flow rate, where the carbon capture efficiency is defined as:

$\eta_{C C}=\frac{\mathrm{CO}_{2} \text { Flow Rate at Exit of } \mathrm{AR}}{\mathrm{CO}_{2} \text { Flow Rate at Exit of } \mathrm{AR}+\mathrm{CO}_{2} \text { Flow Rate at Exit of FR }}$

High carbon capture efficiency in excess of $90 \%$ is predicted by the simulations. There is a slight increase in the carbon capture efficiency with increase in fuel flow rates. This is primarily because the amount of $\mathrm{CH}_{4}$ and $\mathrm{CO}_{2}$ leaking into the $\mathrm{AR}$ increases marginally while the total $\mathrm{CO}_{2}$ outflow in the $\mathrm{FR}$ (as well as inlet $\mathrm{CH}_{4}$ flow rates) increases by more than a factor of 3. Also the carbon capture efficiency decreases with increase in air reactor flow rates (Fig. 9.17). This is primarily because the increase in the air reactor flow rates causes a higher gas pressure on the fuel reactor side which results in higher leakage of gases from FR to AR.
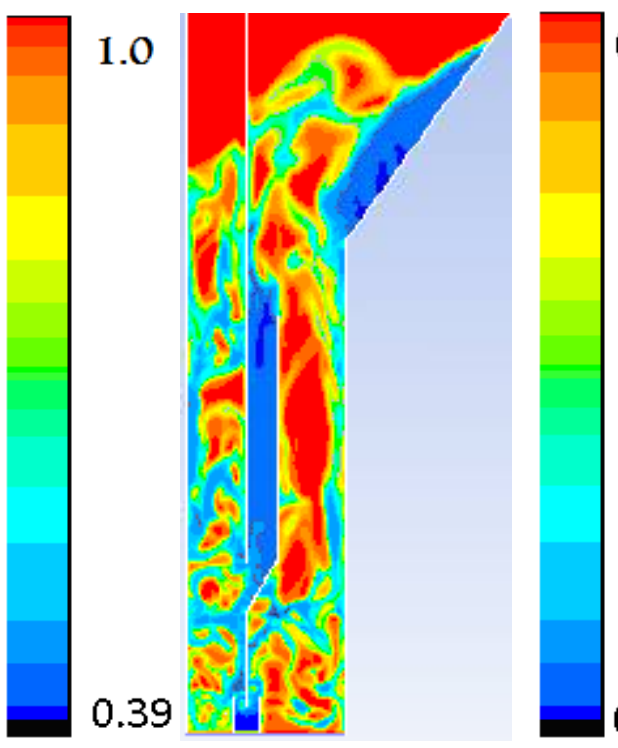

a) Gas volume frac.

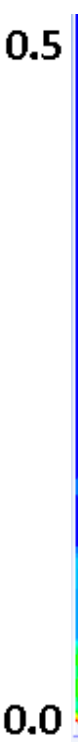

0.0

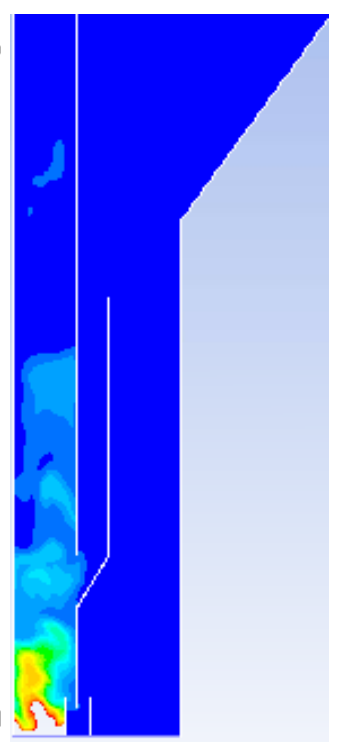

b) $\mathrm{CH}_{4}$
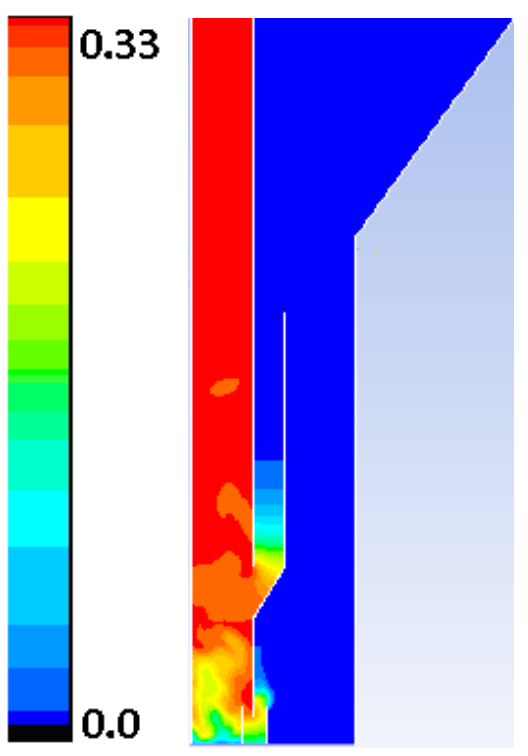

c) $\mathrm{CO}_{2}$

Fig. 9.9: Gas volume fraction and species mole fraction contour of various gases (Operating temperature of $1123 \mathrm{~K})$. FR flow rate of $7.5 \times 10^{-6} \mathrm{~m}^{3} / \mathrm{s}$ and AR flow rate of $83 \times 10^{-6} \mathrm{~m}^{3} / \mathrm{s}$. 


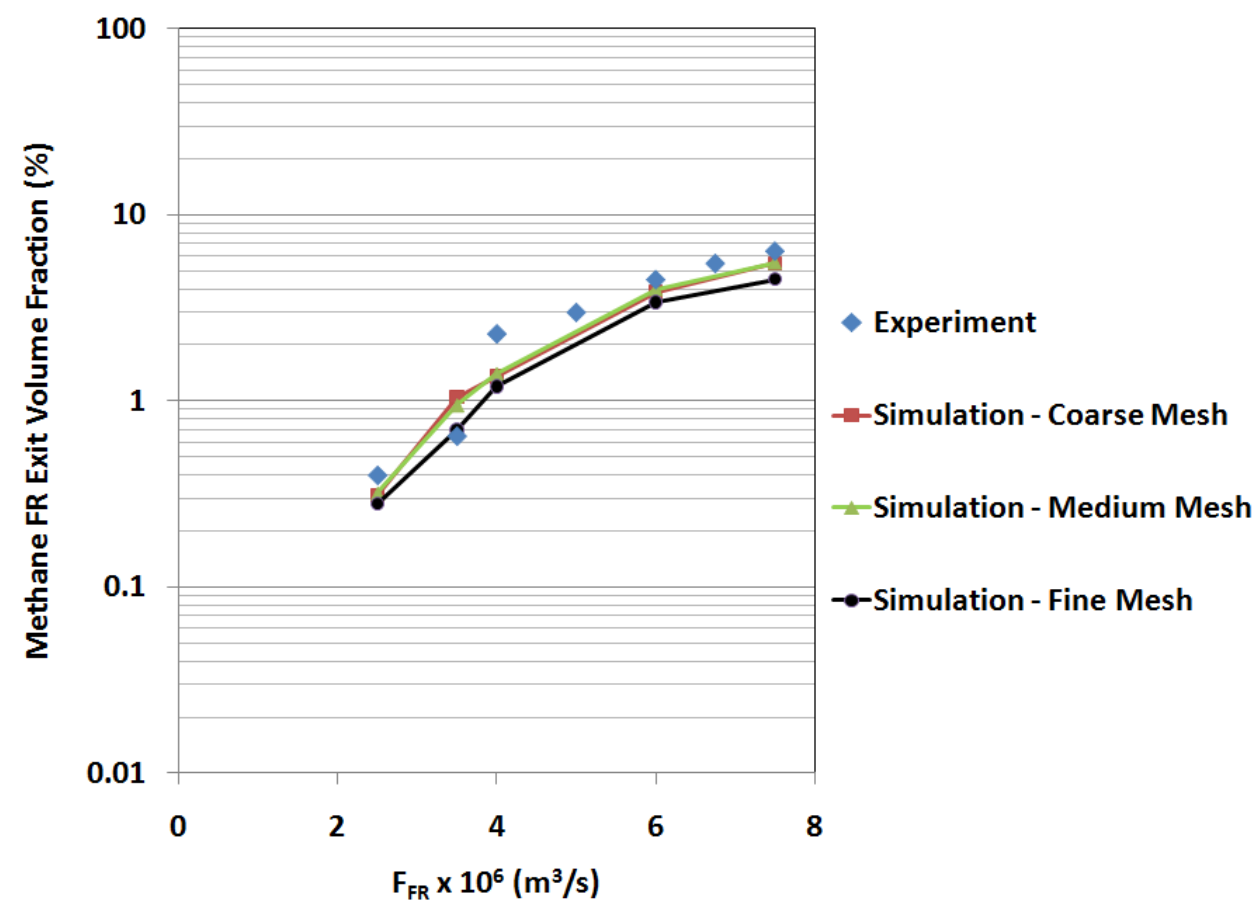

Fig. 9.10: Variation in outlet $\mathrm{CH}_{4}$ fraction $\left(\mathrm{X}_{\mathrm{CH} 4} /\left(\mathrm{X}_{\mathrm{CH} 4}+\mathrm{X}_{\mathrm{CO}}+\mathrm{X}_{\mathrm{CO} 2}\right)\right)$, with change in FR flow rates (FR flow rates varies from $2.5 \times 10^{-6}$ to $7.5 \times 10^{-6} \mathrm{~m}^{3} / \mathrm{s}$ and $A R$ flow rates is constant at $83 \times 10^{-6} \mathrm{~m}^{3} / \mathrm{s}$ ).

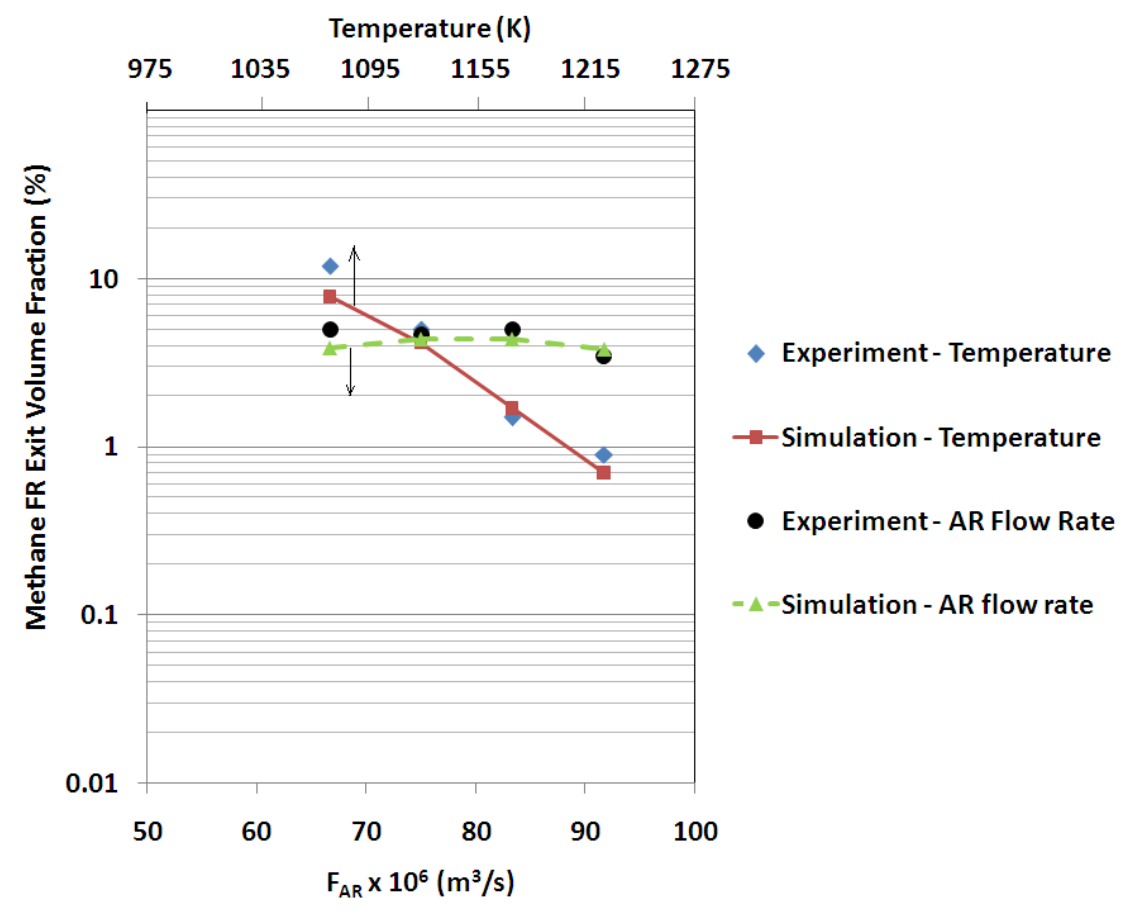

Fig. 9.11: Variation in outlet $\mathrm{CH}_{4}$ fraction $\left(\mathrm{X}_{\mathrm{CH} 4} /\left(\mathrm{X}_{\mathrm{CH} 4}+\mathrm{X}_{\mathrm{CO}}+\mathrm{X}_{\mathrm{CO} 2}\right)\right)$, with change in operating temperature of oven (Temperature changes from 1073K to 1223K) and change in AR flow rates (AR flow rates varies from $66.7 \times 10^{-6}$ to $91.7 \times 10^{-6} \mathrm{~m}^{3} / \mathrm{s}$ ). 


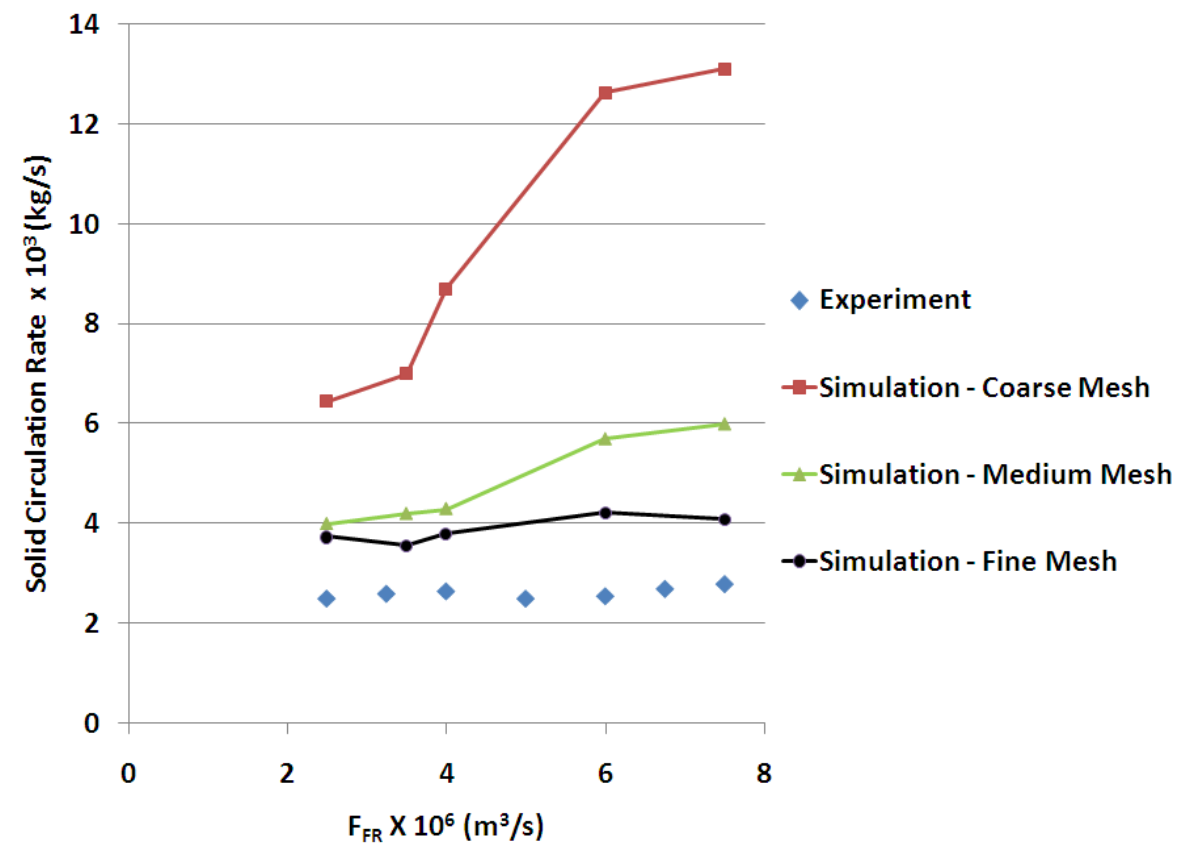

Fig. 9.12: Variation in solid circulation rate $\left(\mathrm{kg} / \mathrm{s} \times 10^{3}\right)$ with change in fuel reactor flow rates (FR flow rates varies from $2.5 \times 10^{-6}$ to $7.5 \times 10^{-6} \mathrm{~m}^{3} / \mathrm{s}$ ). AR $=83 \times 10^{-6} \mathrm{~m}^{3} / \mathrm{s}$.

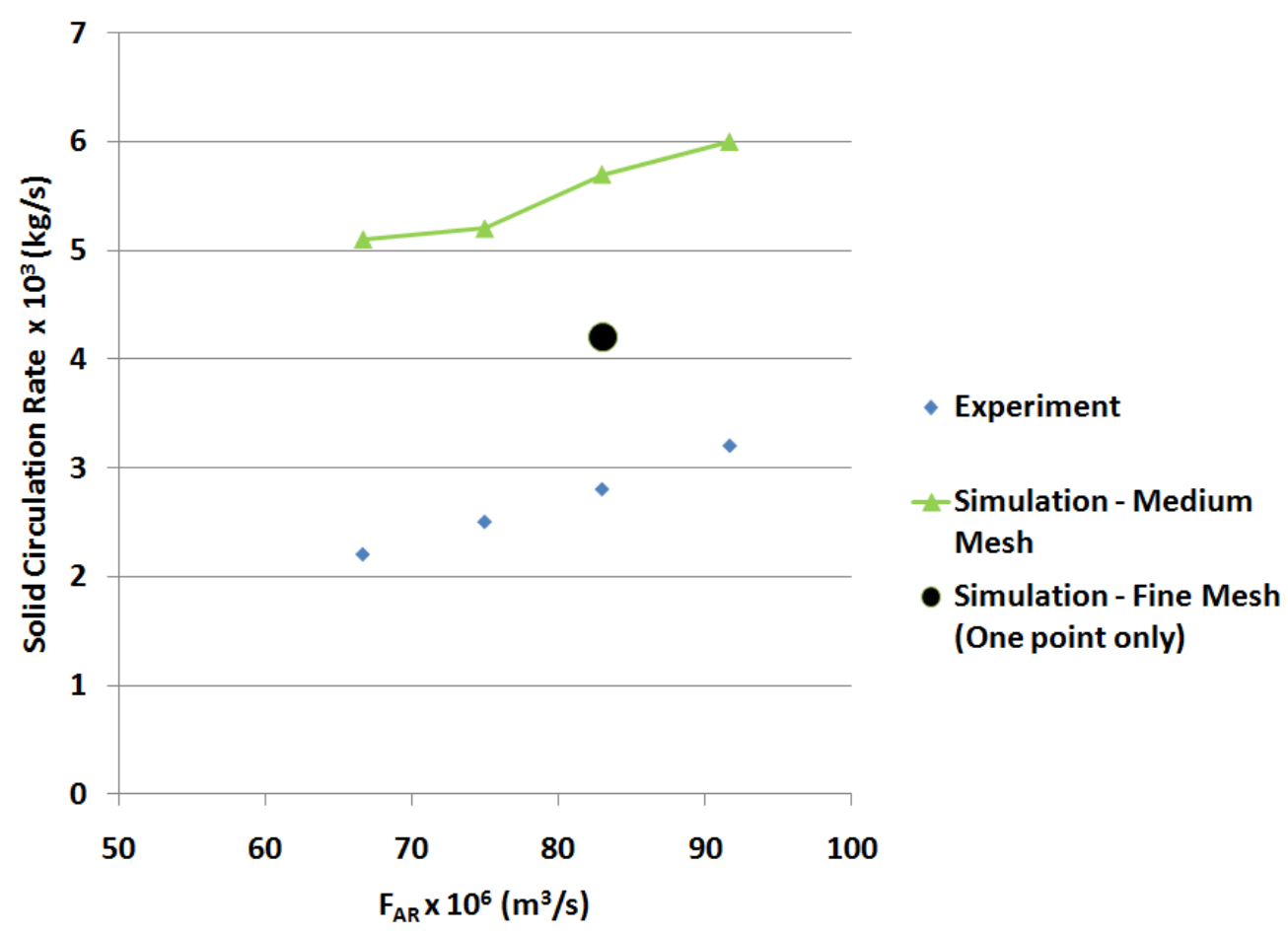

Fig. 9.13: Variation in solid circulation rate $\left(\mathrm{kg} / \mathrm{s} \times 10^{3}\right)$ with change in air reactor flow rates (AR flow rates vary from $66.7 \times 10^{-6}$ to $\left.91.7 \times 10^{-6} \mathrm{~m}^{3} / \mathrm{s}\right)$. FR $=5.8 \times 10^{-6} \mathrm{~m}^{3} / \mathrm{s}$. 


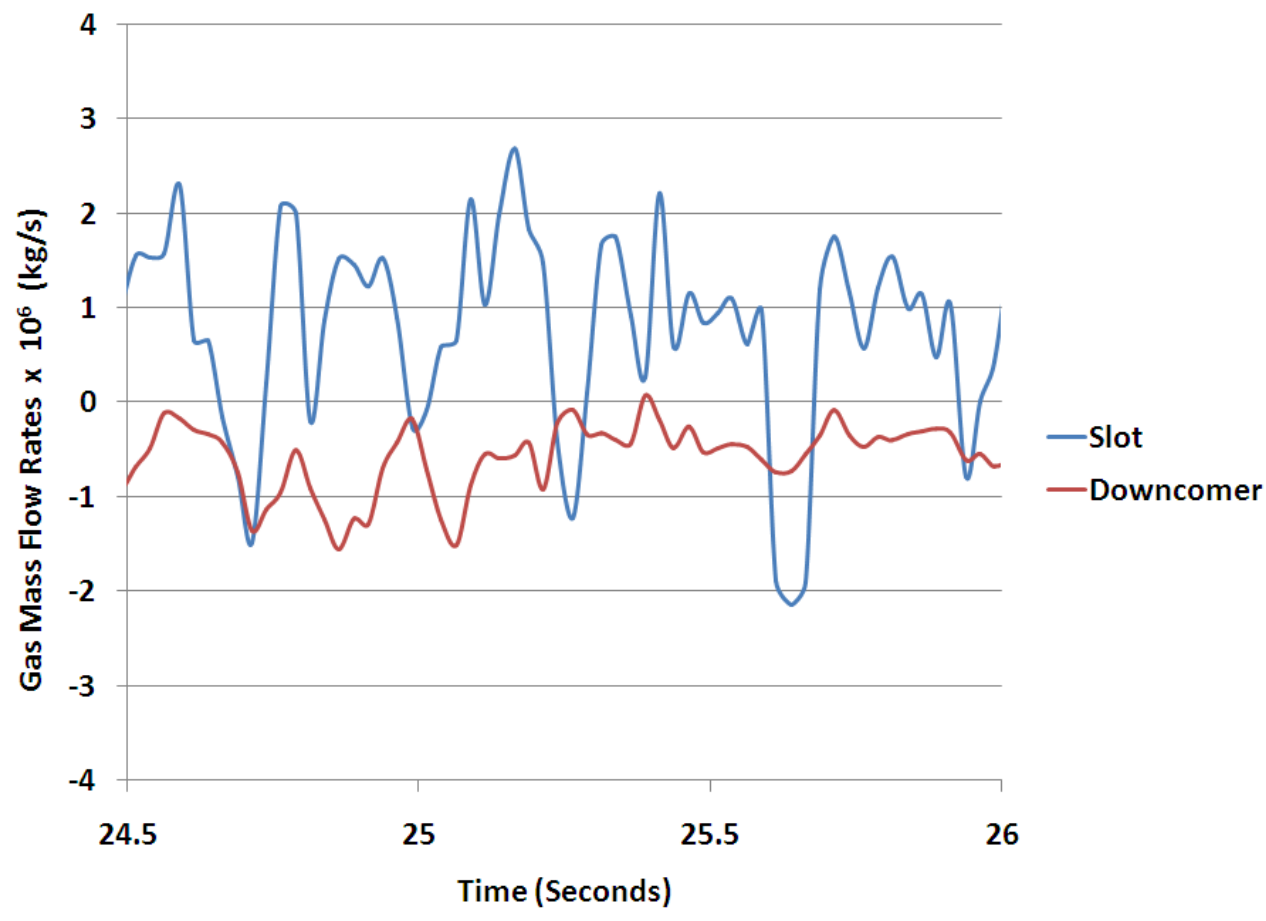

Fig. 9.14: Predicted gas flow rates $\left(\mathrm{kg} / \mathrm{s} \times 10^{6}\right)$ through the slot and downcomer. Air reactor flow rate is $83.0 \times 10^{-6} \mathrm{~m}^{3} / \mathrm{s}$. FR flow rate is $7.5 \times 10^{-6} \mathrm{~m} 3 / \mathrm{s}$. Positive mass flow rates indicate flow from FR to AR.

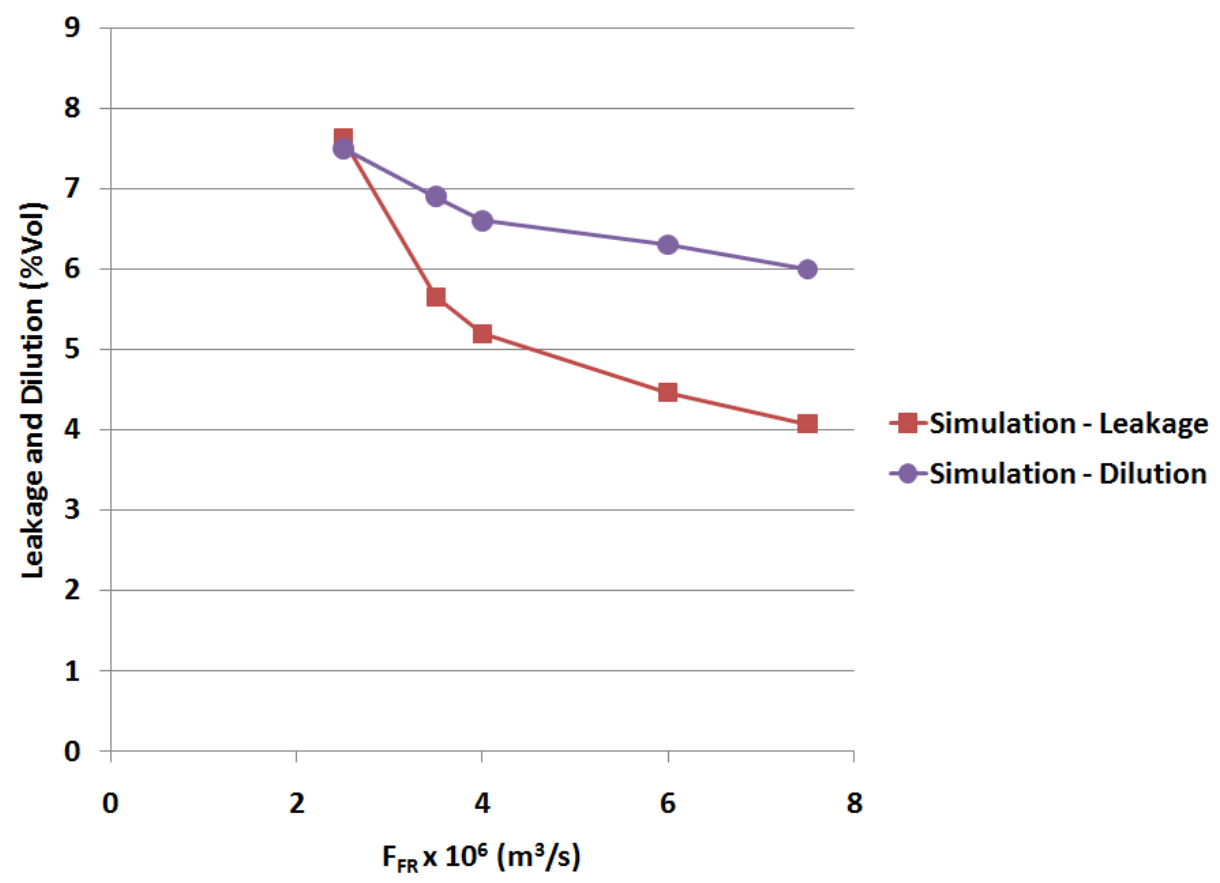

Fig. 9.15: Variation in leakage and dilution (\% Vol) with change in fuel reactor flow rates (FR flow rates vary from $2.5 \times 10^{-6}$ to $7.5 \times 10^{-6} \mathrm{~m}^{3} / \mathrm{s}$ ). AR flow rate was $83 \times 10^{-6} \mathrm{~m}^{3} / \mathrm{s}$. 


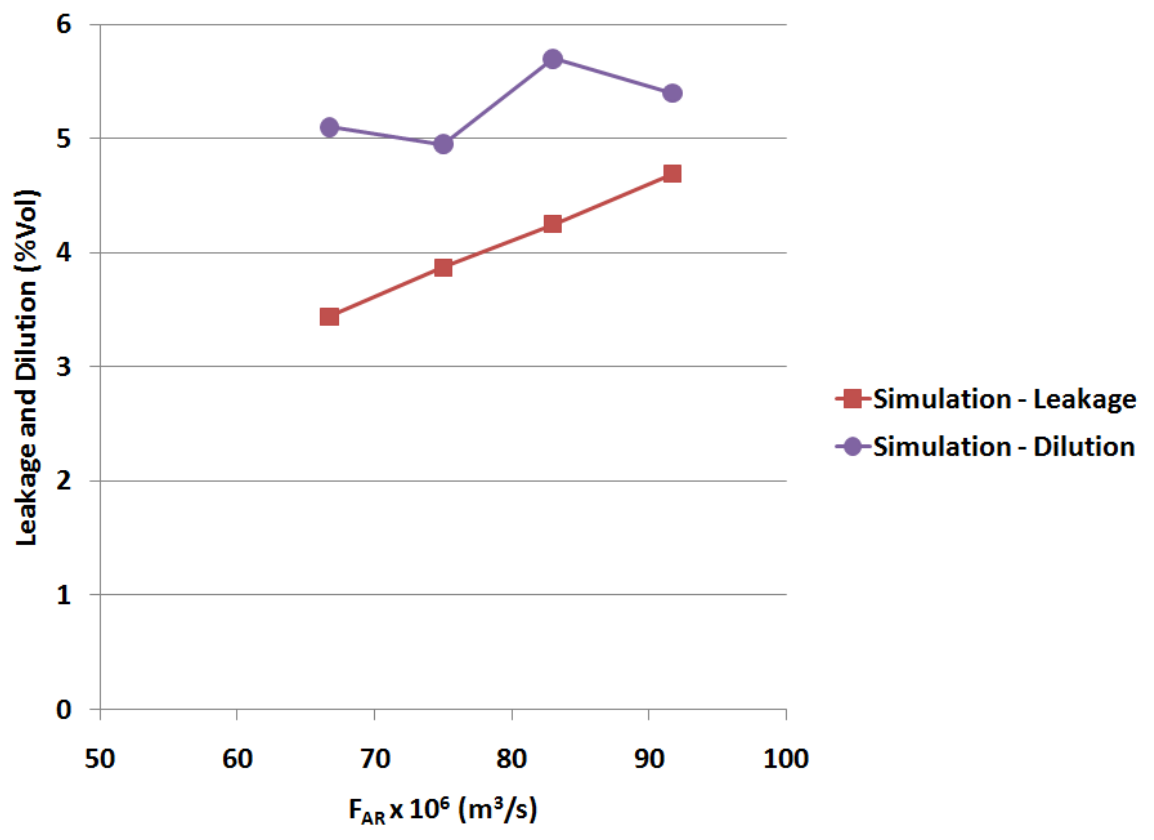

Fig. 9.16: Variation in leakage and dilution (\%Vol) with change in air reactor flow rates (AR flow rates vary from $66.7 \times 10^{-6}$ to $91.7 \times 10^{-6} \mathrm{~m}^{3} / \mathrm{s}$ ). FR flow rate was $5.8 \times 10^{-6} \mathrm{~m}^{3} / \mathrm{s}$.

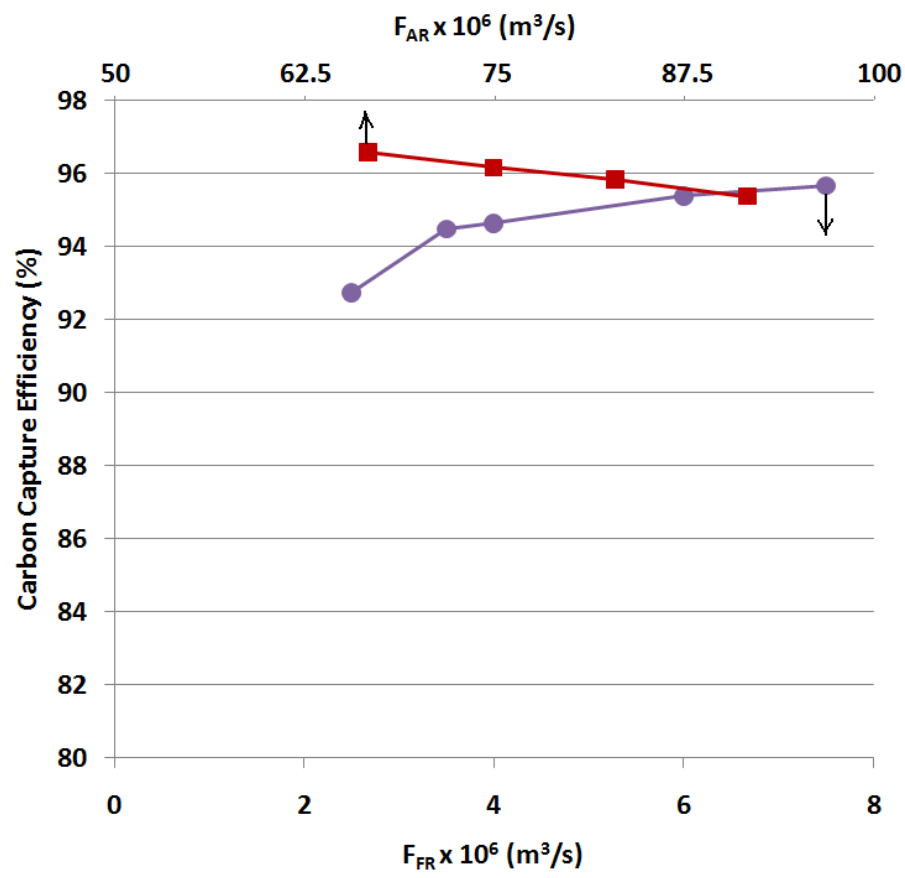

Fig. 9.17: Variation in carbon capture efficiency (\%) with change in fuel reactor and air reactor flow rates (FR flow rate varies from $2.5 \times 10^{-6}$ to $7.5 \times 10^{-6} \mathrm{~m}^{3} / \mathrm{s}$ and AR flow rates varies from $66.7 \times 10^{-6}$ to $\left.91.7 \times 10^{-6} \mathrm{~m}^{3} / \mathrm{s}\right)$. 


\subsection{Conclusion}

A CFD simulation for a complete chemical looping combustion system has been carried out. The solid particles have been modeled as a continuum fluid. Chemical kinetic models have been assembled from the literature for the reactions between the manganese oxide and fuel gases as well as air. The CFD model was used to simulate experiments of Abad et al., 2006. The model was able to predict the outlet concentrations of $\mathrm{CO}_{2}$, and $\mathrm{CH}_{4}$. The reactor performance at different operating temperatures was captured in a reasonable manner. It was found that the circulation of solids between the reactors is controlled by the frictional flow in the downcomer of the reactor, and hence predicting the correct amount of frictional energy dissipation was critical in obtaining accurate results. The performance of the reactor at different temperatures was also predicted accurately. The predicted gas leakage and dilution was within the observed variance reported by Abad et al. (2006). The solid mass distribution in the air and fuel reactors was predicted accurately. Also, the outlet concentrations of flue gases such as methane could be accurately predicted for a range of different reactor operating conditions. 


\section{Chapter 10: Design of Fuel Reactor utilizing Solid Fuel (Char)}

In previous chapters 7, 8 and 9, the capability of CFD models to predict the performance of CLC systems has been extensively documented, through comparisons with experimental results available in the literature. The focus of this portion of the study was to propose and evaluate possible fuel reactor designs for a chemical looping system utilizing solid fuel (char, as a simple model of coal). It would be desirable to utilize coal directly in the fuel reactor. In such a design, steam must be supplied as the fluidizing medium that converts the solid fuel to combustible gases such as $\mathrm{CO}$ and $\mathrm{H}_{2}$. The gaseous products of these reactions must then contact the hot, oxidized carrier resulting in conversion to $\mathrm{H}_{2} \mathrm{O}$ and $\mathrm{CO}_{2}$. Also, the reduced carrier particles must be returned to the AR for regeneration. It is critical that no unburned fuel, i.e., char, be returned with the spent carrier as this material will rapidly burn in the $A R$ and the resulting $\mathrm{CO}_{2}$ will escape capture. Special attention has been paid herein to Fe-based carriers (due to their low cost relative to other carriers).

A significant amount of research is currently underway in the design of CLC reactors, and several small scale (10 to $120 \mathrm{~kW}$ ) test plants have been prepared. However many of the CLC systems that have been studied utilize gaseous fuel (De Deigo et al., 2007; Son and Kim, 2006, Abad et al., 2006 etc.). Given the abundance of coal in the US, it is desirable to have CLC systems that can utilize coal. Before different designs are proposed and evaluated, there is a need to articulate the desirable features of a CLC system. A good chemical looping system should have the following characteristics:

1) High combustion efficiency: It is generally difficult to completely combust solid particles and achieve high combustion efficiency even when the coal particles are well mixed with metal oxides. This is because the combustible gases released by the coal at the top of the bed may not come into sufficient contact with metal oxide to burn completely.

2) Low leakage of solid coal/char particles from FR to AR: The time scale for gasification of coal at a temperature below the sintering temperature of practical metal oxide carriers is several minutes even when a high steam concentration is used ( $>50 \%)$. The time scales for a particle to move from one end of reactor to other are likely to be significantly shorter than this (especially for small scale reactors). Therefore there is considerable danger of coal particles leaking with the metal oxide into the AR and combusting there. This would reduce the overall $\mathrm{CO}_{2}$ capture efficiency. 
3) Effective mixing and heat transfer between hot solid particles and colder gases in fuel reactor: The solid particles coming in from AR should uniformly mix in the FR and maintain a uniform temperature in the fuel reactor.

4) Low solid inventory: The cost of producing suitable metal oxide particles for use in a CLC system can be high. However, low quantities of metal oxide inventory will likely result in incomplete combustion as well as inadequate heat transfer between reactors. Hence an optimum quantity of metal oxides will be desirable.

5) High solid circulation rates: For many metal oxides, such as iron oxide, the reactions in the FR are endothermic. Therefore, heat must be supplied to this reactor from the AR. The heat transfer generally takes place through the solid particles. To effectively transfer the heat from AR to FR, a high solid circulation rate is desired. Also the oxygen transfer from the air to fuel reactor takes place through the metal oxide or carrier. Hence, the solid circulation rates should also be high enough to supply enough oxygen to achieve a high combustion efficiency.

6) Low leakage of gases between air and fuel reactors: The combustible gases produced in the FR can leak through loop seals and combust in the AR. This does not affect the combustion efficiency of the process since the gases are still burnt, albeit in the wrong reactor. This is undesirable as $\mathrm{CO}_{2}$ will be released to the atmosphere. Similarly, if gas from the AR leaks into the FR then the outlet stream of flue gases from the FR will have a lower concentration of $\mathrm{CO}_{2}$. However, to achieve high carbon capture efficiency, this gas leakage has to be minimized. Moreover the presence of $N_{2}$ in the FR stream complicates the compression process during $\mathrm{CO}_{2}$ sequestration.

7) Small reactor size: It is desirable that the size of the CLC system be small and occupies the minimum amount of volume and floor space.

Before various designs for the FR are proposed a review of the performance of existing FR designs and their performance is needed. The next section provides these details.

\section{1: Performance of existing CLC systems utilizing solid fuels}

A limited number of batch, as well as continuous circulating fluidized bed CLC systems have been tested with solid fuels (Leion et al., 2007, 2008; Bergurand et al., 2008a, 2008b; Gao et al., 2008; Shen et al., 2009a, 2009b etc.). In this section the performance of several existing batch and continuous 
fluidized bed coal CLC systems published in the open literature are reviewed. To quantify the amount of un-burnt gases leaving the FR the \% Combustion and coal leakage are defined as:

$$
\% \text { Combustion }=\left(1-\frac{X_{C O}+X_{\mathrm{H}_{2}}+X_{\mathrm{CH}_{4}}}{X_{\mathrm{CO}_{2}}+X_{\mathrm{CO}}+X_{\mathrm{H}_{2}}+X_{\mathrm{CH}_{4}}}\right) \times 100
$$

where $X_{\mathrm{CO}}, X_{\mathrm{H}_{2}}, X_{\mathrm{CH}_{4}}$ and $X_{\mathrm{CO}_{2}}$ are the mole fractions of $\mathrm{CO}, \mathrm{H}_{2}, \mathrm{CH}_{4}$ and $\mathrm{CO}_{2}$ in the flue gas stream.

Coal Leakage $=\frac{\text { Coal mass flow rate at exit to FR }}{\text { Coal mass flow rate at inlet to FR }}$

Leion et al. $(2007,2008)$ studied the chemical-looping combustion of petroleum coke and South African coal using a batch reactor. The metal oxide used was $\mathrm{Fe}_{2} \mathrm{O}_{3}$. The batch reactor was alternated as air and FR. During the FR cycle, the coal was dropped into the reactor and the outlet flue gas concentrations were then measured. It was observed that the gasification products reacted rapidly with the metal oxides and the solid fuels were completely consumed within 20 minutes. The outlet flue gas stream consisted of relatively large percentages of un-burnt gases (such as $\mathrm{CO}, \mathrm{H}_{2}$ and $\mathrm{CH}_{4}$ ) in comparison with the $\mathrm{CO}_{2}$ concentration (Table 10.1). The percentage combustion was around 60 to $80 \%$, depending on the fuel used.

Berguerand and Lyngfelt (2008a, 2008b) designed a 10kW continuous operating circulating fluidized bed chemical looping combustor for solid fuels, and tested with petcoke and South African coal. The metal oxide used was $\mathrm{Fe}_{2} \mathrm{O}_{3}$. To prevent the leakage of coal particles from the FR to AR, they utilized a carbon stripper mechanism which separated the unreacted coal particles from the metal oxides. This ensured that primarily metal oxides were circulated back to AR. Despite the use of a complex carbon stripper mechanism to prevent coal leakage to the $A R$, the carbon capture efficiencies were in the range of $75 \%$ to $85 \%$ on average. This indicates that a significant amount of coal might have leaked into the AR. Also the percentage combustion (gas stream consisting of un-burnt gases like $\mathrm{CO}, \mathrm{H}_{2}$ and $\mathrm{CH}_{4}$ ) was between 70 to $80 \%$.

Gao et al. (2008) carried out experiments for CLC with coal as fuel and Nickel oxide as the carrier in a fluidized bed. The batch reactor was alternated as air and FR. The percentage combustion was close 
to $90 \%$ (Table 10.1). Their carbon capture efficiency was not reported. Shen et al. (2009a and 2009b) carried out experiments for CLC with biomass and coal as fuel. Iron oxide was used as an oxygen carrier in their $10 \mathrm{~kW}$ continuous reactor of interconnected fluidized beds. The percentage combustion was above $90 \%$, but this did not include the $\mathrm{H}_{2}$ concentration as it was not measured (Table 10.1). Also the reported carbon capture efficiency varied between 72 to $80 \%$ depending on the operating temperature.

The difficulties in achieving high combustion efficiencies that are close to $100 \%$ is partly because of the insufficient time for combustible gases released by coal particles floating on top of the bed to react with the metal oxide. Also, the reason for low carbon capture efficiencies primarily stems from the inability to prevent coal particles from being dragged to the AR along with the metal oxides. From the above discussion it is clear that many of the experimental studies show considerable promise. However, there is still a significant amount of reactor design and development that will be required to reach a high percentage combustion as well as achieve carbon capture efficiencies of more than $90 \%$, as stipulated by the United States Dept of Energy (USDOE). CFD can be used as a design tool to help develop efficient CLC fuel reactors that will meet these requirements.

Table 10. 1: Carbon capture efficiency and percentage combustion in some published experimental studies.

\begin{tabular}{|c|c|c|c|c|c|c|c|c|c|}
\hline Experiment & Type & Fuel & $\begin{array}{l}\text { Metal } \\
\text { Oxide }\end{array}$ & $\begin{array}{l}X_{\mathrm{CO} 2} \\
(\%)\end{array}$ & $\begin{array}{l}X_{C O} \\
(\%)\end{array}$ & $\begin{array}{c}X_{H 2} \\
(\%)\end{array}$ & $\begin{array}{l}X_{C H 4} \\
\text { (\%) }\end{array}$ & $\begin{array}{c}\% \\
\text { Combu } \\
\text { stion }\end{array}$ & $\begin{array}{c}\text { Carbon } \\
\text { Capture } \\
\text { efficiency }\end{array}$ \\
\hline $\begin{array}{l}\text { Leion et al., } \\
2007\end{array}$ & Batch & Petcoke & $\mathrm{Fe}_{2} \mathrm{O}_{3}$ & 5 & 0.5 & 0.3 & 0.5 & 79.4 & - \\
\hline $\begin{array}{l}\text { Leion et al., } \\
2008\end{array}$ & Batch & Coal & $\mathrm{Fe}_{2} \mathrm{O}_{3}$ & 5 & 1 & 1 & 0.5 & 66.7 & - \\
\hline $\begin{array}{l}\text { Berguerand } \\
\text { and } \\
\text { Lyngefelt, } \\
2008\end{array}$ & Continuous & Petcoke & $\mathrm{Fe}_{2} \mathrm{O}_{3}$ & 17 & 2 & 4 & 1 & 70.8 & 75 \\
\hline $\begin{array}{l}\text { Berguerand } \\
\text { and } \\
\text { Lyngefelt, } \\
2009\end{array}$ & Continuous & Coal & $\mathrm{Fe}_{2} \mathrm{O}_{3}$ & 17 & 3 & NA & 1.5 & 79.1 & 85 \\
\hline $\begin{array}{l}\text { Gao et al., } \\
2008\end{array}$ & Batch & Coal & $\mathrm{NiO}$ & 90 & 7 & 2 & 1 & 90 & - \\
\hline $\begin{array}{c}\text { Shen et al., } \\
2009\end{array}$ & Continuous & Coal & $\mathrm{NiO}$ & 94 & 5 & NA & 1 & 94 & 78 \\
\hline $\begin{array}{c}\text { Shen et al., } \\
2009\end{array}$ & Continuous & Biomass & $\mathrm{Fe}_{2} \mathrm{O}_{3}$ & 82 & 6 & NA & 2 & 91.1 & - \\
\hline
\end{tabular}




\section{2: Problem Statement}

The goal of the design portion of the current study is to suggest suitable designs for the FR of a $2 \mathrm{KW}$ experimental chemical looping reactor that can provide high combustion efficiency as well as high carbon capture, and make predictions about their performance using CFD. Due to the considerable difficulties in fully modeling coal conversion, the present design study has been limited to the use of char. The char has been assumed to be a combination of $85 \%$ carbon and $15 \%$ ash. There are a large number of desirable characteristics of a CLC system as elaborated earlier. The present study was limited to the FR with the following aspects being studied:

1) Combustion efficiency of the FR,

2) Carbon capture efficiency,

3) Amount of solid inventory,

4) Size or space required for the FR.

Reactor Power Rating (heat generated) was set at $2 \mathrm{~kW}$ as this is achievable in a modest size laboratory scale experimental reactor that can be developed in a research lab, such as that at National Energy Technology Laboratory, (the funding agency of the present study). The metal oxide to be utilized was assumed to be iron oxide because of its low cost and relative abundance.

The particle sizes were selected based on following constraints.

- The metal oxide particles should be light enough to allow their transport in the AR.

- There should be enough weight difference between char and metal oxide particles to allow for their segregation in the FR. This segregation of char is essential to prevent its leakage to the AR due to drag from the metal oxide particles.

Based on the above criteria, the metal oxide particles were selected to be of 200 micron size and density of $3500 \mathrm{~kg} / \mathrm{m}^{3}$. The char particles were selected to be 100 micron size and density of $1500 \mathrm{~kg} / \mathrm{m}^{3}$.

The following values were determined from the spread sheet model described in Chapter 3 and section 3.2.

- Char flow rate (carbon $=85 \%$ and ash $=15 \%)=7.8 \times 10^{-5} \mathrm{~kg} / \mathrm{s}$

- Metal oxide flow rate $=0.08 \mathrm{~kg} / \mathrm{s}$

- Steam flow rate $=3 \times 10^{-4} \mathrm{~kg} / \mathrm{s}$ ( 3 times the stoichiometric amount).

- Steady state char mass in FR $=0.02 \mathrm{~kg}$ 
- Total mass of metal oxide used $=20 \mathrm{~kg}$ (12.5 times stoichiometric mass required to burn char)

- $\quad$ Assumed temperature of $A R=1250 \mathrm{~K}$

- Calculated temperature of $F R=1215 \mathrm{~K}$

- Estimated minimum flow cross-sectional area required for the $F R=194 \mathrm{~cm}^{2}$

- Estimated minimum flow cross-sectional area required for the $A R=16.1 \mathrm{~cm}^{2}$

\section{The Reaction Scheme and Rates}

In the present study, char was used as the fuel, and it was assumed to be a combination of $85 \%$ carbon and $15 \%$ ash. The following reactions were modeled in the simulation:

Char Gasification by $\mathrm{H}_{2} \mathrm{O}$

$$
\mathrm{Char}+\mathrm{H}_{2} \mathrm{O} \rightarrow \mathrm{CO}+\mathrm{H}_{2}
$$

Char Gasification by $\mathrm{CO}_{2}$

$$
\mathrm{Char}+\mathrm{CO}_{2} \rightarrow 2 \mathrm{CO}
$$

Metal Oxide Reduction

$$
\begin{aligned}
& 3 \mathrm{Fe}_{2} \mathrm{O}_{3}+\mathrm{CO} \rightarrow 2 \mathrm{Fe}_{3} \mathrm{O}_{4}+\mathrm{CO}_{2} \\
& 3 \mathrm{Fe}_{2} \mathrm{O}_{3}+\mathrm{H}_{2} \rightarrow 2 \mathrm{Fe}_{3} \mathrm{O}_{4}+\mathrm{H}_{2} \mathrm{O}
\end{aligned}
$$

Water-Gas-Shift Reaction

$$
\mathrm{CO}+\mathrm{H}_{2} \mathrm{O} \rightarrow \mathrm{CO}_{2}+\mathrm{H}_{2}
$$

\section{$\underline{\text { Reaction Rates: }}$}

The char gasification rates given by Everson et al. (2006) have been used in the present study. In general the gasification rates of various types of coals can vary significantly (by more than an order of magnitude). The coal gasification rates based on Everson et al. (2006), as described in Chapter 8, have been used in this study. Reaction rates for metal oxide reduction and water gas shift are also described in Chapter 8. Section 8.5 and 8.6 provide details for these rates.

\section{Density Variation of Char:}

At the lower temperatures characteristic of fluidized beds $(T<1300 \mathrm{~K})$, the gasification reactions being slow, it is thought that the reacting gas has enough time to penetrate through the pores of the 124 
char particle and the reactions take place throughout the volume of the particle (Syamlal and Bissett, 1992). Based on this reasoning, it has been assumed that the particle diameter is constant, but the density is a linear function of conversion, $X$ :

$$
\rho_{P}=\left\{\begin{array}{cl}
\rho_{P, \text { max }}(1-X) & X<X_{c} \\
\rho_{P, \text { min }} & X>X_{c}
\end{array}\right.
$$

The density of the unburned char, $\rho_{P, \max }$, is assumed to be $1500 \mathrm{~kg} / \mathrm{m}^{3}$. The density has been limited to a minimum value, $\rho_{P, \text { min }}=100 \mathrm{~kg} / \mathrm{m}^{3}$.

\section{3: Different fuel reactor designs}

Three different designs, as described below, have been developed and analyzed with the goal being to obtain high combustion efficiency and high carbon capture.

Design 1: This design uses a simple cylindrical geometry to combust the char. The metal oxide and char are fluidized at velocities higher than the minimum fluidization to allow good mixing of char and metal oxide (See section 10.3.1 for details).

Design 2: In this case the FR is divided into two sections. The char is segregated in section 1 and allowed to mix with the metal oxides in section 2 . The segregation of char in section 1 prevents the leakage of char particles to the AR resulting in higher carbon capture efficiency (See Fig. 10.6).

Design 3: This design consists of a multi compartment FR where the char particles are isolated and gasified in conjunction. Then the gasification products such as $\mathrm{CO}$ and $\mathrm{H}_{2}$, are made to react with hot metal oxides in separate compartments. In the present design there are a total of four compartments.

\subsection{1: Details of Design 1:}

This design uses a simple cylindrical vessel where the superficial gas velocities are well above minimum fluidization velocity of the particles so that the char and metal oxide are well mixed. Given that the char in the FR is likely to be present in widely varying density and size, a reactor providing good mixing is essential. If the lighter char particles segregate to the top then the combustible gases released 
from them will not react with the metal oxide thus providing a low percentage combustion. Fig. 10.1 shows the overall working of the design as well as the major dimensions of the proposed reactor. The metal oxide inventory used for the simulations was $20 \mathrm{~kg}$.

A 2D planar formulation was used to represent the reactor in the CFD simulation. The cell count for the grid was 13852. Higher order discretization schemes were used. The spatial discretization scheme for volume fraction equations was quadratic upwind interpolation scheme (QUICK). A second order upwind discretization was used for all other equations. The temporal discretization scheme in all cases was first order implicit.

Fig 10.2 shows an example of the volume fractions of the metal oxide and the char phase at one time instant well after the initial startup transience. The two solid phases appear to be reasonably well mixed. Bubble formation can be clearly observed. Also the bed height is limited by the outflow location of the metal oxide (Also see Fig. 10.1).

Fig. 10.3a shows a sample contour plot of steam mole fraction at the same time instant. The steam concentrations are high at the inlet (100\%) and decrease rapidly moving upwards through the bed. The decrease in steam concentration is due to gasification of the char particles. The bubbles have a slightly higher concentration of steam than the surrounding emulsion region which indicates that some of the steam bypasses the bed through the bubbles. Fig. 10.3b shows a mole fraction plot of $\mathrm{CO}$. The $\mathrm{CO}$ concentration is low at the inlet region but increases with bed height as the steam reacts with the char. Also the $\mathrm{CO}$ concentrations are high in the emulsion region where it forms due to gasification. From Fig. $10.3 \mathrm{c}$ it can be observed that the $\mathrm{CO}_{2}$ concentrations also increase as the bed height increases. This is primarily because of reaction of $\mathrm{CO}$ with the metal oxides resulting in formation of $\mathrm{CO}_{2}$. Also the concentration of $\mathrm{CO}_{2}$ is higher in the emulsion phase and low in bubbles. This can be expected as the $\mathrm{CO}$ is primarily formed in the emulsion region of the bed.

Figs. 10.4 and 10.5 show the computed metal oxide and carbon mass flow rates versus time for a 5 second time period at the exit location. The mass loss occurs in periodic spikes generally because of eruption of bubbles near the exit. Table 10.2 provides an average leakage rate and the percentage combustion. High combustion efficiencies, greater than $90 \%$, can be achieved with this design, but the char leakage rates are also high at $67 \%$ which will result in low carbon capture efficiency. 
Design 1 has two important limitations:

1) Generally the time scale for gasification of char is large (several minutes) even when a high steam concentration is used ( $>50 \%)$. The time scales of particle motion, especially in smaller scale experimental reactors, are likely to be significantly smaller than this. Therefore a considerable amount of char particles leak with the metal oxide into the $A R$ and combust there. This is undesirable as $\mathrm{CO}_{2}$ will then leak into the atmosphere.

2) It is difficult to completely consume combustible gases released from solid fuels even when the char and metal oxides are completely mixed. This is primarily because combustible gases released from char particles near the top of the bed do not have enough contact time with the metal oxide to be completely consumed. Therefore, it may be necessary to have a more complex FR design to combust the char effectively.

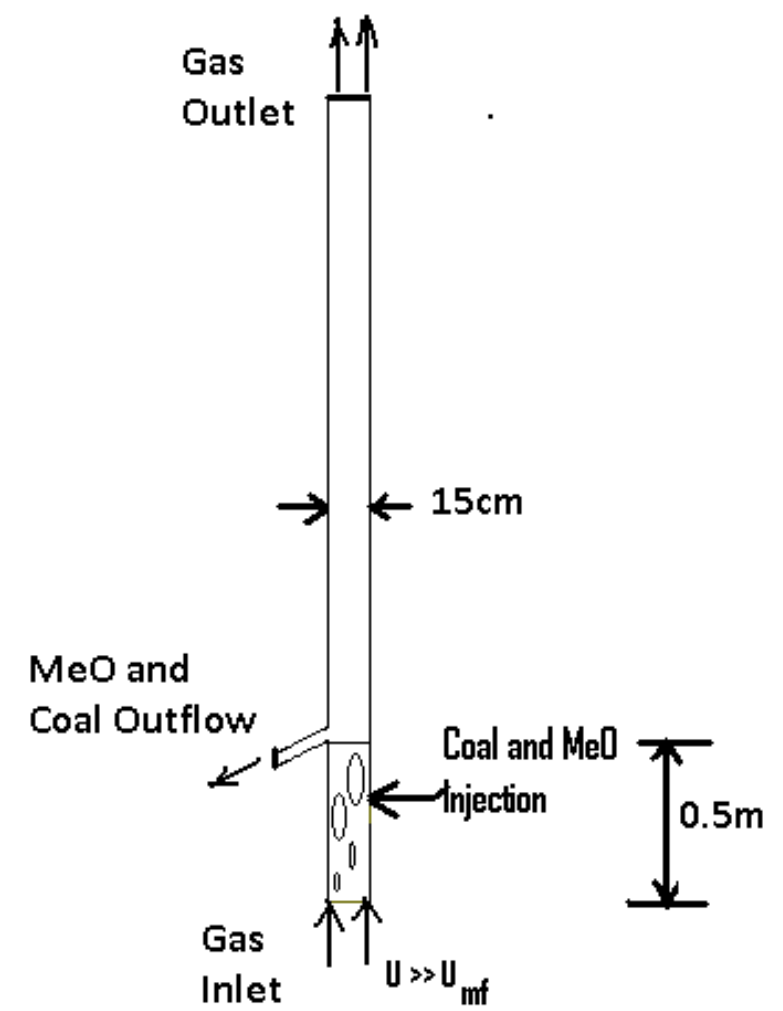

Fig. 10.1: Design 1 for the FR utilizing char as fuel. 


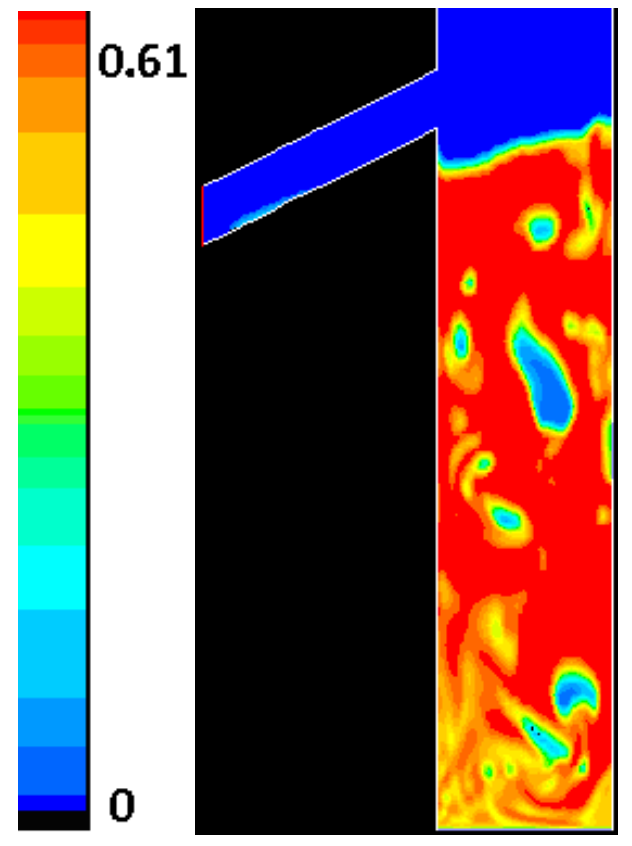

a) $\mathrm{MeO}$

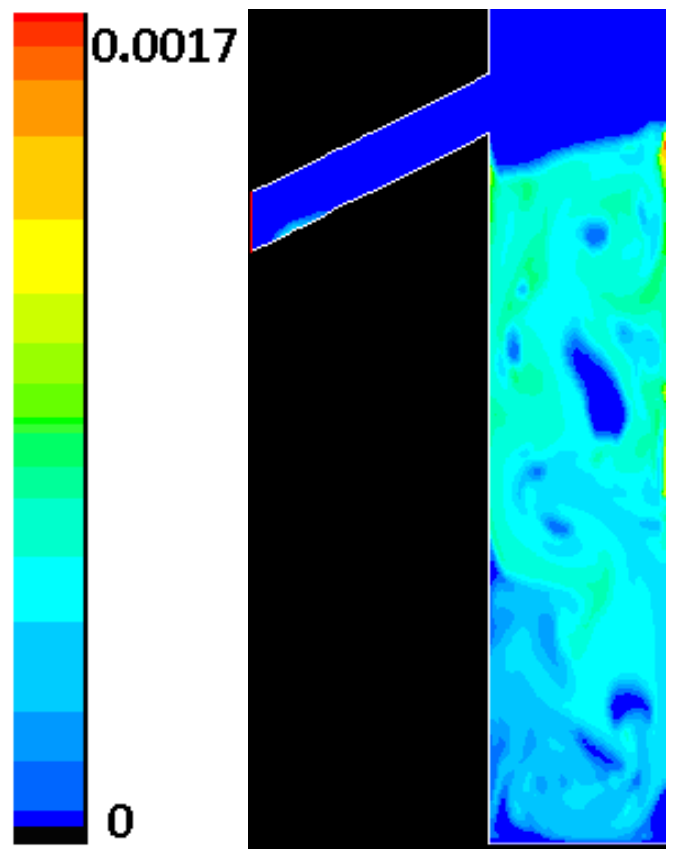

b) Char

Fig. 10.2: Instantaneous volume fraction, $20 \mathrm{~kg}$ metal oxide inventory ( 100 seconds of simulation time).

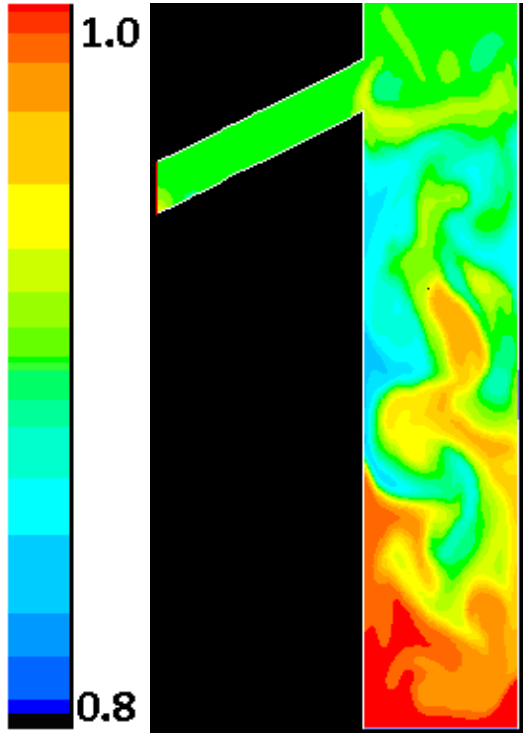

a) $\mathrm{H}_{2} \mathrm{O}$

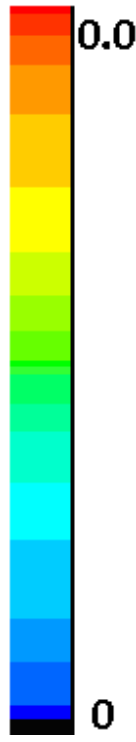
(1)

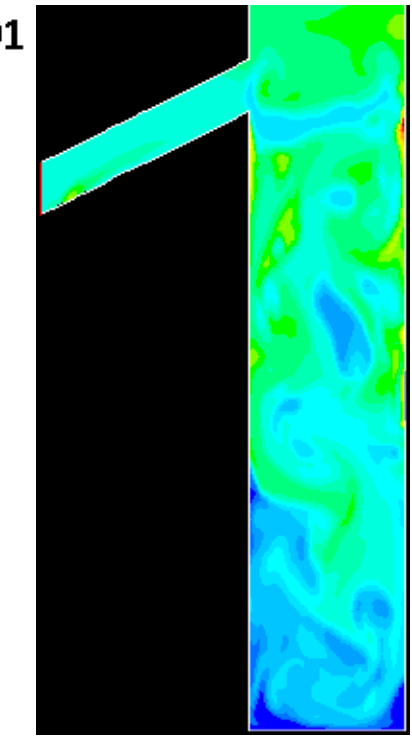

b) $\mathrm{CO}$
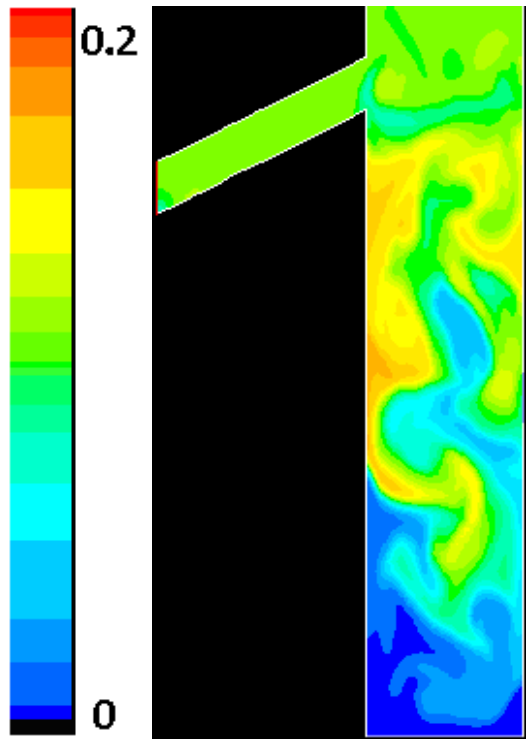

c) $\mathrm{CO}_{2}$

Fig. 10.3: Mole fraction of flue gases for Design 1 ( 100 seconds of simulation time). 


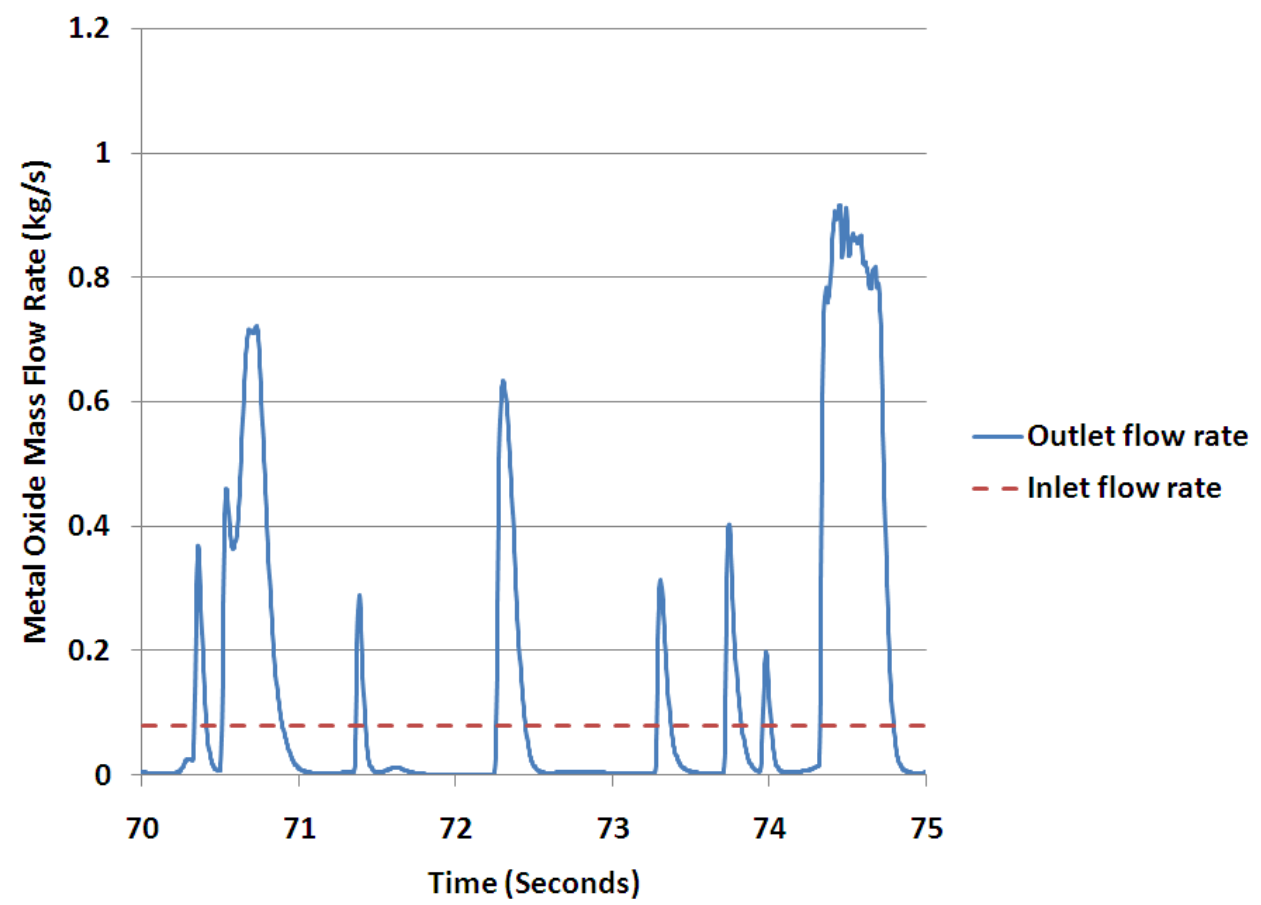

Fig. 10.4: Time varying metal oxide outlet mass flow rate for Design 1, $20 \mathrm{~kg}$ inventory ( 75 seconds of simulation time).

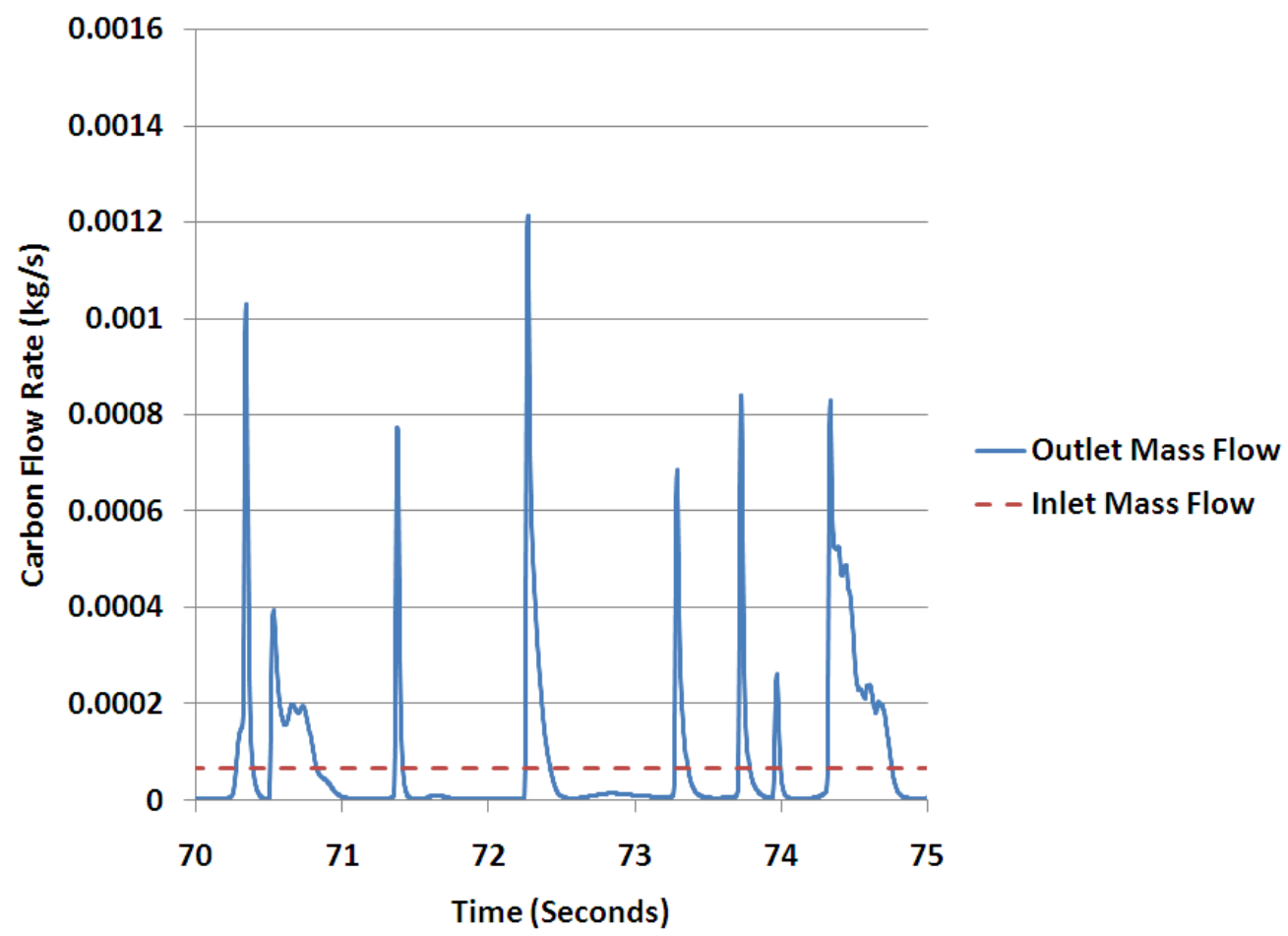

Fig. 10.5: Time varying carbon outlet mass flow rate for Design 1, $20 \mathrm{~kg}$ inventory ( 75 seconds of simulation time). 
Table 10.2: Comparison of char leakage and percentage combustion for Design 1.

\begin{tabular}{|l|l|l|l|l|l|}
\hline & $\begin{array}{l}\text { Char } \\
\text { Leakage }\end{array}$ & $X_{\mathrm{CO}}$ & $X_{\mathrm{H} 2}$ & $X_{\mathrm{CO} 2}$ & $\begin{array}{l}\text { Combustion } \\
\text { Com }\end{array}$ \\
\hline Design 1 & $67.36 \%$ & 0.00048 & 0.00012 & 0.1 & $99.4 \%$ \\
\hline
\end{tabular}

\subsection{2: Details of Design 2:}

As discussed above, the Design 1 provides combustion efficiency in excess of $90 \%$ but also has a high leakage of char particles from the AR to the FR which is unacceptable. To prevent char leakage it is essential that some mechanism to segregate the char particles from the metal oxide be implemented. In Design 2 this segregation is achieved by dividing the reactor into two sections as shown in Fig. 10.6.

Section 1: This is a low velocity moving bed with superficial velocity just equal to the minimum fluidization velocity of the heavy metal oxide particles. Since the char particles are lighter than the metal oxide they have a natural tendency to segregate and float on top. The low velocity in section 1 prevents bubble formation which is primarily responsible for the mixing of char and $\mathrm{MeO}$ particles. Because of the low fluid velocity and absence of bubbles, the lighter char particles that enter section 1 have a tendency to float to the top of the bed.

Section 2: This is high velocity bubbling bed that mixes the char and MeO. Good mixing of char and metal oxide is essential for the complete combustion of char.

In the present simulations the metal oxide particles are assumed to be of a fixed size. However in actual practice there would be some particle size distribution associated with the metal oxide particles. Also, during the operation there is likely to be some attrition adding to the variation of particle size. The size distribution of particles could affect the fluidization behavior of the reactor and alter the minimum fluidization velocity of the metal oxide. The CFD model used in the present study does not account for information about particle size and shape distributions. This can cause significant differences near minimum fluidization conditions where small changes in this velocity can change the fluidization behavior from a moving bed to a bubbling bed. 
To analyze the working of this reactor both 2D-Axisymmetric and 3D simulations were carried out (Fig. 10.7). Previous 2D-Axisymmetric simulations of solid fuels presented in chapter 8 showed good comparisons between the predicted results and the experiments. The 3D simulations take a significantly longer amount of time in comparison with 2D simulations. Due to the high computational costs, the 3D calculations have been restricted to one simulation. The cell count for the 2D-Axisymmetric grid was 10328 and for the 3D simulations it was 110000. Higher order discretization schemes were used. The spatial discretization scheme for the volume fraction equations was quadratic upwind interpolation scheme (QUICK). A second order upwind discretization was used for all other equations. The temporal discretization scheme in all cases was first order implicit.

The Design 2 is simple to construct and prevents large scale leakage of char to the AR. Because of this, Design 2 was considered to be of interest to NETL for building their experimental facility. Therefore, a study on the effects of the following parameters on the percentage combustion and char leakage was carried out:

reactor diameter/size,

solid inventory in section 2 of the reactor, and

cone angle at the bottom of the section 2 .

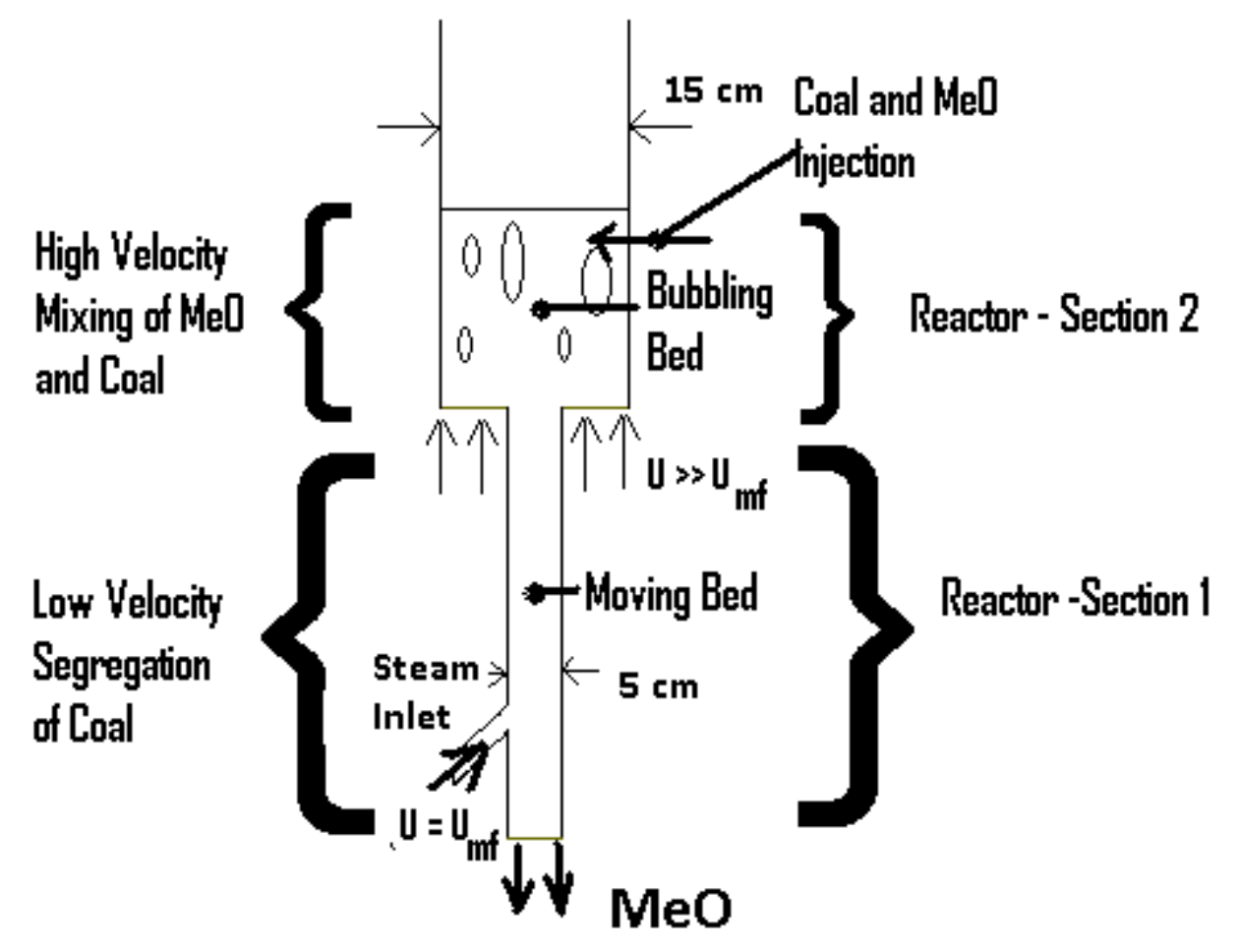

Fig. 10.6: Design 2 for a char-metal oxide test FR. 


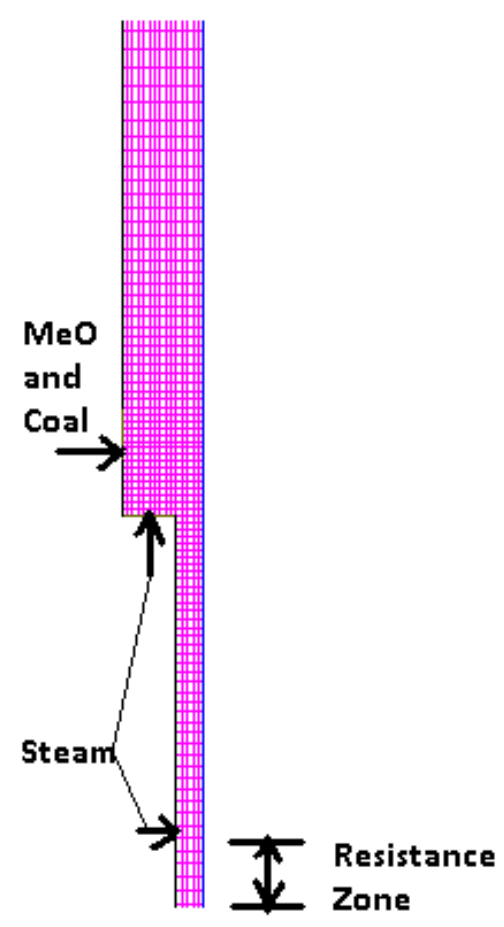

a) 2D-Axisymmetric mesh

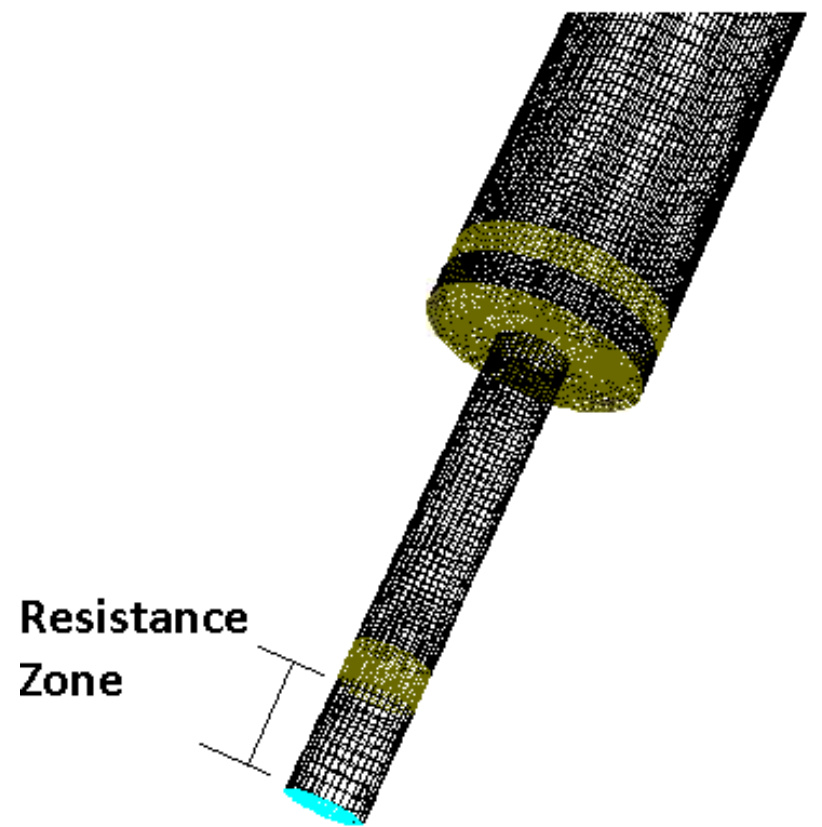

b) 3D Mesh

Fig. 10.7: 2D and 3D mesh used in the analysis.

\section{Effect of Reactor Size:}

Fig. 10.8 shows two different reactor sizes tested. In case 2 the diameter of the reactor was double that of case 1 . In both cases the solids inventory in section 2 of the reactor (region where combustion occurs) is around $20 \mathrm{~kg}$.

Table 10.3 provides the comparison of the predicted char leakage and \% combustion for case 1 and case 2. The char leakage is significantly less in case 2, although for both cases the leakage is significantly less than in Design 1 . This is primarily because of the lower solids velocity that exists in section 1 of case 2 . The desired mass flow rate of metal oxide to supply enough oxygen and heat is fixed at $0.08 \mathrm{~kg} / \mathrm{s}$ (based on the spread sheet model, chapter 3 ). Therefore increasing the cross-sectional area of section 1 of the reactor will slow down the metal oxide velocity considerably.

$v_{\text {solid }}=\frac{\dot{m}}{\rho_{s} \varepsilon_{s} A}$

A lower metal oxide velocity would mean that less char is dragged along with the metal oxides. 


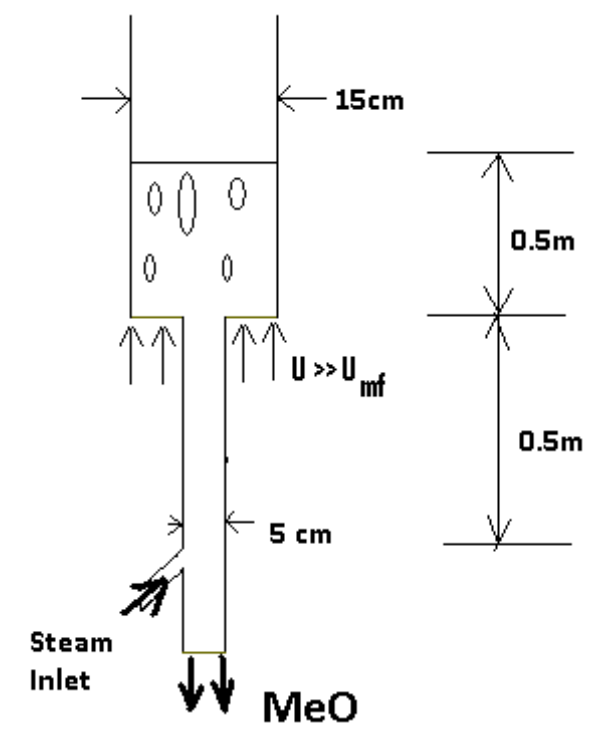

a) Case 1

(Section $1 \mathrm{dia}=5 \mathrm{~cm}$; Section $2 \mathrm{dia}=15 \mathrm{~cm}$ )

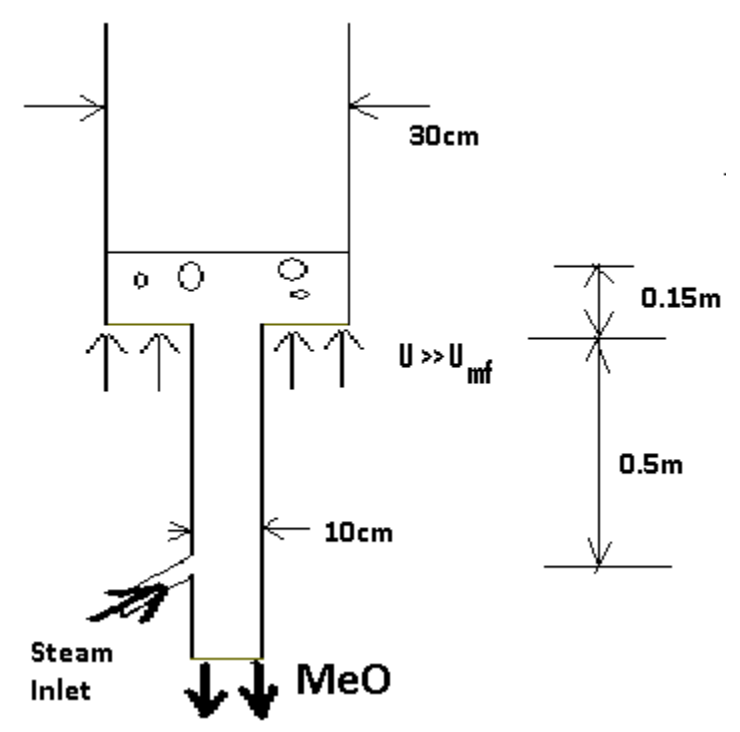

b) Case 2

(Section $1 \mathrm{dia}=10 \mathrm{~cm}$; Section $2 \mathrm{dia}=30 \mathrm{~cm}$ )

Fig. 10. 8: Two different reactor sizes tested. Diameters in Case 2 are double of Case 1. The mass in section 2 was constant at around $21 \mathrm{~kg}$.

The exit mole fraction of $\mathrm{CO}_{2}$ in case 2 is lower (Table 10.3). This is primarily because the steam flow rates are higher. The fluidizing velocity in section 2 is fixed at $0.1 \mathrm{~m} / \mathrm{s}$ for both cases. Since case 2 has a higher cross-sectional area, it results in higher steam flow rates. The combustion efficiency in case 2 is slightly less than in case 1 ; this is primarily because of the shorter residence time of combustible gases in the bed as well as greater percentage of char mass floating on top of the bed.

Table 10.3: Comparison of char leakage and percentage combustion for case 1 and case 2.

\begin{tabular}{|c|c|c|c|c|c|}
\hline & $\begin{array}{l}\text { Char } \\
\text { Leakage }\end{array}$ & $X_{C O}$ & $\mathrm{X}_{\mathrm{H} 2}$ & $\mathrm{X}_{\mathrm{CO} 2}$ & $\begin{array}{l}\% \\
\text { Combustion }\end{array}$ \\
\hline Case 1 & $21.86 \%$ & 0.0061 & 0.0029 & 0.32 & $97.26 \%$ \\
\hline Case 2 & $5.70 \%$ & 0.012 & 0.007 & 0.11 & $85.27 \%$ \\
\hline
\end{tabular}

Fig. 10.9 shows examples of the computed metal oxide volume fraction. Well resolved bubble formation is observed in the $2 \mathrm{D}$ simulations (Figs. 10.9a and 10.9b). The metal oxide volume fraction 
from the 3D simulation for case 2 is shown in Fig. 10.9c. The 3D simulations show highly diffused flow features primarily because of the coarse mesh used. The 3D simulations have been restricted to a coarse mesh because of the high computational cost.

Fig. 10.10 shows examples of the computed char volume fraction. From Fig. 10.10a, it is clearly observed that there is a large char fraction in section 1 of the FR (Char should be primarily in section 2). This confirms the high leakage tendency in case 1. From Fig. 10.10b (2D simulation) and Fig. 10.10c (3D simulation), a clear accumulation of char at the top-center of the bed can be observed (for case 2 ). This is primarily because this region experiences the least amount of disturbance created due to bubble motion. The bubbles are primarily responsible for the mixing of lighter char particles and the heavier metal oxides. The bubbles are formed primarily due to the high velocity outer annular region in section 2 and, therefore, the effect of bubbles on the central core of the reactor is less. This creates a tendency for the particles in the central core region of the reactor to remain unmixed. The tendency of the char particles to accumulate in the top-center of the bed will result in large amount of combustible gases (CO and $\mathrm{H}_{2}$ ) to be released through exhaust gases and this will drive down the percentage combustion. If the bed height is lower and diameter of the central section 1 is large then there will be less mixing of char particles in the top-center region of the bed and this will decrease the combustion efficiency of the reactor.

\section{Effect of solid inventory in section 2 of the reactor}

The solid inventory in section 2 is primarily responsible for the reaction with combustible gases. Hence its effect has to be understood. Table 10.4 provides the comparison of the predicted leakage and $\%$ combustion for case 1 as the solid inventory is increased. As the solid inventory increases there is lower char leakage. This is primarily because of lower char concentration per unit mass of metal oxide. A lower char concentration results in less amount of char being dragged with the metal oxide into section 1. From Table 10.4, as the solid inventory is increased there also is an increase in combustion efficiency in general. This should be expected as a greater amount of metal oxide is available. However, the increase from $10 \mathrm{~kg}$ to about $20 \mathrm{~kg}$ produces only a marginal gain of around 2\%. Figs. 10.11 and 10.12 show examples of the $\mathrm{MeO}$ and char volume fraction at bed inventory levels of $10 \mathrm{~kg}$ and $5 \mathrm{~kg}$, respectively. At smaller bed height/inventory level, there is a greater tendency for char to float on the top-center region of the bed. This is expected to contribute to the lower combustion efficiencies. Also 
the lower bed heights will result in less time for contact between combustible gases and metal oxides resulting in a lower percentage combustion.
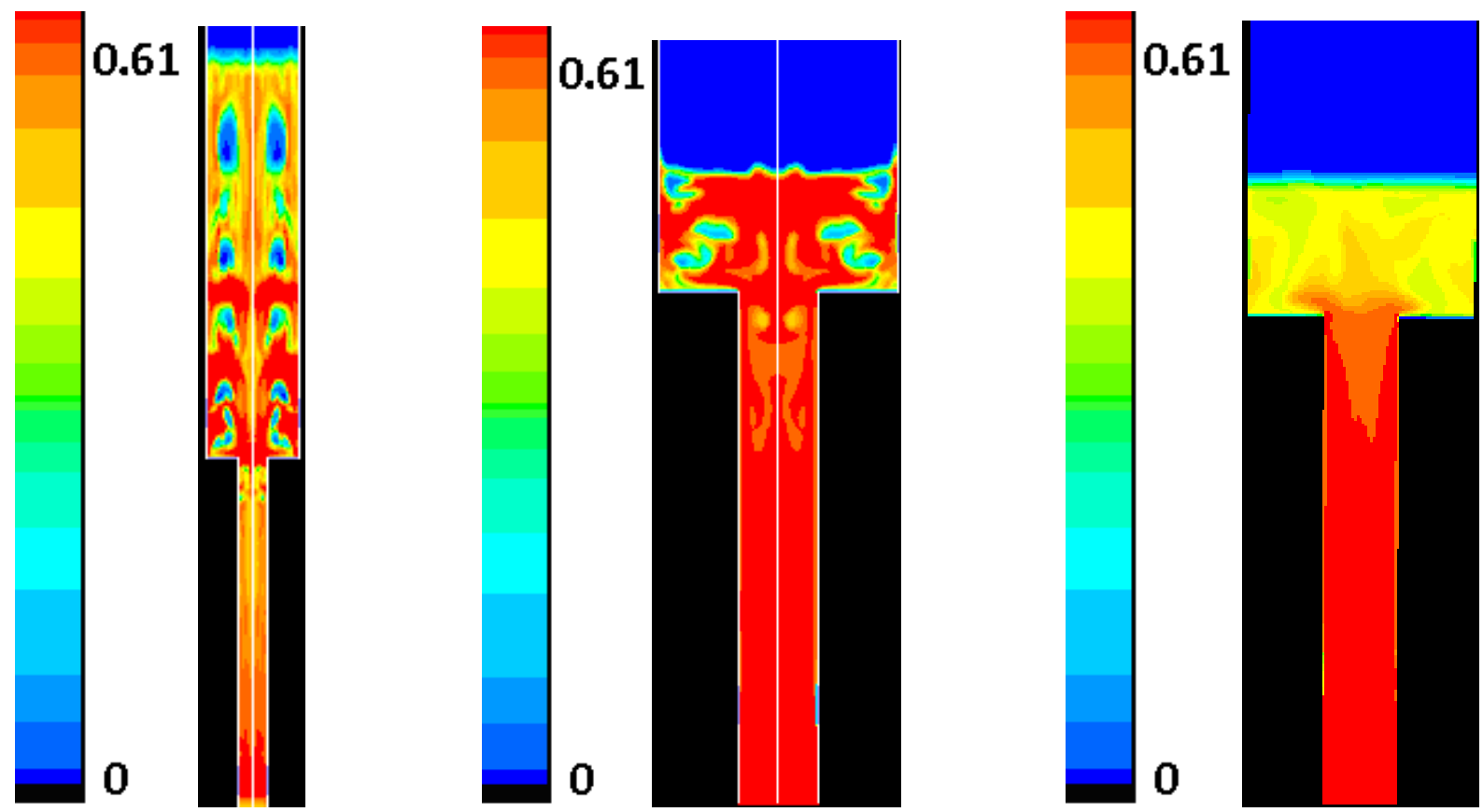

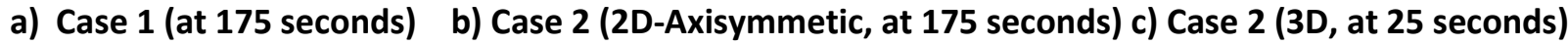
Fig. 10.9: Instantaneous metal oxide volume fraction.
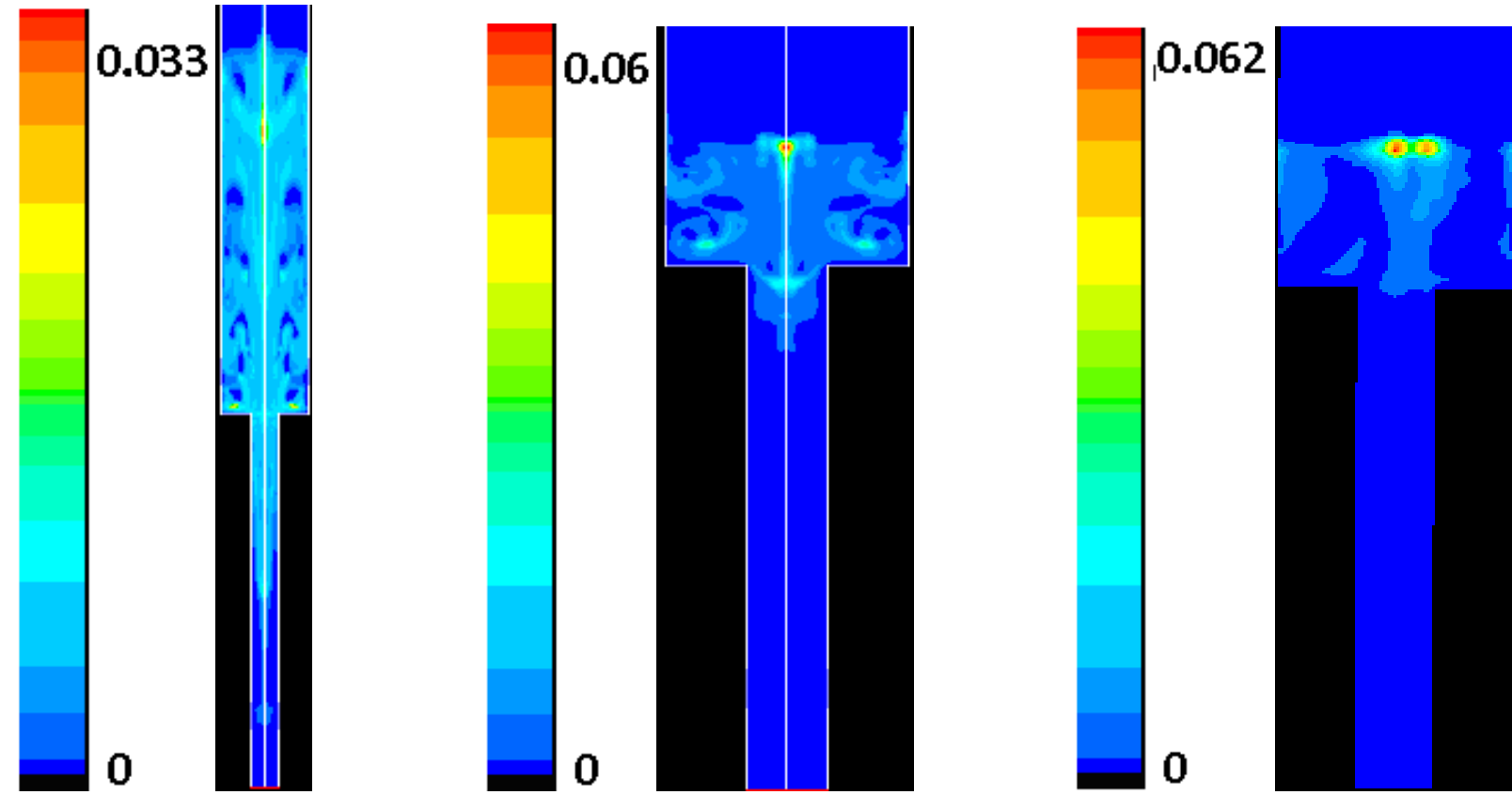

a) Case 1 (at 175 seconds)

b) Case 2 (2D-Axisymmetic, at 175 seconds) c) Case 2 (3D, at 25 seconds) Fig. 10.10: Instantaneous char volume fraction. 
Table 10. 4: Comparison of char leakage and percentage combustion with increase in solid inventory.

\begin{tabular}{|l|r|r|r|r|r|}
\hline $\begin{array}{l}\text { Solid } \\
\text { Inventory }\end{array}$ & Char Leakage & \multicolumn{1}{l|}{$X_{\mathrm{CO}}$} & \multicolumn{1}{l|}{$\mathrm{X}_{\mathrm{H} 2}$} & \multicolumn{1}{l|}{$\mathrm{X}_{\mathrm{CO} 2}$} & \multicolumn{1}{l|}{\begin{tabular}{l} 
Combustion \\
\hline $5 \mathrm{~kg}$
\end{tabular}} \\
\hline $10 \mathrm{~kg}$ & $39.50 \%$ & 0.022 & 0.009 & 0.31 & $90.9 \%$ \\
\hline $21 \mathrm{~kg}$ & $21.86 \%$ & 0.0061 & 0.0029 & 0.32 & $97.26 \%$ \\
\hline
\end{tabular}
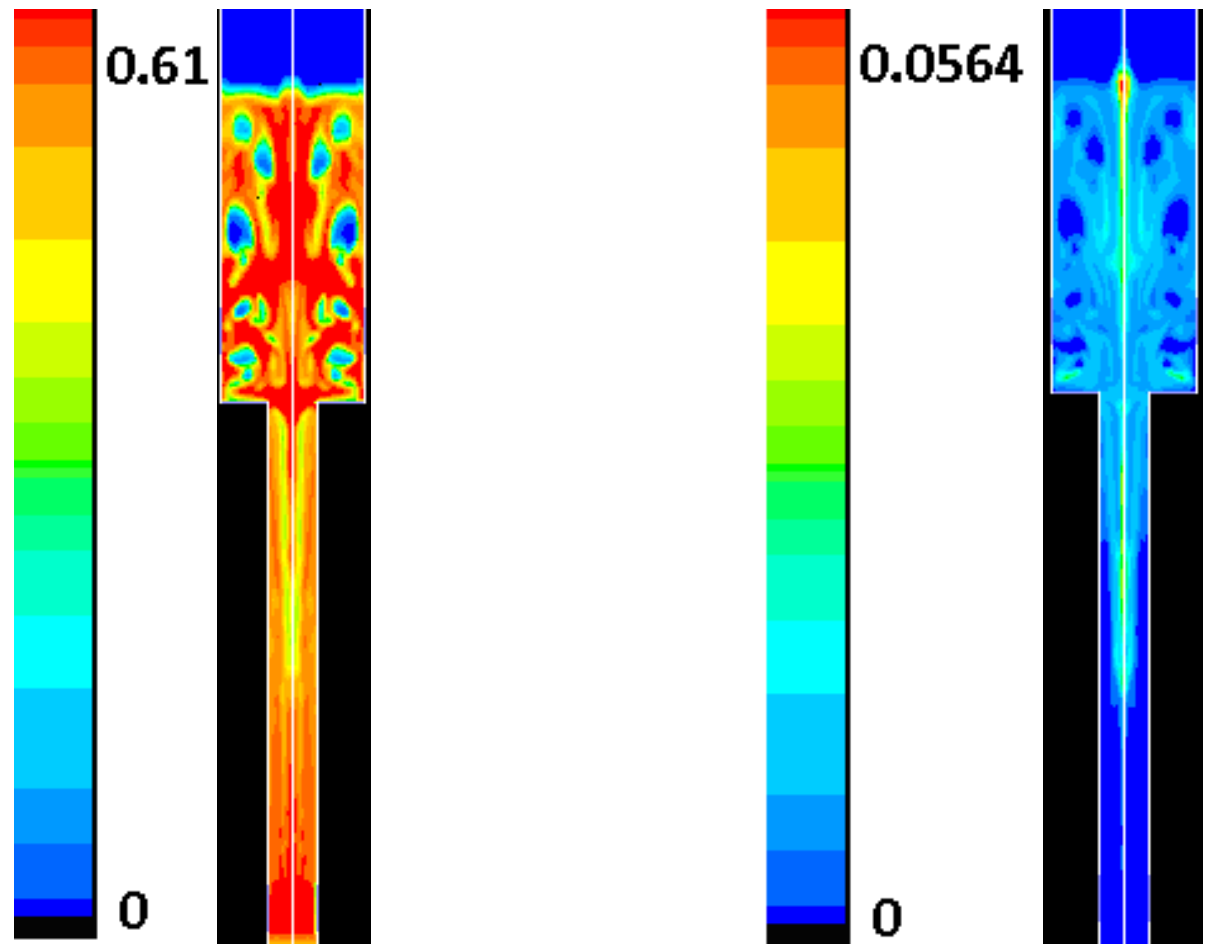
a) $\mathrm{MeO}$
b) Char

Fig. 10.11: Instantaneous volume fraction, Case 1, $10 \mathrm{~kg}$ inventory ( 175 seconds of simulation time). 


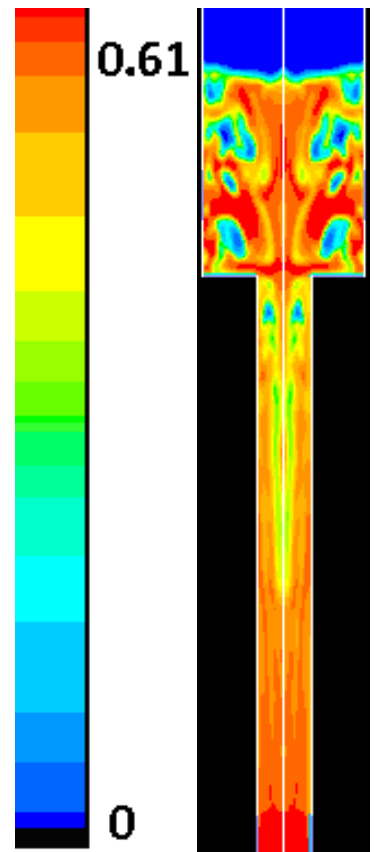

a) $\mathrm{MeO}$

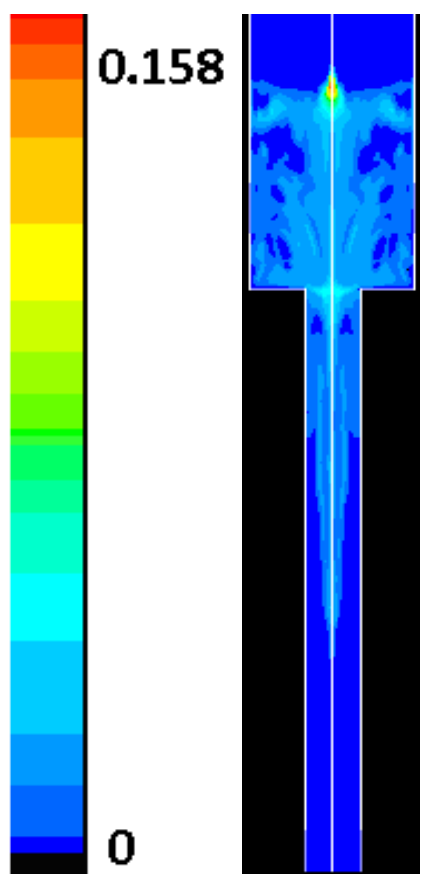

b) Char

Fig. 10.12: Instantaneous volume fraction, Case 1, $5 \mathrm{~kg}$ inventory ( 175 seconds of simulation time).

\section{Effect of Cone Angle}

To analyze the effect of cone angle three different angles of $0^{\circ}, 45^{\circ}$ and $60^{\circ}$ were studied for case 1 with $20 \mathrm{~kg}$ of metal oxides. Fig. 10.13 shows examples of the computed MeO volume fraction for all three cone angles. Bubble formation can be observed in all of the cases. Table 10.5 provides the comparison of the computed char leakage and \% combustion for the three cone angles. No significant differences could be ascertained with changes in the cone angle. Fig. 10.14 shows examples of the char volume fraction. From Figs. $10.14 b$ and $10.14 c$ some tendency for the char particles to accumulate in the cone region of the bed at large cone angles is observed. This is attributed to a recirculation zone created in this region where the lighter char particles congregate (Also see Figs. 10.15b and 10.16b). Fig. 10.15 shows the velocity vectors for the 0 degree cone angle. A strong downward central flow is observed in Fig. $10.15 \mathrm{~b}$ for the solid particles. This is primarily because the central core is the region of least fluidization. The outer annular region has high velocity which causes the particles to move upwards and then later on get entrained into a downward jet in the lower velocity central core of the reactor. The flow is turned radially, as shown by white arrows when it reaches section 1 of the reactor (Figs. 10.15b and 10.16b). Fig. 10.16 shows the velocity vectors for the 60 degree cone angle. A downwards central jet similar to Fig. 10.15 is observed. The cone angle of the reactor helps in creating a recirculation zone inside the cone causing the char to accumulate there (Fig. 10.16b). 

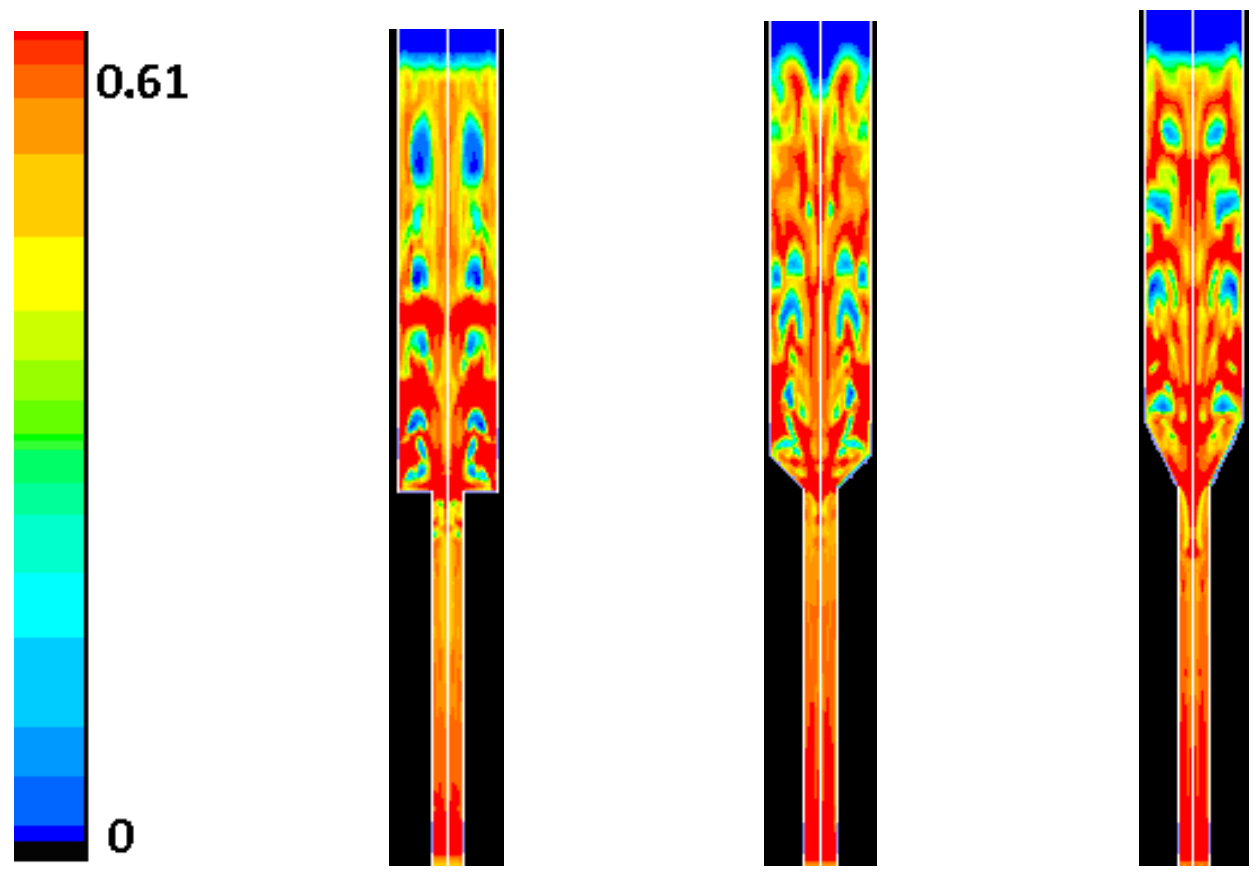

a) $0^{\circ}$

b) $45^{\circ}$

c) $60^{\circ}$

Fig. 10.13: Instantaneous MeO volume fraction, $20 \mathrm{~kg} \mathrm{MeO}$ inventory ( 175 seconds of simulation time).

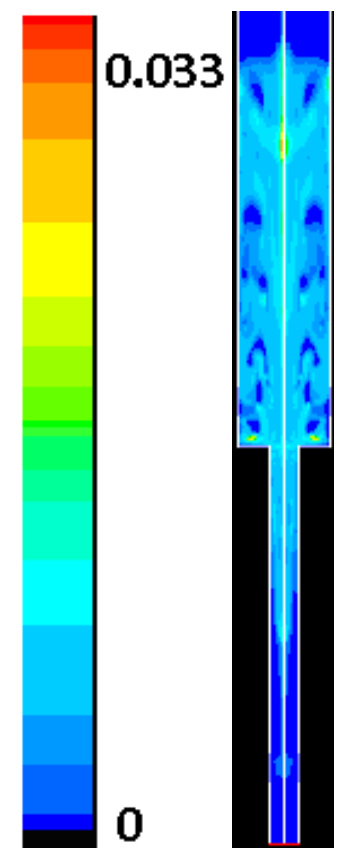

a) $0^{\circ}$

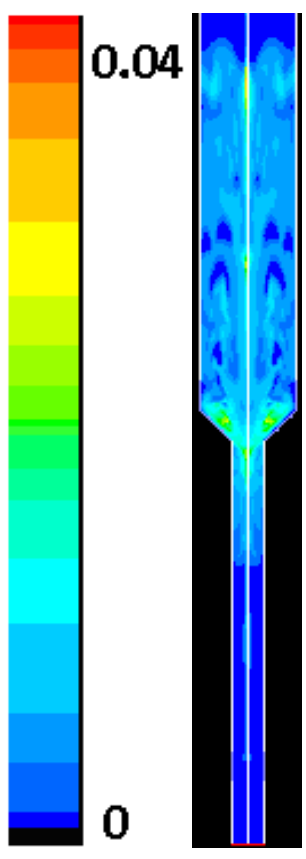

b) $45^{\circ}$

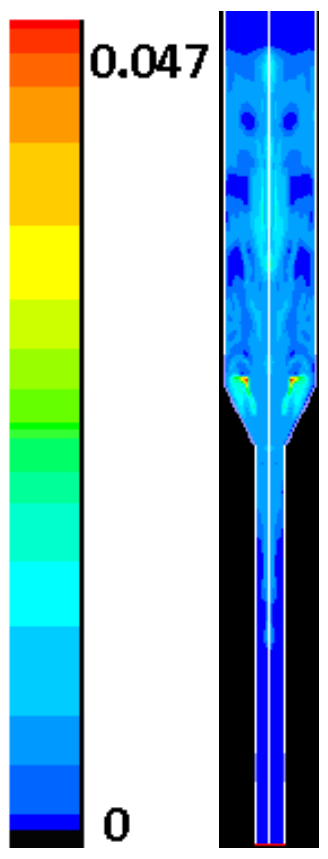

c) $60^{\circ}$

Fig. 10.14: Instantaneous char volume fraction ( 175 seconds of simulation time). 

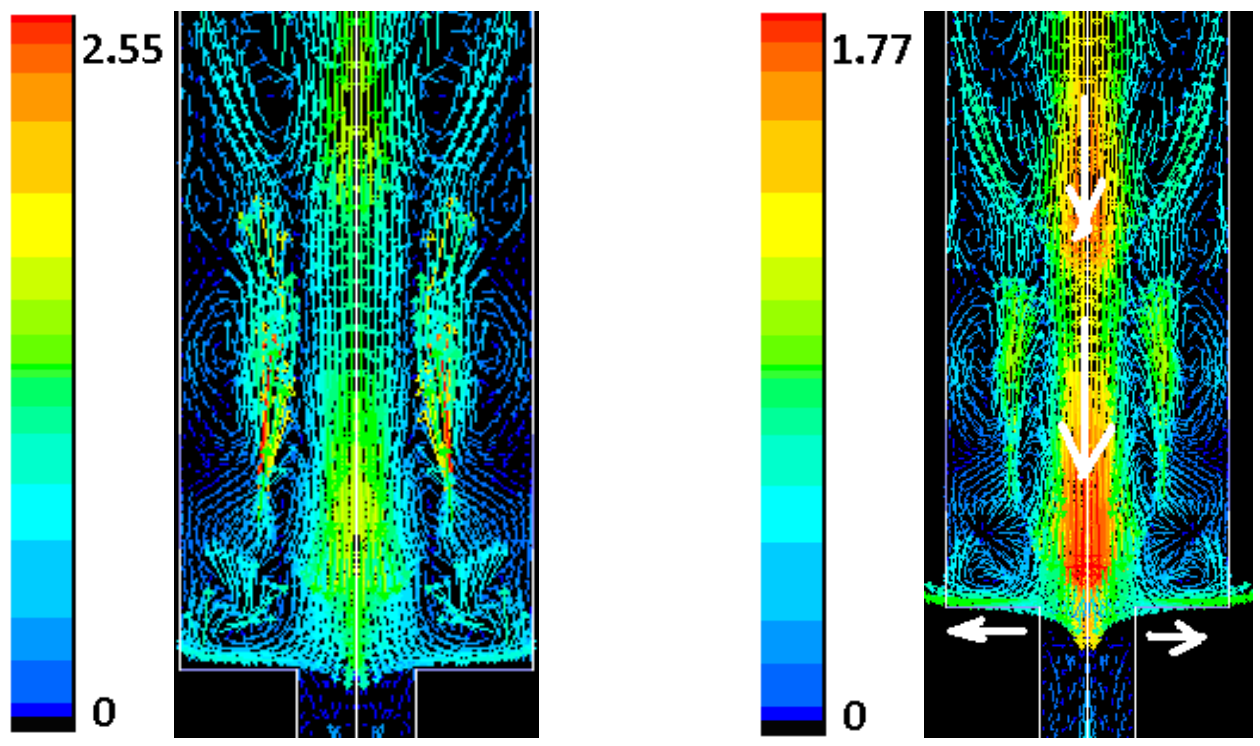

a) Gas velocity vectors

b) MeO velocity vectors (Char velocity is similar) Fig. 10.15: Velocity Vectors for $0^{\circ}$ case ( 175 seconds of simulation time).
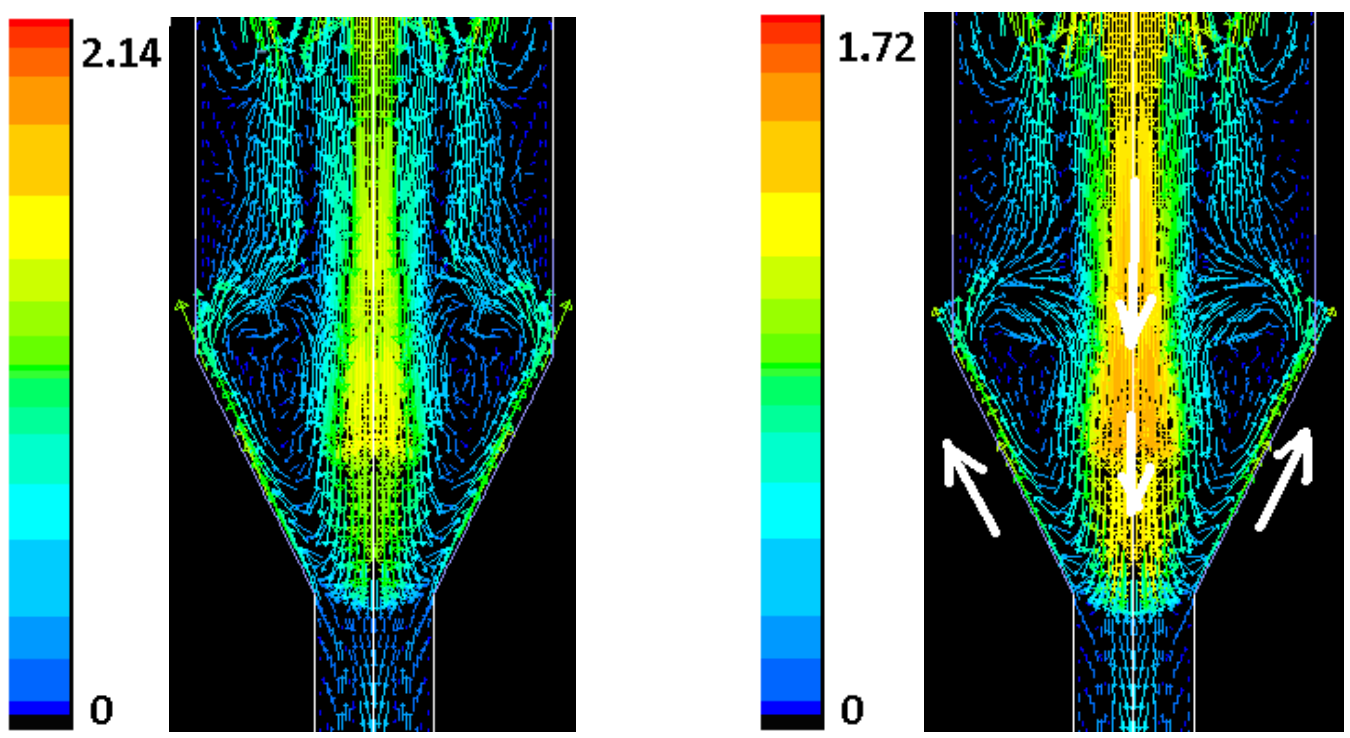

a) Gas velocity vectors

b) $\mathrm{MeO}$ velocity vectors (Char velocity is similar) Fig. 10.16: Velocity Vectors for $60^{\circ}$ case ( 175 seconds of simulation time). 
Table 10.5: Comparison of char leakage and percentage combustion for different cone angles.

\begin{tabular}{|l|r|r|r|r|r|}
\hline Cone Angle & \multicolumn{1}{l|l}{$\begin{array}{l}\text { Char } \\
\text { Leakage }\end{array}$} & $X_{\mathrm{CO}}$ & \multicolumn{1}{l|}{$\mathrm{X}_{\mathrm{H} 2}$} & \multicolumn{1}{l|}{$\mathrm{X}_{\mathrm{CO} 2}$} & $\begin{array}{l}\text { Combustion } \\
\text { Comb }\end{array}$ \\
\hline $0^{\circ}$ & $21.86 \%$ & 0.0061 & 0.0029 & 0.32 & $97.3 \%$ \\
\hline $45^{\circ}$ & $19.35 \%$ & 0.0058 & 0.0025 & 0.32 & $97.4 \%$ \\
\hline $60^{\circ}$ & $20.70 \%$ & 0.0059 & 0.0026 & 0.32 & $97.3 \%$ \\
\hline
\end{tabular}

10.3.3: Details of Design 3:

Design 3 uses a multi-compartment FR to achieve good percentage combustion as well as high carbon capture efficiencies. As shown schematically in Fig. 10.17 the Design 3 FR is divided into four different compartments each of which has a different size and fluidizing velocity.

- Compartment 1: This is the compartment where char is introduced and gasified. This compartment design prevents leakage of char to other compartments. The fluidizing velocity there is equal to the minimum fluidization value for the metal oxide $(0.02 \mathrm{~m} / \mathrm{s})$ which causes the char particles to float to the top. The bubble formation is suppressed which prevents the mixing of light and heavy particles.

- Compartment 2: This compartment is used for pressure balancing that prevents the short-circuit of fluidizing gases from compartment 1 directly to compartment 3. It also helps in controlling the solid circulation rates of the reactor. It is actually a non-mechanical valve. The fluidizing velocity in this compartment is equal to the minimum fluidization velocity of metal oxide particles $(0.02 \mathrm{~m} / \mathrm{s})$.

- Compartment 3/4: The flow in these compartments allow sufficient residence time for the reaction of combustible gases ( $\mathrm{CO}$ and $\mathrm{H}_{2}$ ) with metal oxides.

Fig. 10.18 shows the actual CFD geometry used in the analysis; the sketch in Fig. 10.17 has been stretched horizontally to show the gas, char and metal oxide flow paths more clearly. The dimensions of the actual design are listed in Fig. 10.17. 


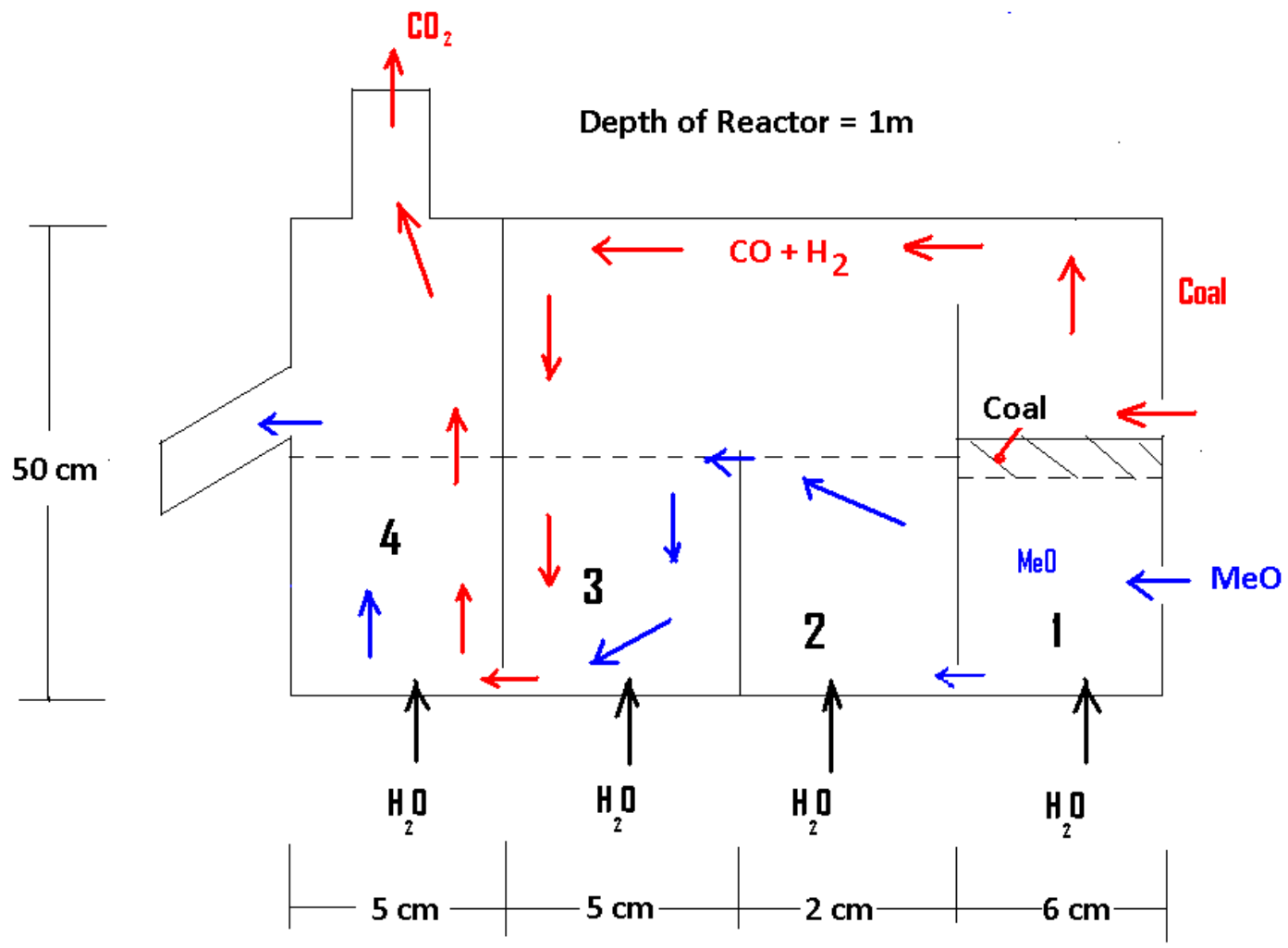

Fig. 10.17: Different compartments in FR for Design 2 (Figure not to scale). The red arrows indicate the flow of char and combustible gases. The blue arrows indicate flow of metal oxide.

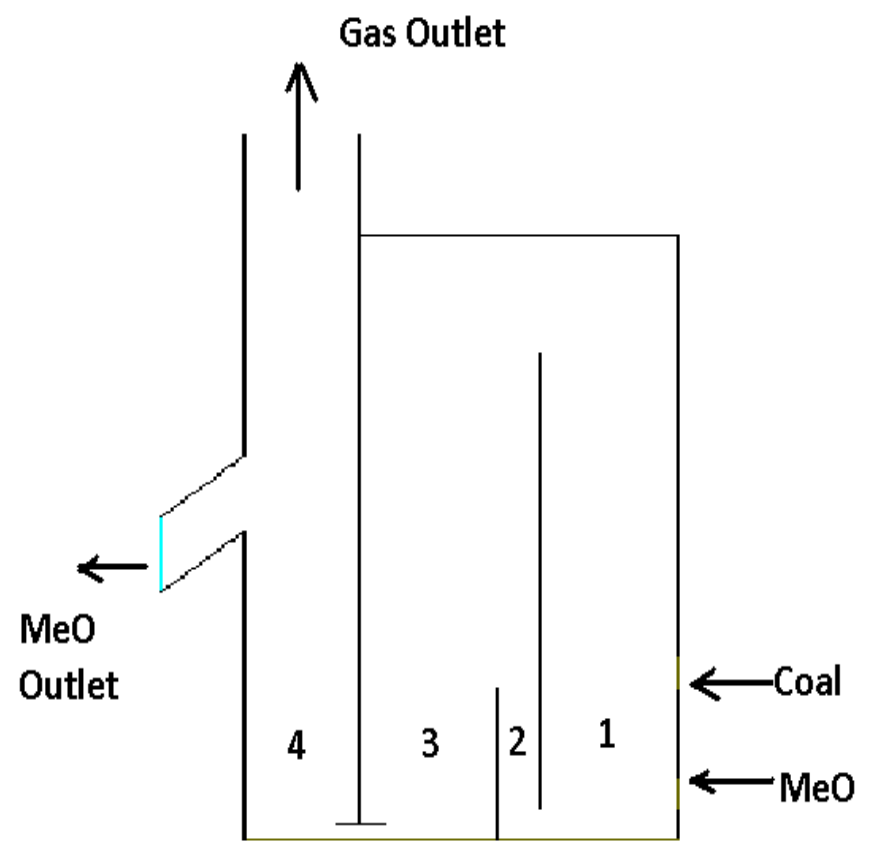

Fig. 10.18: Geometry used in the analysis (shown to scale). 


\subsubsection{Design of the Reactor}

\section{Design of Compartment 1}

The essential goals of this compartment are to segregate the char and $\mathrm{MeO}$ particles as well as provide enough steam to gasify the char at a fast rate. Also the incoming hot metal oxides from the AR have to provide sufficient heat for the gasification process.

The char gasification reaction is given as Equation (10.3).

The stoichiometric mass flow rate of $\mathrm{H}_{2} \mathrm{O}$ that is required is:

$$
\dot{m}_{\mathrm{H}_{2} \mathrm{O} \text { Stoichiomaric }}=M W_{\mathrm{H}_{2} \mathrm{O}} \frac{\dot{m}_{\text {Fuel }}}{M W_{\text {Fuel }}} \frac{v_{\mathrm{H}_{2} \mathrm{O}}}{v_{\text {Carbon }}}=18 \times \frac{0.0000667}{12} \times \frac{1}{1}=0.0001 \mathrm{~kg} / \mathrm{s}
$$

Here $v_{\mathrm{H}_{2} \mathrm{O}}$ and $v_{\text {Carbon }}$ are the stoichiometric coefficients of carbon and steam in the gasification reaction. It is desirable to have extra $\mathrm{H}_{2} \mathrm{O}$ concentrations to effectively gasify the char. Therefore $100 \%$ higher steam mass flow rates have been used:

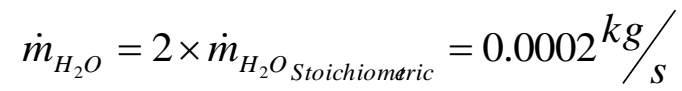

To get segregation of char and $\mathrm{MeO}$ a velocity which is close to the minimum fluidization velocity of the $\mathrm{MeO}$ has been selected. Fluidizing at close to the $U_{m f}$ prevents bubble formation that is primarily responsible for mixing of the different solid species. The velocity of steam in compartment 1 is therefore selected to be $0.02 \mathrm{~m} / \mathrm{s}$. Steam is used as the fluidizing gas in this section.

The area of compartment 1 is calculated from mass flow rate of steam as:

$$
\text { Area }_{1}=\frac{\dot{m}_{\text {Steam }}}{\rho_{\text {steam }} u_{\text {steam }}}=\frac{0.0002}{0.17 \times 0.02}=0.058 \mathrm{~m}^{2} \sim 0.06 \mathrm{~m}^{2}
$$

The depth of the bed (distance perpendicular to plane/paper) is assumed to be $1 \mathrm{~m}$ (Fig. 10.17).

The width of the compartment 1 is then $0.06 \mathrm{~m}$ or $6 \mathrm{~cm}$ so as to provide the required flow cross sectional area. 


\section{Design of Compartment 2}

The purpose of compartment 2 is primarily to control the solid flow rate and to prevent shortcircuiting of fluidizing gases from compartment 1 directly to compartment 3 . The width of compartment 2 is not particularly very important, though it should be wide enough to allow for smooth transport of solid particles. An arbitrary width of $2 \mathrm{~cm}$ has been selected for this compartment. Also it is important that this compartment is fluidized to allow easy transport of particles. The fluidizing velocity in compartment 2 is close to minimum fluidization of the metal oxide particles $(0.02 \mathrm{~m} / \mathrm{s})$. Steam is used as the fluidizing gas in this section.

\section{Design of Compartment 3 and 4}

The combustion of combustible gases such as $\mathrm{CO}$ and $\mathrm{H}_{2}$ takes place in compartments 3 and 4 . It is therefore desirable that these compartments have sufficient metal oxide mass to allow for complete combustion. The two main combustible gases produced are $\mathrm{CO}$ and $\mathrm{H}_{2}$. CO reaction rates are slower than $\mathrm{H}_{2}$ and therefore ensuring complete $\mathrm{CO}$ consumption is more important. The mass flow rate of carbon is $0.0000667 \mathrm{~kg} / \mathrm{s}$. The carbon monoxide flow rate is then given as:

$$
\dot{m}_{C O}=M W_{C O} \frac{\dot{m}_{\text {Fuel }}}{M W_{\text {Fuel }}}=28 \times \frac{0.0000667}{12}=0.000156 \mathrm{~kg} / \mathrm{s}
$$

Based on $\mathrm{CO}$ and metal oxide reaction rates provided in Chapter 7, it is possible to calculate the average rate of consumption of $\mathrm{CO}$ at a metal oxide volume fraction of 0.6 and at a $\mathrm{CO}$ concentration of $1 \mathrm{e}-4 \mathrm{kmol} / \mathrm{m}^{3}$ (The concentrations provided are typical values expected in the bed). The average reaction rate has been estimated to be:

Average Rate of Consumption $C O\left(\frac{\mathrm{kg}}{\mathrm{m}^{3} \mathrm{~s}}\right)=0.13 \frac{\mathrm{kg}}{\mathrm{m}^{3} \mathrm{~s}}$

The volume of metal oxide required to completely consume the $\mathrm{CO}$ is then given by: Volume of $\mathrm{MeO}=\frac{\dot{m}_{C O}}{\text { Average_Rate }}=\frac{0.000156}{0.13}=0.0012 \mathrm{~m}^{3}$

Then, a minimum inventory of $4.2 \mathrm{~kg}$ of metal oxide mass is required assuming good contact between metal oxide and reacting gases take place. 
It is desirable to have several multiples of this volume of metal oxide to ensure that complete combustion can be achieved. A factor of 10 times the minimum required metal oxide volume has been used in the present case. Assuming a bed height of $10 \mathrm{~cm}$ in the two compartments the width of the compartments are estimated to be $5 \mathrm{~cm}$ (Depth into the page has been previously assumed to be $1 \mathrm{~m}$ ).

\section{Details of the CFD Analysis:}

The purpose of the present analysis has been to qualitatively check the suitability of the design and verify the concepts. A mesh of about 11000 cells was used in the analysis. The quadratic upwind interpolation scheme (QUICK) has been used for the volume fraction equations and second order upwind discretization schemes were used for solution of all other equations, while first order implicit temporal discretization was used.

Fig. 10.19 shows the example of the computed volume fractions of char and metal oxide at 50 seconds of simulation time. The char is clearly observed to be floating on the top of the bed in compartment 1 . The fluidizing velocities in compartments 1 and 2 are just above the minimum fluidization velocity of the heavier metal oxide particles $(0.02 \mathrm{~m} / \mathrm{s})$. The low fluidizing velocity suppresses any bubble formation which would result in mixing of the char and metal oxide. In the absence of bubbles, the char particles float to the top. Also some overflow of metal oxide from compartment 2 to compartment 3 can be observed in Fig. 10.19a. Since there is a constant stream of incoming metal oxide, the periodic overflow of metal oxides has to be expected.

Fig. 10.20a shows the computed mole fraction of $\mathrm{CO}$ at the same time instant. The CO formation primarily occurs in the char layer floating at the top of the bed in compartment 1 . The CO then gets transported to compartments 3 and 4 due to the pressure gradients in the system (Fig. 10.21b). In compartments 3 and 4 the CO reacts with the metal oxide and gets completely consumed. The Design 1 and Design 2 described in the previous section of this chapter had incomplete combustion as the combustible gases released by char floating on top of the bed could not completely react with the metal oxide. In the present case (Design 3 ) this is avoided as the char is segregated and gasified in compartment 1. Fig. $10.20 \mathrm{~b}$ shows the mole fraction of $\mathrm{CO}_{2}$. High $\mathrm{CO}_{2}$ concentration is found in compartments 3 and 4 due to the reaction between $\mathrm{CO}$ and the metal oxide. Fig. 10.20c shows the $\mathrm{H}_{2} \mathrm{O}$ mole fraction. High concentrations can be found in the inlets of compartment 1 and 2 which are fluidized with steam. There is practically no $\mathrm{CO}$ or $\mathrm{CO}_{2}$ leaking into the compartment 4 . This is essential for high $\mathrm{CO}_{2}$ capture efficiency. Table 10.6 shows the percentage combustion and char leakage 144 
observed. The percentage combustion is $100 \%$ based on stated assumptions. Also the isolation of char in chamber 1 prevents any leakage to the AR resulting in complete carbon capture.

Fig. 10.21a shows the computed temperature contours. Low temperatures are found in compartment 1 where the endothermic gasification reaction occurs. Fig. 10.21b shows the pressure contour plot. High pressures exist in compartment 1 and this pressure gradient is primarily responsible for the transport of gases in the reactor.

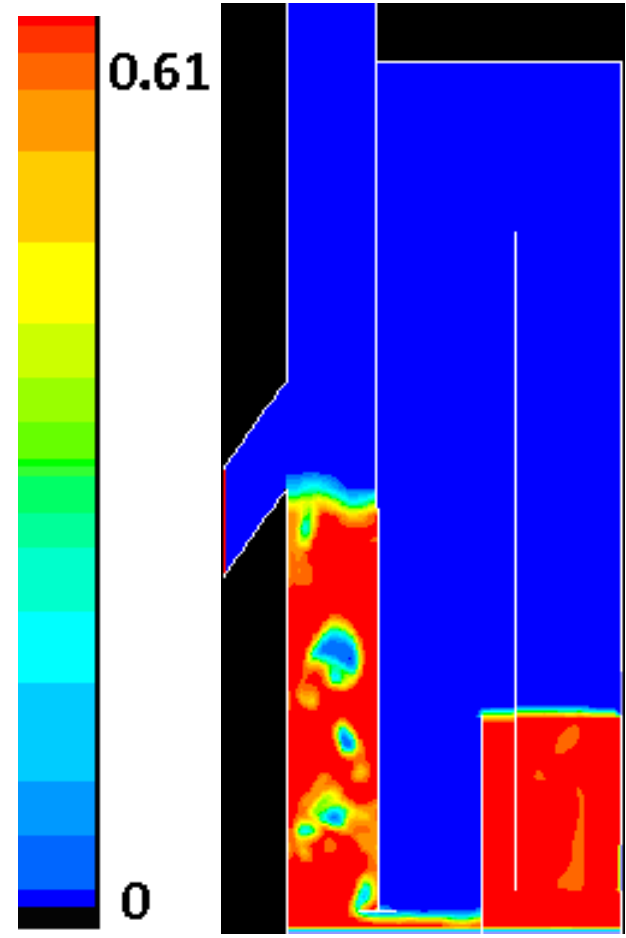

a) $\mathrm{MeO}$

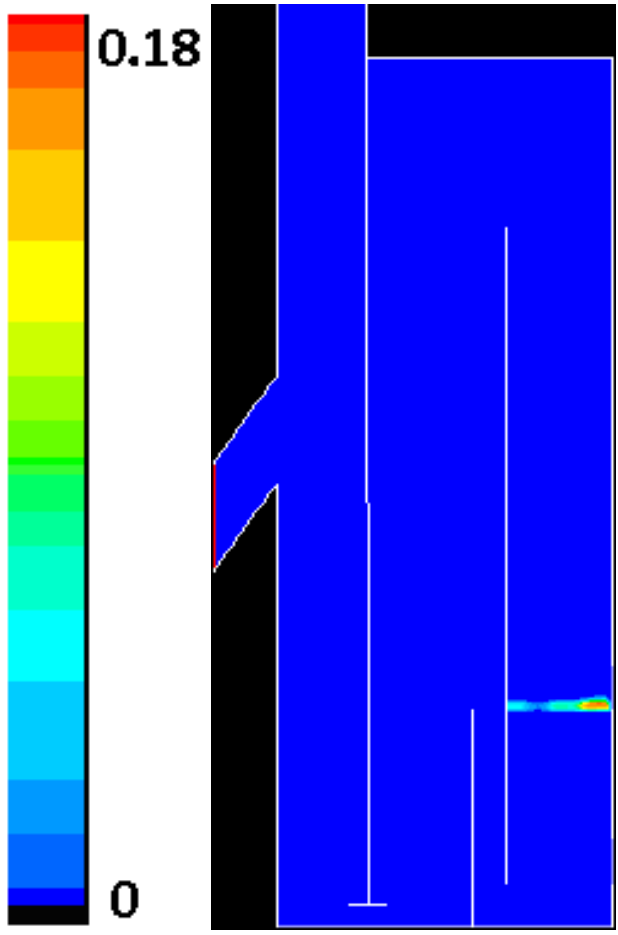

b) Carbon

Fig. 10.19: Volume fraction and bed heights at $\sim 95$ seconds of simulation time.

Table 10.6: Char leakage and percentage combustion for Design 3.

\begin{tabular}{|l|l|l|l|l|l|}
\hline & $\begin{array}{l}\text { Char } \\
\text { Leakage }\end{array}$ & $X_{\mathrm{CO}}$ & $X_{\mathrm{H} 2}$ & $X_{\mathrm{CO} 2}$ & $\begin{array}{l}\% \\
\text { Combustion }\end{array}$ \\
\hline Design 3 & $0 \%$ & 0.0 & 0.00 & 0.14 & $100.0 \%$ \\
\hline
\end{tabular}



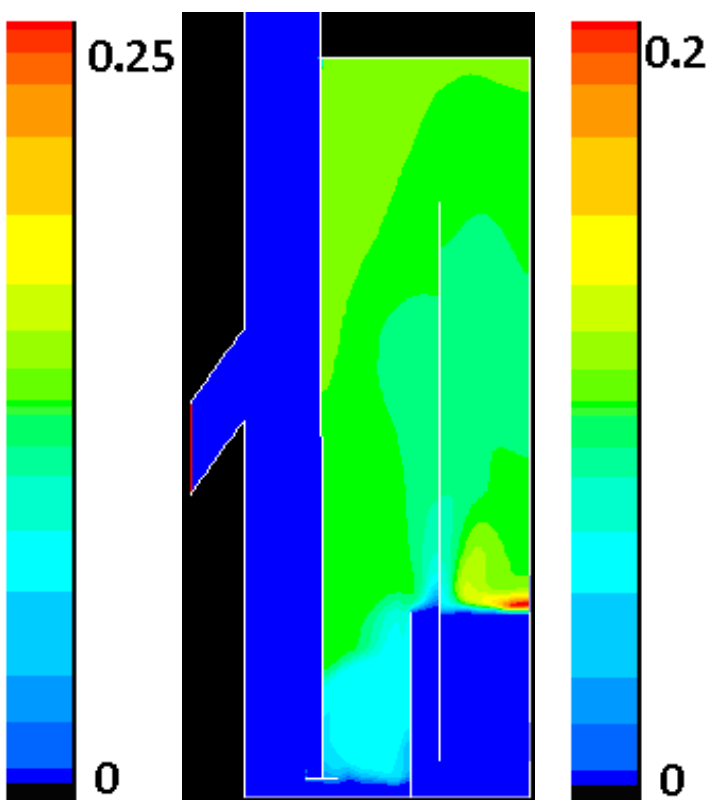

b) $\mathrm{CO}$
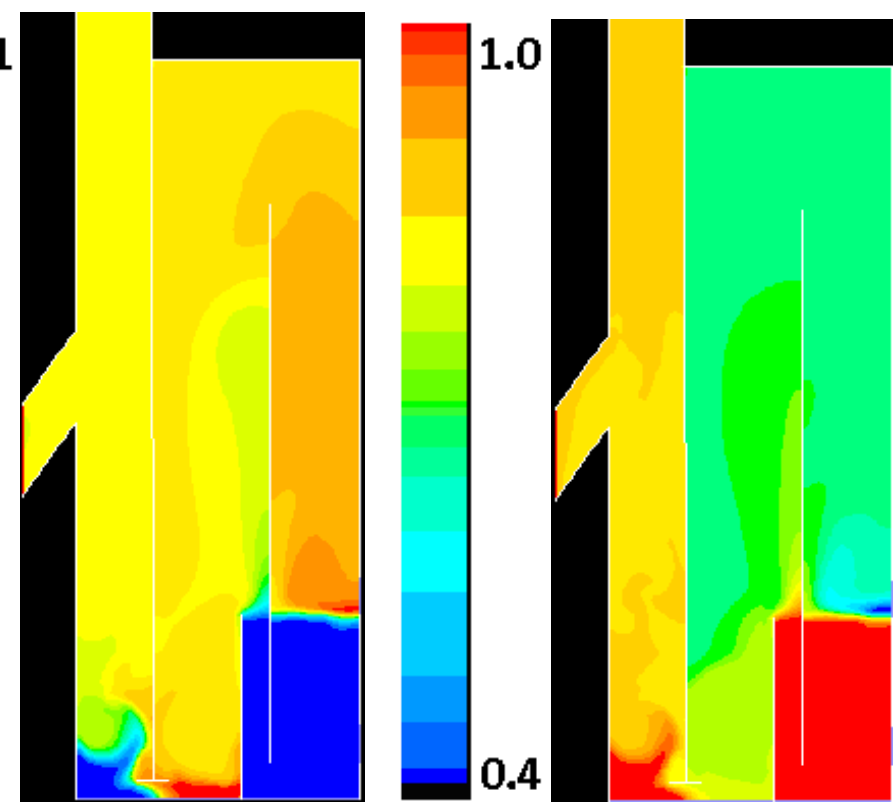

b) $\mathrm{CO}_{2}$

c) $\mathrm{H}_{2} \mathrm{O}$

Fig. 10.20: Mole fraction of flue gases at $~ 95$ seconds.

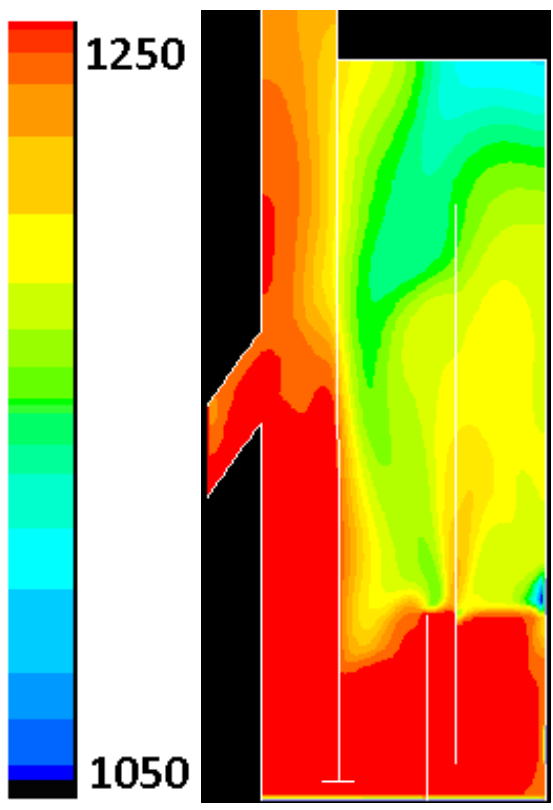

a) Temperature (K)

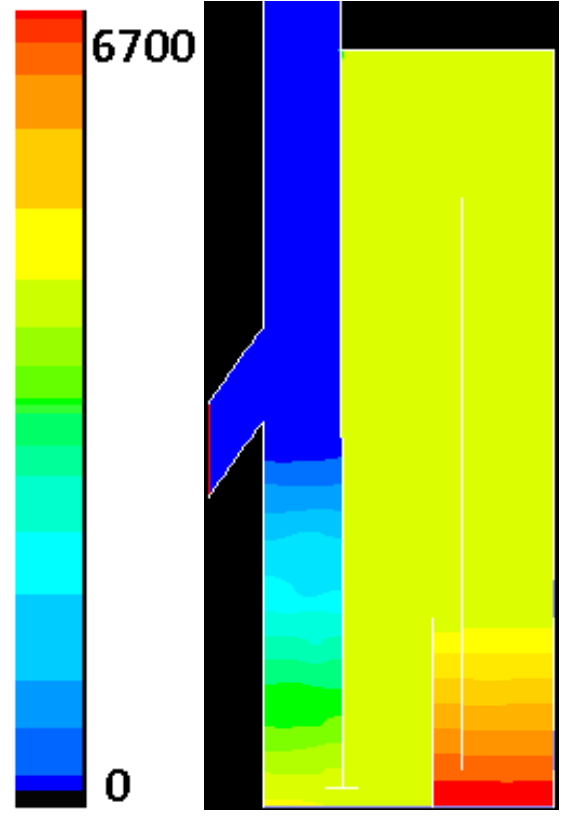

b) Gas pressure (Pascal)

Fig. 10.21: The gas temperature and gas pressure at $\sim 95$ seconds.

\section{4: Comparison of Designs 1,2 and 3 and other experimental studies:}

The designs are compared based on the following parameters.

1) Percentage combustion of the FR. 
2) Carbon capture efficiency.

3) Amount of solid inventory required.

4) Size or space (cross-sectional area) required for the FR.

5) Ease of construction and operation.

Table 10.7 summarizes the comparisons between the various designs. All three designs are capable of providing high percentage combustion (>90\%) (Also see Tables 10.2 and 10.3). The Design 3 was found to provide complete combustion based on the stated modeling assumptions. Table 10.1 shows the various existing studies on CLC reactors utilizing solid fuels. The percentage combustion in these published experimental reactors is usually less than $90 \%$ (Table 10.1). Shen et al. (2009) shows the maximum percentage combustion at $91 \%$, however $\mathrm{H}_{2}$ leakage has been ignored in this calculation. Design 2 and Design 3 therefore show considerable promise in achieving better percentage combustion performance in comparison with existing experimental setups.

Due to the high leakage the carbon capture efficiency of Design 1 was much less than the USDOE specified 90\%. Design 2 was found to be capable of matching this requirement if the diameter of section 1 of the design was large enough to prevent large metal oxide velocities. The Design 3 segregated the char particles in compartment 1 and hence provided $100 \%$ carbon capture based on the stated assumptions. Thus both Design 2 and 3 show promise in achieving carbon capture in excess of $90 \%$. In comparison existing systems show carbon capture that is usually around $80 \%$ or less (Table 10.1).

The amount of solid inventory used in Design 1 was around 20kg. In Design 2 it was between 5 to $28 \mathrm{~kg}$ based on the case considered. The Design 3 however used significantly larger amount of metal oxide inventory at around $56 \mathrm{~kg}$. However it is likely that further optimization of design 3 could lead to significantly less usage of metal oxides.

The cross sectional area occupied by Design 1 was the minimum at $225 \mathrm{~cm}^{2}$. The cross sectional area used by Design 2 varied between $225 \mathrm{~cm}^{2}$ to $900 \mathrm{~cm}^{2}$. For Design 3 the cross sectional area was $2000 \mathrm{~cm}^{2}$. However it is possible that this value could also be significantly reduced on basis of optimization studies of the reactor. 
The ease of construction of Design 1 is the simplest because of its simple one cylinder body. The Design 2 also has a simple cylindrical body that is easy to construct. The Design 3 has multiple compartments which make it more difficult to fabricate and operate as a greater number of variables and parameters have to be controlled. Among the published experimental data on CLC reactors (Table 10.1), the reactor design by Shen et al. (2009) provides a reasonable percentage combustion ( 91\%) and moderate carbon capture ( 78\%). In comparison the reactor Design 2 and 3 show percentage combustion higher than $95 \%$ and carbon capture efficiency greater than $90 \%$.

Table 10.7: Comparison of char leakage and percentage combustion for case 1 and case 2.

\begin{tabular}{|l|l|l|l|}
\hline & Design 1 & Design 2 & Design 3 \\
\hline Combustion efficiency of the FR. & High & High & High \\
\hline Carbon capture efficiency. & Low & Medium & High \\
\hline Amount of solid inventory. & Medium & Medium & $\begin{array}{l}\text { High (The design could be } \\
\text { optimized to use lower } \\
\text { amounts) }\end{array}$ \\
\hline $\begin{array}{l}\text { Size or space (cross sectional area) required } \\
\text { for the FR. }\end{array}$ & Small & Medium & $\begin{array}{l}\text { High (could be optimized to use } \\
\text { less area) }\end{array}$ \\
\hline Ease of construction and operation & Easy & Easy & Difficult \\
\hline
\end{tabular}

\section{5: Conclusion}

A review of the published experimental data on CLC systems utilizing solid fuel shows that the observed percentage combustion is typically low $(<90 \%)$ and the carbon capture efficiencies are also generally below the desired $90 \%$ as prescribed by the United States Department of Energy (USDOE). Three designs have been suggested and analyzed in this chapter. The Design 1 was simple cylindrical reactor where good mixing between the metal oxide and solid fuel is achieved. The CFD simulations of this design indicated a high combustion efficiency of close to $98 \%$ based on the stated assumptions. However the char leakage was also high (>60\%) which is undesirable. The Design 2 was a two section reactor where the section 1 segregated the char from the metal oxide and thus minimized the leakage of char particles to the AR. High combustion efficiency greater than $90 \%$ can also be achieved using this design. The Design 3 was a multi-compartment reactor where the char was segregated and gasified. 
Both the predicted percentage combustion and the carbon capture efficiency for Design 3 were 100\% based on the stated modeling assumptions. The cross-sectional area and the solid inventory used in the Design 3 are both higher than that in Design 1 and 2. However it is expected that further optimization studies can bring these values down considerably. 


\section{Chapter 11: Conclusions and Future Work}

Detailed computational fluid dynamic (CFD) studies of chemical looping combustion systems have been carried out in this research work using the commercial ANSYS-FLUENT ${ }^{\mathrm{TM}}$ CFD solver. An important goal of the research work was to carry out validation studies that access the capability of existing CFD models to predict the complex behavior of CLC reactors. There have been extensive experimental studies in CLC; however existing CFD simulations of this concept are quite limited. Also, no published CFD simulations of CLC systems utilizing solid fuels exist to date in the literature. Heterogeneous reactions have been used to describe the coal conversion and the reactions of the gaseous fuel with the carrier. Global chemical reaction models of fuel and carrier were utilized.

Capability of the model to simulate the segregation processes, depending on particle density and size differences has been tested (Chapter 5 ) and compared with experimental data. It was observed that the CFD model captured the trends in the segregation of particles at low velocity close to minimum fluidization (Goldschmidt et al., 2003). Also, the segregation at high velocity which was several multiples of minimum fluidization (Moritomi et al., 1983) could also be predicted qualitatively by the CFD model. The ability to predict segregation between the carrier and the fuel is critical in designing a reactor with the desired behavior.

The FR is one of the most critical parts of the CLC system because of the need to get high combustion efficiency as well as prevent leakage of fuel or $\mathrm{CO}_{2}$ from the FR to AR. Simulations of the fuel reactors utilizing gaseous fuel described in two experimental studies (Son and Kim, 2006 and Mattisson et al., 2001) have been carried out (Chapter 7). For the Son and Kim (2006) experiments, the CFD model provided reasonable predictions about reactor performance at different fluidizing velocity, metal oxide type, reactor temperature, etc. Also, bubble formation was observed in the reactor, and these bubbles were found to be regions of high fuel concentration. This could possibly be responsible for some part of the un-burnt fuel at the exit. The CFD model also gave a good comparison with the experiments of Mattisson et al. (2001). The numerically predicted time-varying concentration of flue gases matched well with experimental values, when the transformation of $\mathrm{Fe}_{3} \mathrm{O}_{4}$ to $\mathrm{FeO}$ was considered.

The CFD model was also used for simulating a FR utilizing solid fuels (Leion et al., 2008; Chapter 8). Chemical kinetic models were assembled from the literature for the reactions between the 
iron oxide carrier and fuel gases as well as for the devolatilization and char gasification of coal. The model was able to predict the transient outlet concentrations of $\mathrm{CO}_{2}, \mathrm{CO}$ and $\mathrm{CH}_{4}$. The reactor performance at different operating temperatures and inlet steam concentrations was captured in a reasonable manner. This demonstrates that CFD modeling can be an effective approach in the design of such a reactor.

The design of a chemical looping combustion system requires that a number of important parameters be considered, including:

- Combustion efficiency of the reactor,

- Carbon capture efficiency of the reactor,

- Mass distribution of metal oxides in the air and fuel reactors,

- Solid circulation rate to provide enough oxygen for combustion of fuel and heat balance between reactors, and

- Gas leakage between reactors which can result in lower carbon capture efficiency and dilution of $\mathrm{CO}_{2}$ concentration in the $\mathrm{FR}$ exhaust gases.

The use of CFD can help in estimating the performance of the reactor based on the above parameters without making detailed assumptions in the modeling procedure. A CFD simulation involving a complete chemical looping combustion system for which detailed experimental data are available has been carried out as described in Chapter 9 (Abad et al., 2006). The model was able to predict the outlet concentrations of $\mathrm{CO}_{2}$, and $\mathrm{CH}_{4}$. The reactor performance at different operating temperatures was captured in a reasonable manner. It was found that the circulation of solids between the reactors is controlled by the frictional flow in the downcomer of the reactor and hence predicting the correct amount of frictional energy dissipation was critical in obtaining accurate results. The performance of the reactor at different temperatures was also predicted accurately. The predicted gas leakage and dilution was within the observed variance reported by Abad et al. (2006). The solid mass distribution in the AR and FR was predicted accurately.

A review of the limited published experimental data on CLC systems utilizing solid fuel shows that the percentage combustion that is obtained is typically low $(<90 \%)$ and the carbon capture efficiencies are also below the desired $90 \%$ as prescribed by the United States Department of Energy (USDOE). Three FR designs have been suggested to achieve high percentage combustion and carbon 
capture. These designs have been analyzed with CFD. It was found that high carbon capture efficiency as well as percentage combustion can be achieved with two of these design concepts.

Future work: A considerable amount of work still needs to be done to optimize the designs that have been described in the present study. Many of the experimental studies in the literature on solid fuel combustion with CLC report combustion efficiencies and carbon capture efficiencies that are well below $100 \%$. CFD will be very useful as a tool to optimize the designs reported in the present study as well as develop new ones. A complete CLC system utilizing solid fuels has not been simulated in the present work; this should be attempted in the future. Also, additional validation studies should be performed for other MeO carrier materials. 


\section{Nomenclature}

a Pre-exponential factor

$A_{p} \quad$ Projected area of the particle

[A] Molar concentration of species A and similarly for other species

AR Air reactor

b Stoichiometric coefficient of solid species ' $B$ '

$c^{\prime} \quad$ Random fluctuating part of the particle velocity

c Velocity of particle

$C_{A} \quad$ Concentration of reactant A

$C_{C} \quad$ Concentration of product C

$C_{D} \quad$ Drag coefficient.

$C_{o x} \quad$ Oxygen carrying capacity of the metal oxide

$C_{i} \quad$ Concentration of reactant ' $\mathrm{i}$ '

$C_{p} \quad$ Specific heat

Cell _ Vol Volume of computational cell in CFD analysis

D Dilution due to gas leakage in CLC reactors

$D_{e} \quad$ Diffusion coefficient

$d_{p} \quad$ Diameter of particle

$d_{s} \quad$ Diameter of particle of solid phase

E Activation energy

$E_{s} \quad$ Kinetic energy of the random motion 
$e_{s s} \quad$ Coefficient of restitution

$\mathrm{f} \quad$ Distribution function in single-particle phase space

F $\quad$ Force field acting on particles

$\mathrm{Fr} \quad$ Coefficient in equation for frictional pressure

FR Fuel reactor

$g \quad$ Acceleration due to gravity

$g_{0, s s} \quad$ Radial distribution function

$H V_{i} \quad$ Heating value of the fuel

$\dot{h}_{f} \quad$ Enthalpy of formation

$<\mathrm{h}>\quad$ Average bed height

$\overline{\bar{I}} \quad$ Identity stress tensor

$I_{2 D} \quad$ Second invariant of strain tensor

k Preexponential factor or rate constant for a given reaction

$k_{b} \quad$ Backward rate constant

$k_{f} \quad$ Forward rate constant

$k_{g} \quad$ Diffusion coefficient

$k_{\mathrm{H}_{2} \mathrm{O}} \quad$ Coefficient in gasification reaction rate

$k_{V} \quad$ Devolatilization rate constant

$K_{E} \quad$ Equilibrium constant to account for the backward rate

$L \quad$ Leakage of gas from $F R$ to $A R$

m Mass of particle

$m_{o x} \quad$ Mass in particle in fully oxidized (ox) form

$m_{\text {red }} \quad$ Mass in particle in fully reduced (red) form 
$\dot{m}_{\text {air }} \quad$ Mass flow rate of air

$\dot{m}_{\mathrm{CO} 2}$ Mass flow rate of $\mathrm{CO}_{2}$

$\dot{m}_{f} \quad$ Mass flow rate of fuel

$\dot{m}_{\mathrm{H} 2 \mathrm{O}}$ Mass flow rate of $\mathrm{H}_{2} \mathrm{O}$

$\dot{m}_{i} \quad$ Mass flow rate of the $\mathrm{i}^{\text {th }}$ species

$\dot{m}_{i j}^{q p} \quad$ Mass transfer rate from the $j^{\text {th }}$ species of the $p^{\text {th }}$ phase to $i^{\text {th }}$ species contained in the $q^{\text {th }}$ phase

$\dot{m}_{N 2} \quad$ Mass flow rate of Nitrogen

$\dot{m}_{O_{2}} \quad$ Mass flow rate of oxygen

$\dot{m}_{o x_{-} i n}$ Mass flow rate of solid metal oxide particles which are in oxidized form

$\dot{m}_{\text {red_in }}$ Mass flow rate of solid metal oxide particles which are in reduced form

$\dot{m}_{p q} \quad$ Mass transfer from the $\mathrm{p}^{\text {th }}$ phase to $\mathrm{q}^{\text {th }}$ phase

$\dot{m}_{r s} \quad$ Mass transfer from the $r^{\text {th }}$ phase to $s^{\text {th }}$ phase

$\dot{m}_{\text {Solids }}$ Circulation rate of solid metal oxide particles between FR and AR

$\dot{m}_{\text {solid_in }}$ Mass flow of solid metal oxide particles into the reactor

$\dot{m}_{\text {solid_out }}$ Mass flow of solid metal oxide particles out of the reactor

$\dot{m}_{s g} \quad$ Mass transfer rate $(\mathrm{kg} / \mathrm{m3} / \mathrm{s})$ between gas phase and the $\mathrm{s}^{\text {th }}$ solid phase

$M_{o x} \quad$ Mass per unit volume of metal oxide in fully oxidized (ox) form due to presence of ' $n$ ' particles

$M_{\text {red }} \quad$ Mass per unit volume of metal oxide in fully reduced (red) form due to presence of ' $n$ ' particles

$M W_{i} \quad$ Molecular weight of the $\mathrm{i}^{\text {th }}$ species

$M W_{\mathrm{CO} 2}$ Molecular weight of $\mathrm{CO}_{2}$

$M W_{\text {Fuel }}$ Molecular weight of Fuel

$M W_{\mathrm{H}_{2} \mathrm{O}}$ Molecular weight of $\mathrm{H}_{2} \mathrm{O}$ 
$M W_{\mathrm{O}_{2}}$ Molecular weight of oxygen

$M W_{o x}$ Molecular weight of metal oxide in oxidized form

$M W_{\text {red }}$ Molecular weight of metal oxide in reduced form

$n \quad$ Number of particles per unit volume or particle density.

$n_{i} \quad$ Number of moles of $\mathrm{i}^{\text {th }}$ species consumed

$\mathrm{n}_{\mathrm{A}} \quad$ Rate exponent for reactant species $\mathrm{A}$ and similar for other species

$N_{B} \quad$ Number of moles of species 'B' consumed per unit volume

$\dot{N}_{B} \quad$ Number of moles of species 'B' consumed per unit volume per unit time

$P \quad$ Gas pressure

$p_{i} \quad$ Pressure of the $\mathrm{i}^{\text {th }}$ phase

$p_{s} \quad$ Solids pressure

$p_{\text {friction }}$ Frictional pressure in the solid phase

$P_{\mathrm{H}_{2} \mathrm{O}} \quad$ Partial pressure of $\mathrm{H}_{2} \mathrm{O}$

$Q \quad$ Plastic potential

$r \quad$ Reaction rate $\left(\mathrm{kmol} /\left(\mathrm{m}^{3} . \mathrm{s}\right)\right)$

$r_{c} \quad$ Radius of the shrinking core

$\mathrm{R} \quad$ Universal gas constant. It is also the outer surface radius of the particle for shrinking core model and outer radius of the grain for the grain model

$R_{\mathrm{Fe} 2 \mathrm{O} 3}$ Percentage of $\mathrm{Fe}_{2} \mathrm{O}_{3}$ in the metal oxide particle

Re Reynolds number

$\vec{R}_{s g} \quad$ Momentum transfer between the $s^{\text {th }}$ solid phase and the gas phase

$R_{o} \quad$ Oxygen carrying capacity

$\{s\} \quad$ Deviatoric stress

$S_{0} \quad$ Initial particle surface area 
$\mathrm{t} \quad$ Time

$T_{\text {AR }} \quad$ Temperature in the air reactor

$T_{F R} \quad$ Temperature in the fuel reactor

$T_{\text {ref }} \quad$ Reference temperature

$T_{\text {inlet }} \quad$ Inlet air temperature

$u_{A R} \quad$ Fluidization velocity of the air reactor

$u_{F R} \quad$ Fluidization velocity in the fuel reactor

$u_{m f} \quad$ Minimum fluidization velocity of the particle

$u_{t} \quad$ Terminal velocity of the particle

$\vec{v}_{i} \quad$ Velocity of $\mathrm{i}^{\text {th }}$ phase

$\vec{v}_{g} \quad$ Velocity of gas phase

$\vec{v}_{s} \quad$ Velocity of solid phase

$\vec{v}_{q} \quad$ Velocity of $\mathrm{q}^{\text {th }}$ phase

$\vec{v}_{s g} \quad$ Relative velocity between gas phase and $s^{\text {th }}$ solid phase

$\vec{v}_{r s} \quad$ Relative velocity between $s^{\text {th }}$ and $r^{\text {th }}$ solid phase

$W_{o k} \quad$ Ultimate yield of the devolatiles

$W_{k} \quad$ Instantaneous mass of devolatiles already evolved.

$X_{A R} \quad$ Average conversion of metal oxide in the air reactor

$X_{F R} \quad$ Average conversion of metal oxide in fuel reactor

$\Delta X \quad$ Difference in conversion of metal oxide in AR and FR ( $\left.X_{A R}-X_{F R}\right)$

$Y_{B} \quad$ Mass fraction of species B

$Y_{D} \quad$ Mass fraction of species D

$Y_{i} \quad$ Mass fraction of the $\mathrm{i}^{\text {th }}$ species 
$Y_{i q} \quad$ Mass fraction of species $i$ in the $q^{\text {th }}$ phase

$\nabla \quad$ Gradient operator

$\beta_{s g} \quad$ The fluid-solid exchange coefficient

$\varepsilon_{i} \quad$ Volume fraction of the $\mathrm{i}^{\text {th }}$ phase

$\varepsilon_{g} \quad$ Volume fraction of gas phase

$\varepsilon_{0} \quad$ Initial porosity of the particle

$\varepsilon_{q} \quad$ Volume fraction of the $\mathrm{q}^{\text {th }}$ phase

$\varepsilon_{m f} \quad$ Volume fraction at minimum fluidization

$\varepsilon_{s} \quad$ Volume fraction of solid phase

$\varepsilon_{s, \text { min }}^{*} \quad$ Solid volume fraction at inception of frictional stress

$\varepsilon_{s, \text { max }}^{*}$ Solid volume fraction at maximum packing

$\gamma_{\Theta m} \quad$ Dissipation through collision

$\dot{\gamma} \quad$ Rate of strain

$\lambda_{\text {air }} \quad$ Air ratio (measure of extra air supplied)

$\lambda \quad$ Plastic multiplier

$\lambda_{i} \quad$ Bulk viscosity of the $i^{\text {th }}$ phase

$\lambda_{g} \quad$ Bulk velocity of the gas phase

$\lambda_{s} \quad$ Bulk velocity of the solid phase

$\eta_{C C} \quad$ Carbon capture efficiency

$\phi \quad$ Friction angle of granular particles

$\phi_{l s} \quad$ Dissipation in fluid

$\phi_{s} \quad$ Sphericity of the particle

$\sigma \quad$ Stress tensor 
$\sigma \quad$ Compressional hydrostatic stress

$\bar{\tau}_{g} \quad$ Gas phase stress tensor

$\tau \quad$ Reaction time scale

$\mu_{i} \quad$ Shear viscosity $\mathrm{i}^{\text {th }}$ phase

$\mu_{\text {friction }}$ Frictional viscosity in the solid phase

$\mu_{g} \quad$ Shear viscosity of gas phase

$\mu_{s} \quad$ Shear viscosity of solid phase

$\rho \quad$ Density of the $\mathrm{i}^{\text {th }}$ species

$\rho_{g} \quad$ Density of gas phase

$\rho_{s} \quad$ Density of solid phase

$\rho_{q} \quad$ Density of the $\mathrm{q}^{\text {th }}$ phase

$\bar{\tau}_{i} \quad$ Stress tensor

$=$

$\bar{\tau}_{g} \quad$ Gas phase stress tensor

$\bar{\tau}_{s} \quad$ Solid phase stress tensor

$\Theta \quad$ Granular temperature

$v_{i} \quad$ Stoichiometric coefficients of $\mathrm{i}^{\text {th }}$ species in a reaction 


\section{References}

Abad, A., T. Mattisson, A. Lyngfelt and M. Ryden, "Chemical-looping combustion in a 300W continuously operating reactor system using a manganese-based oxygen carrier," Fuel 85, 1174-1185, 2006.

Adánez, J., L. F. de Diego, F. García-Labiano, P. Gayán, and A. Abad, "Selection of oxygen carriers for chemical-looping combustion," Energy \& Fuels 18, 371-377, 2004.

Andrus, H.E., G. Burns, J.H. Chiu, G.N. Liljedahl, P.T. Stromberg, and P.R. Thibeault, "Hybrid combustiongasification chemical looping coal power technology", Development Phase III Final Report, Alstom Power Inc. PPL-08-CT-25, Contract DE-FC26-03NT41866, U.S. Department of Energy, National Energy Technology Laboratory, 2006.

Anheden, M., A.-S. Näsholm, and G. Svedberg,"Chemical-looping combustion - efficient conversion of chemical energy in fuels into work," IECEC Paper No. CT-45, Proceedings of Intersociety Energy Conversion Engineering Conference 3, 75-81, 1995.

Ansys Inc., "Fluent Users Manual Version 12.0," New Hampshire, USA, 2009.

Audus, H., O. Kaarstad and G. Skinner, " $\mathrm{CO}_{2}$ capture by pre-combustion decarbonisation of natural gas," Proceedings of the $4^{\text {th }}$ International Conference on Greenhouse Gas Control Technologies, Interlaken, 557-562, 1998.

Balaji S., Ilic, J., Erik Ydstie. B., Krogh, B. H., "Control-based modeling and simulation of the chemical looping combustion process," Ind. Eng. Chem. Res. 49, 4566-4575, 2010.

Berguerand, N. and A. Lyngfelt, "Design and operation of a $10 \mathrm{kWth}$ chemical-looping combustor for solid fuels - Testing with South African coal," Fuel 87, 2713-2726, 2008.

Berguerand, N. and A. Lyngfelt, "The use of petroleum coke as fuel in a $10 \mathrm{kWth}$ chemical-looping combustor," International Journal of Green House Gas Control 2, 169-179, 2008.

Bolland, O., and $\mathrm{H}$. Undrum, "Removal of $\mathrm{CO}_{2}$ from gas turbine power plants: evaluation of pre- and post combustion methods," Proceedings of the $4^{\text {th }}$ International Conference on Greenhouse Gas Control Technologies, Interlaken, 125-130, 1998.

Bradley, D., M. Lawes, H. Park and N. Usta, "Modeling of laminar pulverized coal flames with speciated devolatilization and comparisons with experiments," Combustion and Flame 144, 190-204, 2006.

Bustamante, F., R. M. Enick, A. Cugini, R. Killmeyer, B.H. Howard, K. S. Rothenberger, M. Ciocco, B. Morreale, S. Chattopadhyay and S. Shi, "Kinetic of the homogeneous reverse water-gas shift reaction at high temperature," AlChE Journal 50 (5), 1028 -1041, 2004. 
Chandel, M.K., A. Hoteit and A. Delebarre, "Experimental investigation of some metal oxides for chemical looping combustion in a fluidized bed reactor," Fuel 88, 898-908, 2009.

Chandrasekaran, B., L. Van der Lee, I. Hulme and A. Kantzas, "A simulation and experimental study of the hydrodynamics of a bubbling fluidized bed of linear low density polyethylene using bubble properties and pressure Fluctuations," Macromolecular Materials and Engineering 290 (6), 592 - 609, 2005.

Chiesa, P., S. Consonni and G. Lozza, "A comparative analysis of IGCCS with $\mathrm{CO}_{2}$ sequestration," Proceedings of the $4^{\text {th }}$ International Conference on Greenhouse Gas Control Technologies, Interlaken, 107-112, 1998.

Clift, R., Grace, J.R. (Eds.), “Continuous Bubbling and Slugging,” Academic Press, London, 1985.

Dartevelle, S., "Numerical and granulometric approaches to geophysical granular flows," Ph.D. thesis, Michigan Technological University, Department of Geological and Mining Engineering, Houghton, Michigan, July 2003.

De Diego, L.F., L. Garćia-Labiano, P. Gayán, J. Celaya, J.M. Palacios and J. Adánez, “Operation of a 10 $\mathrm{kW}_{\text {th }}$ chemical-looping combustor during $200 \mathrm{~h}$ with a $\mathrm{CuO}-\mathrm{Al}_{2} \mathrm{O}_{3}$ oxygen carrier," Fuel 86, 1036-1045, 2007.

Deng, Z., R. Xiao, B. Jin and Q. Song, "Numerical simulation of chemical looping combustion process with $\mathrm{CaSO}_{4}$ oxygen carrier," International Journal of Greenhouse Gas Control, Accepted for publication, 2009.

DeSouza-Santos, M., "Solid fuels combustion and gasification: Modeling, simulation and equipment operation," Marcel Deckker Inc., New York, 2004.

Drucker, D. C. and W. Prager, "Solid mechanics and plastic analysis for limit design," Quarterly of Applied Mathematics 10 (2), 157-165, 1952.

Everson, R., H. Neomagus, H. Kasaini and D. Njapha, "Reaction kinetics of pulverized coal-chars derived from inertinite-rich coal discards: Gasification with carbon dioxide and steam," Fuel 85, 1076-1082, 2006.

Fluent-Ansys Inc., "Fluent Users Manual Version 6.3," New Hampshire, USA, 2006.

Fryer, C. and O. Potter, "Experimental investigations of models for fluidized bed catalytic reactors", AIChE J. 22, 38, 1976.

Gao , Z., L. Shen, J. Xiao, C. Qing, Q. Song, "Use of coal as fuel for chemical looping combustion with NiBased Oxygen", Ind. Eng. Chem. Res. 47, 9279-9287, 2008. 
Gera, D., M. Syamlal, T. O'Brien, "Hydrodynamics of particle segregation in fluidized beds," International Journal of Multiphase Flow 30, 419-428, 2004.

Gidaspow, D., "Multiphase flow and fluidization," Academic Press, 1992.

Goldschmidt, M.J., J.M. Link, S. Mellema, J.A. Kuipers, "Digital image analysis measurements of bed expansion and segregation dynamics in dense gas-fluidised beds," Powder Technology 138, 135-159, 2003.

Guenther, C., M. Syamlal, L.J. Shadle, C. Ludlow, "A Numerical Investigation of an Industrial Scale GasSolids CFB", 7th International Circulating Fluid Bed Conference, Niagara Falls, Canada, 2002.

Haider, A and O. Levenspeil, "Drag coefficient and terminal velocity of spherical and nonspherical particles," Powder Tech. 58, 63, 1989.

Hulme, I., E. Clavelle, L. Van der Lee and A. Kantzas, "CFD modeling and validation of bubble properties for a bubbling fluidized Bed," Ind. Eng. Chem. Research 44 (12), 4254-4266, 2005.

Ishida, M., D. Zheng and T. Akehata, "Evaluation of a chemical-looping combustion power-generation system by graphic exergy analysis," Energy 12, 147-154, 1987.

Ishida, M., and H. Jin, "A fundamental study of a new kind of medium material for chemical-looping combustion," Chem. Eng. Jpn., 27, 296-301, 1994.

Ishida, M., and H. Jin, "Chemical looping combustor without NOx formation," Ind. Eng. Chem. 35, 24692472, 1996.

Jerndal, E., T. Mattisson and A. Lyngfelt, "Thermal analysis of chemical looping combustion," Chemical Engineering Research and Design 84, 795-806, 2006.

Johansson, M., T. Mattisson and A. Lyngfelt, "Investigation of $\mathrm{Fe}_{2} \mathrm{O}_{3}$ with $\mathrm{MgAl}_{2} \mathrm{O}_{4}$ for chemical looping combustion," Ind. Eng. Chem. 43, 6978-6987, 2004.

Johansson E., T. Mattisson, A. Lyngfelt and H. Thunman, "A 300W laboratory reactor system for chemical-looping combustion with particle circulation," Fuel 85, 1428-1438, 2006.

Johnson, $\mathrm{P}$ and R. Jackson, "Frictional-collisional constitutive relations for granular materials and applications to plain shearing," Journal of Fluid Mechanics 176, 67-93, 1987.

Jung, J., and I. Gamwo, "Multiphase CFD-based models for chemical looping combustion process: Fuel reactor modeling," Powder Technology 183, 401-409, 2008.

Kolbitsch, P., T. Pröll, J. Bolhar-Nordenkampf, and H. Hofbauer, "Characterization of chemical looping pilot plant performance via experimental determination of solids conversion," Energy \& Fuels 23, 14501455, 2009. 
Kronberger, B., E. Johansson, G. Loffler, T. Mattisson, A. Lyngfelt and H. Hofbauer, " A two compartment fluidized bed reactor for $\mathrm{CO}_{2}$ capture by chemical looping combustion", Chemical Engineering Technology 27, 2004.

Kronberger, B., G. Loffler and H. Hofbauer, "Simulation of mass and energy balances of a chemical looping combustion system", Clean air 6, 2005.

Knocke, K.F., and H. Richter, "Verbesserung der Reversibilität von Verbrennungsprozessen," BrennstoffWarme-Kraft 20, 205-210, 1968.

Kunnii, D. and O. Levenspiel, “Fluidization engineering," Butterworth-Heinmann publication, 1991.

Langroudi, L.M., S. Turek, A. Ouazzi and G.I. Tardos, “An investigation of frictional and collisional powder flows using a unified constitutive equation," Powder Technology 197 (1), 91-101, 2010.

Leion, H. and A. Lyngfelt, private communication, 2009.

Leion, H., T. Mattisson and A. Lyngfelt, "The use of petroleum coke as fuel in chemical-looping combustion," Fuel 86, 1947-1958, 2007.

Leion, H., T. Mattisson and A. Lyngfelt, "Solid fuels in chemical looping combustion," International Journal of Green House Gas Control 2, 180-193, 2008.

Levenspiel, O., “Chemical Reactor Engineering”, John Wiley and Sons, New York, 1962.

Liu, G., and S. Niksa, "Coal conversion submodels for design applications at elevated pressures: Part II Char gasification," Progress in Energy and Combustion Science 30, 2004.

Lun, C. K. K., S.B. Savage, D.J. Jeffrey and N. Chepurniy, "Kinetic theories for granular flow: inelastic particles in Couette flow and slightly inelastic particles in general flow field," J. Fluid Mech. 140, 223-256, 1984.

Lyngfelt, A., B. Lecknor and T. Mattisson, "A fluidized bed combustion process with inherent $\mathrm{CO}_{2}$ separation: An application of chemical looping combustion," Chem. Eng. Sci. 56, 3101-3113, 2001.

Lyngfelt A., and H. Thunman, "Chemical-looping combustion: Design, construction and $100 \mathrm{~h}$ of operational experience of a $10 \mathrm{~kW}$ prototype," Chapter 36 in The $\mathrm{CO}_{2}$ Capture and Storage Project (CCP) for Carbon Dioxide Storage in Deep Geologic Formations for Climate Change Mitigation, Volume 1 Capture and Separation of Carbon Dioxide from Combustion Sources, D. Thomas, ed., Elsevier Science, London, 2005.

Mao, D., J. Edwards, A. Kuznetsova and R. Srivastava, "Three-dimensional numerical simulation of a circulating fluidized bed reactor for multi-pollutant control", Chemical Engineering Science 59, 4279 4289, 2004. 
Marion, J.L., "Technology Options for Controlling $\mathrm{CO}_{2}$ Emissions from Fossil Fueled Power Plants," Fifth Ann. Conf. Carbon Capture and Sequestration, Alexandria, VA, May 8-10, 2006.

Mathiesen, V., T. Solberg and B. H. Hjertager,"An experimental and computational study of multiphase flow behavior in a circulating fluidized bed," International Journal of Multiphase Flow 26 (3), 387-419, 2000.

Mattisson, T., A. Lyngfelt and P. Cho, "The use of iron oxide as an oxygen carrier in chemical-looping combustion of methane with inherent separation of $\mathrm{CO}_{2}, "$ Fuel 80, 1953-1962, 2001.

Mattisson, T., J. Abanades A. Lyngfelt, A. Abad, M. Johansson, J. Adanez, F. Garcia-Labiano, L.F de Diego, P. Gayan, B. Kronberger, H. Hofbauer, M. Luisser, J.M. Palacios, D. Alvares, M. Orjala and V.P. Heiskanen , "Capture of $\mathrm{CO}_{2}$ in coal combustion (CCCC)," ECSC Coal RTD Programme Final Report, ECSC-7220PR125, 2005.

Merrick, D., "Mathematical models for thermal decomposition of coal- The evolution of volatile matter," Fuel 62, 1983.

Moritomi, H., T. Iwase and T. Chiba, "A comprehensive interpretation of solid layer inversion in liquidfluidized beds," Chemical Engineering Science 37, 1751-1757, 1982.

O'Brien, T.J. and M. Syamlal, "Particle cluster effects in the numerical simulation of a circulating fluidized bed," in Circulating Fluidized Bed Technology IV, Ed. A. Avidan, Proceedings of the Fourth International Conference on Circulating Fluidized Beds, Somerset, PA, August 1-5, 1993.

Ocone, R., S. Sundaresan, and R. Jackson, "Gas-particle flow in a duct of arbitrary inclination with particle-particle interaction," AIChE J. 39,1261-1271, 1993.

Ogawa, S., A. Umemura and N. Oshima, "On the equations of fully fluidized granular materials," $Z$. Angew. Math. Phys, 31, 483 - 493, 1980.

Pitman, B. and D. Schaeffer, "Stability of time dependent compressible granular flows in two dimesions," Comm Pure Applied Mathematics 40, 421-447, 1987.

Richter, H.J., and K.F. Knoche, "Reversibility of combustion processes," ACS Symp. Series 235, 71-85, 1983.

Ryu, H., D. Bae, K. Han, S. Lee, G. Jin and J. Choi, "Oxidation and reduction characteristics of oxygen carrier particles and reaction kinetics by unreacted core model," Korean J. Chem. Eng. 18, 831-837, 2001.

Samuelsberg, A. and B. H. Hjertager, "An experimental and numerical study of flow patterns in a circulating fluidized bed reactor". Int. J. Multiphase Flow 22 (3), 575-591, 1996. 
Savage, S, B. and M. Sayed, "Stresses developed by dry cohesionless granular materials sheared in an annular shear cell," Journal of Fluid Mechanics 142, 391-430, 1984.

Schaeffer, D, G., "Instability in the evolution equations describing incompressible granular flow," Journal of Differential Equations 66, 19-50, 1987.

Shen, L., J. Wu and J. Xiao, "Experiments on chemical looping combustion of coal with a NiO based oxygen carrier," Combustion and Flame, 2009.

Shen, L., J. Wu, J. Xiao, Q. Song and R. Xiao, "Chemical-Looping combustion of biomass in a $10 \mathrm{kWth}$ reactor with iron oxide as an oxygen carrier," Energy and Fuels, 23, 2498-2505, 2009.

Shi, J., E. Donskoi and L. McElwain, "Modeling novel coal based direct reduction process," Iron Making and Steel Making, 2008.

Simbeck, D.,"A portfolio selection approach for power plant $\mathrm{CO}_{2}$ capture, separation and R\&D options," Proceedings of the $4^{\text {th }}$ International Conference on Greenhouse Gas Control Technologies, Interlaken, 119-124, 1998.

Siriwardane, R., J. Poston, K. Chaudhari, A. Zinn, T. Simonyi and C. Robinson, "Chemical-looping combustion of simulated synthesis gas using nickel oxide oxygen carrier supported on bentonite," Energy \& Fuels 21, 1582-1591, 2007.

Smoot, L. D. and P. J. Smith. “Coal Combustion and Gasification," Plenum Press, New York, 1985.

Son, S. and S. Kim, "Chemical-Looping combustion with $\mathrm{NiO}$ and $\mathrm{Fe}_{2} \mathrm{O}_{3}$ in a thermo balance and circulating fluidized bed reactor," Industrial Eng. Chem. 45, 2689-2696, 2006.

Srivastava, A. and S. Sundaresan, "Analysis of a frictional-kinetic model for gas-particle flow," Powder Technology 129, 72-85, 2003.

Syamlal, M. and T. O'Brien, "Simulation of granular layer inversion in liquid fluidized bed," Int. J. of Multiphase Flows, 14 (4), 1988.

Syamlal, M., W. Rogers, and T. O'Brien, "MFIX documentation and theory guide," US-Department of Energy Report No. DOE/METC-94/1004, 1993.

Syamlal, M. and Bissett, L., "METC gasifier advanced simulation model," US-Department of Energy Report No. DOE/METC-92/4108, 1992.

Szekely, J., W. Evans and H. Sohn, “Gas-Solid Reactions," Academic Press, 1976.

Taylor, G, I., "The dispersion of matter in turbulent flow through pipes," Proceedings of the Royal Society of London. Series A, Mathematical and Physical Sciences 223, 1155, 1954.

Wen, C, Y. and Yu, H, Y., "Mechanics of fluidization," Chem. Eng. Prog. Symp. Series. 62, 100, 1966. 
Wolf, J., M. Anheden and J. Yan, "Performance analysis of combined cycles with chemical looping combustion for $\mathrm{CO}_{2}$ capture," Proc. 18th Int. Pittsburgh Coal Conf., 1122-1139, 2001. 


\section{APPENDIX 1: Detailed calculations for volatile composition and gasification products for South African Coal}

Merrick (1983) suggests that the total volatile released will be given as:

$V=p-0.36 p^{2}$

Where $p=$ volatile matter from proximate analysis.

Table 1: Details of South African coal.

\begin{tabular}{|l|l|}
\hline & Mass \% \\
\hline Volatile & 21.6 \\
\hline Moisture & 8.3 \\
\hline Ash & 15.9 \\
\hline Fixed Carbon & 54.2 \\
\hline
\end{tabular}

\begin{tabular}{|l|l|}
\hline & $\begin{array}{l}\text { Mass in terms of total } \\
\text { coal\% }\end{array}$ \\
\hline C & 62.5 \\
\hline H & 3.5 \\
\hline S & 0.7 \\
\hline N & 1.4 \\
\hline O & 7.7 \\
\hline
\end{tabular}

Table 2: Details of South African coal composition without ash and moisture.

\begin{tabular}{|c|c|c|c|}
\hline & Mass $\%$ & & $\begin{array}{l}\text { Mass in terms of } \\
\text { daf coal } \%\end{array}$ \\
\hline Fixed Carbon & 71.504 & $\mathrm{C}$ & 82.4538 \\
\hline \multirow[t]{4}{*}{ Volatile } & 28.496 & $\mathrm{H}$ & 4.6174 \\
\hline & & $S$ & 0.9235 \\
\hline & & $\mathrm{N}$ & 1.847 \\
\hline & & 0 & 10.1583 \\
\hline
\end{tabular}

Table 3: Characteristics of Char and Tar (Bradley et al., 2005 and Merrick., 1983).

\begin{tabular}{|c|c|c|c|c|c|c|c|}
\hline & $\mathrm{C}$ & $\mathrm{H}$ & 0 & $\mathrm{~N}$ & $\mathrm{~S}$ & MW & Composition \\
\hline Char & 0.98 & 0.002 & 0.002 & 0.01 & 0.006 & 12.245 & $\mathrm{CH}_{0.0245} \mathrm{O}_{0.001531} \mathrm{~N}_{0.00875} \mathrm{~S}_{0.0023}$ \\
\hline Tar & 0.85 & 0.082 & 0.049 & 0.009 & 0.01 & 14.1174 & $\mathrm{CH}_{1.158} \mathrm{O}_{0.0432} \mathrm{~N}_{0.0091} \mathrm{~S}_{0.0044}$ \\
\hline
\end{tabular}


The total volatile mass $\%$ is given as

$$
V=p-0.36 p^{2}=0.285-0.36 \times(0.285)^{2}=0.2558
$$

The Char mass percentage is calculated as:

$$
C H A R=1-V=1-0.2558=0.7442
$$

Based on assumption 1 above we can calculate the $\mathrm{CH}_{4}$ mass $\%$ as:

$$
\mathrm{CH}_{4}=H \times 0.327 \times \frac{16}{4}=1.308 H=1.308 \times 0.046174=0.0604
$$

Based on assumption 2 above we can calculate the $\mathrm{C}_{2} \mathrm{H}_{6}$ mass \% as:

$$
C_{2} H_{6}=H \times 0.044 \times \frac{30}{6}=0.22 H=0.22 \times 0.046174=0.0101
$$

Based on assumption 3 above we can calculate the CO mass \% as:

$$
C O=O \times 0.185 \times \frac{28}{16}=0.324 O=0.324 \times 0.101583=0.03291
$$

Based on assumption 4 above we can calculate the $\mathrm{CO} 2$ mass $\%$ as:

$$
C O_{2}=O \times 0.11 \times \frac{44}{32}=0.15 O=0.15 \times 0.101583=0.01524
$$

The carbon balance gives:

$0.98 \times C H A R+0.85 \times T A R+\frac{12}{16} \mathrm{CH}_{4}+\frac{24}{30} \mathrm{C}_{2} \mathrm{H}_{6}+\frac{12}{28} \mathrm{CO}+\frac{12}{44} \mathrm{CO}_{2}=\mathrm{C}$

$0.85 \times T A R=C-\left(0.98 \times C H A R+\frac{12}{16} \mathrm{CH}_{4}+\frac{24}{30} \mathrm{C}_{2} \mathrm{H}_{6}+\frac{12}{28} \mathrm{CO}+\frac{12}{44} \mathrm{CO}_{2}\right)$

$T A R=\frac{1}{0.85}\left\lfloor 0.8245-\left(0.98 \times 0.7442+\frac{12}{16} \times 0.0604+\frac{24}{30} \times 0.0101+\frac{12}{28} \times 0.03291+\frac{12}{44} \times 0.01524\right)\right\rfloor$

$T A R=0.0277$

The sulfur balance gives:

$0.006 \times C H A R+0.01 \times T A R+\frac{32}{34} H_{2} S=S$ 


$$
\begin{aligned}
& H_{2} S=\frac{34}{32}(S-0.006 \times C H A R-0.01 \times T A R) \\
& H_{2} S=\frac{34}{32}(0.009235-0.006 \times 0.7442-0.01 \times 0.0277) \\
& H_{2} S=0.0047736
\end{aligned}
$$

The Nitrogen balance gives:

$0.01 \times C H A R+0.009 \times T A R+\frac{14}{17} N H_{3}=N$

$\mathrm{NH}_{3}=\frac{17}{14}(N-0.01 \times C H A R-0.009 \times T A R)$

$\mathrm{NH}_{3}=\frac{17}{14}(0.01847-0.01 \times 0.7442-0.009 \times 0.0277)$

$N H_{3}=0.0131$

The Oxygen balance gives:

$$
\begin{aligned}
& 0.002 \times C H A R+0.049 \times T A R+\frac{16}{28} \mathrm{CO}+\frac{32}{44} \mathrm{CO}_{2}+\frac{16}{18} \mathrm{H}_{2} \mathrm{O}=\mathrm{O} \\
& \mathrm{H}_{2} \mathrm{O}=\frac{18}{16}\left(\mathrm{O}-0.002 \times \mathrm{CHAR}-0.049 \times \mathrm{TAR}-\frac{16}{28} \mathrm{CO}-\frac{32}{44} \mathrm{CO}_{2}\right) \\
& \mathrm{H}_{2} \mathrm{O}=\frac{18}{16}\left(0.101583-0.002 \times 0.7442-0.049 \times 0.0277-\frac{16}{28} 0.03291-\frac{32}{44} 0.01524\right) \\
& \mathrm{H}_{2} \mathrm{O}=0.07745
\end{aligned}
$$

The Hydrogen balance gives:

$$
\begin{aligned}
& 0.002 \times C H A R+0.082 \times T A R+\frac{4}{16} \mathrm{CH}_{4}+\frac{6}{30} \mathrm{C}_{2} H_{6}+H_{2}+\frac{3}{17} \mathrm{NH}_{3}+\frac{2}{18} H_{2} \mathrm{O}+\frac{2}{34} \mathrm{H}_{2} S=H \\
& H_{2} \mathrm{O}=\frac{18}{2}\left(H-0.002 \times C H A R-0.082 \times T A R-\frac{4}{16} \mathrm{CH}_{4}-\frac{6}{30} \mathrm{C}_{2} H_{6}-H_{2}-\frac{3}{17} N H_{3}-\frac{2}{34} H_{2} S\right) \\
& H_{2}=\left(0.04617-0.002 \times 0.7442-0.082 \times 0.0277-\frac{4}{16} 0.0604-\frac{6}{30} 0.0101-\frac{2}{18} 0.07745-\frac{3}{17} 0.0131-\frac{2}{34} 0.004774\right) \\
& H_{2}=0.0141
\end{aligned}
$$


Calculation of Molecular weight of coal is calculate based on elemental composition as follows:

$$
\begin{aligned}
& M W_{-} d a f_{-} \text {coal }=\frac{1}{\frac{C}{12}+\frac{H}{1}+\frac{O}{16}+\frac{N}{14}+\frac{S}{32}} \\
& M W_{-} d a f_{-} \text {coal }=\frac{1}{\frac{0.8245}{12}+\frac{0.04617}{1}+\frac{0.1015}{16}+\frac{0.01847}{14}+\frac{0.009235}{32}}=8.14 \frac{\mathrm{kg}}{\mathrm{kmole}}
\end{aligned}
$$

Accounting for Ash+Moisture and assuming the molecular weight of Ash is $100 \mathrm{~kg} / \mathrm{kmol}$ we have

$$
M W_{-} \text {Coal }=\frac{1}{\frac{0.758}{8.14}+\frac{0.159}{100}+\frac{0.083}{18}}=10.07 \frac{\mathrm{kg}}{\mathrm{kmole}}
$$

Therefore we have the following mass balance:

$$
\begin{gathered}
1 \mathrm{~kg}(\text { daf_Coal }) \rightarrow 0.7442 \mathrm{CHAR}+0.0277 T A R+0.0604 \mathrm{CH}_{4}+0.0101 \mathrm{C}_{2} \mathrm{H}_{6}+0.03291 \mathrm{CO}+0.01524 \mathrm{CO}_{2}+ \\
+0.0141 \mathrm{H}_{2}+0.0131 \mathrm{NH}_{3}+0.07745 \mathrm{H}_{2} \mathrm{O}+0.004774 \mathrm{H}_{2} \mathrm{~S}(\mathrm{~kg})
\end{gathered}
$$

Considering the ash and moisture in the coal we have the following mass balance

$$
\begin{aligned}
0.758 \mathrm{~kg}(\text { daf_Coal })+0.159 \mathrm{ASH}+0.083 \text { Moisture } \rightarrow & 0.564 \mathrm{CHAR}+0.0209 \mathrm{TAR}+0.0458 \mathrm{CH}_{4}+0.0077 \mathrm{C}_{2} \mathrm{H}_{6} \\
& +0.025 \mathrm{CO}+0.0116 \mathrm{CO}_{2}+0.0107 \mathrm{H}_{2}+0.0099 \mathrm{NH}_{3} \\
& +0.0586 \mathrm{H}_{2} \mathrm{O}+0.0036 \mathrm{H}_{2} \mathrm{~S}+0.159 \mathrm{ASH}+0.083 \text { Moisture }(\mathrm{kg})
\end{aligned}
$$

Now using Table 4 we have the MW of CHAR and TAR as 12.245 and 14.1174. The MW of ASH is assumed to be 100 .

Therefore we have the following mole balance

$$
\begin{aligned}
\frac{1}{10.07} \operatorname{Coal}(\text { kmoles }) \rightarrow & 0.0461 \mathrm{CHAR}+0.00148 T A R+0.00286 \mathrm{CH}_{4}+0.00025 \mathrm{C}_{2} \mathrm{H}_{6}+0.00089 \mathrm{CO}+0.000264 \mathrm{CO}_{2} \\
& \left.+0.00534 \mathrm{H}_{2}+0.000583 \mathrm{NH}_{3}+0.00325 \mathrm{H}_{2} \mathrm{O}+0.000106 \mathrm{H}_{2} \mathrm{~S}+0.00159 \mathrm{ASH}+0.00461 \text { Moisture (kmoles }\right)
\end{aligned}
$$

$$
\begin{array}{r}
\text { Coal }(\text { kmoles }) \rightarrow 0.4639 \mathrm{CHAR}+0.0149 \mathrm{TAR}+0.0288 \mathrm{CH}_{4}+0.0026 \mathrm{C}_{2} \mathrm{H}_{6}+0.00896 \mathrm{CO}+0.00266 \mathrm{CO}_{2}+0.054 \mathrm{H}_{2} \\
+0.0059 \mathrm{NH}_{3}+0.0328 \mathrm{H}_{2} \mathrm{O}+0.0011 \mathrm{H}_{2} \mathrm{~S}+0.0165 \mathrm{ASH}+0.0477 \text { Moisture }(\mathrm{kmoles})
\end{array}
$$

(18) 


\section{Secondary Devolatilization of TAR:}

Bradley et al., 2005 suggest that the decomposition of TAR results in soot, $\mathrm{CH} 4, \mathrm{CO}, \mathrm{H} 2$ and $\mathrm{HCN}$. Bradley et al. exclude sulfur in their analysis. To account for sulfur we add H2S.

$$
\operatorname{Tar}\left(\mathrm{CH}_{1.158} \mathrm{O}_{0.0432} \mathrm{~N}_{0.009} \mathrm{~S}_{0.044}\right) \rightarrow q \underset{\text { Soot }}{\mathrm{C}}+\alpha \mathrm{CH}_{4}+\beta \mathrm{CO}+\gamma \mathrm{HCN}+\delta \mathrm{H}_{2}+\lambda \mathrm{H}_{2} \mathrm{~S}
$$

Therefore $\gamma=0.0091, \lambda=0.0044$ and $\beta=0.0432$

They also assume that the ratio of $\mathrm{H} 2$ and $\mathrm{CH} 4$ is the same as in primary devolatilization.

$\alpha / \delta=0.0297 / 0.059=0.5$

$4 \alpha+2 \delta+\gamma+2 \lambda=1.158$

Therefore $\alpha=0.1425$ and $\delta=0.285$

$q=1-\alpha-\beta-\gamma=0.8052$

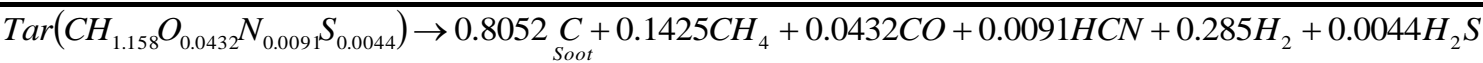

Coal (kmoles $) \rightarrow 0.4639 \mathrm{CHAR}^{2} 0.0149 \mathrm{TAR}+0.0288 \mathrm{CH}_{4}+0.0026 \mathrm{C}_{2} \mathrm{H}_{6}+0.00896 \mathrm{CO}+0.00266 \mathrm{CO}_{2}+0.054 \mathrm{H}_{2}$ $+0.0059 \mathrm{NH}_{3}+0.0328 \mathrm{H}_{2} \mathrm{O}+0.0011 \mathrm{H}_{2} \mathrm{~S}+0.0165 \mathrm{ASH}+0.0477$ Moisture (kmoles)

Primary + Secondary Devolatilization gives:

Coal $(\mathrm{kmoles}) \rightarrow 0.4639 \mathrm{CHAR}+0.012 \underset{\text { Soot }}{\mathrm{C}+0.0309 \mathrm{CH}_{4}+0.0026 \mathrm{C}_{2} \mathrm{H}_{6}+0.0096 \mathrm{CO}+0.00266 \mathrm{CO}_{2}+0.0582 \mathrm{H}_{2}}$ $+0.0059 \mathrm{NH}_{3}+0.0328 \mathrm{H}_{2} \mathrm{O}+0.0011 \mathrm{H}_{2} \mathrm{~S}+0.00013 \mathrm{HCN}+0.0165 \mathrm{ASH}+0.0477$ Moisture(kmoles)

\section{Gassification of CHAR:}

The composition of CHAR is given as $\mathrm{CH}_{0.0245} \mathrm{O}_{0.001531} \mathrm{~N}_{0.00875} \mathrm{~S}_{0.0023}$

The fate of Sulfur, Nitrogen, Oxygen etc is not clear, therefore here we make assumptions on formation of $\mathrm{H} 2 \mathrm{O}, \mathrm{H} 2 \mathrm{~S}$ and $\mathrm{N} 2$

Char $\left(\mathrm{CH}_{0.0245} \mathrm{O}_{0.00153} \mathrm{~N}_{0.0087} \mathrm{~S}_{0.0023}\right)+\mathrm{H}_{2} \mathrm{O} \rightarrow \mathrm{CO}+1.00842 \mathrm{H}_{2}+0.004375 \mathrm{~N}_{2}+0.001531 \mathrm{H}_{2} \mathrm{O}+0.0023 \mathrm{H}_{2} \mathrm{~S}$

Char $\left(\mathrm{CH}_{0.0245} \mathrm{O}_{0.00153} \mathrm{~N}_{0.00875} \mathrm{~S}_{0.0023}\right)+\mathrm{CO}_{2} \rightarrow 2 \mathrm{CO}+0.00842 \mathrm{H}_{2}+0.004375 \mathrm{~N}_{2}+0.001531 \mathrm{H}_{2} \mathrm{O}+0.0023 \mathrm{H}_{2} \mathrm{~S}$ 


\section{APPENDIX 2: User Defined Functions for Coal Combustion Studies}

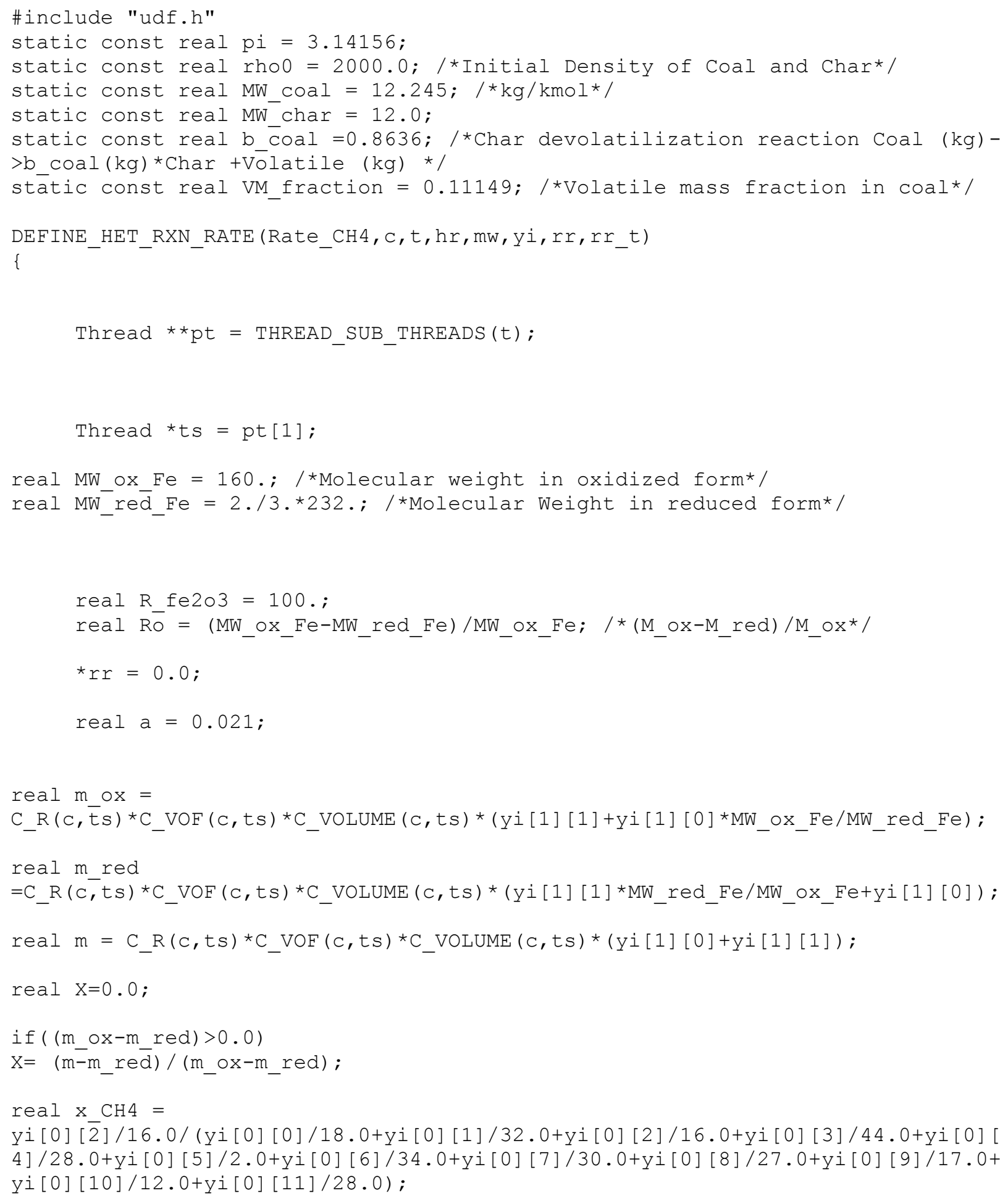




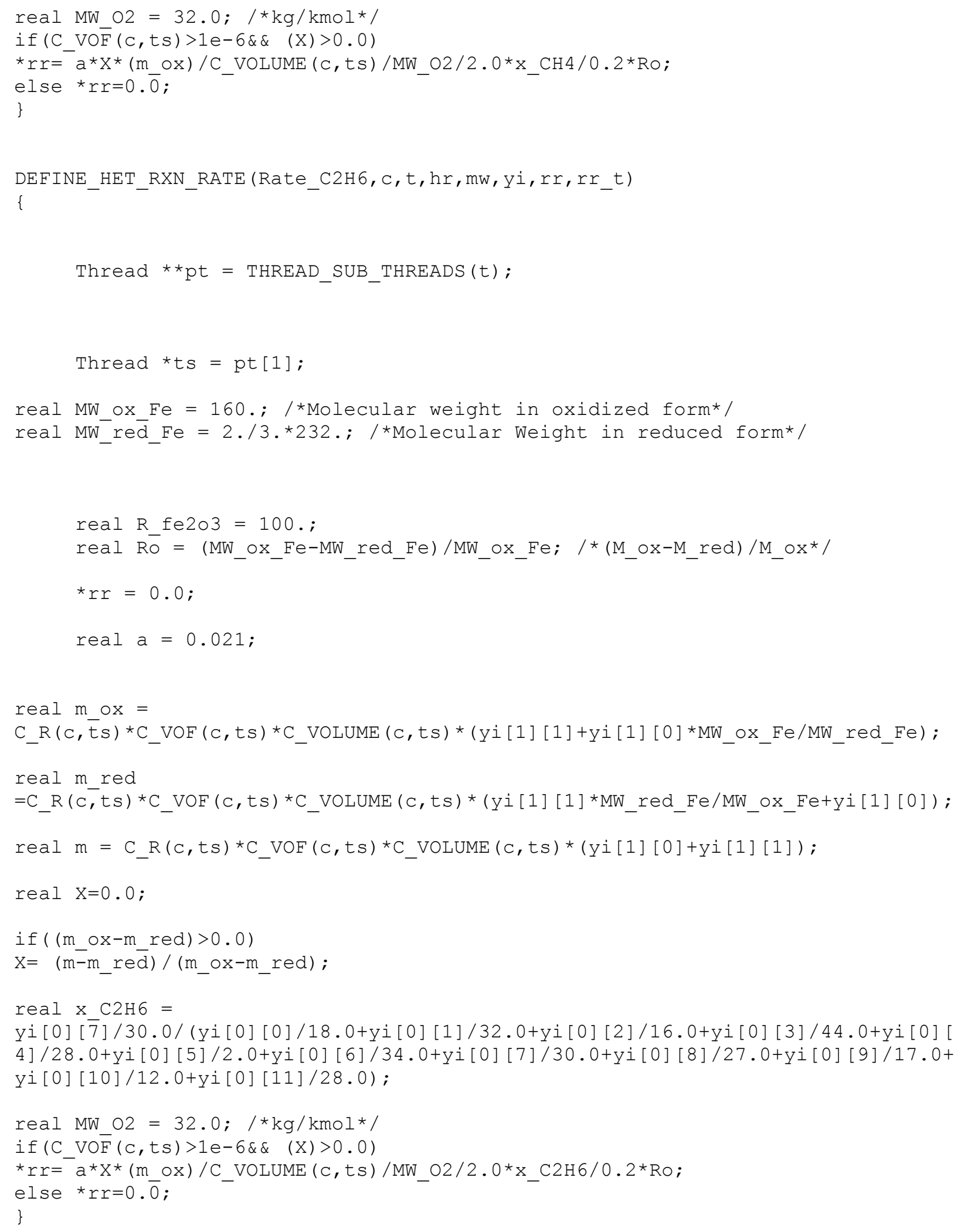




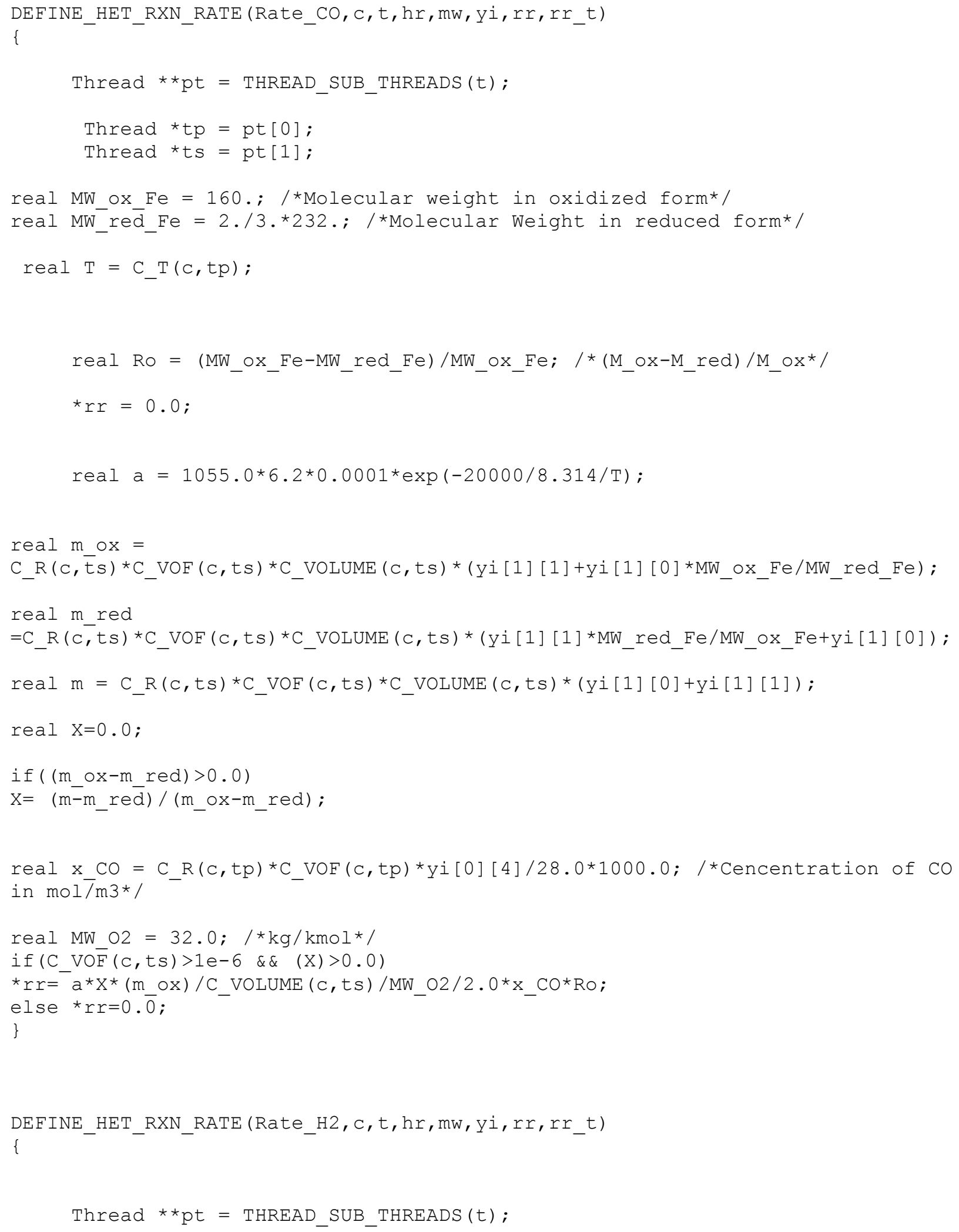




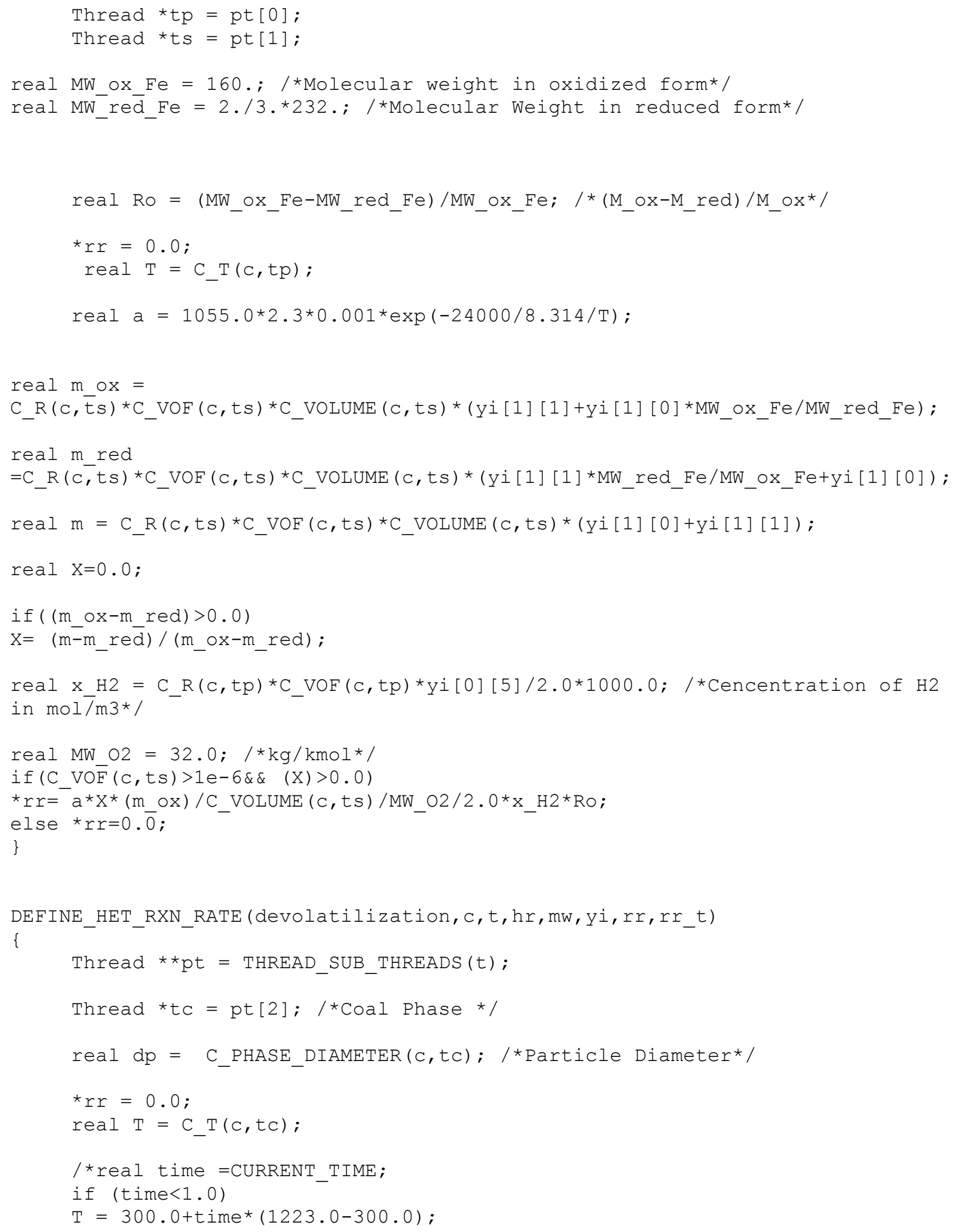




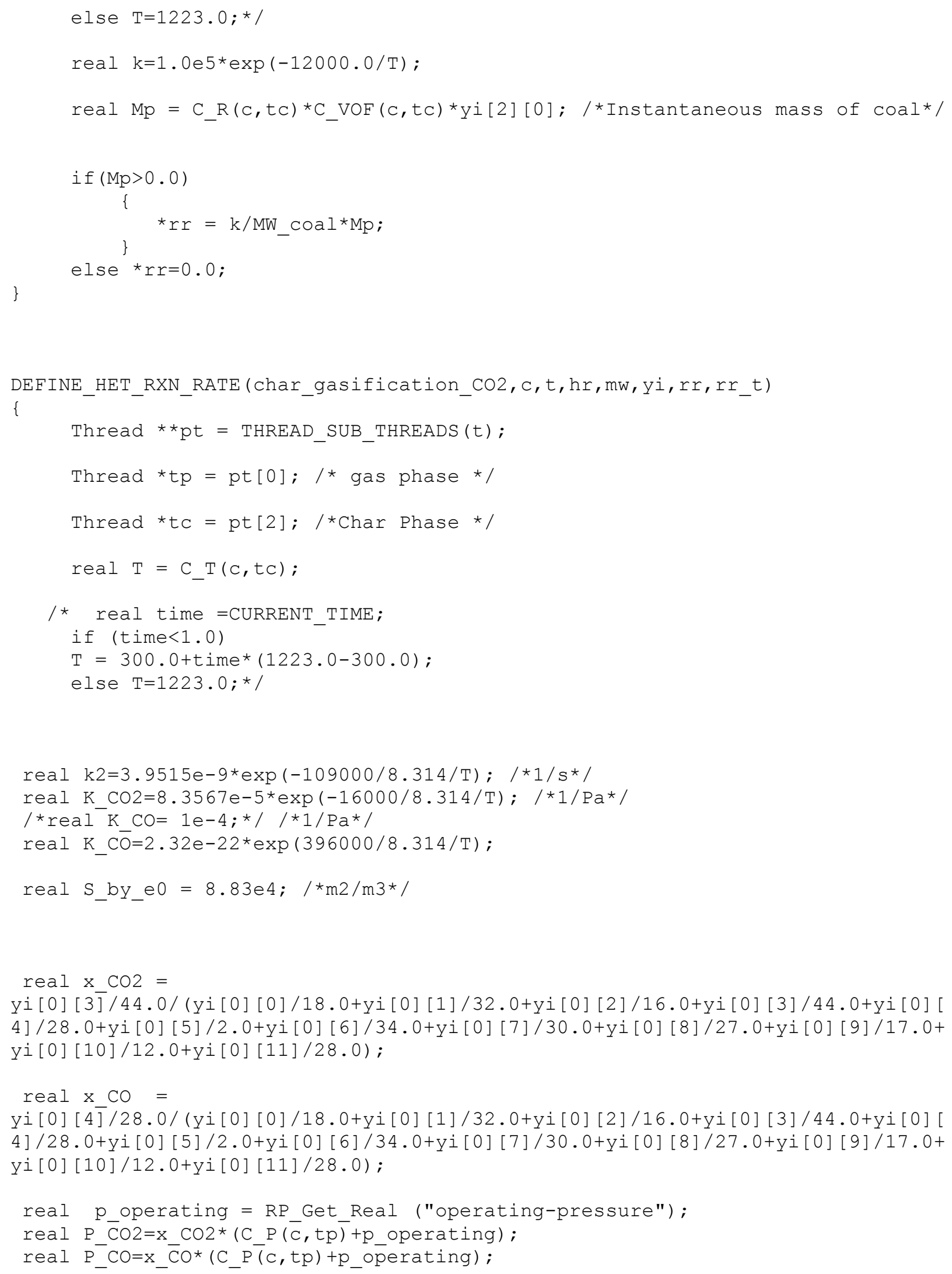




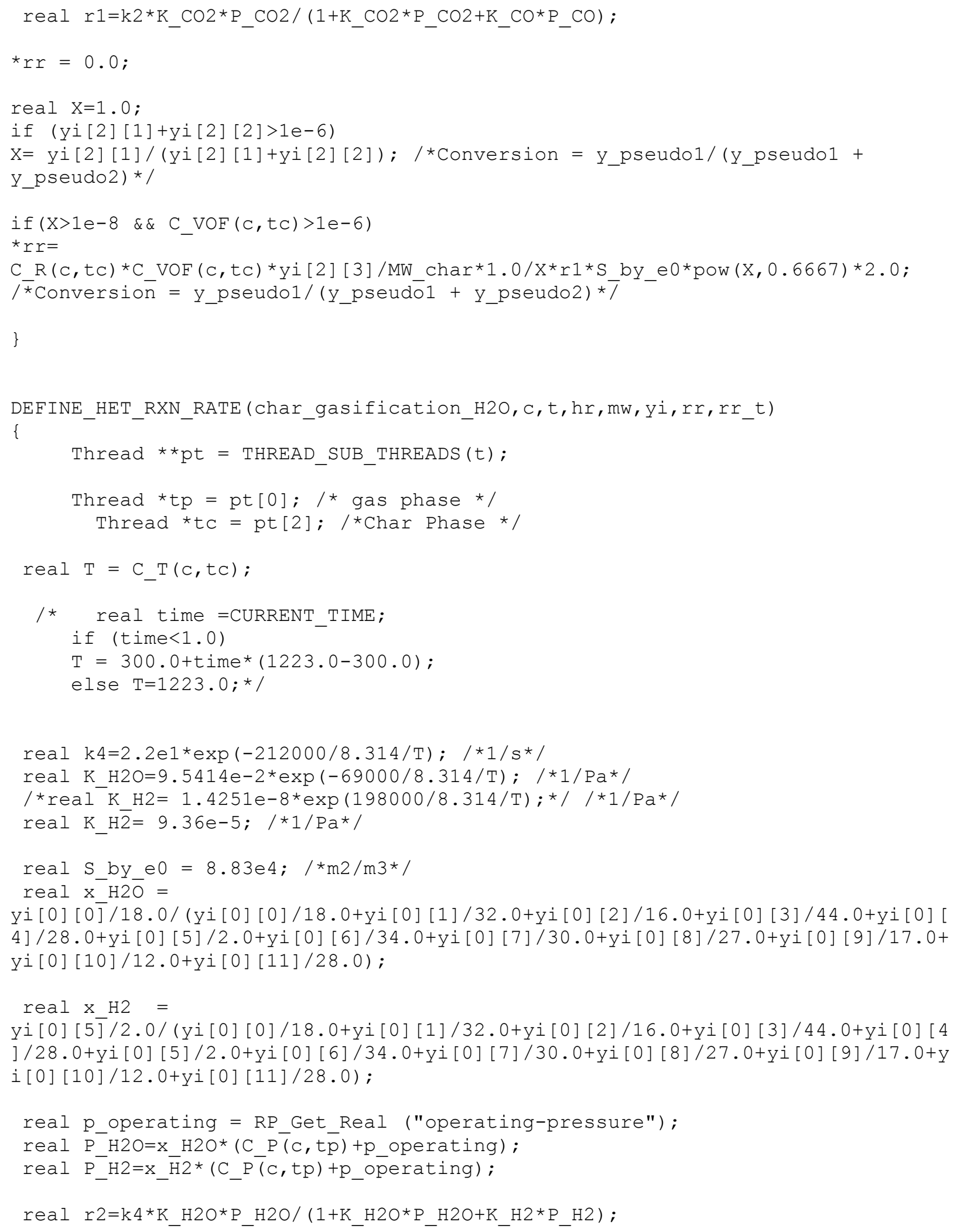




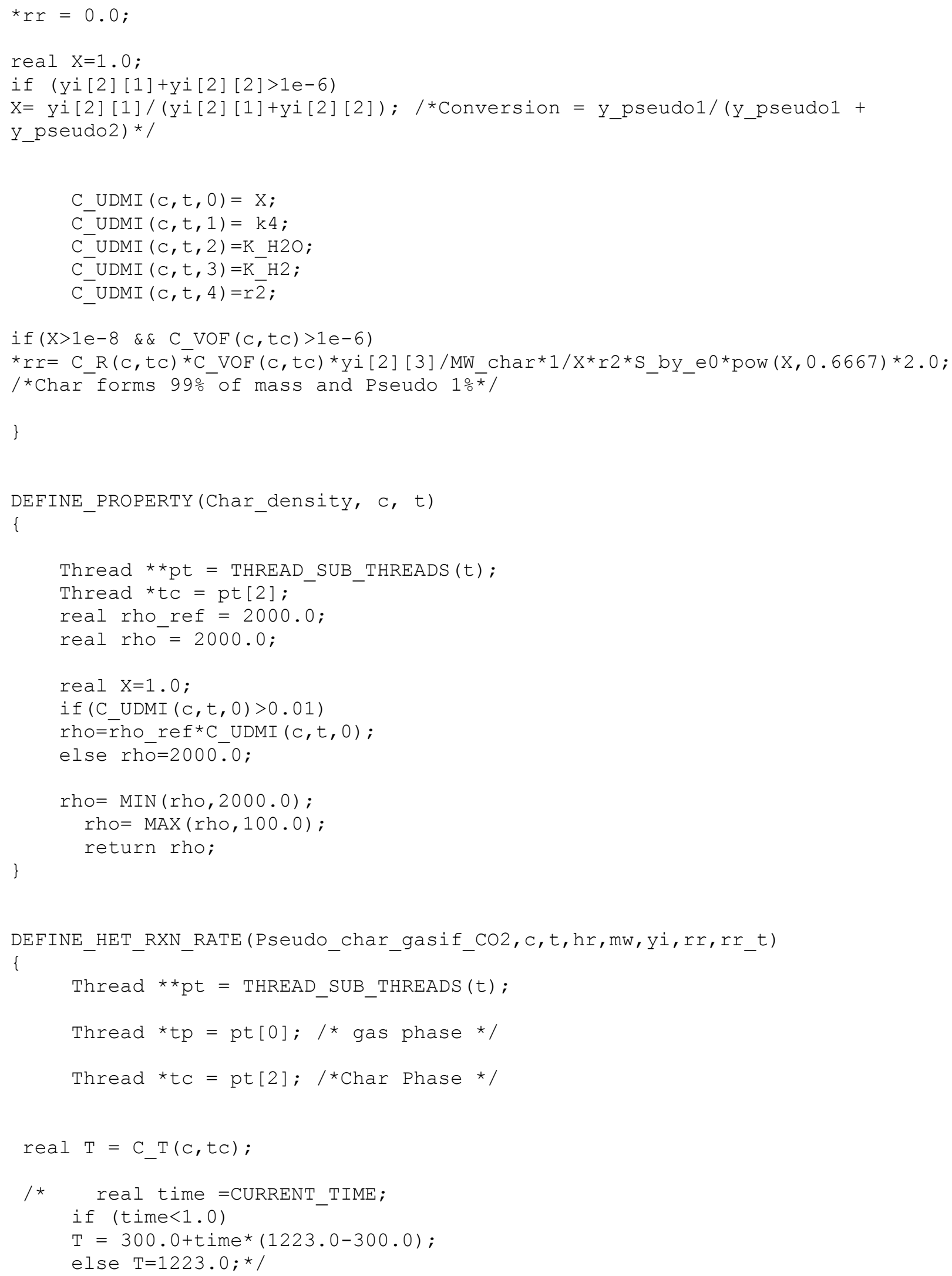




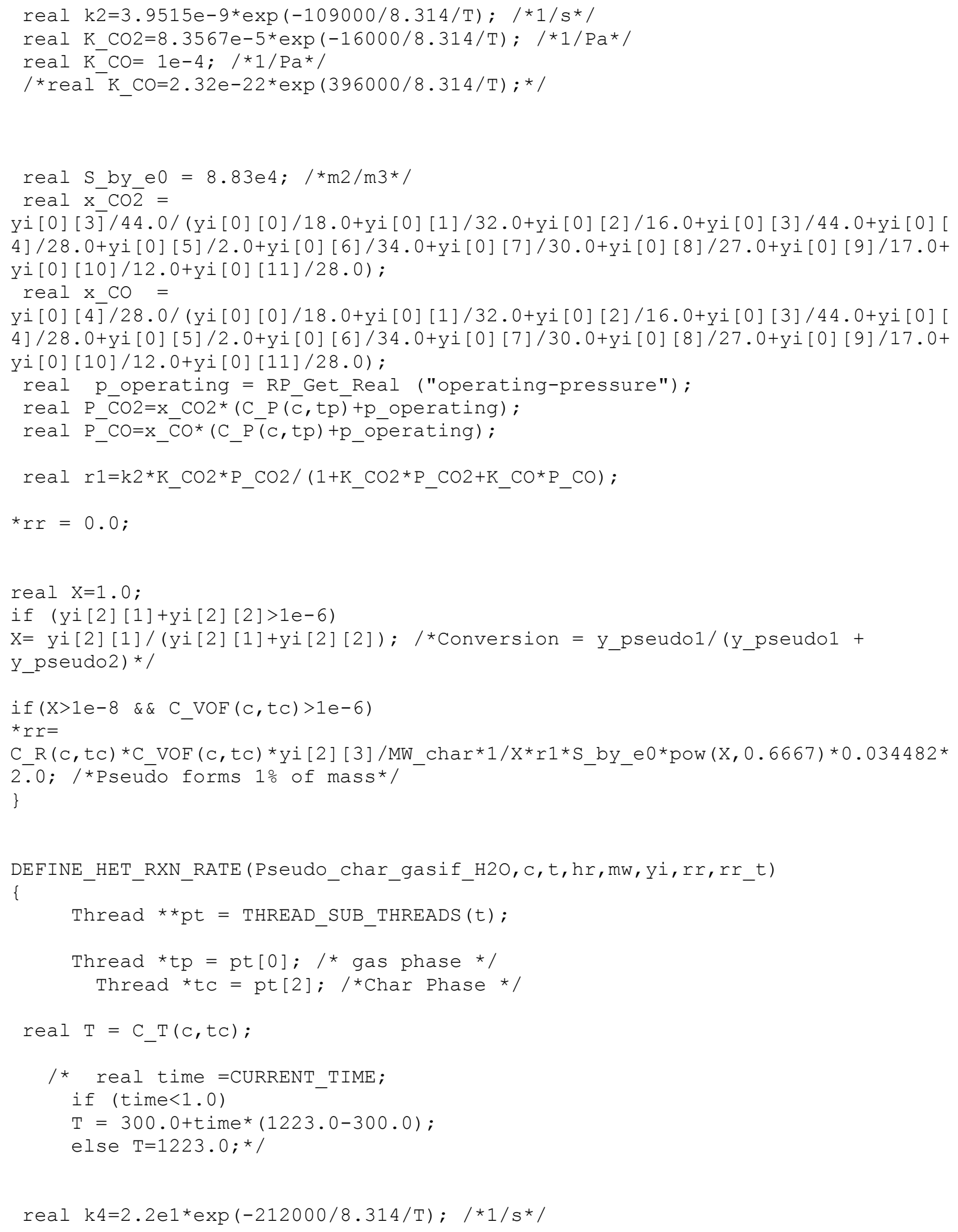




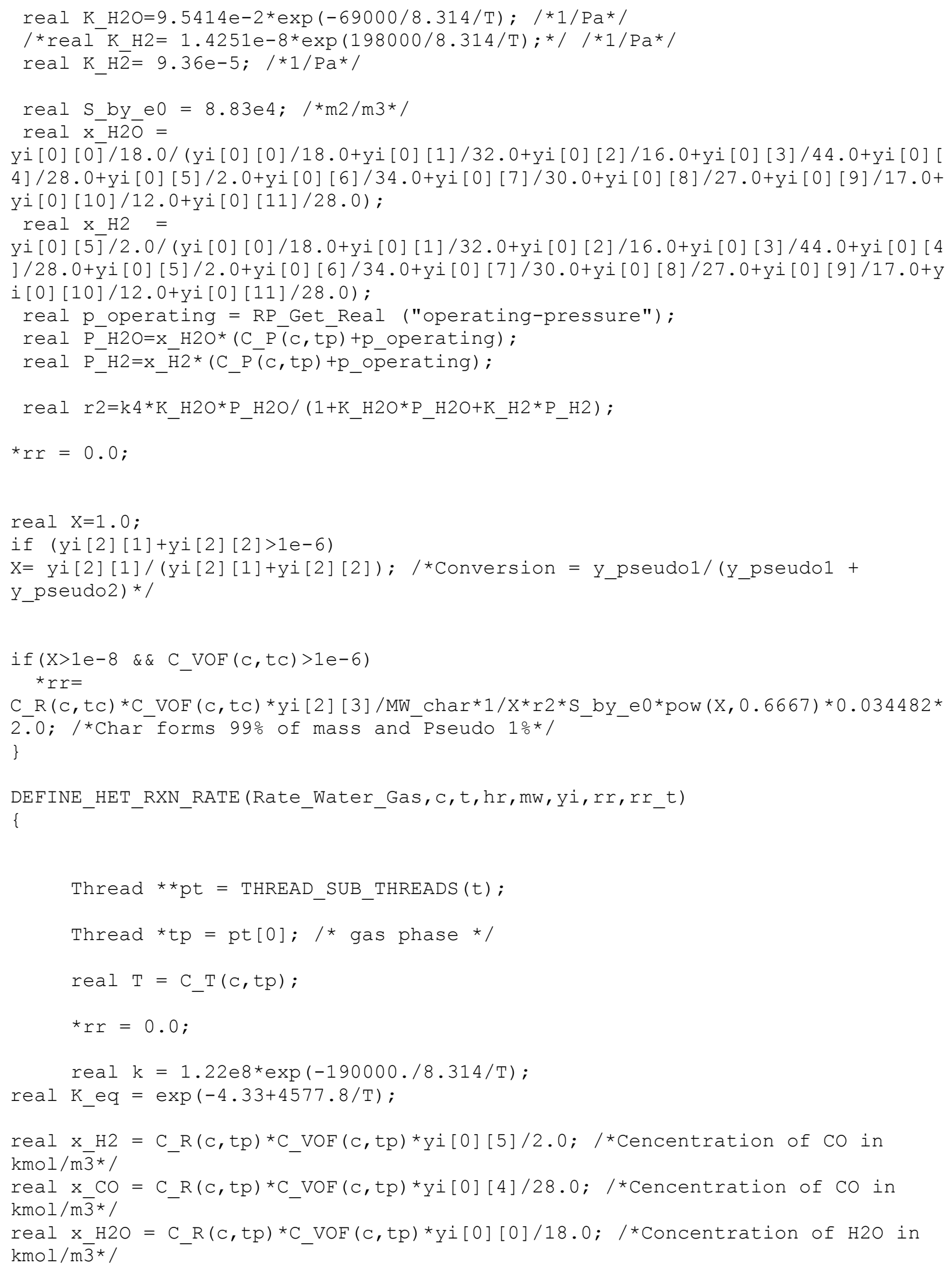


real $x_{-} \mathrm{CO} 2=\mathrm{C}_{-} \mathrm{R}(\mathrm{c}, \mathrm{tp}){ }^{*} \mathrm{C}_{-} \operatorname{VOF}(\mathrm{c}, \mathrm{tp}){ }^{*} \mathrm{yi}[0][3] / 44.0 ; /{ }^{*}$ Concentration of $\mathrm{H} 2 \mathrm{O}$ in $\mathrm{kmol} / \mathrm{m} \overline{3} * /$

${ }^{*} r r=-\left(\mathrm{k} * x_{-} \mathrm{CO} 2 *\right.$ pow $\left.\left(\mathrm{x}_{-} \mathrm{H} 2,0.5\right)-\mathrm{k} / \mathrm{K}_{-} \mathrm{eq}{ }^{*} \mathrm{x}_{-} \mathrm{CO} \mathrm{x}_{-} \mathrm{H} 2 \mathrm{O}\right) ;$

\} 


\section{APPENDIX 3: User Defined Functions for Gaseous Fuel Combustion}

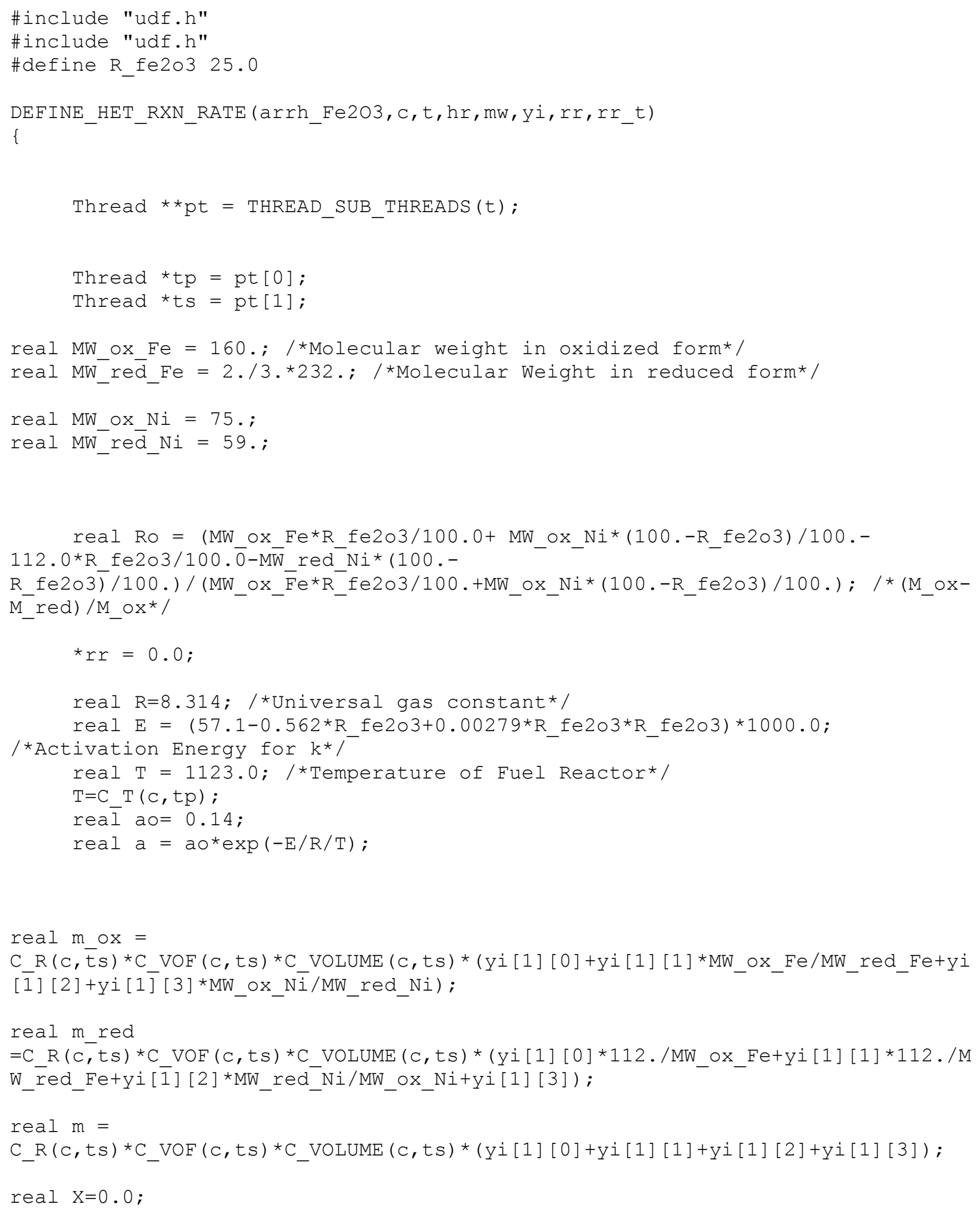




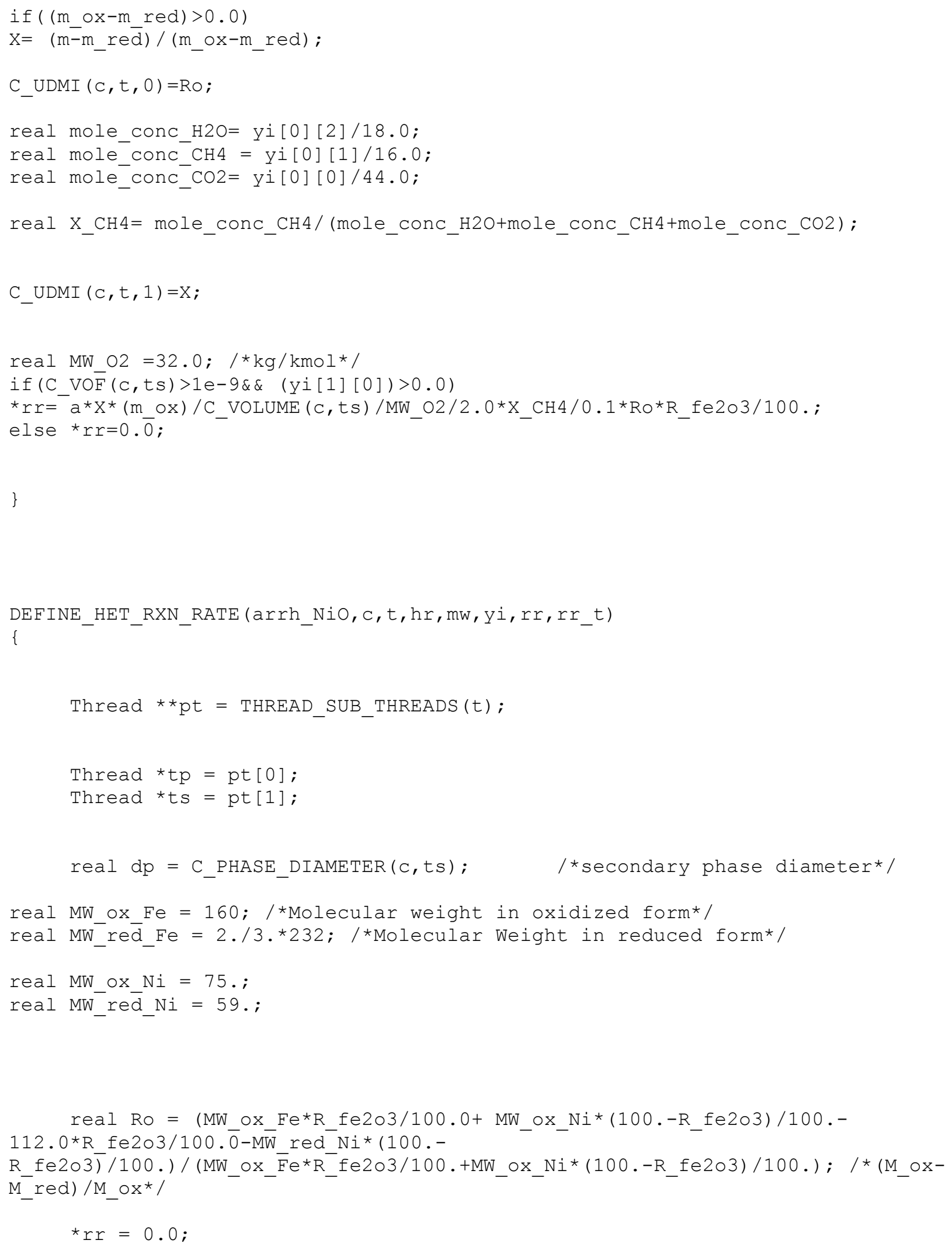




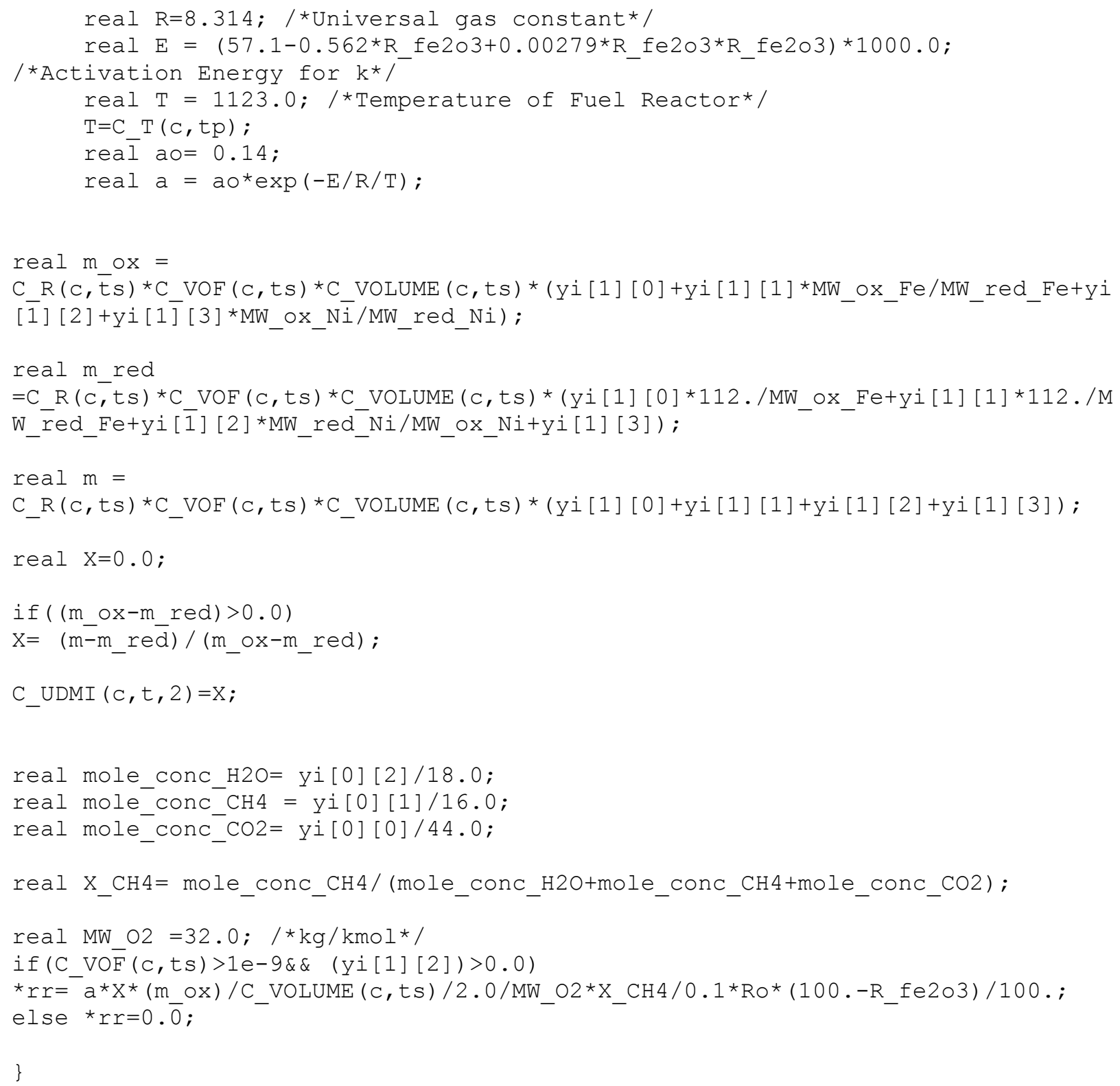




\section{APPENDIX 4: User Defined Functions for Langroundi et al. Viscoplastic Model}

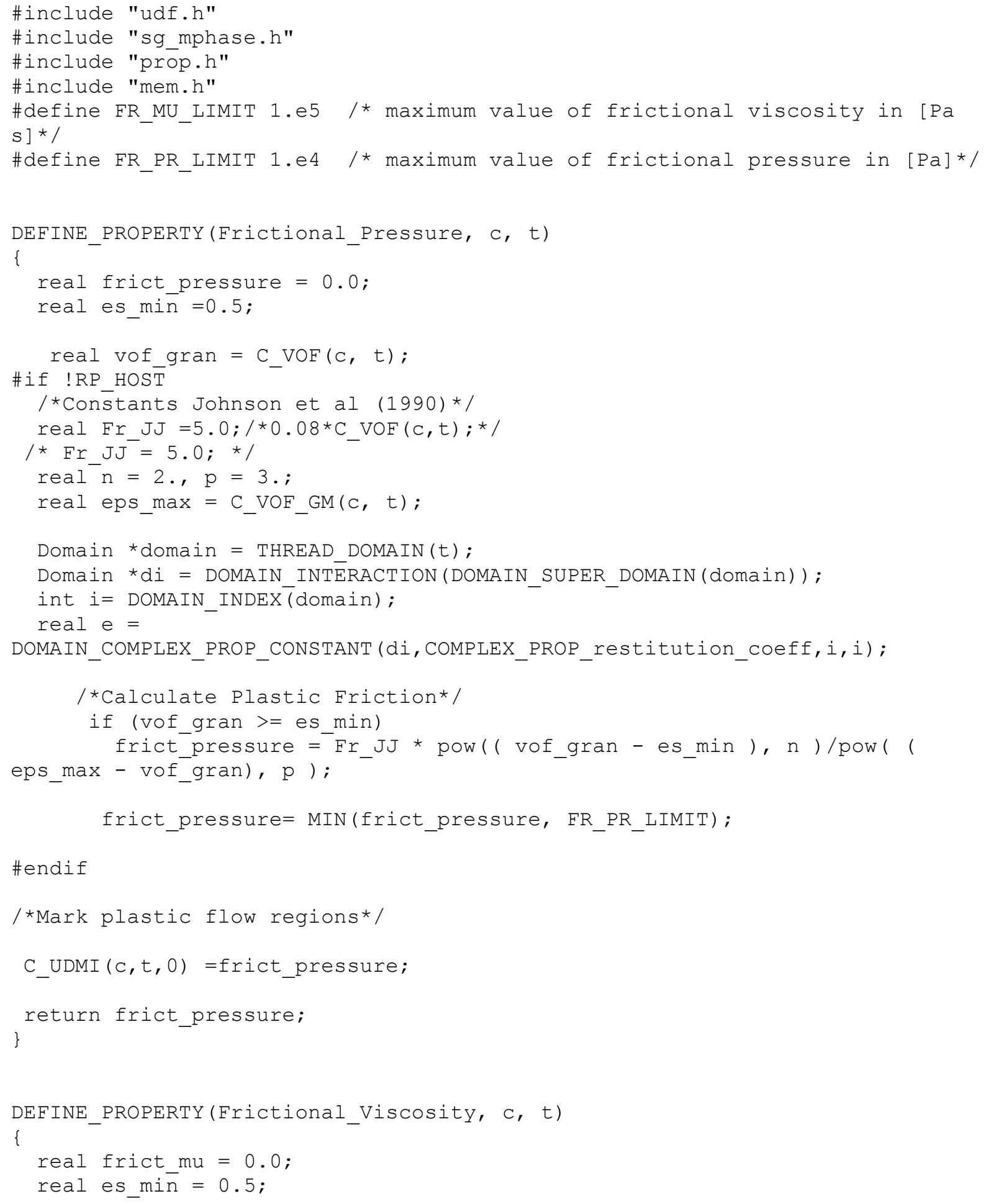




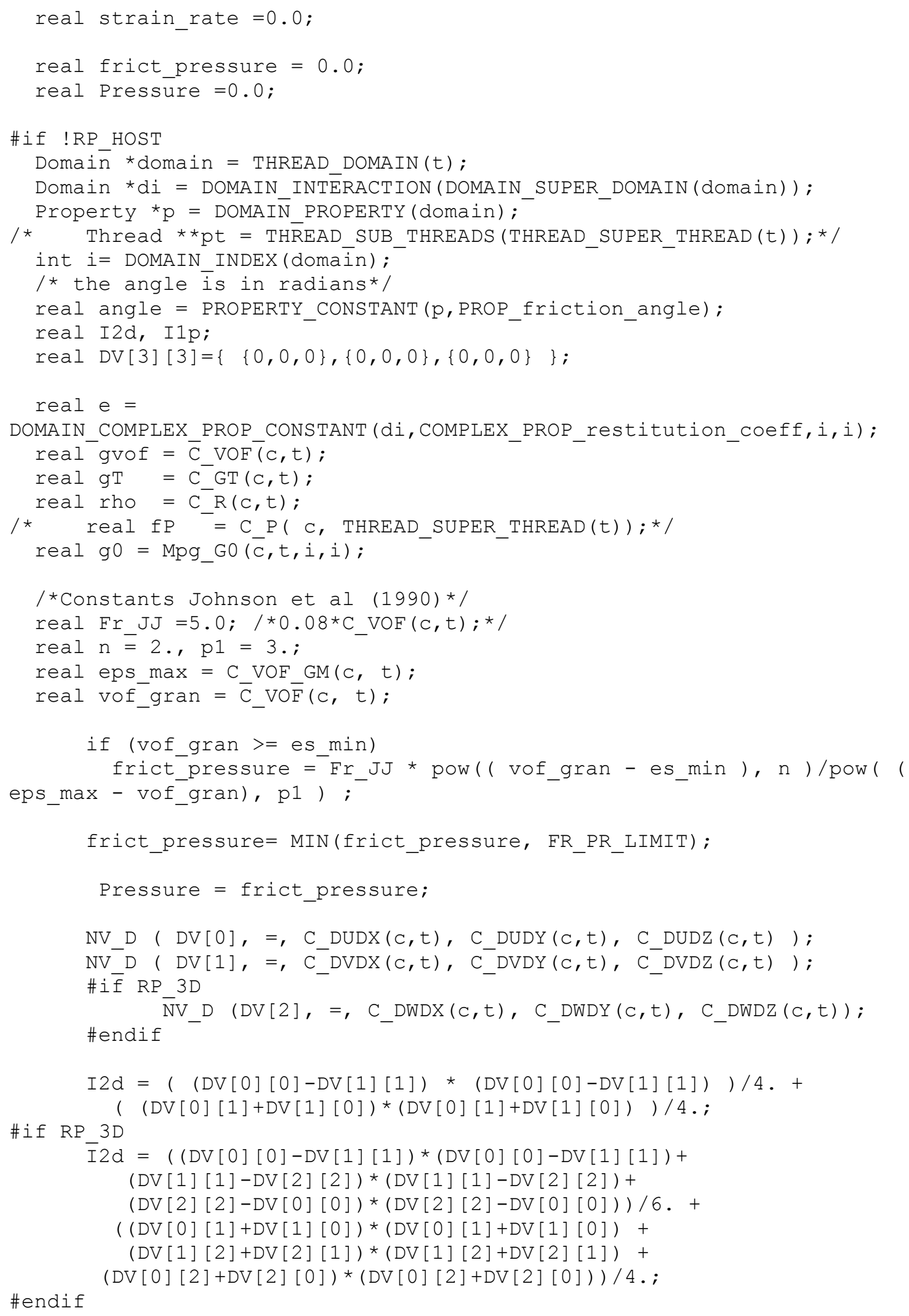




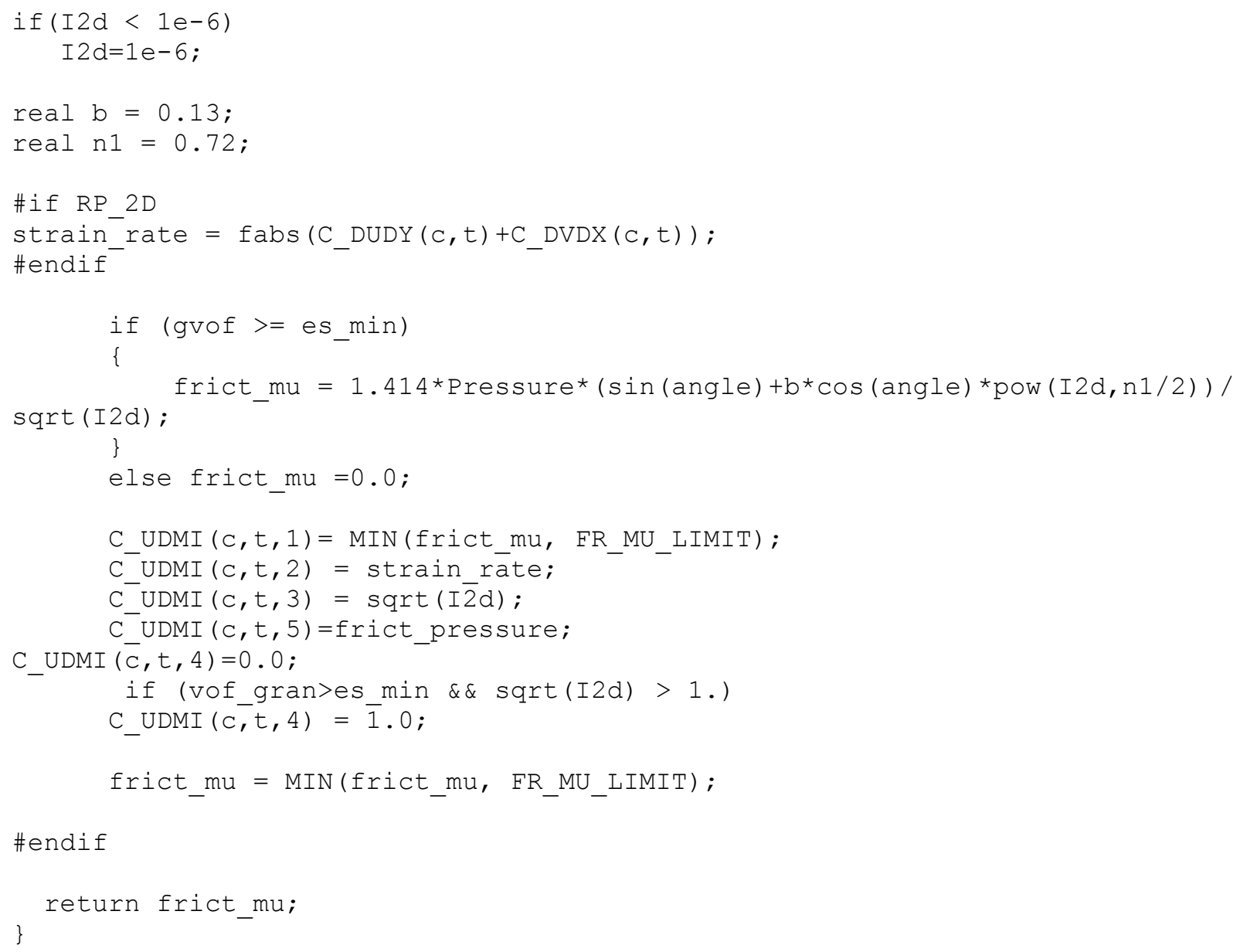




\section{APPENDIX 5: User Defined Drag Law}

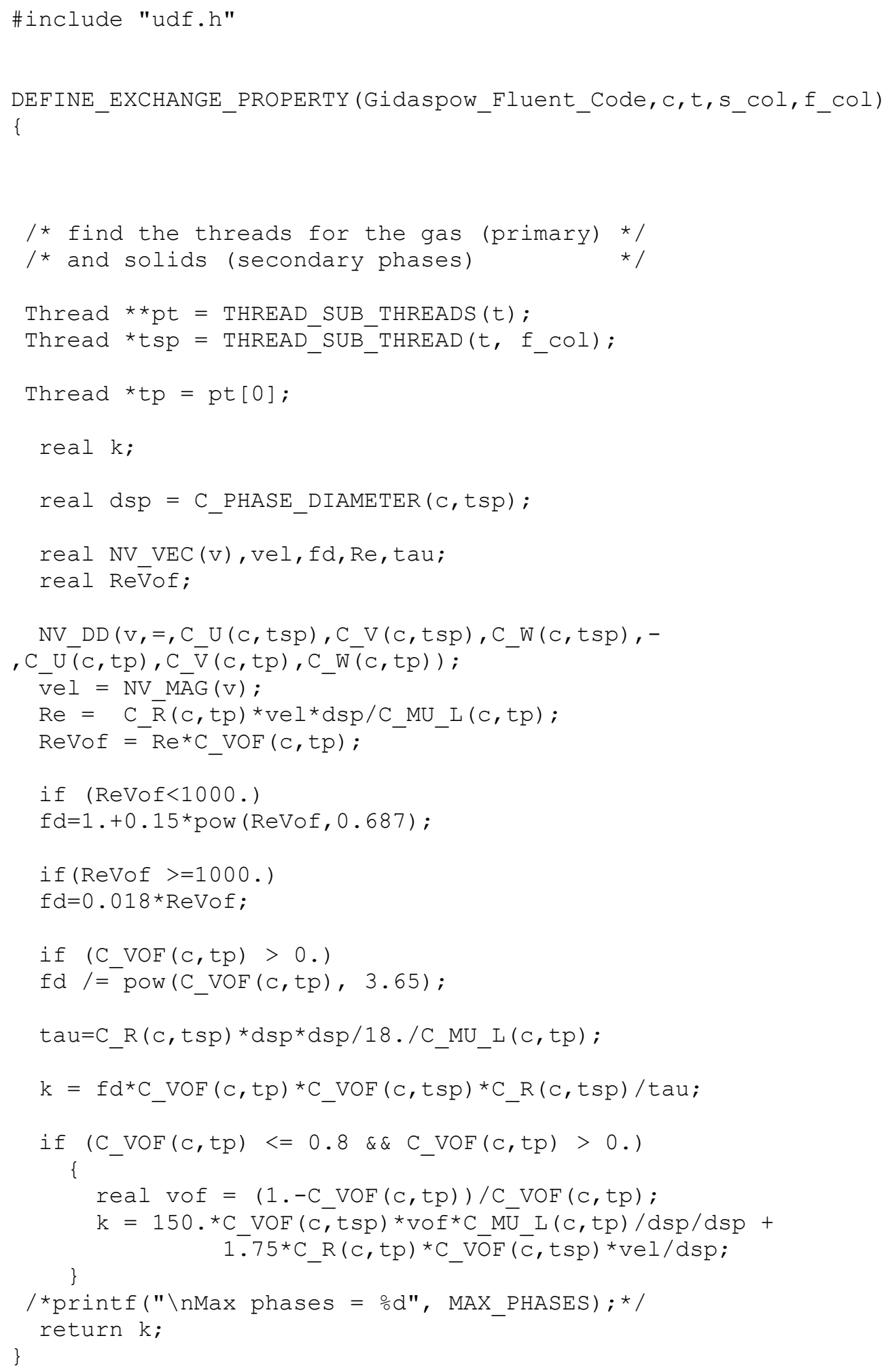

188

John H. Virginia University Libraries, ou=Acquisitions Department, Hagen $\quad \begin{aligned} & \text { iemail=John. Hagen@mail.wvu.edu } \\ & \text { c=Us } \\ & \text { Date: 2010.12.14 12:18:40-05'00' }\end{aligned}$ 